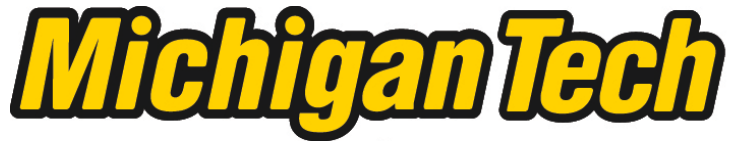 \\ Michigan Technological University Create the Future Digital Commons @ Michigan Tech
}

Dissertations, Master's Theses and Master's Reports - Open

Dissertations, Master's Theses and Master's

Reports

2003

Reynolds-stress turbulence model in the KIVA code for engine simulation

Yeow-Khern Siow

Michigan Technological University

Follow this and additional works at: https://digitalcommons.mtu.edu/etds

Part of the Mechanical Engineering Commons

Copyright 2003 Yeow-Khern Siow

\section{Recommended Citation}

Siow, Yeow-Khern, "Reynolds-stress turbulence model in the KIVA code for engine simulation", Dissertation, Michigan Technological University, 2003.

https://doi.org/10.37099/mtu.dc.etds/408

Follow this and additional works at: https://digitalcommons.mtu.edu/etds

Part of the Mechanical Engineering Commons 


\title{
A REYNOLDS-STRESS TURBULENCE MODEL IN THE KIVA CODE FOR ENGINE SIMULATION
}

\author{
by \\ YEOW-KHERN SIOW
}

\begin{abstract}
A DISSERTATION
Submitted in partial fulfillment of the requirements

for the degree of

DOCTOR OF PHILOSOPHY

(Mechanical Engineering-Engineering Mechanics)
\end{abstract}

MICHIGAN TECHNOLOGICAL UNIVERSITY

2003 
This dissertation titled "A Reynolds-Stress Turbulence Model in the KIVA Code for Engine Simulation" is hereby approved in partial fulfillment of the requirements for the degree of DOCTOR OF PHILOSOPHY in the field of MECHANICAL ENGINEERINGENGINEERING MECHANICS.

\author{
Department: Mechanical Engineering and \\ Engineering Mechanics
}

Dissertation Advisor:

Dr. Song-Lin Yang Date

Department Chair:

Dr. William Predabon

Date 


\title{
A REYNOLDS-STRESS TURBULENCE MODEL IN THE KIVA CODE FOR ENGINE SIMULATION
}

\author{
Yeow-Khern Siow \\ Department of Mechanical Engineering-Engineering Mechanics \\ Michigan Technological University
}

\begin{abstract}
A Reynolds-Stress Turbulence Model has been incorporated with success into the KIVA code, a computational fluid dynamics hydrocode for three-dimensional simulation of fluid flow in engines. The newly implemented Reynolds-stress turbulence model greatly improves the robustness of KIVA, which in its original version has only eddy-viscosity turbulence models. Validation of the Reynolds-stress turbulence model is accomplished by conducting pipe-flow and channel-flow simulations, and comparing the computed results with experimental and direct numerical simulation data. Flows in engines of various geometry and operating conditions are calculated using the model, to study the complex flow fields as well as confirm the model's validity. Results show that the Reynolds-stress turbulence model is able to resolve flow details such as swirl and recirculation bubbles. The model is proven to be an appropriate choice for engine simulations, with consistency and robustness, while requiring relatively low computational effort.
\end{abstract}




\section{ACKNOWLEDGEMENTS}

I would like to thank my academic advisor, Professor Song-Lin Yang, for his guidance throughout the years. The knowledge that he has so selflessly imparted in me is invaluable and will be cherished. The advice that he has given, and experience shared, will always reside in me. His trust and care have helped me grow as a researcher, and will forever be appreciated.

I would also like to thank Professor Oner Arici, Professor Scott Post, and Professor Franz Tanner for serving in my defense committee. Their comments and suggestions have been of great value and importance.

This research was funded by NASA Glenn Research Center, and I am grateful for the technical assistance, experimental data, and valuable input provided by our NASA collaborators, including Dr. Paul Penko, Mr. Robert Tacina, Dr. Dan Bulzan, Dr. John Marek, Dr. Krishna Kundu, and Mr. Anthony Iannetti.

Last but certainly not least, to all my friends and family, thank you. Life would be meaningless without you. 


\section{TABLE OF CONTENTS}

List of tables

vii

1. Introduction 1

1.1. Background 1

1.2. Study of Turbulence 2

1.3. Engine Applications 4

1.4. Research Objectives 5

1.5. Dissertation Outline 5

2. Numerical Studies of Turbulence $\quad 7$

2.1. Governing Equations of Fluid Flow 8

2.2. Direct Numerical Simulation (DNS) 10

2.3. Large-Eddy Simulation (LES) 11

2.4. Reynolds-Averaged Navier-Stokes 11

2.4.1. Turbulent-Viscosity Modeling 13

2.4.2. Reynolds-Stress Modeling 15

3. Reynolds-Stress Turbulence Modeling Equations 16

3.1. Modeling of Diffusion 18

3.2. Modeling of Dissipation 19

3.3. Modeling of Pressure-Rate-of-Strain 19

3.4. Dissipation-Rate Transport Equation 22

3.5. Modeling Constants 23

3.6. Wall Effects 24

4. Implementation of RSTM in the KIVA Code 27

4.1. The KIVA Code 28

4.1.1. The Governing Equations 28

4.1.2. The Numerical Scheme 29

4.1.3. Solution Procedure in Phase B 38

4.2. Discretization of Reynolds-Stress Transport Equations 39

4.3. Discretization of Dissipation-Rate Equation 42

4.4. RSTM Solution Procedure 47

4.5. Validations 52

4.5.1. Pipe Flow $\quad 52$

4.5.2. Channel Flow 54

5. Applications 57

5.1. Lean-Direct-Injection (LDI) Combustor with Axial Swirlers 58

$\begin{array}{ll}\text { 5.1.1. Introduction } & 58\end{array}$

5.1.2. RSTM and Code Implementation 61 
5.1.3. Description of the Problem 61

5.1.4. Results and Discussions 63

5.1.5. Conclusion $\quad 69$

5.2. Lean-Premixed-Prevaporized Combustor 83

5.2.1. Introduction $\quad 83$

5.2.2. The CFD Code 85

5.2.3. The Kinetic Mechanism 86

5.2.4. Experimental Configuration 87

5.2.5. Results $\quad 89$

5.2.6. Discussion of Results 90

5.2.7. Computer Execution $\quad 92$

5.2.8. Conclusion 93

5.3. LDI Combustor with Discrete-Jet Swirlers 100

5.3.1. Introduction 100

5.3.2. The National Combustion Code 103

5.3.3. The KIVA-3V Code with RSTM 103

5.3.4. Problem Description and Computational Mesh 104

5.3.5. Discrete-Jet Swirler Array Flow Physics 105

5.3.6. Flow Conditions and LDV Measurements 106

5.3.7. Results and Discussion 107

5.3.8. Conclusion 112

5.4. Engine Flow Simulation 121

$\begin{array}{ll}\text { 5.4.1. Introduction } & 121\end{array}$

5.4.2. Results and Discussion $\quad 123$

5.4.3. Conclusion 135

5.5. LDI Combustor with Radial Swirlers 150

5.5.1. Results and Discussion $\quad 150$

5.6. Design of Radial Air Swirler Blade Lengths 162

5.6.1. Introduction 162

5.6.2. Swirl Number 164

5.6.3. Computational Domain and Boundary Conditions 165

5.6.4. Results and Discussion 166

5.6.5. Conclusion 168

6. Other Contributions 173

6.1. Total-Variation Diminishing Scheme for the Convective Transport 173

6.1.1. Implementing TVD in KIVA 174

6.2. Reduced Kinetic Mechanism 180

6.2.1. Implementation in KIVA 180

$\begin{array}{lll}\text { 7. Conclusions } & 182\end{array}$

$\begin{array}{ll}\text { References } & 185\end{array}$

Appendix A A-1

Appendix B $\quad$ B-1

Appendix C $\quad$ C-1 


\section{LIST OF TABLES}

Table 3.1: $\quad$ RSTM Model Constants 23

Table 5.1: $\quad$ Inlet Turbulence Values $\quad 82$

Table 5.2: $\quad$ Geometry and Flow Parameters $\quad 88$

Table 5.3: $\quad$ 18-Hole Plate, $25 \mathrm{~cm}$ Measurement Station $\quad 89$

Table 5.4: $\quad$ Machine Description $\quad 92$

$\begin{array}{lll}\text { Table 5.5: } \quad \text { Grid Density } & 105\end{array}$

Table 5.6: $\quad$ Blade Length Cases 165

Table 5.7: $\quad$ CTRZ Sizes 166 


\section{Chapter 1}

\section{Introduction}

\subsection{Background}

Numerical study of turbulence in engines requires high-level modeling technique to resolve the detail of the flow. Presently, the most popular turbulence models lack the capability to describe turbulence structures without the need for ad hoc modifications. The performance of these models depends highly on the type and geometry of the flow, and the model coefficients often need adjustments on a case-by-case basis. In addition, due to the inherent nature of these models, certain turbulence characteristics are unable to be resolved.

In order to study and predict turbulence behavior in more detail and completeness, use of higher-order models is necessary. For engine flow simulation, in particular, a robust 
turbulence model is especially desirable. The combustion chamber inside an engine typically has a complex geometry, multiple inflow and outflow openings, and/or other structural features. The flow field is therefore highly turbulent, and the choice of an appropriate turbulence model is of primary concern.

The performance of a turbulence model is judged not only by its accuracy, but also its efficiency and the computational effort it requires on a given machine platform. A number of advanced turbulence models and simulation technique require tremendous computational resources and time, thus having limited applicability. To achieve higher practicality for current-generation machines, the turbulence model should be relatively inexpensive.

\subsection{Study of Turbulence}

Nearly all fluid flows are turbulent. The ability to understand and predict turbulence is important in many engineering applications, such as the wings of an airplane and the engine of a vehicle. Yet, understanding turbulence is perhaps the most challenging aspect of fluid mechanics. It is still considered an "unsolved mystery" in fluid physics.

The study of turbulence includes experiments and theoretical modeling. Experiments are observations and measurements of fluid properties using specialized instruments such as a laser-Doppler velocimetry (LDV) system. However, experiments can be costly and time-consuming to set up and perform. Another approach to study turbulence is to devise mathematical formulations that model, and predict, turbulence behavior. Many turbulence theories exist in the literature, and modeling of turbulence are based on these theories. 
Turbulence theories and modeling techniques have been an important subject in fluid mechanics and engineering. A vast majority of these techniques contain a number of equations whose solution needs to be obtained numerically. The computational study of turbulence, therefore, is an essential aspect of computational fluid dynamics, or CFD - a useful and cost-effective tool for scientists and engineers.

Recent advancement in computing power has enabled the use of direct numerical simulation, or DNS, to study low Reynolds number flow involving simple geometry. This method, despite its limited applicability, has provided valuable data - in particular, turbulence quantities in the near-wall region - for validating the accuracy of turbulence models by comparing the computed results. It is, however, extremely expensive to compute.

As an alternative to avoid the computational overhead required by DNS, many researchers have sought large-eddy simulation, or LES, for accuracy and computing performance. Despite its wider applicability than DNS in simulating higher Reynoldsnumber flows, however, LES has not reached the computational efficiency for everydaycalculations of engineering flows on low-cost machines. It still requires a tremendous amount of CPU time and memory, and reasonable turnaround time can only be achieved by the use of costly supercomputers or a large network or cluster.

The next level of computational study of turbulence involves the Reynoldsaveraged Navier-Stokes (RANS) approach, under which two modeling techniques exist. The first uses the turbulent-viscosity hypothesis, on which a number of popular models have based. In particular, the two-equation models are widely used in most commercial CFD codes. In spite of its popularity, all models based on the turbulent-viscosity 
hypothesis suffer from many drawbacks that are well-documented in the literature. The most popular turbulence models today are the eddy-viscosity models, sometimes referred to as two-equation models. Despite their popularity and ease of implementation, ad hoc modifications to the model coefficients are often necessary to suit the needs of the particular types of flow. Furthermore, these models are generally unable to accurately predict complex fluid flows due to the inherent nature of the eddy-viscosity approximation used in the formulations.

The second technique under the RANS approach involves solving the transport equations for the Reynolds-stresses, which stem from the Reynolds-averaging procedure carried out on the Navier-Stokes equation. This modeling technique provides a reasonable balance between physical accuracy and computational cost, and will be explored in detail in this dissertation.

\subsection{Engine Applications}

Fluid flows in engines of any type, e.g., internal combustion engines or gas turbine combustors, are always turbulent. In engine design, turbulence is an important and primary consideration, and is often intentionally induced by means of geometry, such as a bowl in the piston and a flame-holder inside a combustor. The presence of turbulence can enhance air-fuel mixing and the transport of mass, momentum and energy. However, excessive amount of turbulence may disrupt the flow field and bring about undesirable effects, such as destabilizing flame during the combustion process. Therefore, the understanding and control of turbulence are necessary to achieve efficient combustion, thereby increasing performance and reducing combustion wastes. 
Emissions of pollutants, such as $\mathrm{NO}_{\mathrm{x}}, \mathrm{CO}, \mathrm{CO}_{2}$, unburned hydrocarbons, and particulates (soots), are a key issue concerning the performance of gas turbine combustors and IC engines. In order to achieve clean combustion, it is necessary to be able to predict the behavior of turbulence and its effects on the flow field, chemical kinetics, and/or fuel spray, all of which may be highly coupled. To successfully predict turbulent reactive flow, therefore, it is imperative to successfully predict turbulence in the flow field.

\subsection{Research Objectives}

The objectives of the current research include:

(1) To implement a higher-order, computationally practical turbulence model in a widely available, open source research code, KIVA-3V Release 2;

(2) To validate the model;

(3) To apply the model to various types of engines, primarily gas turbine combustors in jet engines; and

(4) To prove the model's applicability, accuracy, and robustness

\subsection{Dissertation Outline}

In the next chapter, Chapter 2, an overview of the status quo in turbulence modeling is given. In Chapter 3, the equations for Reynolds-stress turbulence model (RSTM) are explained in detail. In Chapter 4, a description of KIVA, its numerics and structure, the implementation of RSTM in KIVA, and its validation are presented. The applications of RSTM in engines of various geometry are included in Chapter 5, where the

computed results are compared with available experimental data. Chapter 6 presents 
additional completed work for the improvements of KIVA, and finally, research summary, conclusions, contributions and significant findings are given in Chapter 7.

Code-related detail and a chemical kinetic mechanism are included in the appendices. 


\section{Chapter 2}

\section{Numerical Studies of Turbulence}

A tremendous amount of research in fluid mechanics has been devoted to understanding the physics of turbulence. Due to the complex nature of turbulence and the equations that describe it, many researchers have relied on computational techniques to seek solution - either directly simulating the time- and space-evolution of turbulence, or modeling the phenomenon by using one or more modeled equations.

All these simulation/modeling methods encompass several levels of accuracy, complexity, and computational efforts. The fundamental principles behind these techniques differ significantly, from direct solution of all scales in the Navier-Stokes equation to modeling the global turbulence behavior using an algebraic equation. In this 
chapter, each of these methods will be briefly introduced, with emphasis on the approach utilizing the Reynolds-averaging technique.

While direct numerical simulation (DNS) represents the highest level of technique available in the computations of turbulence, modeling based on the Reynolds-averaged Navier-Stokes (RANS) equations represents another genre of approaches employing statistics. Each method has its own merits and limitations, and will be discussed in this chapter.

In order to fully appreciate the simulation and modeling techniques to be presented, it is appropriate to first present the equations that govern fluid motions, known as Navier-Stokes equations. It is described next.

\subsection{Governing Equations of Fluid Flow}

In this section, the governing equations of fluid flow, namely the continuity and momentum equations, will be presented. The primary subject of this dissertation is concerned with mean-flow quantities (to be introduced in Section 2.4 below), and the effects of turbulence in the mean-flow equations are most direct in the momentum equations. On the other hand, turbulence influence on the fluid internal energy, such as the molecular diffusion and turbulent transport terms, is usually small for low-Mach number flows, and may be neglected in the energy equation [Wilcox, 1993]. Therefore, only the continuity and momentum equations will be discussed here.

In all subsequent mathematical expressions, symbols in bold font or having a single-index subscript represent vectors quantities, while variables with a double-index subscript are second-order tensors. Both vector and Cartesian-tensor notations will be 
used alternately in the following chapters. Tensor notation is particularly handy when compactness is needed.

All engineering material flows must satisfy the law of mass conservation. When applied to an infinitesimal control volume, the result is the differential continuity equation,

$$
\frac{\partial \rho}{\partial t}+\nabla \cdot(\rho \overparen{U})=0
$$

where $\rho$ is the density and $\overparen{U}$ is the instantaneous velocity of the fluid.

The momentum equation, derived from Newton's second law, relates the acceleration of fluid particles to the forces they experience. In conservative differential form, this equation is

$$
\frac{\partial}{\partial t}(\rho \overparen{\boldsymbol{U}})+\nabla \bullet(\rho \overparen{\boldsymbol{U}} \overparen{\boldsymbol{U}})=-\nabla \widehat{p}+\rho \boldsymbol{g}+\nabla \bullet \widehat{\tau}_{i j}
$$

where $\widehat{p}$ is the instantaneous fluid pressure, $\boldsymbol{g}$ the gravitational force per unit mass, and $\widehat{\tau}_{i j}$ the stress tensor, defined as

$$
\widehat{\tau}_{i j}=\mu\left(\frac{\partial \widehat{U}_{i}}{\partial x_{j}}+\frac{\partial \overparen{U}_{j}}{\partial x_{i}}-\frac{2}{3}{\frac{\partial \overparen{U}^{k}}{\partial x_{k}}} \delta_{i j}\right)
$$

The quantity $\mu$ is the molecular viscosity of the fluid. Eq.(2.2) is also known as the Navier-Stokes equations, first conceived by applied mathematician/engineer Claude-Louis Navier in 1821 . The same equations were later deduced by mathematician George Stokes in 1845 , based on theories of fluid friction.

Navier-Stokes equations are nonlinear partial differential equations, and in Cartesian coordinate system have three components, one in each principal direction. The equations may appear simple, but they contain complete governance of fluid motions of all 
scales in time and length. Therefore, numerically solving for Navier-Stokes equations can provide the greatest details in the flow. However, due to the tremendous amount of information carried in the equations, seeking direct solution requires extremely large amount of computing resources and power.

To more practically obtain solutions from Navier-Stokes equations, mathematical modeling employing statistical tools are necessary. Several modeling techniques exist in the literature, and are of great interest to the fluid research community.

All these methods will be discussed next.

\subsection{Direct Numerical Simulation (DNS)}

Until the 1970s, calculations using DNS had been inconceivable due to insufficient computing power [Pope, 2000]. The advancement in computer hardware and software technology, the constant increase in processor speed and memory, as well as the development of parallel architecture has allowed DNS to be employed for turbulent fluid flows.

DNS seeks solutions to the Navier-Stokes equation, resolving all time and length scales of turbulence. It is fundamentally the simplest method to implement, since no modeling is required. The accuracy the solution can achieve is unparalleled among all techniques. However, it is extremely expensive to simulate even the simplest types of flow (e.g., homogeneous turbulence), primarily due to the super-fine grid required to resolve the small-scale turbulence structures, as well as the small time-steps for the time-scales of the smallest eddies. Therefore, DNS is largely restricted to low- to moderate-Reynoldsnumber flows and simple geometries. 


\subsection{Large-Eddy Simulation (LES)}

A majority of LES applications is in geophysics, such as oceanography and the study of atmospheric boundary layers, examples of which can be found in the references in Galperin and Orszag [1993]. For most LES studies, the size of the problem is often very large as well as three dimensional, and the difference in length scale is significant, e.g., the fine grid near the earth's crust. The result is an enormous amount of grid points in the flow domain. Therefore, obtaining a solution in a reasonable time frame requires an efficient method that is less computationally expensive than DNS, while providing accuracy comparable to DNS. This motivation has helped propelled the development and popularity of large-eddy simulation.

In LES, the velocity field is filtered using a low-pass filter. The filtered field, representing the large-scale turbulent motion, is resolved directly, while the unfiltered (or "subgrid") velocity is modeled, typically employing the eddy-viscosity hypothesis. Consequently, unlike DNS where most of the computing overhead are consumed on the resolution of small-scale, dissipative motion, LES is considerably less expensive since only the large eddies are directly simulated.

Details on the LES concept, methodology and further applications can be found in the literature, such as Reynolds [1980], Galperin and Orszag [1993], and Pope [2000].

\subsection{Reynolds-Averaged Navier-Stokes}

In the Reynolds-averaged Navier-Stokes (RANS) approach, instead of directly solving for the turbulent field, solution is obtained by solving the mean-velocity field. In 
RANS, the velocity field is decomposed into the mean-field and random (or fluctuating) parts, i.e.,

$$
\overparen{U}(\boldsymbol{x}, t)=\boldsymbol{U}(\boldsymbol{x}, t)+\boldsymbol{u}(\boldsymbol{x}, t)
$$

where $\widehat{\boldsymbol{U}}(\boldsymbol{x}, t)$ represents the instantaneous velocity field, $\boldsymbol{U}(\boldsymbol{x}, t)$ the mean-velocity field, and $\boldsymbol{u}(\boldsymbol{x}, t)$ the fluctuating part. The relation in Eq. (2.4) is also known as Reynolds decomposition.

It should be noted that, in taking the mean of velocity field, density-weighted averaging (or Favre averaging) is used to account for compressibility of the flow. For all subsequent equations, all vector and tensor variables (e.g., velocity $\boldsymbol{U}$ and the Reynolds stresses, $-\rho u_{i} u_{j}$ ) that occur in the mean-flow and model equations are understood as Favre-averaged quantities.

Accordingly, substituting the above relation into the (compressible) continuity equation,

$$
\frac{\partial \rho}{\partial t}+\nabla \bullet(\rho \boldsymbol{U})=0
$$

and, subsequently,

$$
\frac{\partial \rho}{\partial t}+\nabla \bullet(\rho \boldsymbol{u})=0
$$

Taking the mean of Navier-Stokes equation is less straightforward due to the presence of the nonlinear convective term. The final form of the mean-field momentum equation (also known as Reynolds equation), in tensor notation, is

$$
\frac{\partial}{\partial t}\left(\rho U_{i}\right)+\frac{\partial}{\partial x_{j}}\left(\rho U_{i} U_{j}\right)=-\frac{\partial p}{\partial x_{i}}-\frac{\partial}{\partial x_{j}}\left(\rho u_{i} u_{j}\right)+\frac{\partial \tau_{i j}}{\partial x_{j}}
$$


where

$$
\tau_{i j}=\mu\left(\frac{\partial U_{i}}{\partial x_{j}}+\frac{\partial U_{j}}{\partial x_{i}}-\frac{2}{3} \frac{\partial U_{k}}{\partial x_{k}} \delta_{i j}\right)
$$

This Reynolds equation is similar to the Navier-Stokes equation, Eq.(2.2), except

for the term $\frac{\partial}{\partial x_{j}}\left(\rho u_{i} u_{j}\right)$, which has significant contribution to the fluid momentum. Under the RANS approach, there are four equations, i.e., the continuity equation and the vector momentum equation (three components). However, there are more than four unknowns. In addition to the three components of velocity and pressure, there are also Reynolds stresses, $-\rho u_{i} u_{j}$. This presents a closure problem: additional equation(s) must be devised to close the solution, by modeling the Reynolds stresses. In the literature, two basic methods exist for closure, namely turbulent-viscosity modeling and Reynolds-stress modeling.

\subsubsection{Turbulent-Viscosity Modeling}

The turbulent-viscosity modeling, sometimes called eddy-viscosity modeling (EVM), is based on the turbulent-viscosity hypothesis (or Boussinesque hypothesis), in which the deviatoric Reynolds stress is assumed to be proportional to the mean rate of strain, i.e.,

$$
-\rho u_{i} u_{j}+\frac{2}{3} \rho k \delta_{i j}=\mu_{T}\left(\frac{\partial U_{i}}{\partial x_{j}}+\frac{\partial U_{j}}{\partial x_{i}}\right)
$$

where $\mu_{T}$ is called the turbulent viscosity (or eddy viscosity), and $k$ is the turbulent kinetic energy, defined as

$$
k=\frac{1}{2}\left(u_{i} u_{i}\right)=\frac{1}{2}\left(u_{1}^{2}+u_{2}^{2}+u_{3}^{2}\right)
$$


Accordingly, the "effective viscosity," $\mu_{\text {eff }}$, is defined as the sum of molecular viscosity and eddy viscosity, i.e.,

$$
\mu_{e f f}(x, t)=\mu+\mu_{T}(x, t)
$$

Substituting the above relations into the mean-flow momentum equation (or simply, Reynolds equation) gives

$$
\frac{d}{d t}\left(\rho U_{j}\right)=\frac{\partial}{\partial x_{i}}\left[\mu_{e f f}\left(\frac{\partial U_{i}}{\partial x_{j}}+\frac{\partial U_{j}}{\partial x_{i}}\right)\right]-\frac{\partial}{\partial x_{j}}\left(p+\frac{2}{3} \rho k\right)
$$

where $p+\frac{2}{3} \rho k$ is the modified mean pressure, consisting of the thermodynamic pressure $p$ and the turbulent pressure $\frac{2}{3} \rho k$.

For closure, therefore, only $\mu_{T}(x, t)$ needs to be modeled. Several modeling techniques, namely, the zero-equation, one-equation, and two-equation models, are briefly discussed next.

(1). Zero-equation, or algebraic modeling. Here, $\mu_{T}$ is modeled algebraically. For details of this method, please refer to texts such as Pope [2000] and Wilcox [1993].

(2). One-equation modeling. The $\mu_{T}$ is related to the turbulent kinetic energy, $k$, by the equation

$$
\mu_{T}=C \rho k^{1 / 2} \mathbf{l}_{m}
$$

where $C$ is a model constant, and $\mathrm{t}_{m}$ is the mixing lengthscale, which must be specified. The values of $k$ needs to be solved by a single transport equation. The exact equation for the evolution of $k$, for incompressible flow, is [Pope, 2000] 


$$
\frac{D k}{D t}=-\wp+\varepsilon-\frac{\partial}{\partial x_{j}}\left(\frac{p u_{j}}{\rho}+\frac{1}{2} u_{i} u_{i} u_{j}-v \frac{\partial k}{\partial x_{j}}\right)
$$

where

$$
\wp=u_{i} u_{k} \frac{\partial U_{i}}{\partial x_{k}}
$$

which is the production of turbulent kinetic energy (or production), and

$$
\varepsilon=-v \frac{\partial u_{i} \partial u_{i}}{\partial x_{k} \partial x_{k}}
$$

dissipation rate of turbulent kinetic energy, or simply, dissipation.

(3). Two-equation modeling. Under this modeling class, two transport equations are solved for $k$ and $\varepsilon$ in the $k$ - $\varepsilon$ model [Launder and Spalding, 1972], or $k$ and $\omega$ $(=\varepsilon / k)$ in the $k-\omega$ model [Wilcox, 1993].

This approach is relatively easy to implement, has low computational overhead while providing reasonable accuracy. Due to the nature of Boussinesque hypothesis, however, the eddy-viscosity models are unable to account for flow separation, swirling flow, secondary motion, curvature, or any large transport effects [Launder, 1989; Yang et al., 2000].

\subsubsection{Reynolds-Stress Modeling}

The other modeling method under the RANS approach is the Reynolds-stress turbulence modeling, sometimes called the second-moment closure modeling. A description of the model detail is given in the next chapter. 


\section{Chapter 3}

\section{Reynolds-Stress Turbulence Modeling Equations}

Reynolds-stress turbulence models, or RSTMs, represent the highest level of turbulence modeling within the framework of the Reynolds-averaged Navier-Stokes approach. It provides the most natural and logical way for the closure of the RANS equations [Hanjalic, 1997]. It is therefore sometimes also referred to as the secondmoment closure model (SCM), or Reynolds-stress closure model (RSCM). Reynoldsstress turbulence models stands between large-eddy simulation and two-equation turbulent-viscosity models in terms of physical correctness as well as computational overhead. 
By taking the ensemble average of the product of $u_{j}$ with the momentum equation for the fluctuating velocity, one can obtain the exact transport equation for the Reynolds stresses:

$$
\frac{\partial}{\partial t}\left(\rho u_{i} u_{j}\right)+\frac{\partial}{\partial x_{k}}\left(\rho U_{k} u_{i} u_{j}\right)=P_{i j}+D_{i j}+\varepsilon_{i j}+\Phi_{i j}
$$

where $u_{i} u_{j}$ is a Favre-averaged (density-weighted) quantity. The tensor terms on the righthand side are, in exact form, the

1. production,

$$
P_{i j}=-\left(\rho u_{i} u_{k} \frac{\partial U_{j}}{\partial x_{k}}+\rho u_{j} u_{k} \frac{\partial U_{i}}{\partial x_{k}}\right)
$$

2. diffusion (viscous, triple-velocity correlation, and pressure),

$$
D_{i j}=\frac{\partial}{\partial x_{k}}\left(\mu \frac{\partial}{\partial x_{k}}\left(u_{i} u_{j}\right)-\rho\left(u_{i} u_{j} u_{k}\right)-p\left(u_{i} \delta_{j k}+u_{j} \delta_{i k}\right)\right)
$$

where

$$
\begin{aligned}
& D_{i j}^{v}=\frac{\partial}{\partial x_{k}}\left(\mu \frac{\partial}{\partial x_{k}}\left(u_{i} u_{j}\right)\right)=\text { viscous diffusion, } \\
& D_{i j}^{t v}=\frac{\partial}{\partial x_{k}}\left(-\rho\left(u_{i} u_{j} u_{k}\right)\right)=\text { triple-velocity correlation, and } \\
& D_{i j}^{p}=\frac{\partial}{\partial x_{k}}\left(-p\left(u_{i} \delta_{j k}+u_{j} \delta_{i k}\right)\right)=\text { pressure diffusion }
\end{aligned}
$$

3. dissipation,

$$
\varepsilon_{i j}=-2 \mu\left(\frac{\partial u_{i} \partial u_{j}}{\partial x_{k} \partial x_{k}}\right) \text { and }
$$

4. pressure-strain redistribution (or pressure-rate-of-strain), $\Phi_{i j}$, 


$$
\Phi_{i j}=p\left(\frac{\partial u_{i}}{\partial x_{j}}+\frac{\partial u_{j}}{\partial x_{i}}\right)
$$

Clearly, the diffusion (except the viscous diffusion of Reynolds stresses), dissipation, and the pressure-strain redistribution terms, Eqs. (3.5-3.8), need to be modeled.

\subsection{Modeling of Diffusion}

A number of models for the diffusion are available in the literature [e.g., Shir, 1973; Daly and Harlow, 1970; Hanjalic and Launder, 1972; Lumley, 1978], with the

model proposed by Daly and Harlow being the most conventionally-adopted, based on their generalized gradient diffusion hypothesis (GGDH):

$$
D_{i j}=C_{s} \frac{\partial}{\partial x_{k}}\left(\frac{k}{\varepsilon} u_{k} u_{l} \frac{\partial}{\partial x_{l}}\left(u_{i} u_{j}\right)\right)
$$

where $C_{s}=0.22$.

These models, including Eq. (3.9), vary in complexity and accuracy. However, in most types of flow, the effect of the diffusion process is usually much smaller than the other source (or sink) terms [Launder, 1989]. Diffusion contributes slightly to the Reynolds stress budget, since it tends to spatially redistribute the Reynolds stresses, instead of more directly affect the transport process. Furthermore, based on a study conducted by Lien and Leschziner [1994], better numerical stability can be achieved by using a simplified model under the assumption of isotropic stress-diffusion:

$$
D_{i j}=\frac{\partial}{\partial x_{k}}\left(\frac{\mu_{t}}{\sigma_{\mathrm{\kappa}}} \frac{\partial}{\partial x_{k}}\left(u_{i} u_{j}\right)\right)
$$


where $\mu_{t}=\rho C_{\mu} \frac{k^{2}}{\varepsilon}$ and $\sigma_{\mathrm{\kappa}}$ is the Prandtl number for turbulence energy, a modeling constant to be given below.

\subsection{Modeling of Dissipation}

The dissipation rate tensor $\varepsilon_{i j}$, Eq. (3.7), carries significant contributions to the transfer of turbulent energy. For high-Reynolds-number flows, based on Richardson's theory of energy cascade [Richardson, 1922] and Kolmogorov's hypotheses, the smallscale turbulent motions, or eddies, are dissipated by viscous action and, more importantly, statistically isotropic in nature. Therefore, for flows at sufficiently high Reynolds number (e.g., not in the near-wall region for wall-bounded flows), the dissipation $\varepsilon_{i j}$ can be modeled by assuming local isotropy, and usually expressed as

$$
\varepsilon_{i j}=-\frac{2}{3} \rho \varepsilon \delta_{i j}
$$

\subsection{Modeling of Pressure-Rate-of-Strain}

In modeling the RSTMs, many researchers [e.g., Launder et al., 1975; Lumley, 1978; Speziale et al., 1991] have focused on the pressure-strain redistribution. One of the reasons is the fact that this tensor, Eq. (3.8), and the pressure diffusion, Eq. (3.6), together form just one of many possible decompositions of the "velocity-pressure-gradient tensor," $\Pi_{i j}$. i.e., 


$$
\begin{aligned}
\Pi_{i j}=-\left(u_{i} \frac{\partial p}{\partial x_{j}}+u_{j} \frac{\partial p}{\partial x_{i}}\right) & =\Phi_{i j}+D_{i j}^{p} \\
& =p\left(\frac{\partial u_{i}}{\partial x_{j}}+\frac{\partial u_{j}}{\partial x_{i}}\right)+\frac{\partial}{\partial x_{k}}\left(-p\left(u_{i} \delta_{j k}+u_{j} \delta_{i k}\right)\right)
\end{aligned}
$$

Other alternatives for the decomposition of $\Pi_{i j}$ exist, e.g., those by Lumley [1975] and Mansour et al. (1988). In this thesis work Eq. (3.12) is used, since it is regarded as the classical decomposition of $\Pi_{i j}$, as well as for convenience.

The pressure-rate-of-strain, $\Phi_{i j}$, Eq. (3.8), is a traceless tensor, i.e.,

$$
\Phi_{i i}=2 \overline{p \nabla \bullet \boldsymbol{u}}=0
$$

Therefore, it is absent from the turbulent kinetic energy $\left(k=1 / 2\left(u_{i} u_{i}\right)\right)$ equation, but instead, it serves to redistribute energy among the Reynolds stresses, and is of crucial importance [Pope, 2000]. Several possibilities exist in modeling the pressure-strain redistribution. For instance, under the homogeneous turbulence assumption, $\Phi_{i j}$ can be decomposed into two parts:

$$
\Phi_{i j}=\Phi_{i j, 1}+\Phi_{i j, 2}+\Phi_{i j, w}
$$

where $\Phi_{i j, 1}$ is the "slow" or "return-to-isotropy" pressure-strain redistribution. A general nonlinear model for $\Phi_{i j, 1}$ can be written as

$$
\Phi_{i j, 1}=-\rho \varepsilon\left[C_{1} a_{i j}-C_{1 s}\left(a_{i k} a_{k j}-\frac{1}{3} A_{2} \delta_{i j}\right)\right]
$$

where the terms $a_{i j}$ and $A_{2}$ are the normalized anisotropic stress tensor and its first invariant, respectively:

$$
a_{i j}=\frac{u_{i} u_{j}}{k}-\frac{2}{3} \delta_{i j}
$$




$$
A_{2}=a_{i j} a_{j i}
$$

It is a natural phenomenon that when anisotropic turbulence decays, it becomes less anisotropic, and hence "returns to isotropy."

The second component, $\Phi_{i j, 2}$, is called the "rapid" pressure-strain redistribution, and it is modeled as

$$
\begin{aligned}
\Phi_{i j, 2}= & C_{2} \wp \rho a_{i j}+\left(C_{3 a}+C_{3 b} A_{2}^{1 / 2}\right) \rho k S_{i j} \\
& +C_{4} \rho k\left(a_{i k} S_{j k}+a_{j k} S_{i k}-\frac{2}{3} a_{k l} S_{k l} \delta_{i j}\right) \\
& +C_{5} \rho k\left(a_{i k} \Omega_{j k}+a_{j k} \Omega_{i k}\right)
\end{aligned}
$$

where

$$
\begin{aligned}
& S_{i j}=\frac{1}{2}\left(\frac{\partial U_{i}}{\partial x_{j}}+\frac{\partial U_{j}}{\partial x_{i}}\right) \equiv \text { mean rate-of-strain tensor } \\
& \Omega_{j k}=\frac{1}{2}\left(\frac{\partial U_{i}}{\partial x_{j}}-\frac{\partial U_{j}}{\partial x_{i}}\right) \equiv \text { mean rate-of-rotation tensor }
\end{aligned}
$$

and $\wp=u_{i} u_{k} \frac{\partial U_{i}}{\partial x_{k}}=\left(-\frac{1}{2 \rho}\right) P_{i i} \equiv$ half the trace of the production tensor

This term, Eq. (3.18), stems from rapid-distortion theory (RDT), which describes the limiting case where the turbulence-fluctuation-to-mean-strain-rate interaction is large, thus the name "rapid."

The last term in Eq.(3.14), $\Phi_{i j, w}$, is the wall-reflection term to account for the effect of solid walls. It is sometimes referred to as the pressure-echo term. Its purpose is to compensate for the large variation in the stress anisotropy when solids walls are present. This term, however, requires explicit calculations of the normal distance to the wall, and is not invariant to the coordinate system. The lack of invariance property is not suitable for 
applications involving moving walls, such as piston engines, for the flow domain and boundaries can change in time.

To eliminate the need for wall-reflection correction, Speziale, Sarkar and Gatski [1991] developed a quasi-linear model for $\Phi_{i j, 2}$ based on invariance considerations and dynamical systems approach. The model was successfully applied to homogeneous and wall-equilibrium flows, and is adopted for all research work in this dissertation.

\subsection{Dissipation-Rate Transport Equation}

Values of $\varepsilon$ are required to solve for the dissipation tensor, $\varepsilon_{i j}$, in Eq. (3.11). The exact partial-differential equation for the evolution of $\varepsilon$ is [Daly and Harlow, 1970]

$$
\begin{aligned}
\frac{D \varepsilon}{D t}= & -2 v \frac{\partial u_{i} \partial u_{i} \partial u_{k}}{\partial x_{k} \partial x_{l} \partial x_{l}}-2\left(v \frac{\partial^{2} u_{i}}{\partial x_{k} \partial x_{l}}\right)^{2}-\frac{\partial}{\partial x_{k}}\left[u_{k} \varepsilon^{\prime}+\frac{2 v}{\rho} \frac{\partial u_{k} \partial p}{\partial x_{l}} \frac{\partial}{\partial x}-v \frac{\partial \varepsilon}{\partial x_{k}}\right] \\
& -2 v\left[\frac{\partial u_{i} \partial u_{k}}{\partial x_{l} \partial x_{l}}+\frac{\partial u_{l} \partial u_{l}}{\partial x_{k} \partial x_{k}}\right] \frac{\partial U_{i}}{\partial x_{k}}-2 v u_{k} \frac{\partial u_{i} \partial^{2} U_{i}}{\partial x_{l} \partial x_{k} \partial x_{l}}
\end{aligned}
$$

Clearly, most terms in this equation need to be modeled. However, this exact expression for $\varepsilon$ does not serve as an appropriate basis for modeling. In the energy cascading process, at high Reynolds number, $\varepsilon$ is determined by large-scale eddy motions, unaffected by viscosity [Pope, 2000]. Therefore, Eq. (3.22) applies only to the low-Reynolds-number, or dissipative, region.

Conventionally, modeling is carried out via empirical and dimensional analyses.

The model equation for $\varepsilon$ proposed by workers in the 1970 s is often referred to as the “standard" model [Hanjalic and Launder, 1972; Launder and Sharma, 1974; Hanjalic and Launder, 1976]. Another approach to model the $\varepsilon$ equation is by employing the 
renormalization group (RNG) theory, in which the exact $k$ and $\varepsilon$ transport equations are renormalized systematically, resulting in the successive removal of high-wave-number bandwidths (i.e., small scales). This method was first developed by Wilson [1971] (a Nobel prize recipient in physics, 1982) to investigate critical phenomena in condensed matter physics. In applications to turbulence modeling, researchers who have utilized the RNG method include Yakhot and Orszag [1986], Smith and Reynolds [1992], and Han and Reitz [1995]. In this dissertation, however, the model equation for $\varepsilon$ is chosen to be the traditional, more-popular empirical form:

$$
\begin{aligned}
\frac{\partial}{\partial t} \rho \varepsilon+\frac{\partial}{\partial x_{k}}\left(\rho U_{k} \varepsilon\right)= & -\left(\frac{2}{3} C_{\varepsilon 1}-C_{\varepsilon 3}\right) \rho \varepsilon \frac{\partial U_{i}}{\partial x_{i}} \\
& +\frac{\partial}{\partial x_{k}}\left(\frac{\mu_{t}}{\sigma_{\varepsilon}} \frac{\partial \varepsilon}{\partial x_{k}}\right)-\rho \frac{\varepsilon}{k}\left(C_{\varepsilon 1} P+C_{\varepsilon 2} \varepsilon\right)
\end{aligned}
$$

Eq. (3.23) is based on the standard $\varepsilon$ model equation, with the first term on the right being the added source term. This extra contribution accounts for length scale changes due to velocity dilatation [Amsden et al., 1989].

\subsection{Modeling Constants}

The constants in all the model equations above are listed below:

Table 3.1. RSTM Model Constants

\begin{tabular}{|c|c|c|c|c|c|c|c|c|c|c|c|c|}
\hline$C_{1}$ & $C_{1 s}$ & $C_{2}$ & $C_{3 a}$ & $C_{3 b}$ & $C_{4}$ & $C_{5}$ & $C_{\varepsilon 1}$ & $C_{\varepsilon 2}$ & $C_{\varepsilon 3}$ & $C_{\mu}$ & $\sigma_{\kappa}$ & $\sigma_{\varepsilon}$ \\
\hline 1.7 & 1.05 & 0.9 & 0.8 & -.65 & .625 & 0.2 & 1.44 & 1.83 & -1.0 & 0.09 & 1.0 & 1.3 \\
\hline
\end{tabular}




\subsection{Wall Effects}

Unlike free-shear flows, in most turbulent flows encountered by engineers, the flow domain is usually partially or completely bounded, i.e., one or more solid surfaces are present. Both internal and external flows are examples of wall-flows: pipe and channel flow, and flow over an aircraft, race car, or ship. The walls can be of any shape - curvature, sudden expansion/contraction, etc. - and can therefore complicate the flow field. For fluid flow close to the wall, the effect of viscosity is pronounced. In addition, shear stress, which is related to the mean rate of strain, is significantly higher than in the region away from the wall.

The effects of solid boundaries in wall-bounded flows can be taken into account by two methods: (1). Direct integration of the transport equations (including the equations of motion as well as the model equations for turbulence) all the way to the wall, or (2). Application of wall functions. In the first method, model/transport equations are integrated throughout the entire flow domain including the solid wall boundaries. Exact boundary conditions are then imposed. Conventionally, turbulence models devised for such direct integration are referred to as "low-Reynolds-number models." This method, while being able to achieve higher accuracy and appearing to be fundamentally easy to implement, brings about difficulties in solving the equations. First, due to higher damping of fluctuations in the normal-to-wall than in the tangential directions, much denser grid is required (in the normal direction) close to the wall. This not only raises the computational overhead by a possibly large amount, the grid near the wall is consequently stretched, i.e., having very high aspect ratio. Second, in combination with any strong non-orthogonality, overly-stretched grid can cause serious numerical instability, and it can be detrimental to 
the solution convergence. Nevertheless, in flows where complex fluid structures, such as separation, non-equilibrium and strong inhomogeneity, are present, low-Reynolds-number modeling may be required to fully describe the flow details, e.g., wall shear stress and heat transfer, near the wall.

The second method to account for wall effects is by imposing the so-called wallfunctions. In contrast to the first method of direct integration of transport/model equations, the wall-function method bypasses the need for detailed computations of flow variables near the wall - particularly in the viscous sublayer where the viscous damping is large. Instead, functions utilizing empirical laws are used to bridge the flow between the wall and the outer boundary layer, where Reynolds number is higher than near the wall. Essentially, in this method, the need to resolve the steep flow variables, e.g., velocity gradients, has been replaced with the use of wall functions. By doing so, relatively coarser grid can be used in the near-wall region. Typically, the first grid point lies in the logarithmic-law-layer where the normal wall unit, $n^{+}$, is greater than 30 .

$$
\begin{aligned}
& n^{+} \text {is defined as } \\
& n^{+}=\frac{n}{\delta_{v}}
\end{aligned}
$$

where $n$ is the normal distance away from wall, and

$$
\delta_{v} \equiv \frac{v}{u_{\tau}} \equiv \text { viscous length scale }
$$

which is the ratio of molecular viscosity to the "friction velocity":

$$
u_{\tau} \equiv \sqrt{\frac{\tau_{w}}{\rho}}
$$

$\tau_{w}$ is the wall shear stress, given by 


$$
\tau_{w}=\left.\rho v \frac{d U}{d y}\right|_{y=0}
$$

which is contributed solely by the viscous effect.

A turbulence model that uses wall functions is typically referred to as a "highReynolds-number model." All formulations in this thesis work employ this modeling technique. The use of wall functions is justified here, since in the validation cases to be presented in Chapter 4 the predicted flow variables, e.g., Reynolds shear stress, match well with the DNS or experimental data in both turbulent and laminar (near-wall) regions. In addition, the low-Reynolds-number modeling method can result in higher computational cost (time and memory) as well as additional complexity in the mathematical modeling and solution procedure. However, it should be cautioned that the standard wall functions are most suitable for flows in energy equilibrium [Hadzic, 1999], and when flow details (e.g., pressure and velocity fluctuations) are not of primary interest. Early work in the development of wall functions did not yield satisfactory results [e.g., Launder and Spalding, 1973], but subsequent studies have been made, leading to a certain improvement in near-wall predictions. 


\section{Chapter 4}

\section{Implementation of RSTM in the KIVA code}

In this chapter, the numerical implementation of the Reynolds-stress turbulence model in the KIVA code will be discussed. A brief introduction of KIVA will first be given, followed by the differencing schemes used in the code. Then, the discretization of the Reynolds-stress transport equations will be presented, as well as the solution procedure, boundary conditions, handling of source terms, numerical issues, and code validation using experimental and DNS data for flows in a channel and a pipe. A description of the coding changes, e.g., additions and modifications to the KIVA code, will be included in Appendix A. 


\subsection{The KIVA Code}

The KIVA-3V (Release 2) computer code [Amsden et al., 1989; Amsden, 1993; Amsden, 1997; Amsden, 1999] is the latest released version of the KIVA-family CFD open-source codes developed by the Los Alamos National Laboratory (LANL) and is in use world-wide among the engine research communities. It solves transient, two- and three-dimensional, chemically reactive fluid flow with fuel sprays. Transport equations are written in Cartesian coordinates, and are solved in a multi-block computational domain subdivided into individual computational cells that are hexahedrons. Finite-volume approach is used to discretize the gas-phase equations. KIVA also allows cylindrical geometry, in which case a wrapped-around grid is used, in conjunction with periodic boundary condition in the azimuthal (tangential) direction. An arbitrary number of species can be included in the code, and the number of chemical reactions, both kinetic and equilibrium, is unlimited as well. It can be applied to either subsonic or supersonic flows, as well as laminar or turbulent flows. The turbulence models in the original code include both the standard [Launder and Spalding, 1974] and RNG-variant [Han and Reitz, 1995] $k-\varepsilon$ models. KIVA was originally written for internal-combustion engines, but it is also well-suited for through-flow-type combustors without any moving surfaces.

\subsubsection{The Governing Equations}

KIVA can handle flow fields with both gas and discrete liquid phases. The NavierStokes equation, in combination with the continuity, energy, and turbulence equations, are used to solve for the gas phase. The liquid phase refers to liquid sprays, typically fuel, described by a separate set of modeled partial-differential transport equations stochastic in 
nature. In addition, chemical reactions are solved by rate equations, which include both kinetic (of Arrhenius form) and equilibrium reactions. For velocity on the mesh boundaries, either no-slip, free-slip, or law-of-wall boundary conditions can be applied. For temperature, choices include fixed-temperature or adiabatic walls. Periodic boundary condition is applied for cylindrical grids. Additionally, to account for liquid droplet sprays, a spray-injector boundary condition is used. For inflow and outflow boundaries, either velocity or pressure can be specified.

With Reynolds-stress turbulence model, the six components of Reynolds-stresses can now directly be used in the RANS equation, Eq. (2.7), repeated here:

$$
\frac{\partial}{\partial t}\left(\rho U_{i}\right)+\frac{\partial}{\partial x_{j}}\left(\rho U_{i} U_{j}\right)=-\frac{\partial p}{\partial x_{i}}-\frac{\partial}{\partial x_{j}}\left(\rho u_{i} u_{j}\right)+\frac{\partial \tau_{i j}}{\partial x_{j}}
$$

where

$$
\tau_{i j}=\mu\left(\frac{\partial U_{i}}{\partial x_{j}}+\frac{\partial U_{j}}{\partial x_{i}}-\frac{2}{3} \frac{\partial U_{k}}{\partial x_{k}} \delta_{i j}\right)
$$

$\mu$ being the molecular viscosity.

All remaining equations in KIVA are unaffected and can be found in the user's manual of KIVA-II [Amsden et al., 1989].

\subsubsection{The Numerical Scheme}

KIVA uses the Arbitrary Lagrangian-Eulerian (ALE) method [Hirt et al., 1974; Pracht, 1975]. The solution procedure is divided into the Lagrangian ("Phases A \& B") and rezone ("Phase C") stages within each computational cycle. In the Lagrangian phases, the computational cells move with the fluid, and essentially all but the convection terms in all the transport equations are solved here. 
Phase A calculates the spray dynamics, including collision, oscillation, breakup, and evaporation. In addition, all source terms due to spray and chemistry in the mass, energy and turbulence equations are solved in this phase. At the end of Phase A, all computed quantities are updated with the intermediate values, to be accompanied by the subscript "A."

In Phase B, the acoustic mode terms are solved. These terms include the pressure gradient in the momentum equation and velocity dilatation terms in the mass and energy equations. In addition, the diffusion terms in the momentum and energy equations are also calculated in this phase. These terms are discretized in a semi-implicit, coupled fashion. The remaining source terms in the turbulence equations are also calculated in this phase. These terms are solved using the conjugate residual iterative method [O'Rourke and Amsden, 1986]. Upon completion of Phase B, all pertinent quantities are updated and represented by the subscript "B."

In Phase C, the entire flow field is "frozen" while the computational domain is remapped onto a new mesh, be it a different location from the previous time step as specified by the user, or the original position (e.g., stationary grids such as that of gas turbine combustors). The convective transport due to this relative movement between the fluid and cells is calculated in this phase. The numerical methods available include partial donor cell and quasi-second-order upwind schemes. A total-variation-diminishing (TVD) scheme has also been incorporated as an option.

Completion of Phase $\mathrm{C}$ also signifies completion of the cycle. All quantities are updated with the final values, and carry the subscript " $n+1$," since it is equivalent to the subscript "C." 
The flow domain of interest is divided into discrete subregions, called "cells," that are hexahedron in shape. These cells can deform arbitrarily while maintaining their logical hexahedron structure and being subject to numerical stability and accuracy, depending on the current solution phase (i.e., A, B, or C). All thermodynamic quantities, such as pressure, density, temperature and Reynolds-stresses, are stored at the cell centers. Vector variables, namely the three components of velocity, are defined at cell corners, called "vertices." This creates a grid system that is staggered, eliminating the need to interpolate vertex velocity. More detail on the advantages and disadvantages of staggered grid can be found in the references herein.

\section{Temporal Differencing}

Any quantities $(Q)$ partial differential with respect to time is approximated by the first-order Euler approximation:

$$
\frac{\partial Q}{\partial t}=\frac{Q^{n+1}-Q^{n}}{\Delta t}
$$

where $\Delta t$ is the time step-size and the integer $n$ is the cycle number. The time step-size needs not be constant from one cycle to the next. Instead, it is determined based on certain accuracy (Phase B) and stability (Phase C) criteria. In Phase B, since the solution procedure is largely implicit, the time step-size for each cycle is restricted by accuracy in the calculations of fluid acceleration $\left(\Delta t_{a c c}\right)$, rate of strain $\left(\Delta t_{r s t}\right)$, chemical kinetic reactions $\left(\Delta t_{c h}\right)$, spray momentum $\left(\Delta t_{s p}\right)$, spark ignition interval $\left(\Delta t_{i g n}\right)$ and fuel injection interval $\left(\Delta t_{i n j}\right)$. That is,

$$
\Delta t_{\text {Phase B }}=\min \left(\Delta t_{a c c}, \Delta t_{r s t}, \Delta t_{c h}, \Delta t_{s p}, \Delta t_{i g n}, \Delta t_{i n j}\right)
$$


Phase $\mathrm{C}$, on the other hand, is performed in a subcycled and fully-explicit fashion. It is, therefore, subject to the Courant stability condition, which in general limits the flux volume to be less than the cell volume. This convective time-step constraint subsequently determines the number of convective subcycles, $N S$, typically equal to one or two. The time-step used in Phase $\mathrm{C}$ is therefore

$$
\Delta t_{\text {Phase C }}=\frac{\Delta t}{N S}
$$

Each cycle is performed in three stages (phases) as illustrated in Figure 4.1.

\section{Spatial Differencing}

KIVA employs the finite-volume, Arbitrary Lagrangian-Eulerian (ALE) approach to preserve the local conservative properties. Each computational cell contains eight nodes (or "vertices"), six faces, and twelve edges, all of which are shared with neighboring cells (except for boundary cells). The cells may move arbitrarily and, therefore, may experience distortion during the Lagrangian phase.

Spatial differencing is accomplished by using the finite-volume method. A differential equation for any transported quantity $Q$, which can be either a scalar, vector or tensor, with flux and source terms, can be written as

$$
\frac{\partial Q}{\partial t}+\nabla \cdot \vec{F}=S
$$

Integrating over each of the control volume within the entire three-dimensional flow domain,

$$
\int_{V}\left[\frac{\partial Q}{\partial t}+\nabla \bullet \vec{F}=S\right] d V
$$


$n^{\text {th }}$ time step: $Q^{n}, Q=(\rho, u, v, w, T, \ldots)$

Phase A (Lagrangian):

- Calculates spray droplet collision and oscillation/ breakup terms

- Mass and energy source terms due to the chemistry and spray

- Auxiliary quantities at the end of Phase A: $Q^{A}$

Phase B (Lagrangian, control volume moves with fluid $\vec{u}$ ):

- Solves the governing equations in a coupled, implicit fashion with individual eqs solved by the conjugate residual method [O'Rourke and Amsden, 1986]

- The procedure is very similar to the SIMPLE scheme [Patankar, 1980]

- $\Delta t$ is determined based on accuracy, not stability

\section{CYCLE}

- Calculates:

(a) acoustic mode terms, namely the pressure gradient in the momentum equation and velocity dilatation terms in mass and energy equations

(b) the spray momentum source term

(c) the diffusion terms (mass, momentum, energy)

(d) the remaining source terms in $k$ and $\varepsilon$ equations

- Auxiliary quantities at the end of Phase B: $Q^{B}$

Phase C (Rezone/Remap):

- The flow field is frozen and rezoned or remapped onto a new computational mesh

- Calculates the convective transport associated with moving the mesh relative to the fluid

- This is accomplished in a subcycled, fully explicit fashion

- $\Delta t_{c}$ is determined based on stability

- Quantities at the end of Phase C: $Q^{C}=Q^{n+1}$

Figure 4.1. The Three Phases in KIVA 
Applying divergence theorem, the volume integral of the divergence of flux term can be converted into surface integral, where the integration is carried out over the six faces (or control surface) of each control volume. Thus, the integral form of Eq. (4.6) is

$$
\frac{\partial}{\partial t} \int_{V} Q d V+\int_{A} \vec{F} \bullet d \vec{A}=\int_{V} S d V
$$

There are three types of control volume (or cell) in KIVA, each of which serves a different purpose. They are regular cell, momentum cell, and normal-velocity-centeredon-cell-face cell.

\section{(1) Regular Cell}

In the layout of the computational mesh, the actual nodes, or vertices, constitute regular cells. Each regular cell, therefore, consists of eight vertex points and six faces, as illustrated in the schematic drawing below, Figure 4.2. This figure also shows the orientation of the coordinate system. A regular cell is designated "cell ijk," and the vertex having the smallest $(x, y, z)$ values, in the left-front-bottom corner, is called 'vertex ijk.' This arbitrarily shaped hexahedron may be moved in an arbitrarily prescribed manner, a feature of the ALE method.

Regular cells are used to store thermodynamic and turbulence properties, including pressure $p$, species density $\rho_{m}$, total fluid density $\rho$, temperature $T$, internal energy $I$, turbulent kinetic energy $k$, and its dissipation rate $\varepsilon$. Reynolds-stresses, $u_{i} u_{j}$, are also stored in regular cells. These quantities are located at the cell centers, and are constant within the cell. In addition, vector quantities, i.e., the three components of velocity, $u, v$, and $w$, are stored at the cell vertices. 


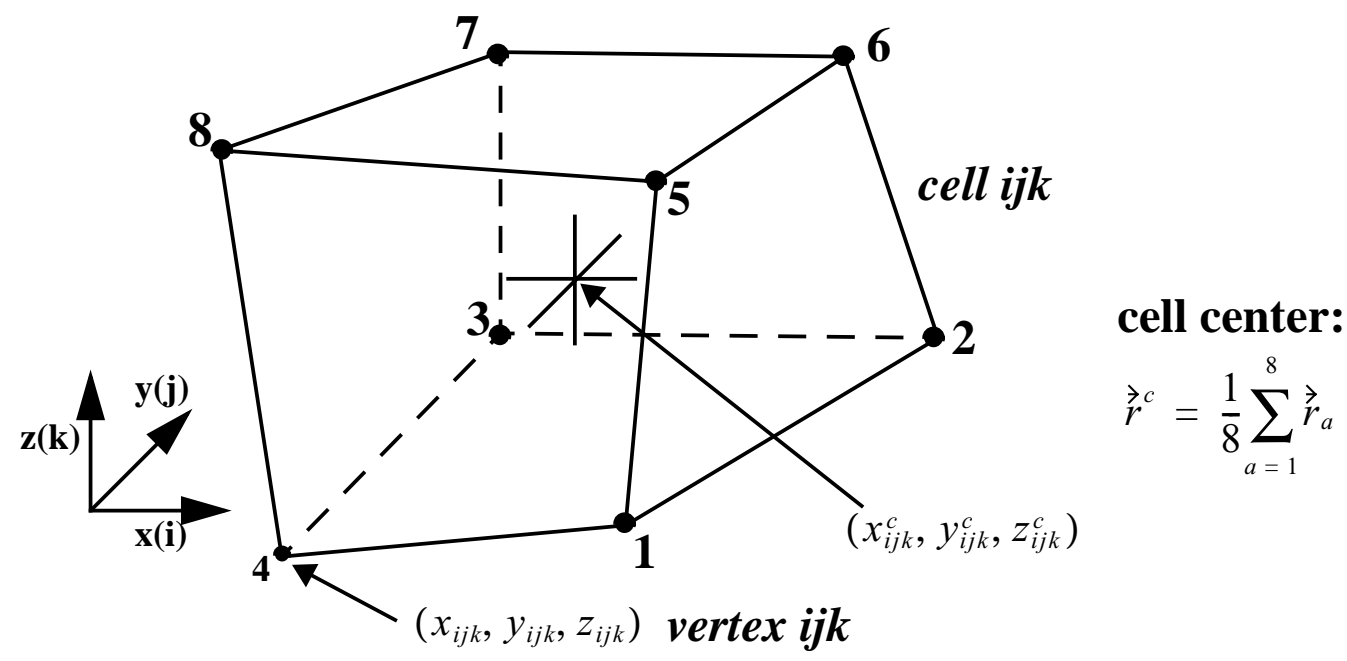

Figure 4.2. A Regular Cell Layout

The position vector to vertex $(i, j, k)$ is

$$
\vec{x}_{i j k}=x_{i j k} \vec{i}+y_{i j k} \vec{j}+z_{i j k} \vec{k}
$$

and the center of cell ijk is defined as the point with coordinates

$$
x_{i j k}^{c}=\frac{1}{8} \sum_{\alpha=1}^{8} x_{\alpha} \quad y_{i j k}^{c}=\frac{1}{8} \sum_{\alpha=1}^{8} y_{\alpha} \quad z_{i j k}^{c}=\frac{1}{8} \sum_{\alpha=1}^{8} z_{\alpha}
$$

which is the geometric average of the eight vertices of a regular cell. This cell center is exactly where the thermodynamic and turbulence quantities are located, i.e.,

$$
Q_{i j k}=Q\left(x_{i j k}^{c}, y_{i j k}^{c}, z_{i j k}^{c}\right)
$$

Each regular cell has three associated characteristic cell faces, namely left, front, and bottom, as shown in Figure 4.3. The remaining three faces are, in turn, the characteristic cell faces associated with the neighboring cells. 


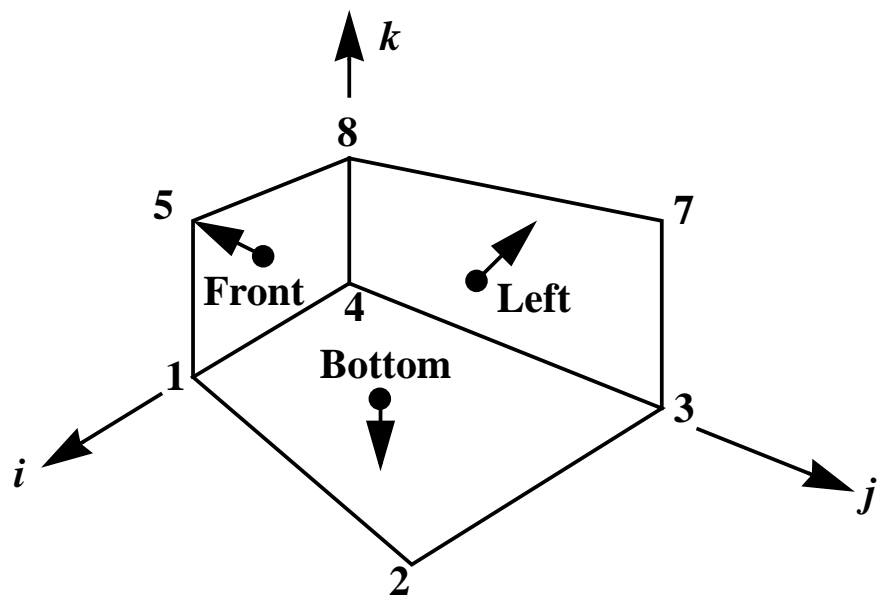

Figure 4.3. Three Cell Faces Associated with Cell ijk

\section{(2) Momentum Cell}

The second type of control volume is the momentum cell. It is used, as its name suggests, for the discretization of momentum equation. The $(i j k)^{t h}$ momentum cell is centered about the $(i j k)^{t h}$ vertex of the $(i j k)^{t h}$ regular cell, as shown in Figure 4.4 where the location of a momentum cell is illustrated by using a two-dimensional grid for ease of visualization.

2-D:

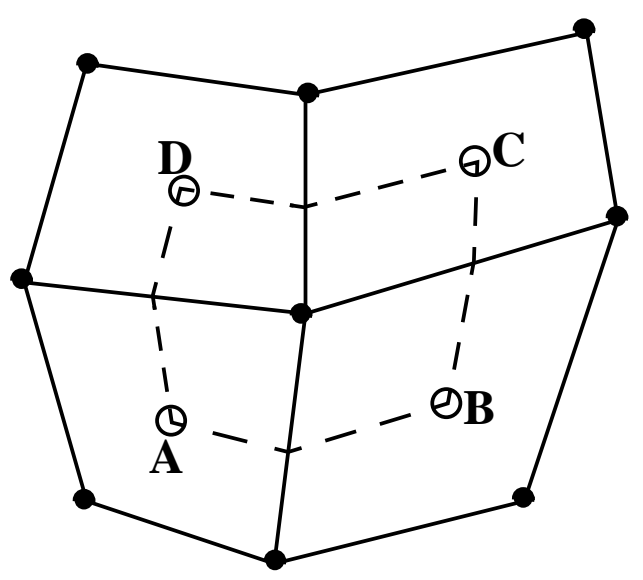

Figure 4.4. Momentum Cell $i j$, in Dashed Lines, and Its Associated Regular Cells 
Unlike regular cell, a momentum cell has twenty four cell faces, three of which are embedded within a regular cell. The shaded areas in Figure 4.4 represent such three faces, which consist of one-eighth of a full momentum cell.

Since the velocity vector, $\vec{u}$, is located at the regular cell vertex, i.e.,

$$
\vec{u}_{i j k}=\vec{u}\left(x_{i j k}, y_{i j k}, z_{i j k}\right)
$$

represented by filled circles in Figures 4.4 and 4.5, no interpolation is necessary for the calculation of vertex movement.

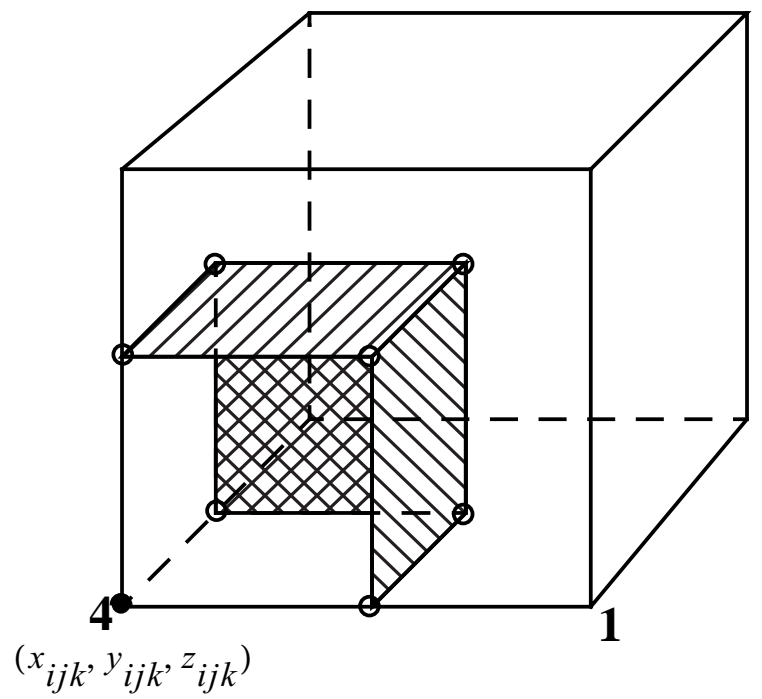

Figure 4.5. The Portion of Momentum Cell ijk Lying Within Regular Cell ijk

\section{(3) Normal-Velocity-Centered-on-Cell-Face (NVCOCF) Cell}

The last type of control volume is used to calculate the cell volume changes in Phase B for the acoustic subcycling and in Phase $\mathrm{C}$ for the convective fluxing. Similar to the staggered grid used in the SIMPLE-type [Patankar, 1980] schemes, these NVCOCF cells are offset in each of the logical directions by half regular cell-width, as shown in 
Figure 4.6, where thin lines represent regular cell boundaries, while heavy lines delineate the NVCOCF cell. The arrow shows the location and direction of the newly computed normal velocity.

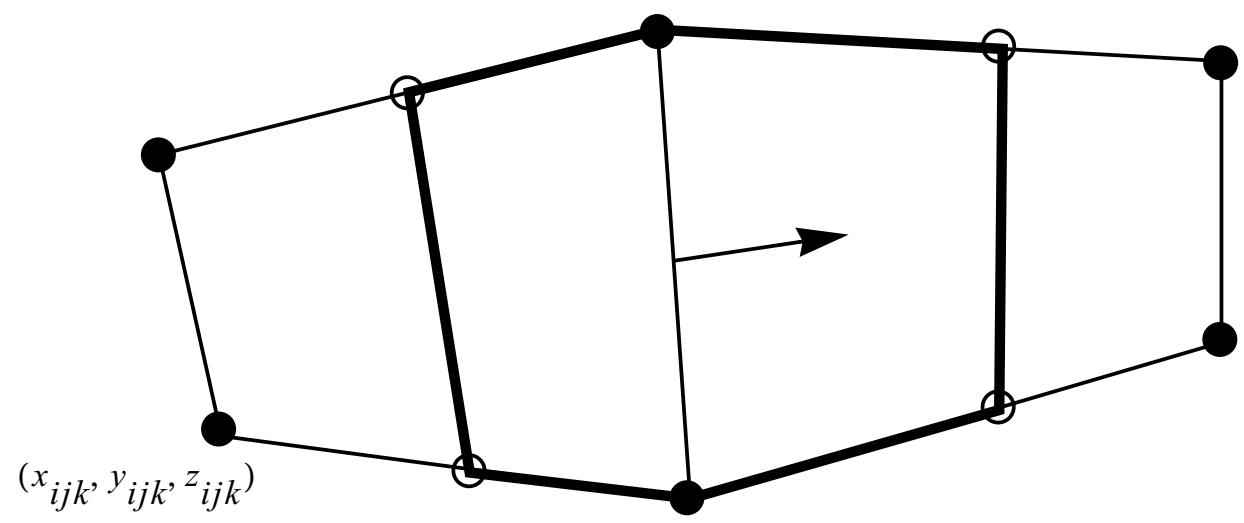

Figure 4.6. 2-D Schematic of a NVCOCF Cell

The NVCOCF cell is used to calculate the accelerations of the face-centered normal velocity, $\vec{u}_{a}$ due to the thermodynamic pressure $p$ and turbulence pressure $\frac{2}{3} \rho k$ in Phase B. It is also used for the calculations of cell volume changes in Phase B as well as fluxing volume in Phase C.

\subsubsection{Solution Procedure in Phase B}

The Phase B calculations of pressure field and diffusion are based on the SIMPLE scheme [Patankar, 1980]. It is essentially a two-step procedure in which a "predicted" pressure field is assumed and used to compute the diffusion terms in the momentum and energy equations, the volume change equation as well as the equation of state. These quantities are in turn used to solve for the "corrected" pressure. Convergence is reached 
when the convergence criteria is satisfied. Otherwise, the two-step procedure, or "outer iteration," is repeated setting the newly computed (corrected) pressure as the predicted pressure. The individual equation in this outer iteration is solved by the use of conjugate residual iterative method [O'Rourke and Amsden, 1986].

\subsection{Discretization of Reynolds-Stress Transport Equations}

Reynolds-stresses, like all other thermodynamic quantities in KIVA, are located at cell-centers. The governing equations are discretized using the finite-volume method (FVM). Accordingly, the transport equations for the Reynolds stresses are discretized as described below.

For each of the computational cell, the Reynolds-stress transport equation is first integrated over the cell volume $d V$. For the Lagrangian Phase B solution, all but the convection terms are calculated. The integration is therefore

$$
\int_{V}\left[\frac{\partial}{\partial t}\left(\rho u_{i} u_{j}\right)=P_{i j}+\varepsilon_{i j}+\Phi_{i j}+D_{i j}\right] d V
$$

Each term is treated as follows:

\section{Unsteady Term}

As mentioned in a previous section, the temporal differencing is performed using the first-order Euler approximation, i.e.,

$$
\int_{V}\left[\frac{\partial}{\partial t}\left(\rho u_{i} u_{j}\right)\right] d V=\frac{M^{B}\left(u_{i} u_{j}\right)^{B}-M^{n}\left(u_{i} u_{j}\right)^{n}}{\Delta t}
$$

where $M$ is the mass of the fluid mixture in a cell (taken to be uniform within the cell), and $\Delta t$ is the discrete time step-size. The superscript $B$ denotes the quantities at the end of 
Phase B, i.e., the unknown, whereas superscript $n$ represents the known quantities at the current time.

\section{Production}

Since the production of turbulent kinetic energy, $P_{i j}$, is generally positive, it is evaluated in an explicit manner, i.e.,

$$
\begin{aligned}
\int_{V}\left[P_{i j}\right] d V & =\int_{V}\left[-\left(\rho u_{i} u_{k} \frac{\partial U_{j}}{\partial x_{k}}+\rho u_{j} u_{k} \frac{\partial U_{i}}{\partial x_{k}}\right)\right] d V \\
& =-M^{B}\left[\left(u_{i} u_{k}\right)^{n}\left(\frac{\partial U_{j}}{\partial x_{k}}\right)^{n}+\left(u_{j} u_{k}\right)^{n}\left(\frac{\partial U_{i}}{\partial x_{k}}\right)^{n}\right]
\end{aligned}
$$

\section{Dissipation}

Since the dissipation of turbulent kinetic energy, $\varepsilon_{i j}$, is always negative, it is treated explicitly:

$$
\begin{aligned}
\int_{V}\left[\varepsilon_{i j}\right] d V & =\int_{V}\left[-\frac{2}{3} \rho \varepsilon \delta_{i j}\right] d V \\
& =M^{B}\left(-\frac{2}{3} \varepsilon^{n}\right) \delta_{i j}
\end{aligned}
$$

\section{Pressure-Strain Redistribution}

The treatment of the pressure-rate-of-strain tensor, $\Phi_{i j}$, is also similar to production and dissipation:

$$
\begin{aligned}
\int_{V}\left[\Phi_{i j}\right] d V & =\int_{V}\left[\Phi_{i j, 1}+\Phi_{i j, 2}\right] d V \\
& =M^{B}\left[\frac{1}{\rho} \Phi_{i j, 1}+\frac{1}{\rho} \Phi_{i j, 2}\right]^{n}
\end{aligned}
$$

where $\Phi_{i j, 1}$ and $\Phi_{i j, 2}$ were defined in Chapter 3, Eq. (3.15) and Eq. (3.18). 


\section{Diffusion}

The diffusion term, $D_{i j}$, is treated in a way similar to how $k$, the turbulent kinetic energy, is handled in KIVA's two-equation models:

$$
\begin{aligned}
\int_{V}\left[D_{i j}\right] d V & =\int_{V}\left[\frac{\partial}{\partial x_{k}}\left(\frac{\mu_{t}}{\sigma_{\kappa}} \frac{\partial}{\partial x_{k}}\left(u_{i} u_{j}\right)\right)\right] d V \\
& =\sum_{a} \frac{\mu_{t}}{\sigma_{\kappa}} \nabla[\underbrace{}_{\text {Implicit }} \underbrace{\phi^{D}\left(u_{i} u_{j}\right)^{B}}_{\text {Explicit }}+\left(1-\phi^{D}\right)\left(u_{i} u_{j}\right)^{n}]_{a} \bullet A_{a}
\end{aligned}
$$

where $\phi^{D}$ is the variable implicitness parameter for diffusion, ranging from 0.0 (fullyexplicit) to 1.0 (fully implicit). This parameter is calculated internally in KIVA, and varies in space and time. Its value depends on the local diffusion number:

$$
C_{d}=\frac{\mu}{\rho} \frac{\Delta t}{(\Delta x)^{2}}
$$

The inclusion of $\phi^{D}$ in the differencing formulation is to enhance numerical stability [Amsden, 1989].

In Eq. (4.18), divergence theorem has been employed to convert the volume integral to area integral. The subscript $a$ represents each of the six faces of a regular cell, and $\boldsymbol{A}_{a}$ is the area vector (outward pointing) of such faces.

Grouping Eqs. (4.14-4.18), the discretized Reynolds-stress turbulence equation can be written: 


$$
\begin{aligned}
\frac{M^{B}\left(u_{i} u_{j}\right)^{B}-M^{n}\left(u_{i} u_{j}\right)^{n}}{\Delta t}= & -M^{B}\left[\left(u_{i} u_{k}\right)^{n}\left(\frac{\partial U_{j}}{\partial x_{k}}\right)^{n}+\left(u_{j} u_{k}\right)^{n}\left(\frac{\partial U_{i}}{\partial x_{k}}\right)^{n}\right] \\
& +M^{B}\left(-\frac{2}{3} \varepsilon^{n}\right) \delta_{i j} \\
& +M^{B}\left[\frac{1}{\rho} \Phi_{i j, 1}+\frac{1}{\rho} \Phi_{i j, 2}\right]^{n} \\
& +\sum_{a} \frac{\mu_{t}}{\sigma_{\kappa}} \nabla\left[\phi^{D}\left(u_{i} u_{j}\right)^{B}+\left(1-\phi^{D}\right)\left(u_{i} u_{j}\right)^{n}\right]_{a} \bullet A_{a}
\end{aligned}
$$

Rearranging,

$$
\begin{aligned}
\left(u_{i} u_{j}\right)^{B}= & -(\Delta t)\left[\left(u_{i} u_{k}\right)^{n}\left(\frac{\partial U_{j}}{\partial x_{k}}\right)^{n}+\left(u_{j} u_{k}\right)^{n}\left(\frac{\partial U_{i}}{\partial x_{k}}\right)^{n}\right] \\
& +(\Delta t)\left(-\frac{2}{3} \varepsilon^{n}\right) \delta_{i j} \\
& +(\Delta t)\left[\frac{1}{\rho} \Phi_{i j, 1}+\frac{1}{\rho} \Phi_{i j, 2}\right]^{n} \\
& +\left(\frac{\Delta t}{M^{B}}\right) \sum_{a} \frac{\mu_{t}}{\sigma_{\mathrm{\kappa}}} \nabla\left[\phi^{D}\left(u_{i} u_{j}\right)^{B}+\left(1-\phi^{D}\right)\left(u_{i} u_{j}\right)^{n}\right]_{a} \bullet A_{a} \\
& +\left(u_{i} u_{j}\right)^{n}
\end{aligned}
$$

\subsection{Discretization of Dissipation-Rate Equation}

Similar to the Reynolds-stress equation, the transport equation for the dissipation rate of turbulent kinetic energy, Eq. (3.23), is discretized using the finite-volume method. This equation is integrated over the cell control volume $V$, i.e.,

$$
\int_{V}\left[\frac{\partial}{\partial t} \rho \varepsilon=\vartheta_{\varepsilon}+P_{\varepsilon}+\varepsilon_{\varepsilon}+D_{\varepsilon}\right] d V
$$

where 


$$
\begin{aligned}
\vartheta_{\varepsilon} & =-\left(\frac{2}{3} C_{\varepsilon 1}-C_{\varepsilon 3}\right) \rho \varepsilon \frac{\partial U_{i}}{\partial x_{i}} \\
& =-\left(\frac{2}{3} C_{\varepsilon 1}-C_{\varepsilon 3}\right) \rho \varepsilon\left(\frac{\partial U}{\partial x}+\frac{\partial V}{\partial y}+\frac{\partial W}{\partial z}\right)
\end{aligned}
$$

is the dilatation, and

$$
P_{\varepsilon}=-\rho \frac{\varepsilon}{k}\left(C_{\varepsilon 1} \wp\right)
$$

is the production, where

$$
\begin{aligned}
\wp & =u_{i} u_{k} \frac{\partial U_{i}}{\partial x_{k}}=\left(-\frac{1}{2 \rho}\right) P_{i i} \\
\varepsilon_{\varepsilon} & =-\rho \frac{\varepsilon}{k}\left(C_{\varepsilon 2} \varepsilon\right)
\end{aligned}
$$

represents the dissipation, and

$$
D_{\varepsilon}=\frac{\partial}{\partial x_{k}}\left(\frac{\mu_{t}}{\sigma_{\varepsilon}} \frac{\partial \varepsilon}{\partial x_{k}}\right)=\frac{\partial}{\partial x}\left(\frac{\mu_{t}}{\sigma_{\varepsilon}} \frac{\partial \varepsilon}{\partial x}\right)+\frac{\partial}{\partial y}\left(\frac{\mu_{t}}{\sigma_{\varepsilon}} \frac{\partial \varepsilon}{\partial y}\right)+\frac{\partial}{\partial z}\left(\frac{\mu_{t}}{\sigma_{\varepsilon}} \frac{\partial \varepsilon}{\partial z}\right)
$$

is the diffusion. Each source term is treated and linearized as follows:

1. Unsteady Term

Temporal differencing for dissipation, as in KIVA, uses Euler's first-order approximation:

$$
\int_{V}\left[\frac{\partial}{\partial t}(\rho \varepsilon)\right] d V=\frac{M^{B}(\varepsilon)^{B}-M^{n}(\varepsilon)^{n}}{\Delta t}
$$

\section{Velocity Dilatation}

The velocity dilatation term, an added source term in KIVA due to length scale changes in moving grids, is linearized as follows: 


$$
\int_{V}\left[\vartheta_{\varepsilon}\right] d V=\int_{V}\left[-\left(\frac{2}{3} C_{\varepsilon 1}-C_{\varepsilon 3}\right) \rho \varepsilon(\nabla \bullet \boldsymbol{U})\right] d V
$$

Applying divergence theorem, the volume integral can be converted into an area integral. Therefore, Eq. (4.29) can be re-written as

$$
\int_{V}\left[\vartheta_{\varepsilon}\right] d V=\int_{A}\left[-\left(\frac{2}{3} C_{\varepsilon 1}-C_{\varepsilon 3}\right) \rho \varepsilon\right](\boldsymbol{U} \bullet d \boldsymbol{A})
$$

Since

$$
\frac{D V}{D t}=\int_{A} \boldsymbol{U} \bullet d \boldsymbol{A}
$$

Eq. (4.30) can be expressed as

$$
\int_{V}\left[\vartheta_{\varepsilon}\right] d V=-\left(\frac{2}{3} C_{\varepsilon 1}-C_{\varepsilon 3}\right) \rho^{B}\left(\frac{V^{B}-V^{n}}{\Delta t}\right)\left[(1-f) \varepsilon^{n}+f \varepsilon^{B}\right]
$$

where

$$
f= \begin{cases}1 & \text { if } V^{B}-V^{n}>0 \\ 0 & \text { otherwise }\end{cases}
$$

The function $f$ is devised to circumvent any negative computed values of $\varepsilon$ when large changes of volume $V$ are present during the Lagrangian phase.

Eq. (4.32) can alternatively be expressed as

$$
\int_{V}\left[\vartheta_{\varepsilon}\right] d V=-\left(\frac{2}{3} C_{\varepsilon 1}-C_{\varepsilon 3}\right) \rho^{B}\left\{\left(\frac{V^{B}-V^{n}}{\Delta t}\right) \varepsilon^{n}+\max \left[\left(\frac{V^{B}-V^{n}}{\Delta t}\right), 0.0\right]\left(\varepsilon^{B}-\varepsilon^{n}\right)\right\}
$$

or 


$$
\begin{aligned}
\int_{V}\left[\vartheta_{\varepsilon}\right] d V= & -\left(\frac{2}{3} C_{\varepsilon 1}-C_{\varepsilon 3}\right) \rho^{B}\left\{\left(\frac{V^{B}-V^{n}}{\Delta t}\right)-\max \left[\left(\frac{V^{B}-V^{n}}{\Delta t}\right), 0.0\right]\right\} \varepsilon^{n} \\
& -\left(\frac{2}{3} C_{\varepsilon 1}-C_{\varepsilon 3}\right) \rho^{B}\left\{\max \left[\left(\frac{V^{B}-V^{n}}{\Delta t}\right), 0.0\right]\right\} \varepsilon^{B}
\end{aligned}
$$

\section{Production}

Since the production term is generally positive, explicit formulation is used:

$$
\begin{aligned}
\int_{V}\left[P_{\varepsilon}\right] d V & =\int_{V}\left[-\rho \frac{\varepsilon}{k}\left(C_{\varepsilon 1} \wp\right)\right] d V \\
& =M^{B}\left(\frac{\varepsilon}{k}\right)^{n}\left(-C_{\varepsilon 1} \wp^{n}\right)
\end{aligned}
$$

\section{Dissipation}

Using Taylor series expansion (first Taylor polynomial) for local linearization, the dissipation term can be expressed as

$$
\varepsilon_{\varepsilon}^{B}=\varepsilon_{\varepsilon}^{n}+\left(\frac{\partial \varepsilon_{\varepsilon}}{\partial \varepsilon}\right)^{n}\left(\varepsilon^{B}-\varepsilon^{n}\right)
$$

where

$$
\frac{\partial \varepsilon_{\varepsilon}}{\partial \varepsilon}=\frac{\partial}{\partial \varepsilon}\left[\rho \frac{\varepsilon}{k}\left(-C_{\varepsilon 2} \varepsilon\right)\right]=2\left[\rho \frac{\varepsilon}{k}\left(-C_{\varepsilon 2}\right)\right]
$$

This yields

$$
\begin{aligned}
\varepsilon_{\varepsilon}^{B} & =\left[\rho \frac{\varepsilon}{k}\left(-C_{\varepsilon 2} \varepsilon\right)\right]^{n}-2\left(\rho \frac{\varepsilon}{k}\left(-C_{\varepsilon 2}\right)\right)^{n} \varepsilon^{n}+2\left(\rho \frac{\varepsilon}{k}\left(-C_{\varepsilon 2}\right)\right)^{n} \varepsilon^{B} \\
& =\left[\rho \frac{\varepsilon}{k}\left(C_{\varepsilon 2} \varepsilon\right)\right]^{n}+2\left(\rho \frac{\varepsilon}{k}\left(-C_{\varepsilon 2}\right)\right)^{n} \varepsilon^{B}
\end{aligned}
$$

Alternatively, treating $\frac{\varepsilon}{k}$ as a constant locally [Cline and Yang, 1996] 


$$
\frac{\partial \varepsilon_{\varepsilon}}{\partial \varepsilon}=\left[\rho \frac{\varepsilon}{k}\left(-C_{\varepsilon 2}\right)\right] \frac{\partial \varepsilon}{\partial \varepsilon}=\rho \frac{\varepsilon}{k}\left(-C_{\varepsilon 2}\right)
$$

Consequently,

$$
\begin{aligned}
\varepsilon_{\varepsilon}^{B} & =\left[\rho \frac{\varepsilon}{k}\left(-C_{\varepsilon 2} \varepsilon\right)\right]^{n}-\left(\rho \frac{\varepsilon}{k}\left(-C_{\varepsilon 2}\right)\right)^{n} \varepsilon^{n}+\left(\rho \frac{\varepsilon}{k}\left(-C_{\varepsilon 2}\right)\right)^{n} \varepsilon^{B} \\
& =\left(\rho \frac{\varepsilon}{k}\left(-C_{\varepsilon 2}\right)\right)^{n} \varepsilon^{B}
\end{aligned}
$$

Integrating over the regular cell volume $d V$,

$$
\begin{aligned}
\int_{V}\left[\varepsilon_{\varepsilon}\right] d V & =\int_{V}\left[\left(\rho \frac{\varepsilon}{k}\left(-C_{\varepsilon 2}\right)\right)^{n} \varepsilon^{B}\right] d V \\
& =M^{B}\left(\frac{\varepsilon}{k}\left(-C_{\varepsilon 2}\right)\right)^{n} \varepsilon^{B}
\end{aligned}
$$

\section{Diffusion}

Using the implicitness parameter described in the previous section, the diffusion term in the $\varepsilon$ transport equation is discretized as

$$
\begin{aligned}
\int_{V}\left[D_{\varepsilon}\right] d V & =\int_{V}\left[\frac{\partial}{\partial x_{k}}\left(\frac{\mu_{t}}{\sigma_{\varepsilon}} \frac{\partial \varepsilon}{\partial x_{k}}\right)\right] d V \\
& =\sum_{a} \frac{\mu_{t}}{\sigma_{\varepsilon} \nabla\left[\phi^{D} \varepsilon^{B}+\right.}+\underbrace{\left.\left(1-\phi^{D}\right) \varepsilon^{n}\right]_{a} \bullet \boldsymbol{A}_{a}}_{\text {Implicit }}
\end{aligned}
$$

Summing Eqs. (4.28-4.42), the discretized transport equation for the dissipation of turbulent kinetic energy is 


$$
\begin{aligned}
\frac{M^{B}(\varepsilon)^{B}-M^{n}(\varepsilon)^{n}}{\Delta t}= & -\left(\frac{2}{3} C_{\varepsilon 1}-C_{\varepsilon 3}\right) \rho^{B}\left\{\left(\frac{V^{B}-V^{n}}{\Delta t}\right)-\max \left[\left(\frac{V^{B}-V^{n}}{\Delta t}\right), 0.0\right]\right\} \varepsilon^{n} \\
& +\left[-\left(\frac{2}{3} C_{\varepsilon 1}-C_{\varepsilon 3}\right) \rho^{B}\left\{\max \left[\left(\frac{V^{B}-V^{n}}{\Delta t}\right), 0.0\right]\right\} \varepsilon^{B}\right] \\
& +M^{B}\left(\frac{\varepsilon}{k}\right)^{n}\left(-C_{\varepsilon 1} \wp^{n}\right) \\
& +M^{B}\left(\frac{\varepsilon}{k}\left(-C_{\varepsilon 2}\right)\right)^{n} \varepsilon^{B} \\
& +\sum_{a} \frac{\mu_{t}}{\sigma_{\varepsilon}} \nabla\left[\phi^{D} \varepsilon^{B}+\left(1-\phi^{D}\right) \varepsilon^{n}\right]_{a} \bullet \boldsymbol{A}_{a}
\end{aligned}
$$

Rearranging yields

$$
\begin{aligned}
\varepsilon^{B}= & -\left(\frac{2}{3} C_{\varepsilon 1}-C_{\varepsilon 3}\right)\left(\frac{1}{V}\right)^{B}\left\{\left(V^{B}-V^{n}\right)-\max \left[\left(V^{B}-V^{n}\right), 0.0\right]\right\} \varepsilon^{n} \\
& +\left[-\left(\frac{2}{3} C_{\varepsilon 1}-C_{\varepsilon 3}\right)\left(\frac{1}{V}\right)^{B}\left\{\max \left[\left(V^{B}-V^{n}\right), 0.0\right]\right\} \varepsilon^{B}\right] \\
& +\Delta t\left(\frac{\varepsilon}{k}\right)^{n}\left(-C_{\varepsilon 1} \wp^{n}\right) \\
& +\Delta t\left(\frac{\varepsilon}{k}\left(-C_{\varepsilon 2}\right)\right)^{n} \varepsilon^{B} \\
& +\frac{\Delta t}{M^{B}} \sum_{a} \frac{\mu_{t}}{\sigma_{\varepsilon}} \nabla\left[\phi^{D} \varepsilon^{B}+\left(1-\phi^{D}\right) \varepsilon^{n}\right]_{a} \bullet A_{a} \\
& +\varepsilon^{n}
\end{aligned}
$$

\subsection{RSTM Solution Procedure}

The six components of the discretized Reynolds-stress transport equation, Eq. (4.21), have been decoupled due to linearization of the source terms. They are, however, still coupled in the azimuthal direction through the periodic boundary condition for cylindrical grids. Since Reynolds-stress is a second-order tensor, the following 
transformation matrix, as well as its transpose, are applied during the rotation of the Reynolds-stresses for periodic boundaries:

$$
\left[\begin{array}{ccc}
\cos \theta & \sin \theta & 0 \\
-\sin \theta & \cos \theta & 0 \\
0 & 0 & 1
\end{array}\right]
$$

where $\theta$ is the cylindrical sector angle. Due to the coupling through Eq. (4.45), the six Reynolds-stress equations, Eq. (4.21), are solved in Phase B (immediately after the pressure-velocity iteration) using the conjugate residual iterative method [O'Rourke and Amsden, 1986].

To further enhance the numerical stability of the solver, the diffusion term in the RANS equation, Eqs. (4.1) and (4.2), is re-written as [Basara, 1993]

$$
\frac{\partial \tau_{i j}}{\partial x_{j}}=\frac{\partial}{\partial x_{j}}\left(\mu \chi_{i j}\right)=\left[\frac{\partial}{\partial x_{j}}\left(\mu_{e} \chi_{i j}\right)\right]^{n+1}-\left[\frac{\partial}{\partial x_{j}}\left(\mu_{t} \chi_{i j}\right)\right]^{n}
$$

where

$$
\chi_{i j}=\frac{\partial U_{i}}{\partial x_{j}}+\frac{\partial U_{j}}{\partial x_{i}}-\frac{2}{3} \frac{\partial U_{k}}{\partial x_{k}} \delta_{i j}
$$

and $\mu_{e}$ is the effective viscosity, defined as the sum of molecular viscosity and eddy viscosity, i.e.,

$$
\mu_{e}=\mu+\mu_{t}
$$

The conjugate residual iterative method is now briefly described. The evaluations of the Reynolds-stresses and dissipation-rate are carried out immediately following the outer iteration described above. For convenience, only the solution procedure for 
dissipation rate, $\varepsilon$, will be given below. The Reynolds-stress equations are similarly handled.

Recalling Eq. (4.44), the discretized equation for $\varepsilon$,

$$
\begin{aligned}
\varepsilon^{B}= & -\left(\frac{2}{3} C_{\varepsilon 1}-C_{\varepsilon 3}\right)\left(\frac{1}{V}\right)^{B}\left\{\left(V^{B}-V^{n}\right)-\max \left[\left(V^{B}-V^{n}\right), 0.0\right]\right\} \varepsilon^{n} \\
& +\left[-\left(\frac{2}{3} C_{\varepsilon 1}-C_{\varepsilon 3}\right)\left(\frac{1}{V}\right)^{B}\left\{\max \left[\left(V^{B}-V^{n}\right), 0.0\right]\right\} \varepsilon^{B}\right] \\
& +\Delta t\left(\frac{\varepsilon}{k}\right)^{n}\left(-C_{\varepsilon 1} \wp^{n}\right) \\
& +\Delta t\left(\frac{\varepsilon}{k}\left(-C_{\varepsilon 2}\right)\right)^{n} \varepsilon^{B} \\
& +\frac{\Delta t}{M^{B}} \sum_{a} \frac{\mu_{t}}{\sigma_{\varepsilon}} \nabla\left[\phi^{D} \varepsilon^{B}+\left(1-\phi^{D}\right) \varepsilon^{n}\right]_{a} \bullet A_{a} \\
& +\varepsilon^{n}
\end{aligned}
$$

one can set an intermediate value of dissipation rate, $\overparen{\varepsilon}$, consisting of only the explicit terms (time level " $n$ ", or known quantities), as

$$
\begin{aligned}
\widehat{\varepsilon}= & -\left(\frac{2}{3} C_{\varepsilon 1}-C_{\varepsilon 3}\right)\left(\frac{1}{V}\right)^{B}\left\{\left(V^{B}-V^{n}\right)-\max \left[\left(V^{B}-V^{n}\right), 0.0\right]\right\} \varepsilon^{n} \\
& +\Delta t\left(\frac{\varepsilon}{k}\right)^{n}\left(-C_{\varepsilon 1} \wp^{n}\right) \\
& +\frac{\Delta t}{M^{B}} \sum_{a} \frac{\mu_{t}}{\sigma_{\varepsilon}} \nabla\left[\left(1-\phi^{D}\right) \varepsilon^{n}\right]_{a} \bullet \boldsymbol{A}_{a} \\
& +\varepsilon^{n}
\end{aligned}
$$

Substituting $\overparen{\varepsilon}$ into Eq. (4.49), 


$$
\begin{aligned}
\varepsilon^{B}= & \widehat{\varepsilon} \\
& +\left[-\left(\frac{2}{3} C_{\varepsilon 1}-C_{\varepsilon 3}\right)\left(\frac{1}{V}\right)^{B}\left\{\max \left[\left(V^{B}-V^{n}\right), 0.0\right]\right\} \varepsilon^{B}\right] \\
& +\Delta t\left(\frac{\varepsilon}{k}\left(-C_{\varepsilon 2}\right)\right)^{n} \varepsilon^{B} \\
& +\frac{\Delta t}{M^{B}} \sum_{a} \frac{\mu_{t}}{\sigma_{\varepsilon}} \nabla\left[\phi^{D} \varepsilon^{B}\right]_{a} \bullet A_{a}
\end{aligned}
$$

The conjugate residual iterative method requires an initial guess for $\varepsilon^{B}$, which is taken to be

$$
\varepsilon^{B}=\frac{\widehat{\varepsilon}}{R_{\varepsilon}}
$$

where

$$
R_{\varepsilon}=1-\left[-\left(\frac{2}{3} C_{\varepsilon 1}-C_{\varepsilon 3}\right)\left(\frac{1}{V}\right)^{B}\left\{\max \left[\left(V^{B}-V^{n}\right), 0.0\right]\right\}\right]-\Delta t\left(\frac{\varepsilon}{k}\left(-C_{\varepsilon 2}\right)\right)^{n}
$$

For a converged solution, one should obtain

$$
\varepsilon^{B} \times R_{\varepsilon}-\widehat{\varepsilon}-\frac{\Delta t}{M^{B}} \sum_{a} \frac{\mu_{t}}{\sigma_{\varepsilon}} \nabla\left[\phi^{D} \varepsilon^{B}\right]_{a} \bullet A_{a}=0
$$

although in practice, the right-hand-side will not be zero but instead, a small number called "residual." Accordingly, the residual of the dissipation-rate equation can be expressed as

$$
\operatorname{Res}\left(\varepsilon^{B}\right)=\varepsilon^{B} \times R_{\varepsilon}-\overparen{\varepsilon}-\frac{\Delta t}{M^{B}} \sum_{a} \frac{\mu_{t}}{\sigma_{\varepsilon}} \nabla\left[\phi^{D} \varepsilon^{B}\right]_{a} \bullet \boldsymbol{A}_{a}
$$

Furthermore, its gradient, i.e., the change of residual with respect to changes in the $\varepsilon$ guess, can subsequently be calculated:

$$
\frac{d}{d \varepsilon^{B}}\left[\operatorname{Res}\left(\varepsilon^{B}\right)\right]=R_{\varepsilon}-\frac{d}{d \varepsilon^{B}}\left\{\frac{\Delta t}{M^{B}} \sum_{a} \frac{\mu_{t}}{\sigma_{\varepsilon}} \nabla\left[\phi^{D} \varepsilon^{B}\right]_{a} \bullet A_{a}\right\}
$$


since $\widehat{\varepsilon}$ is a known quantity. Based on Jacobi preconditioning, the diagonal elements of this gradient is then used as the preconditioning matrix $\underset{\sim}{A}$ to speed up the convergence of the iterations, i.e.,

$$
\underset{\sim}{A}=1 /\left\{\frac{d}{d \varepsilon^{B}}\left[\operatorname{Res}\left(\varepsilon^{B}\right)\right]\right\}_{\text {diagonal elements }}
$$

Next, the change of the residual, $d[\operatorname{Res}(\varepsilon)]_{\text {guess }}$, is orthogonalized with respect to the previous direction of $d[\operatorname{Res}(\varepsilon)]$, according to

$$
d \varepsilon=-\underset{\sim}{A} \times \operatorname{Res}(\varepsilon)_{\text {previous }}-\lambda \times d \varepsilon_{\text {previous }}
$$

and

$$
d[\operatorname{Res}(\varepsilon)]=d[\operatorname{Res}(\varepsilon)]_{\mathrm{guess}}-\lambda \times d[\operatorname{Res}(\varepsilon)]_{\mathrm{previous}}
$$

where

$$
\lambda=\frac{\left[d[\operatorname{Res}(\varepsilon)]_{\text {guess }}, d[\operatorname{Res}(\varepsilon)]_{\text {previous }}\right]}{\left[d[\operatorname{Res}(\varepsilon)]_{\text {previous }}, d[\operatorname{Res}(\varepsilon)]_{\text {previous }}\right]}
$$

and [, ] is the inner product operator. The left hand side of Eq. (4.59) is the new direction vector.

The components of the new $d[\operatorname{Res}(\varepsilon)]$ and $d \varepsilon$ in the new direction are found, and finally added to the solution:

$$
\varepsilon^{B}=\varepsilon_{\text {previous }}+\underset{\sim}{A} \times \operatorname{Res}(\varepsilon)_{\text {previous }}+\alpha \times d \varepsilon
$$

where

$$
\alpha=\frac{\left[\operatorname{Res}(\varepsilon)_{\text {previous }}, d[\operatorname{Res}(\varepsilon)]\right]}{[d[\operatorname{Res}(\varepsilon)], d[\operatorname{Res}(\varepsilon)]]}
$$

The solution is considered converged if

$$
|\alpha \times d \varepsilon|<E \times \varepsilon^{B}
$$


where $E$ is the relative error allowed in the diffusion of $\varepsilon$, typically having a value of 0.001 .

A description of the major modifications to KIVA can be found in Appendix A.

\subsection{Validations}

Basic validations of the newly implemented Reynolds-stress turbulence model in KIVA are achieved through calculations of two problems: channel flow and pipe flow, both fully-developed. Computed results are compared and validated against experimental (pipe) and DNS (channel) data. Further comparisons between the simulation results and measurements in Chapter 5 provide additional validations for the RSTM.

\subsubsection{Pipe Flow}

The axisymmetric pipe flow data of Laufer [1952] is used. The pipe radius is $2.5 \mathrm{~cm}$. To accurately simulate a fully-developed flow, a long pipe should be used. However, it is computationally expensive as well as redundant to perform the actual flow development. A strategy is the use of a short length of the pipe, applying periodic boundary condition with volumetric flow rate correction on both the inflow and outflow boundaries until the flow has fully developed. The pipe length is chosen to be $3 \mathrm{~cm}$ for the computations.

A two-dimensional grid is used, with periodic boundary condition applied in the azimuthal direction. Figure 4.7 shows the computational mesh and its orientation. The coordinate system in the plots presented below has been transformed from Cartesian (as in KIVA) to cylindrical, with " $\mathrm{x}$ " representing the axial direction, and " $\mathrm{r}$ " radial. The grid 
contains 40 cells in the radial direction, and 30 axially. A two-sided stretching function [Hoffmann and Chiang, 1993] is utilized to cluster the grid near the wall and centerline for better resolution of the flow.

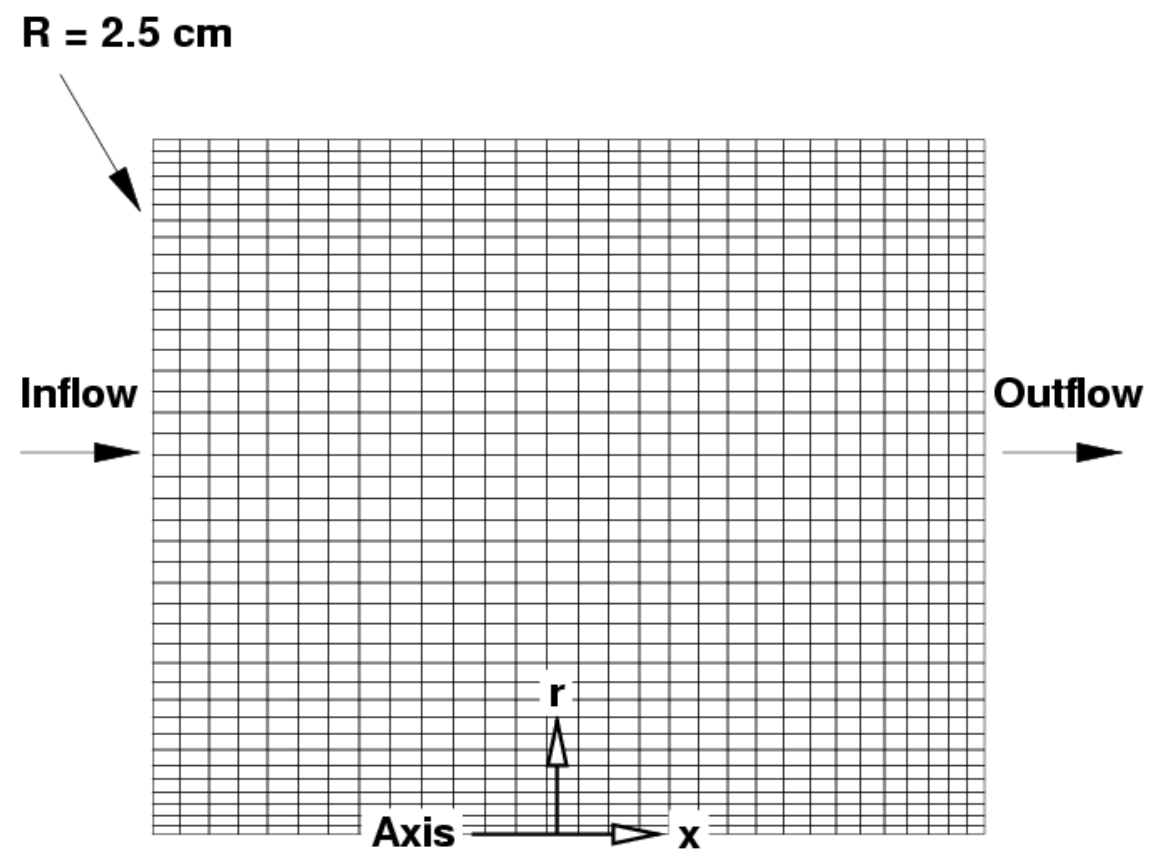

Figure 4.7. Computational Mesh of the Pipe

The fluid is air under standard conditions, i.e., at a temperature of $300 \mathrm{~K}$ and pressure of one atmosphere. Initial velocity profile at the inlet is uniform, with a magnitude of $13.47 \mathrm{~m} / \mathrm{s}$. Accordingly, the Reynolds number based on the pipe diameter, $R e_{\mathrm{d}}$, is 40,000 .

Figure 4.8 presents the result comparisons. The Reynolds-stress data agree well with the measurements, as well as the profile of turbulent boundary layer. 

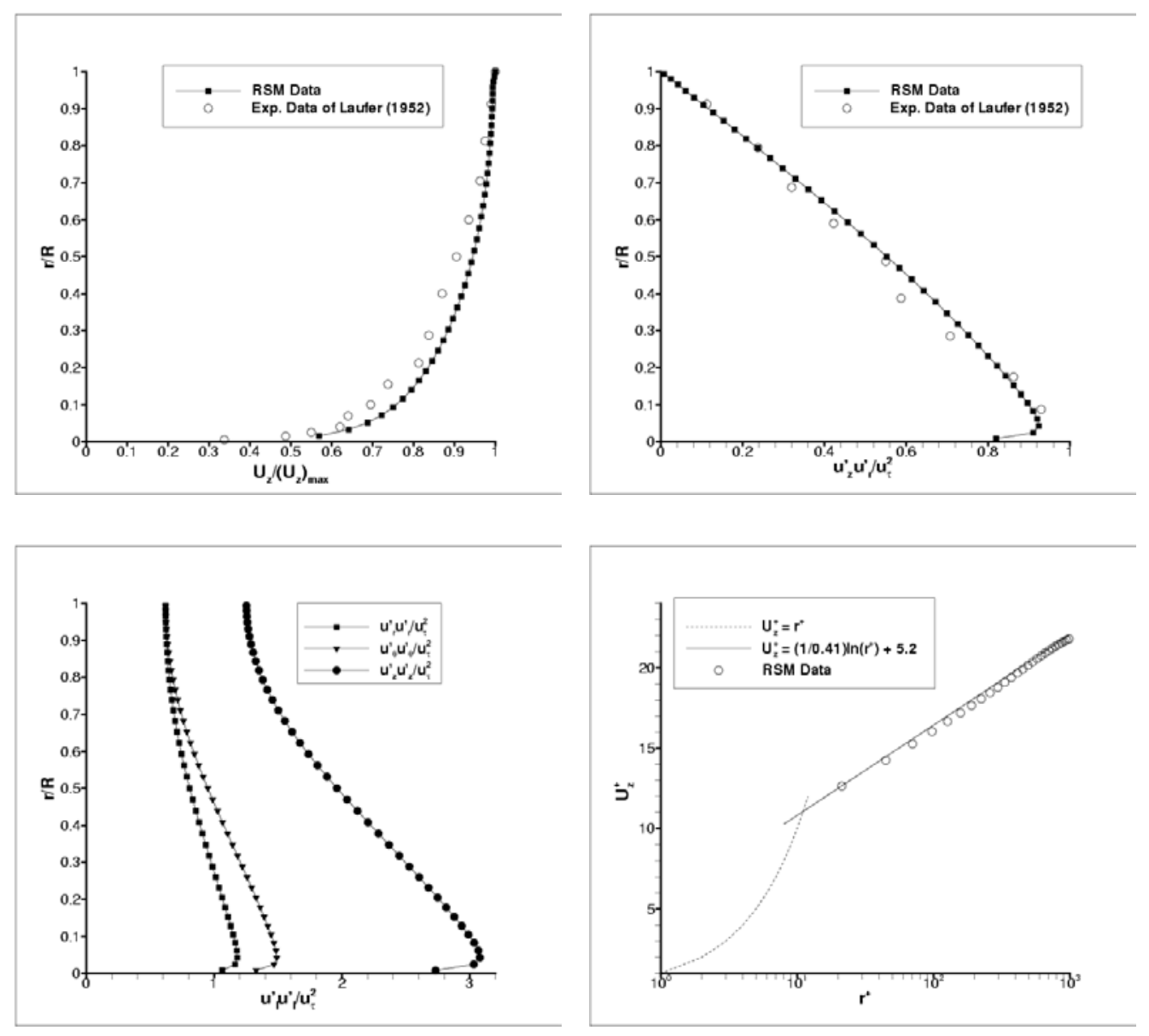

Figure 4.8. Pipe Flow Result Comparisons Between RSTM and Measurements

\subsubsection{Channel Flow}

Validation for the RSTM is further accomplished by comparing results for flow through a channel. DNS data of Mansour et al. [1988] is used for quantitative comparisons. The channel under consideration is $5 \mathrm{~cm}$ wide and $1 \mathrm{~cm}$ long. Such short length is the result of the same computational strategy described in the pipe-flow section above. 
The computational grid, shown in Figure 4.9 below, has 50 and 10 cells uniformly spread across the width and length, respectively. A denser grid of 100-by-20 cells is also used, and the computed results are nearly identical to the current grid.

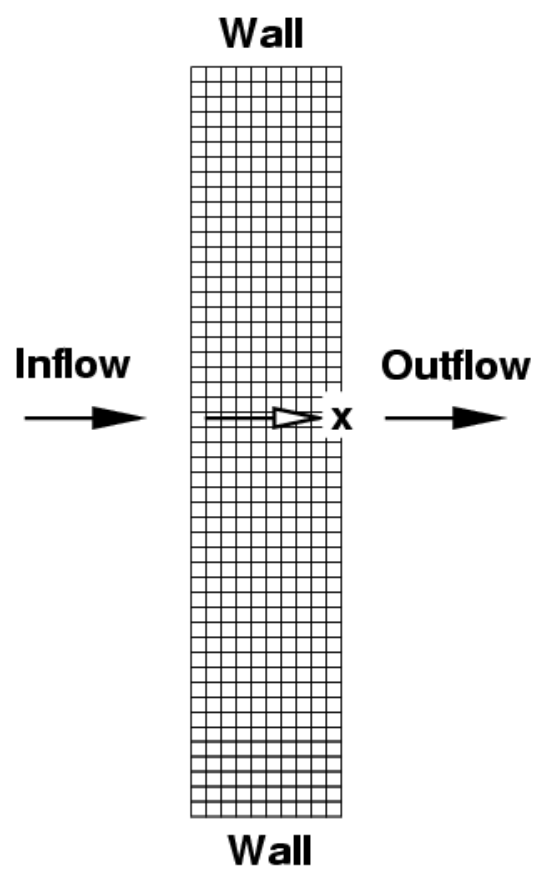

Figure 4.9. Computational Mesh of the Channel

Air is used as the medium, at a temperature of $300 \mathrm{~K}$ and $1 \mathrm{~atm}$. A uniform velocity of $4.6316 \mathrm{~m} / \mathrm{s}$ is specified at the inflow boundary. The Reynolds number based on the channel height, $R e_{\mathrm{H}}$, is 13,750 .

The comparisons between the RSTM result and DNS data, presented in Figure. 4.10, prove the validity and accuracy of the turbulence model. 

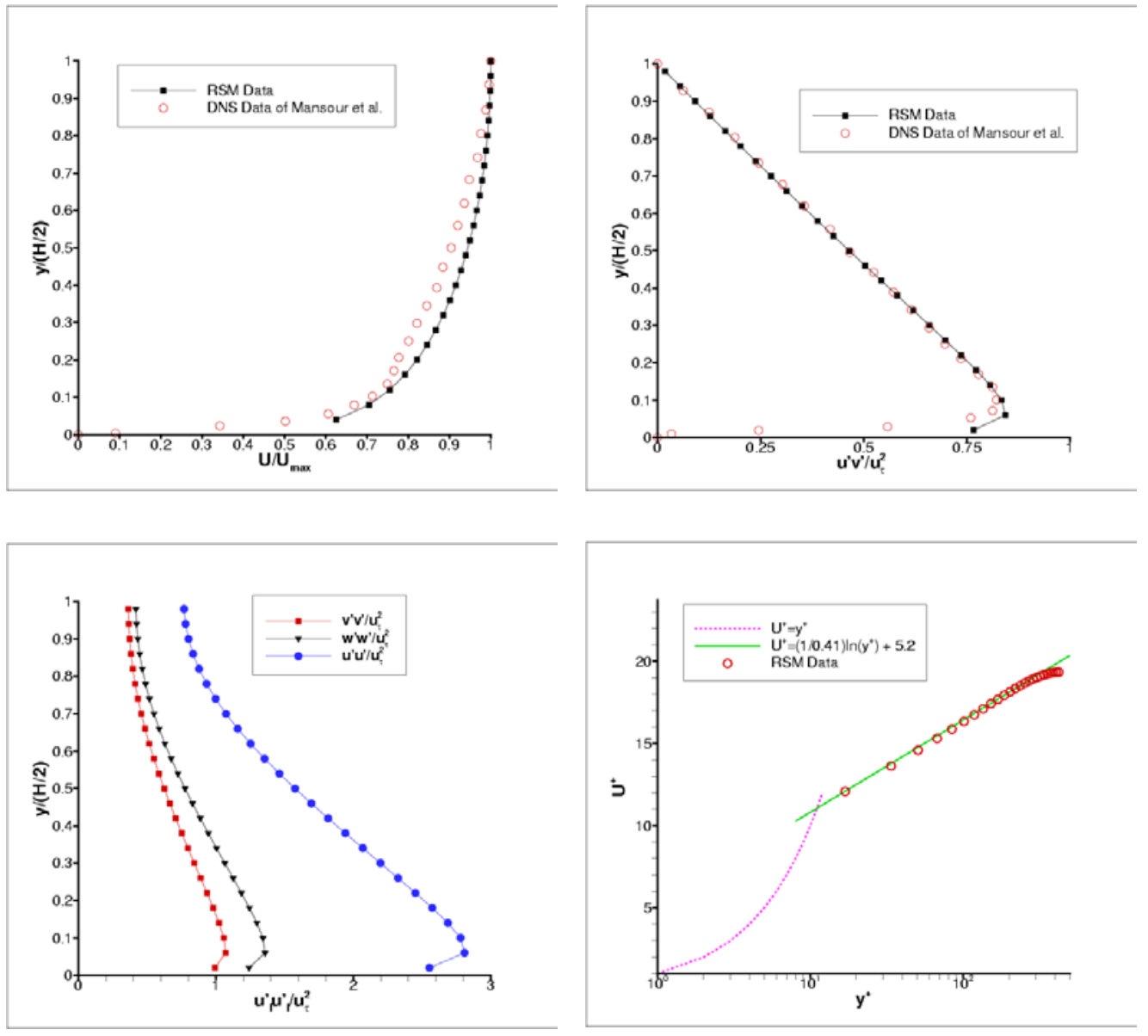

Figure 4.10. Channel Flow Result Comparisons Between RSTM and DNS Data 


\section{Chapter 5}

\section{Applications}

The KIVA-3V Release 2 code equipped with Reynolds-stress turbulence models has been applied to a number of gas turbine engine designs. The geometry of each engine under investigation includes air swirlers, forward-facing steps (sudden expansion), a convergent section, and/or fuel injectors. Therefore, the flow fields are relatively complex, and strong swirl, flow separation, recirculation, and other flow phenomena are expected to be present.

In each of the cases to be presented, the computed results using Reynolds-stress turbulence model are compared with experimental data wherever available. Calculations performed with other turbulence models, e.g., the standard $k-\varepsilon$ model, are also presented to provide a reference for the improvement of accuracy achieved by using the RSTM. 


\subsection{Lean-Direct-Injection (LDI) Combustor with Axial Swirlers}

This study involves recent research on the use of a Reynolds-stress turbulence model (RSTM) for three-dimensional flow field simulation inside the gas turbine combustors. It intends to show the motivations for using the RSTM in engine flow simulation, to present a further validation of the RSTM implementation in the KIVA code using the available experimental data, and to provide comparisons between the RSTM and $k$ - $\varepsilon$ turbulence model results for chemically nonreacting swirling flows. The results show that, for high-degree swirl flow, the RSTM can provide predictions in favorable agreement with the experimental data, and that the RSTM predicts recirculations and high velocity gradients better than does the $k-\varepsilon$ turbulence model. The results also indicate that the choice of swirler design has a significant influence on the structure of the combustor flow field.

\subsubsection{Introduction}

A key characteristic of gas turbine combustor performance is the emission of $\mathrm{NO}_{\mathrm{x}}$. Although the emissions of unburned hydrocarbons and carbon monoxide have been greatly reduced through the design process, the problem of $\mathrm{NO}_{\mathrm{x}}$ emission still requires significant improvements in combustor design [Koff, 1994].

Combustion at near stoichiometric fuel-air ratios produces high combustion temperatures which are the main cause of high thermal $\mathrm{NO}_{\mathrm{x}}$ emissions. As a result, combustor designs have sought nonstoichiometric fuel-air ratios in both rich and lean ranges. Rich burn combustion reduces $\mathrm{NO}_{\mathrm{x}}$ by limiting the amount of oxygen present for high temperature combustion. Combustors like these are often labeled rich-burn quick- 
quench lean-burn (RQL). Here, a rich burn primary zone is followed by a quick quench zone where the combustion gases are diluted before combustion is completed in a cooler lean burn zone. This design concept has been the subject of several studies, [Talpallikar et al., 1992; Cline et al., 1995; Yang et al., 1992; Micklow et al., 1993]. An alternative to RQL is lean-premixed combustion in which a lean combination of fuel and air is premixed and prevaporized prior to introduction into the combustor. It has also been studied by several researchers [Kumakura et al., 1993; Cowell and Smith, 1993; Leonard and Stegmaier, 1994], and it was found that low $\mathrm{NO}_{\mathrm{x}}$ emissions required uniform fuel-air mixtures. Tacina [1990] found that a lean premixed combustor produced less $\mathrm{NO}_{\mathrm{x}}$ in comparison with the RQL.

A third approach is called lean direct injection (LDI). Instead of injecting a lean premixed quantity of fuel and air into the combustor, fuel is injected into the airflow entering the combustor. Such a combustor depends on fuel injectors which can produce a spray of very fine droplets that quickly atomize and vaporize. Furthermore, the mixing process is also a key issue for the LDI.

Several studies have pursued computational fluid dynamics techniques to predict the flowfield inside the combustor. Successful predictions of turbulent reactive flows depend highly on successful prediction of the turbulent fluid flow [Gran et al., 1997]. A popular choice for the turbulence model has been the two-equation $k$ - $\varepsilon$ eddy viscosity turbulence model (EVTM). Such calculations have been performed, [Cline et al., 1995; McGuirk and Palma, 1993; Rizk and Mongia, 1993; Relation et al., 1998]. Despite its popularity and low computational overhead, the standard $k-\varepsilon$ model suffers from several deficiencies. It is known for being inappropriate for modeling swirling flows [Sloan et al., 
1986], which are often present and desired in combustors and IC engines. More often than not, ad hoc modifications to the $k-\varepsilon$ equations are necessary to provide satisfactory results. It also tends to incorrectly predict recirculation regions [Gran et al., 1997]. Prediction of these flow structures is important since the location of recirculation regions can influence the residence time involved in $\mathrm{NO}_{\mathrm{x}}$ production [Micklow et al., 1993], and/or can increase the residence time for liquid fuel evaporation. Finally, the standard $k-\varepsilon$ model often underpredicts large velocity gradients because of the diffusive nature of this model [Relation et al., 1998].

In contrast, the Reynolds stress turbulence model (RSTM) provides hope for better accuracy in predicting the complex swirling flow, which may also contain recirculation, while maintaining an economical computational overhead for modern workstations and personal-computer clusters. Unlike the standard EVTM, the RSTM is able to capture the anisotropy caused by complex flow, flow rotation (swirl flow), streamline curvature, wall effects, etc. This is achieved naturally since each Reynolds-stress component is solved by its own transport equation. As a result, the RSTM is needed to properly address these flow issues.

Re-stress calculations have been performed on combustors [Tsao and Lin, 1999; Lin and Lu, 1994], for cases with low and high inlet swirl. In each study the Gibson and Launder [1978], RSTM was used with a modified turbulence specific dissipation $(\varepsilon)$ equation. Meanwhile, promising results were observed in an RSTM study by Yang et al. [2000] when applying the Speziale-Sarkar-Gatski (SSG) model of Speziale et al. [1991] to reciprocating engines and to a lean premixed combustor. 
The purpose of this study is to expound upon the report by Yang et al. [2000] by further investigating and validating the SSG RSTM for nonreacting swirl flow calculation of an LDI combustor using the KIVA-3V computer code [Amsden, 1997]. The study is to substantiate the RSTM as a preferred turbulence model by presenting evaluations and comparisons of the results with available experimental data and $k$ - $\varepsilon$ turbulence model results. It is also desired to show that, unlike eddy-viscosity turbulence models, an RSTM does not require ad hoc modification, while being capable of capturing the complex characteristics of turbulent swirling flow.

\subsubsection{RSTM and Code Implementation}

The detail of the RSTM has been given in Chapter 3 and 4 of this dissertation. Additionly, in Yang et al. [2000], the performance of the SSG RSTM for simulating turbulent flow inside the cylinder of IC engines and combustor was reported. One major advantage of using the SSG model is that, unlike most other RSTMs available in the literature, no wall-distance parameter is needed. Because of the complex geometry involved, this feature is especially desirable and important for engine flow simulation. Code implementation and validation can be found in Chapter 4. In this study, additional model validation and performance of the SSG model will be given below.

\subsubsection{Description of the Problem}

For gas turbine combustor, swirl flow is used to improve combustor performance by aiding in the fuel-air mixing process and by producing recirculation regions which can act as flame holders for flame stability. As mentioned earlier, recirculation can influence 
residence time and, as a result, NOx production. Therefore, proper selection of a swirler is needed to reduce $\mathrm{NO}_{\mathrm{x}}$.

In this study, an LDI combustor is considered. The modeled LDI combustor includes inlets at nine inflow locations, which are attached to a single primary combustion zone shown in Figure 5.1.1. The inlets are atop helical axial swirlers arranged in a threeby-three square which lies in the xy-plane. The rotation imparted to the air by each swirler is directed in the axial or z-direction. Two different swirlers were used based on blade angle inclination from the direction of airflow. One swirler had a $45^{\circ}$ angle, and the other $60^{\circ}$. These angles refer to the outermost vane twisting angle of the helical swirler. A plot of a $60^{\circ}$ swirler attached to a convergent-divergent venturi is shown in Figure 5.1.2. The central hollow region is reserved for the fuel injector while the five helical hollow slots represent the blades that guide the airflow.

The computational domain begins with the inlets placed at the top of nine $45^{\circ}$ or $60^{\circ}$ swirlers. These swirlers are either co-rotating clockwise, or counter-rotating. After air exits a swirler it passes through a venturi. At the center of the venturi circle, a fuel injector nozzle can inject liquid fuel. This arrangement is used since the combination of swirler and venturi were found to have a significant influence on the liquid atomization and droplet distribution [Im et al., 1998]. A short divergent cone, acting like a diffuser, follows and ends at the primary zone inlet plane. Two constant-y planes and three constant-z planes were defined for postprocessing, where $\mathrm{z}$ represents the axial direction. These cutting plane locations are shown in Figure 5.1.1.

Notable boundary conditions include the wall and inlet boundaries. Since the turbulence equations presented are valid only for high-Re number flows, wall functions 
were used for wall boundaries. Diffusion fluxes were set to zero at the walls. The specified inlet velocity value depends on the chosen operating conditions of the combustors. These conditions include a pressure drop, dp, of $3 \%$ across the height of the combustor, an outlet pressure of $1.0 \mathrm{~atm}$, and an inlet temperature of $27^{\circ} \mathrm{C}$. These quantities were then used to determine the inlet air velocity Vin, which is uniform at all nine swirlers, according to the following equation:

$$
V_{i n}=C_{D} \sqrt{\frac{2(\Delta p)}{\rho}}
$$

The discharge coefficient $C_{D}$ was experimentally determined and provided by the sponsor. Uniform inlet turbulence quantities of $k_{\text {in }}, \varepsilon_{\text {in }}$, and Reynolds stresses are listed in Table 5.1.

Three computational meshes with different grid densities, namely 113793, 173829, and 254853 grid points were used. Results from the three grids are consistent with each other. The medium density grid was used in the following presentations to represent the results of all three grids.

\subsubsection{Results and Discussions}

The purpose of this study was to investigate the nonreacting swirl flow within the combustor. Therefore, the spray and ignition options were turned off. Calculations were performed with the SSG RSTM and the $k$ - $\varepsilon$ model using the quasi-second order upwind scheme in the KIVA code. The computed results were compared and validated with the experimental data for the $60^{\circ}$ co-rotating case. In addition, a $45^{\circ}$ co-rotating and a $60^{\circ}$

counter-rotating-at-center case were also investigated. Since the computational domain 
was three-dimensional, results of these predictions are presented at the cutting planes indicated in Figure 5.1.1.

\section{Experiment}

The experiments were performed at the NASA Glenn Research Center (GRC)

CE 5. A square duct of quartz wall was used as the primary combustion zone for optical access. The sides of the square were $76.2 \mathrm{~mm}$ wide. A phase/Doppler particle analyzer was used for all velocity measurements. A two-component system using green $(514.5 \mathrm{~nm})$ and blue $(488.0 \mathrm{~nm})$ beams from an argon-ion laser operating at $1.5 \mathrm{~W}$ power output was used for the measurements. The transmitting optics utilized a $500 \mathrm{~mm}$ focal length lens combined with a $300 \mathrm{~mm}$ focal length collimating lens to yield a focused beam waist of $131 \mathrm{~mm}$ for the green and $124 \mathrm{~mm}$ for the blue lines. The fringe spacing was $6.788 \mathrm{~mm}$ for the green and $6.667 \mathrm{~mm}$ for the blue lines. The receiving optics were located 30 degrees off-axis in the forward-scatter direction. Light was collected using a $500 \mathrm{~mm}$ focal-length f5.4 lens and then focused onto a $100 \mathrm{~mm}$ by $1 \mathrm{~mm}$ long slit. Further details of the instrument can be found in Bachalo and Houser [1984], and details of the experimental procedure can be found in Bulzan [1995]. The experimental results are presented in Figures 5.1.3c-5.1.5c and are discussed below.

\section{$\underline{60^{\circ} \text { Co-rotating Swirlers Case }}$}

Observations of the results on the xy-planes show that the RSTM compares more closely to the experiments than does the $k$ - $\varepsilon$ model. The plots in Figures 5.1.3-5.1.5 display computational results across xy-planes, at $z=5 \mathrm{~mm}, 11 \mathrm{~mm}$, and $18 \mathrm{~mm}$ respectively, while the experimental plots show the data measurements at the upper right corner of the same xy planes. A coordinate system is displayed on the figures to indicate 
the $\mathrm{x}$ and $\mathrm{y}$ directions. The positive $\mathrm{z}$ direction, from the swirler inlet to the combustor outlet, points into the page.

Figure 5.1.3 paints a distinct picture that demonstrates the superiority of the RSTM when comparing its result with the $k-\varepsilon$ and experimental plots. Between the two turbulence models, the magnitude of the velocities differ greatly, although the axial velocity (w) contour structures and the vector orientations display similarities. Comparison with experimental data in Figure 5.1.3 shows that the reversed flow at the center of the circles is better captured by the RSTM. The contour legend shows that the RSTM velocity levels vary over a greater range that is closer to the experimental data plot, Figure 3a.

At $\mathrm{z}=11 \mathrm{~mm}$ in Figure 5.1.4, the RSTM continues to exhibit its capability as validated by the experiment. Unlike the $k-\varepsilon$ model result where no recirculation can be observed, the RSTM plot clearly shows a reversed flow region at each swirler center. Similar observations are found further downstream at the $\mathrm{z}=18 \mathrm{~mm}$ surface in Figure 5.1.5. The RSTM result shows that the forward flow between swirlers and reversed velocity magnitude at the swirler centers are in good agreement with the experiment. This shows that the RSTM can better predict the reversed flow associated with recirculations. It also shows the RSTM's natural ability to resolve the effects of strong swirl as verified by the experimental data.

Additional observations can be made from Figures 5.1.3-5.1.5. The four subregions enveloped by any four neighboring circles contain negative axial velocities (i.e., reversed flow) in the RSTM results, whereas the $k$ - $\varepsilon$ plots present little (Figures 5.1.3, 5.1.4) and no (Figure 5.1.5) reversed flow in these regions. Furthermore, these four sub- 
areas contain swirls in the counter-clockwise (ccw) direction, opposite to the primary flow direction from the nine swirlers. This is due to the opposing velocities surrounding the central circle, where the shear force in one direction impedes and steers away the shear force in the other, creating the four counter-clockwise swirls.

The contrast between the two models can be further displayed by viewing the velocity on the xz-planes, such as along the y0-y0 cutting plane shown in Figure 5.1.6. Reversed flow is much stronger below the swirlers with the RSTM. For example, the RSTM reverse flow reaches up to the venturi while the $k$ - $\varepsilon$ result falls far short. Concurrently, the forward flow regions predicted by the RSTM are also stronger. Since much of the cross section of the combustor has a reversed mass flux, the forward mass flux regions must be stronger to satisfy overall mass conservation.

Differences between the turbulence models can also be shown on the y1-yl cutting planes, which do not cut through the swirlers. According to Figure 5.1.7, reversed flow exists on this plane. The RSTM result shows two deep recirculation regions on the plane, while the $k-\varepsilon$ model in Figure 5.1.7 does not predict any reversed flow at all.

The $k-\varepsilon$ model results appear more diffusive than those of the RSTM. This is due to the overestimated eddy viscosity which is a known characteristic of the standard $k-\varepsilon$ model. This set of results also indicates that without ad hoc modification, the $k$ - $\varepsilon$ model is simply unable to capture the essence of flow recirculation from locally large axial pressure gradients, dp/dz, created by the high degree of swirl [Gupta et al., 1984]. As air exits from a swirler into the combustor, its swirling motion creates strong pressure gradients in the radial direction, a phenomenon similar to a free swirling jet being ejected from a nozzle. As the flow carries itself further downstream, an adverse pressure gradient builds up 
within the boundary of the air stream, causing the flow to reverse its axial direction and hence, a recirculation zone is created downstream of each swirler.

In short, with the RSTM whose Re-stress components are each computed with its own transport equation, the swirling and complex flow field can be resolved to an extent agreeable to the experiment. It can therefore be concluded that the RSTM has been correctly implemented, and that it is a valid model for the additional result comparisons discussed below.

\section{$\underline{45^{\circ} \text { Swirlers Case Study }}$}

The results obtained with the $45^{\circ}$ swirler primarily display the significant difference between swirlers. The magnitude of the inlet velocity is the same as in the $60^{\circ}$ swirler case. As Figures 5.1.8 and 5.1.9 indicate, there is no reversed flow at the center of the circles. Since the swirler diverts the upstream air at a smaller angle, a strong and solid forward-flow jet enters the combustor and does not permit recirculation as did the more conical jet from the $60^{\circ}$ swirlers. Due to the lower degree of swirl, both the turbulence models produce results almost identical to each other; however, weak recirculation regions are captured by the RSTM. Since the $45^{\circ}$ swirler produces such weak swirl flow, these results show that both the RSTM and $k-\varepsilon$ model are capable of predicting the flow field equally well.

The velocity profiles in Figure 5.1.9 suggest that the atomization, vaporization, and mixing processes would occur less effectively with the $45^{\circ}$ swirler. Fuel and air are injected directly downward into the primary zone, reducing the time for the three processes. Fuel-air jets around the perimeter are directed toward the wall and could lead to fuel droplet impingement on the walls. The lack of recirculation could reduce the amount 
of shear that enhances fuel atomization and the degree of mixing that leads to uniform lean combustion.

\section{$\underline{60^{\circ} \mathrm{cw}+\mathrm{ccw} \text { Swirlers Case Study }}$}

Comparisons are also made for $60^{\circ}$ swirlers where the center swirler twists in the counter- clockwise direction, while the outer eight remain clockwise. The results show a quite distinctive swirling profile compared to the two swirler arrangements presented above. The counter-rotation causes much higher velocity in the combustor around the central circle, as the xy-plane plots show in Figure 5.1.10. These two plots also show that the RSTM consistently predicts a more intense and vigorous velocity profile in comparison to the $k-\varepsilon$ model. The reversed twisting of the central swirler also brings about stronger and deeper recirculations, which are evident when comparing Figure 5.1.6 with Figure 5.1.11 where reversed flow can still be observed below the $\mathrm{z}=18 \mathrm{~mm}$ plane.

An apparent outcome of this arrangement is that reversing the central swirler rotation enhances the strength of swirl flow, not only in the vicinity of the central circle, but also throughout the entire region inside the combustor. This can be explained by carefully studying the nature of the interactions among the swirling flows emanated from the nine swirlers. In the vicinity where the central circle meets its neighboring four, velocity is enhanced and the swirl gains strength, since the flows are in the same directions in these regions. Unlike the co-rotating swirler configuration discussed above where "shear canceling" occurs surrounding the central circle, this $\mathrm{cw}+\mathrm{ccw}$ arrangement produces a more vigorous swirling flow under the same inlet boundary conditions and combustor geometry. 
It is also noted that, with this swirler arrangement, the recirculations exist at different locations from those of the co-rotating configuration. A comparison between the y0-y0 cutting planes in Figure 5.1.6 and Figure 5.1.11 indicates that the reversed flow exhibits different patterns between the arrangements. Figure 5.1.6 shows four recirculation regions, all of which are located near the junctions of the swirlers and away from the vertical combustor walls. In Figure 5.1.11, however, the four recirculation zones exist further away from one another. In particular, the two outer recirculations reside nearer the combustor walls. Such dissimilarity in the spatial locations of the recirculation regions may lead to different combustor designs, in terms of thermal protection on the combustor walls, flame stability, and pollutant emission control. These issues will be considered and addressed when flows with chemistry are studied.

\subsubsection{Conclusion}

The performance of the SSG Reynolds Stress Closure model using the KIVA-3V computer code is presented for chemically nonreacting flow simulations inside an LDI combustor. The RSTM performed better than the standard $k-\varepsilon$ model when comparisons were made with experiment for $60^{\circ}$ co-rotating swirlers. The primary improvements were the capturing of recirculation regions and the accompanying reversed flow. These flow characteristics were predicted to be stronger and more distinct with the RSTM. The results reaffirm a previous research statement that the RSTM can out-perform the $k$ - $\varepsilon$ model when swirling flows and recirculations are present. The results from the $45^{\circ}$ swirler type indicated that the choice of swirler also plays an important role in the production of swirl and recirculation. The results from the $60^{\circ}$ swirlers with reversed twisting angle at the 
center were also studied. This set of results clearly demonstrated the advantage the RSTM has over the $k-\varepsilon$ model for parametric studies. Since in the RSTM each component of the Re-stresses is computed individually, no ad hoc modification is required when the problem geometry or flow condition is altered. In addition, the computation time for the RSTM increased only slightly in comparison to the $k-\varepsilon$ model. The $k-\varepsilon$ model required 0.5039 msec/cycle/grid point, whereas the RSTM required $0.5649 \mathrm{msec} / \mathrm{cycle} /$ grid point. In summary, RSTM has become a favorable method for providing predictions of turbulent swirling flow within a combustor. Success in these aspects should aid in providing successful predictions when chemically reactive flows are studied.

Quantitative comparisons showing the improvement experienced with the RSTM can be found in Yang et al. [2003b] and Teo et al. [2001], which describe simulation results for an LDI combustor with discrete-jet swirlers using the RSTM. The comparisons were made to LDV data [Jeng et al., 2001] as well as results from the National Combustor Code using a cubic nonlinear $k-\varepsilon$ model [Iannetti et al., 2001a] tuned for swirl flow calculations. 


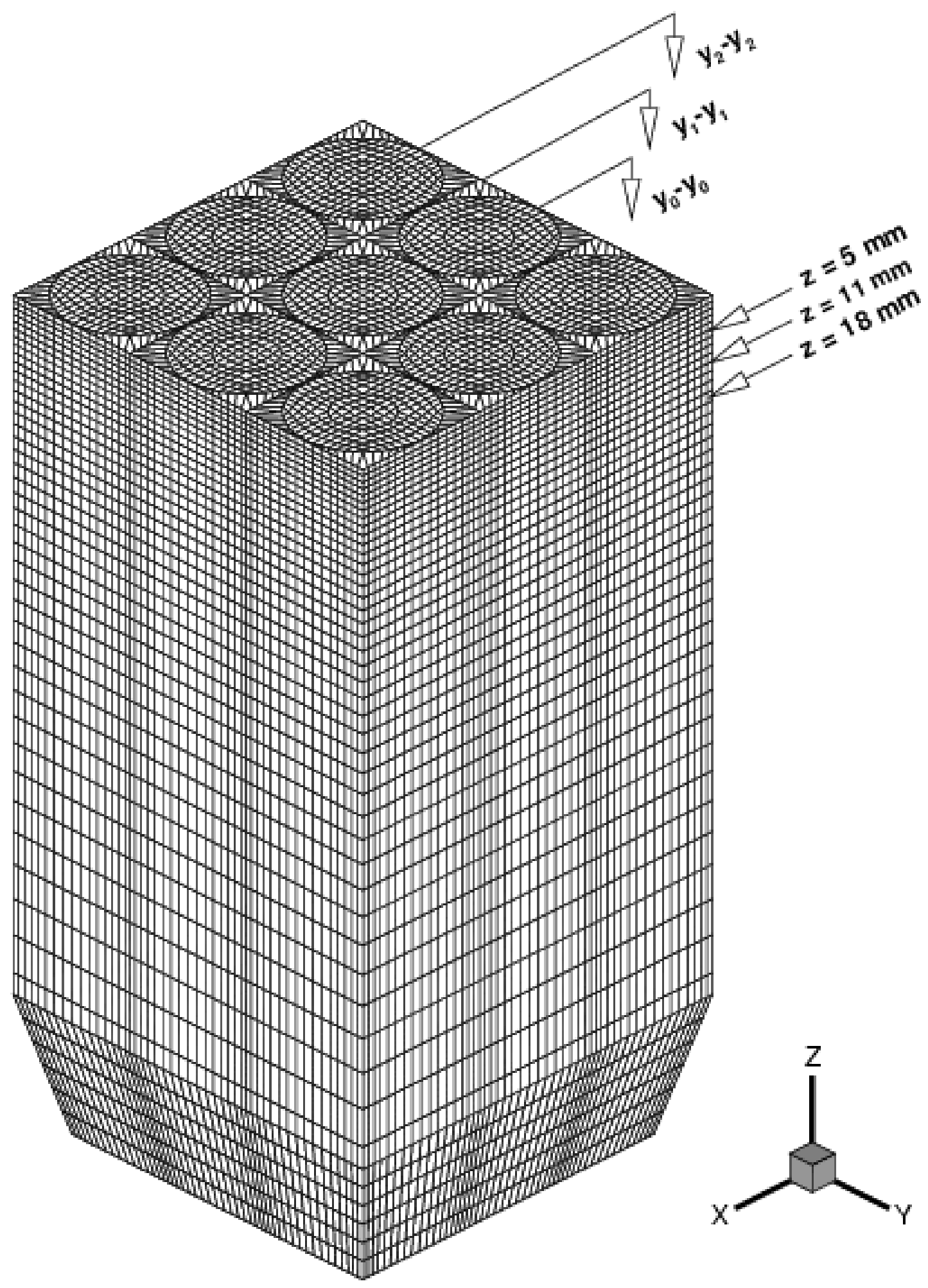

Figure 5.1.1. Combustor Mesh and Cutting Planes 


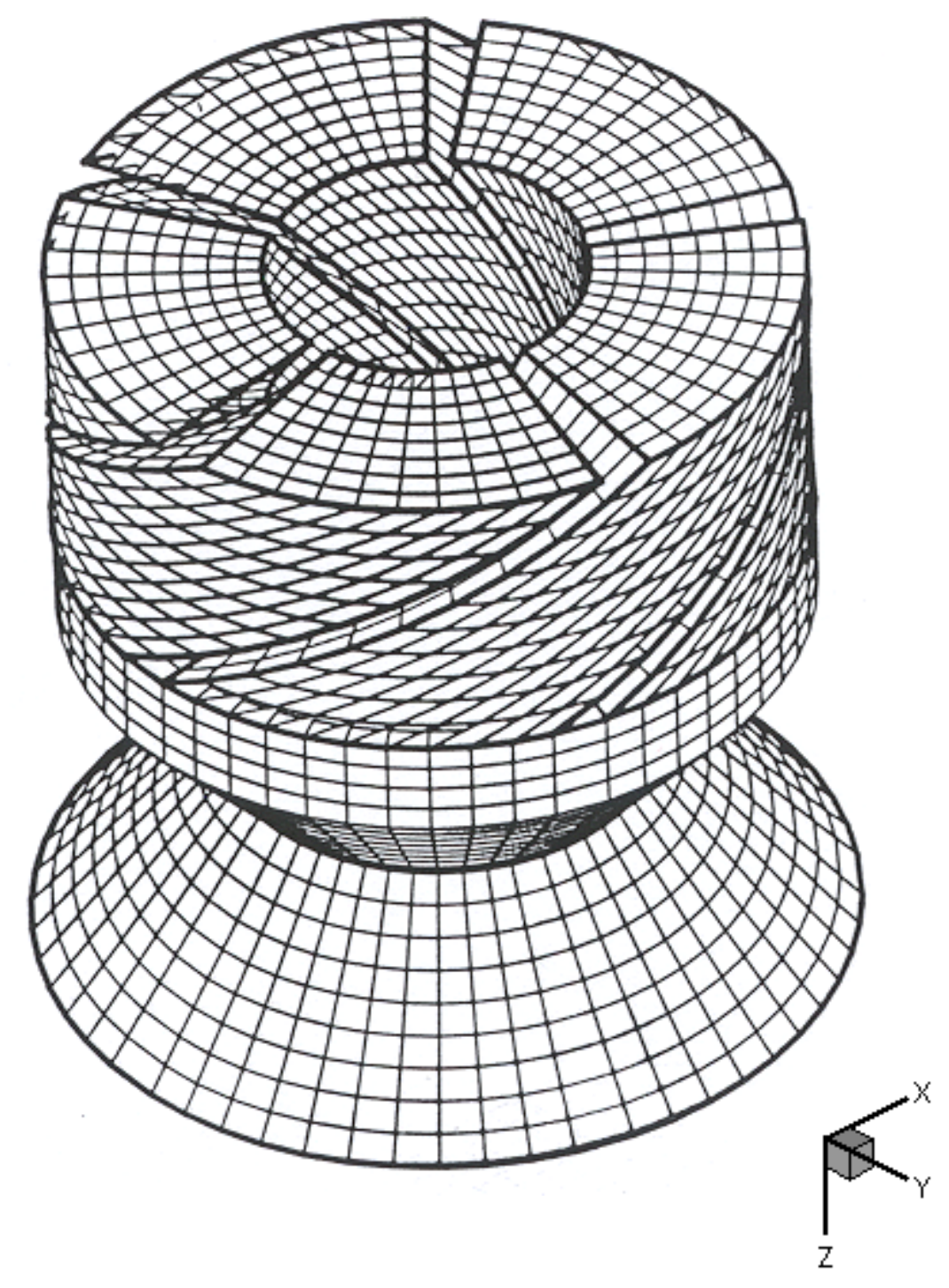

Figure 5.1.2. A Sketch of the Swirler 


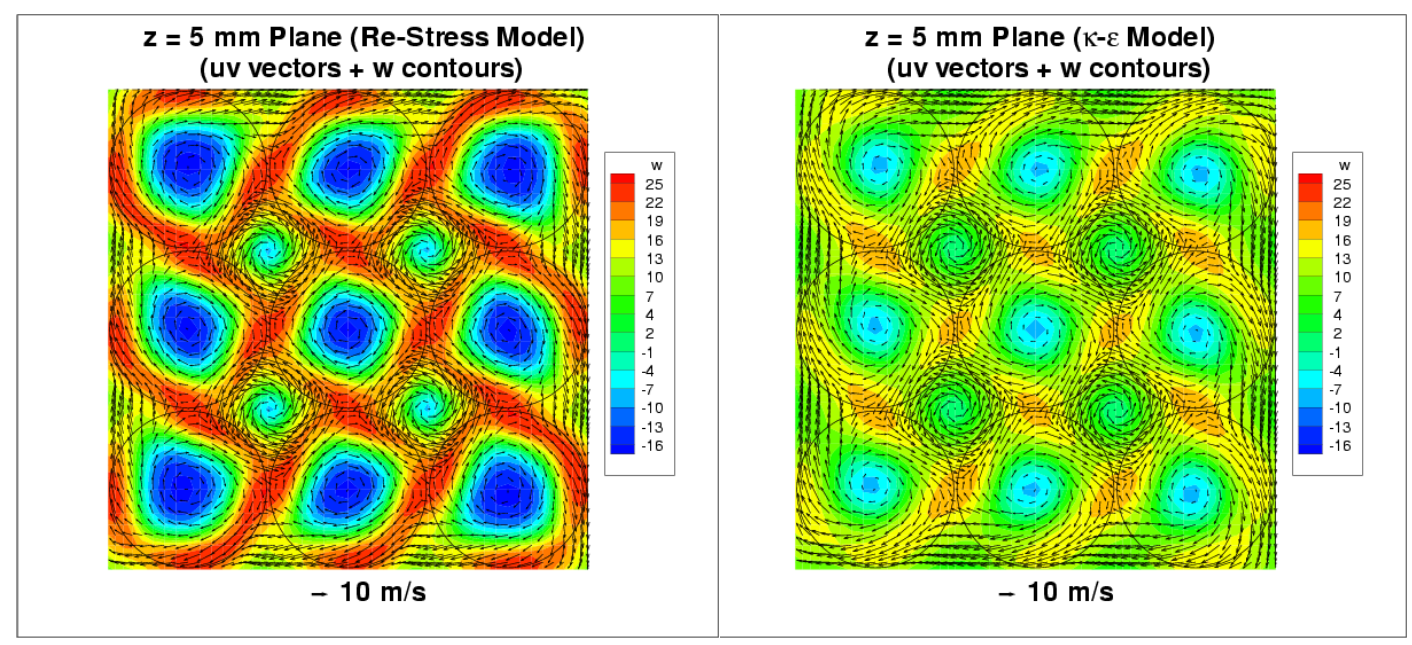

(a) RSTM

(b) $k-\varepsilon$

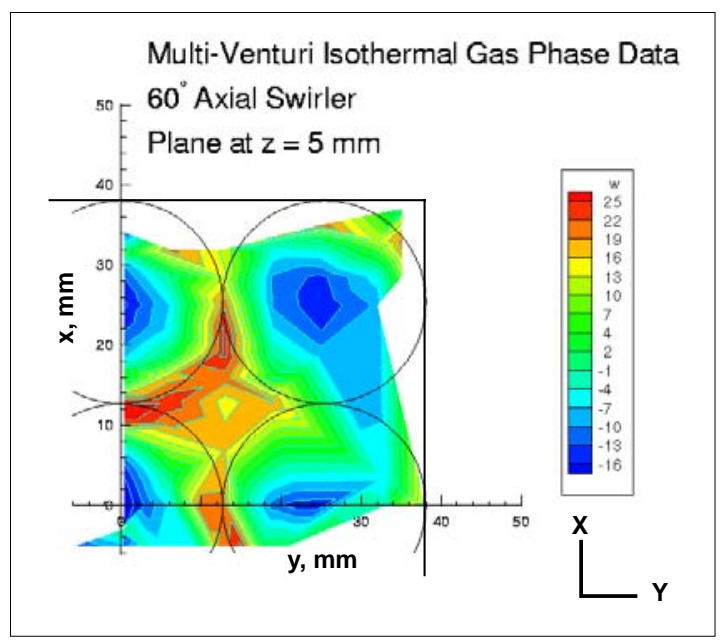

(c) Measurements

Figure 5.1.3. Results for $60^{\circ}$ Swirler at $\mathrm{Z}=5 \mathrm{~mm}$ Plane 


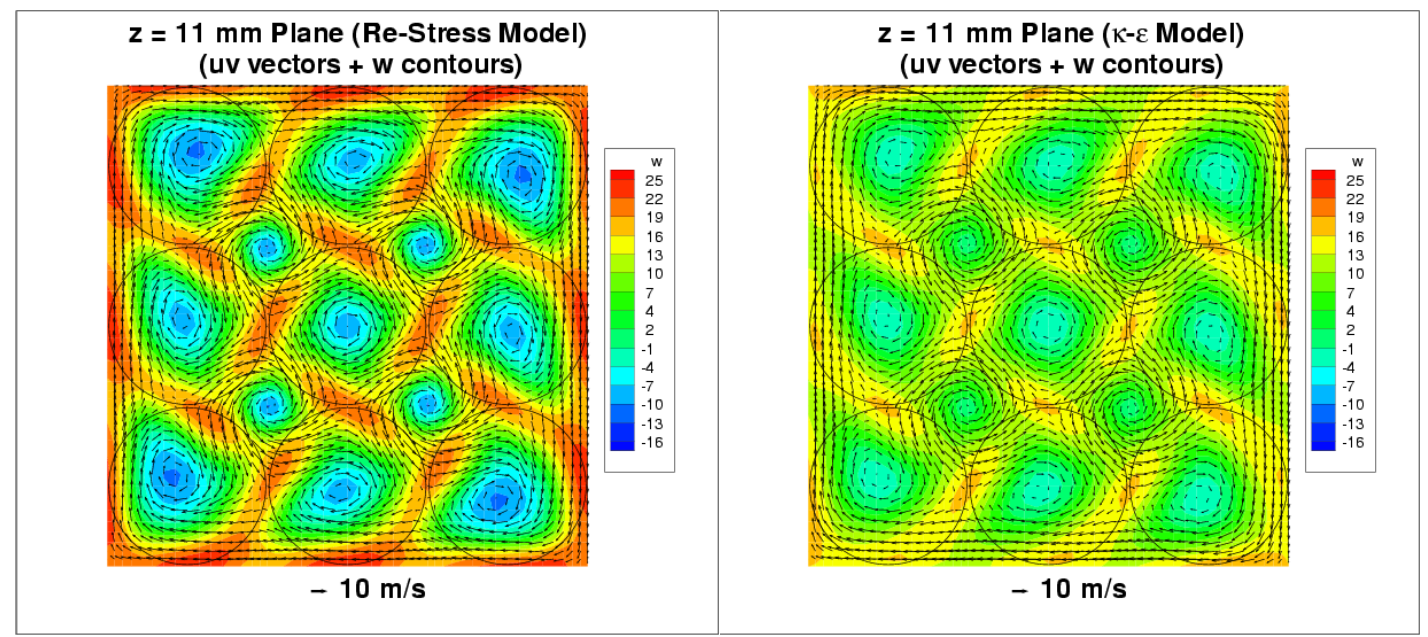

(a) RSTM

(b) $k-\varepsilon$

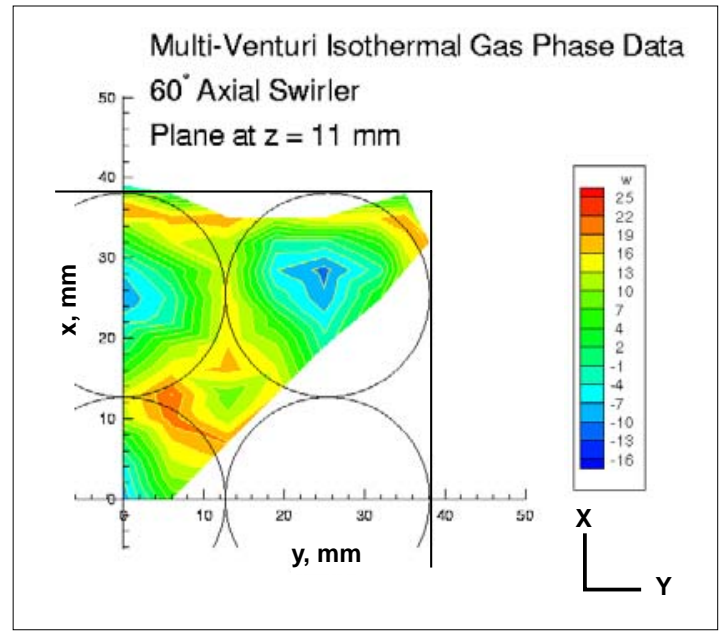

(c) Measurements

Figure 5.1.4. Results for $60^{\circ}$ Swirler at $\mathrm{Z}=11 \mathrm{~mm}$ Plane 


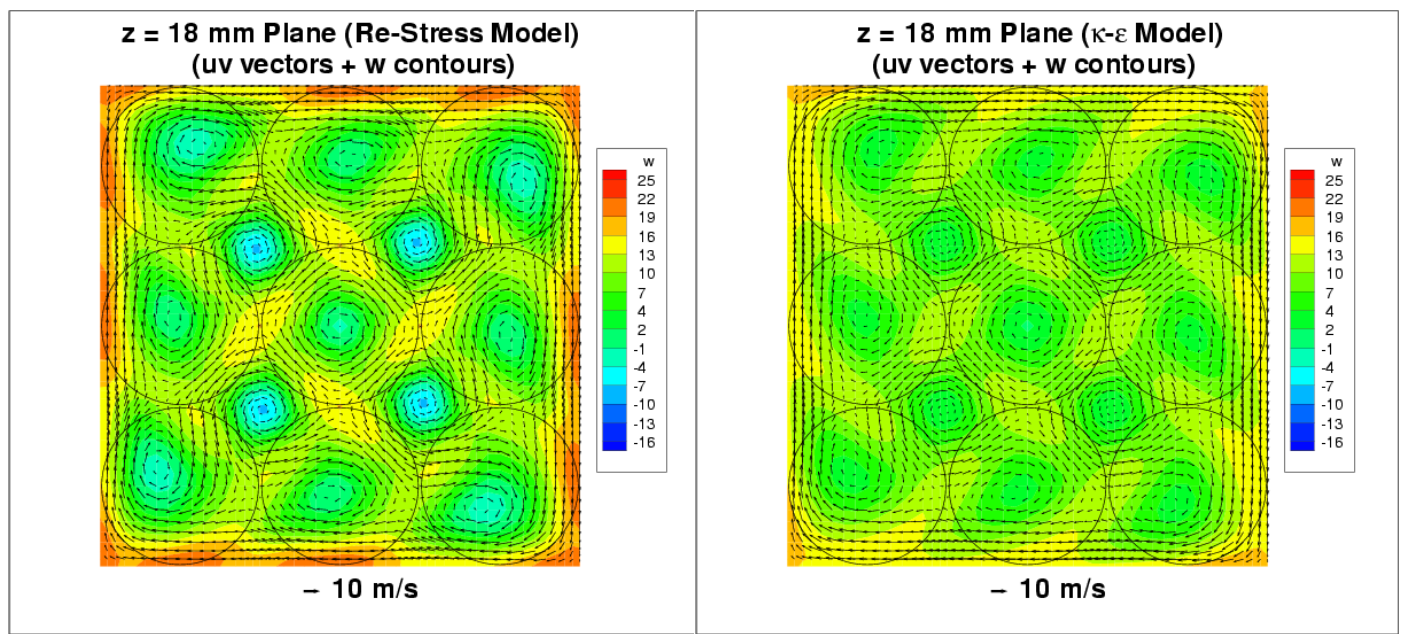

(a) RSTM

(b) $k-\varepsilon$

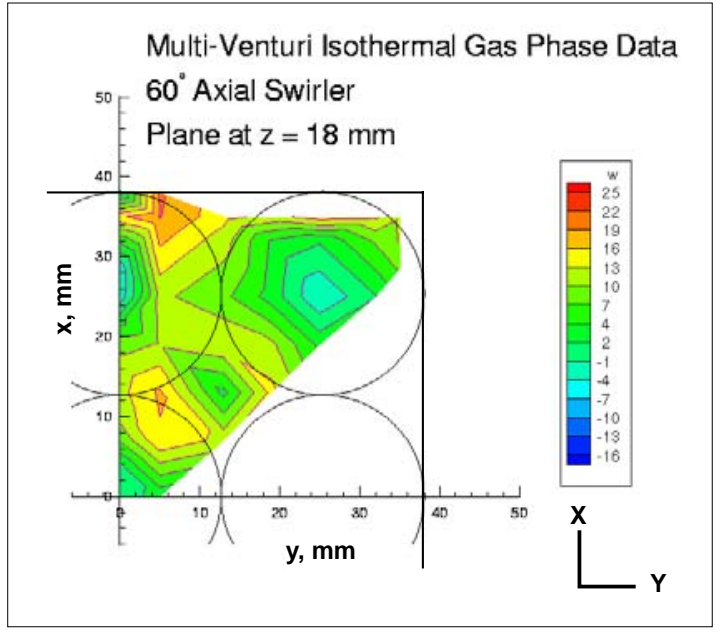

(c) Measurements

Figure 5.1.5. Results for $60^{\circ}$ Swirler at $\mathrm{Z}=18 \mathrm{~mm}$ Plane 


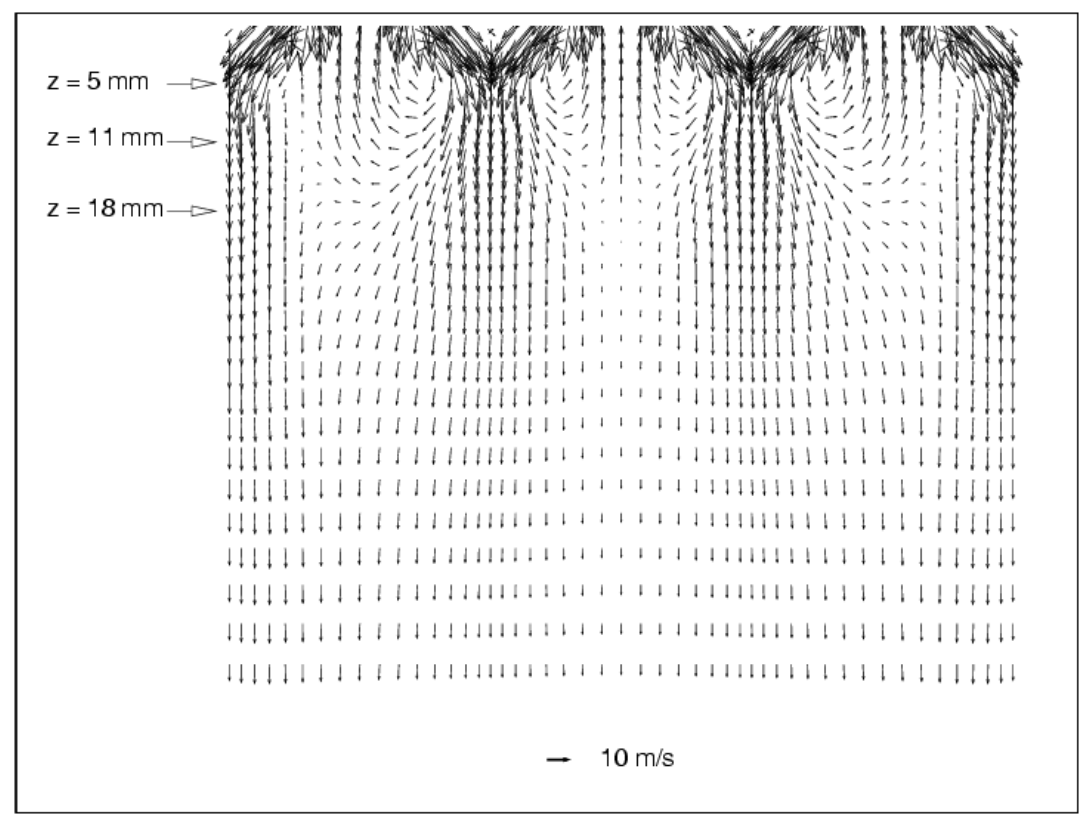

(a) RSTM

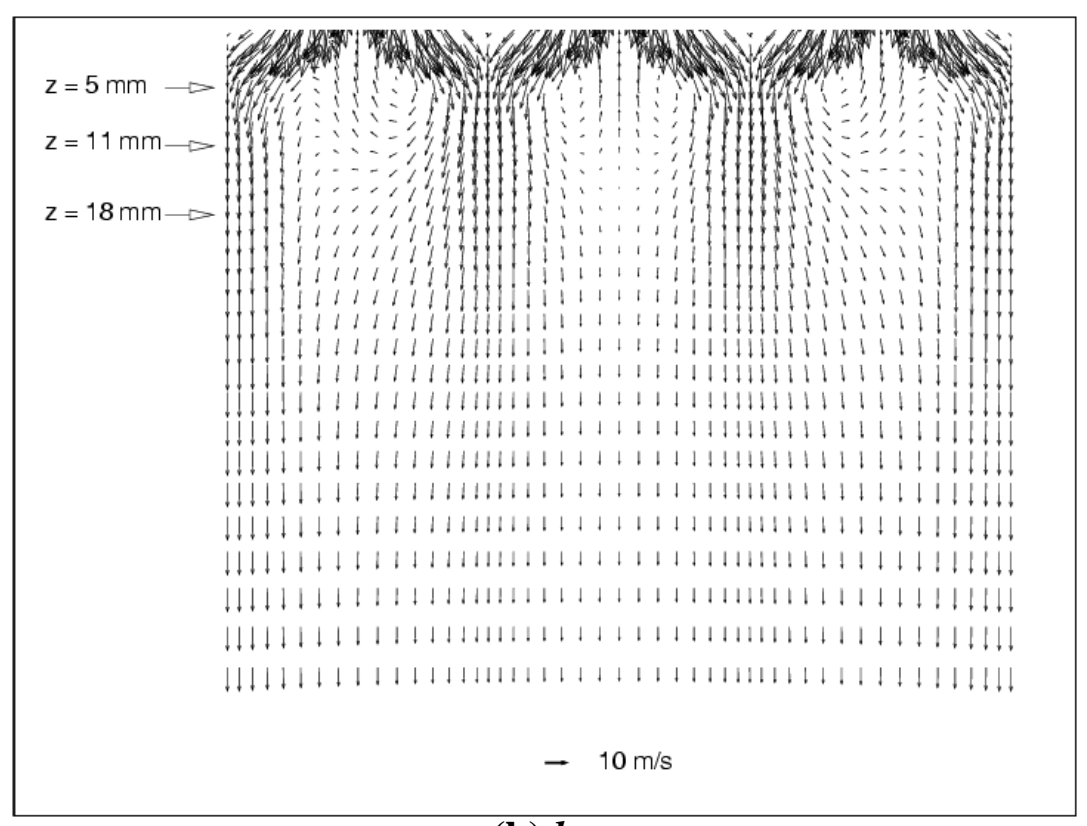

(b) $k-\varepsilon$

Figure 5.1.6. Velocity on $\mathrm{y}_{0}-\mathrm{y}_{0}$ Plane for $60^{\circ}$ Swirler 


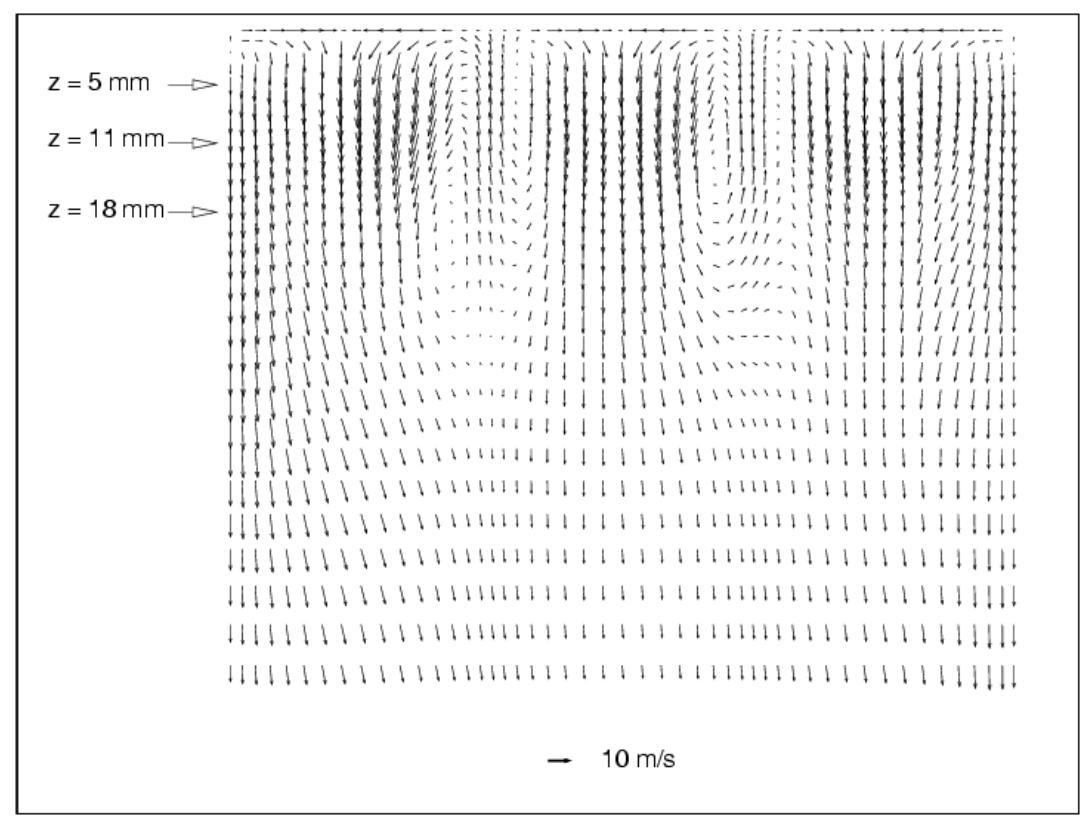

(a) RSTM

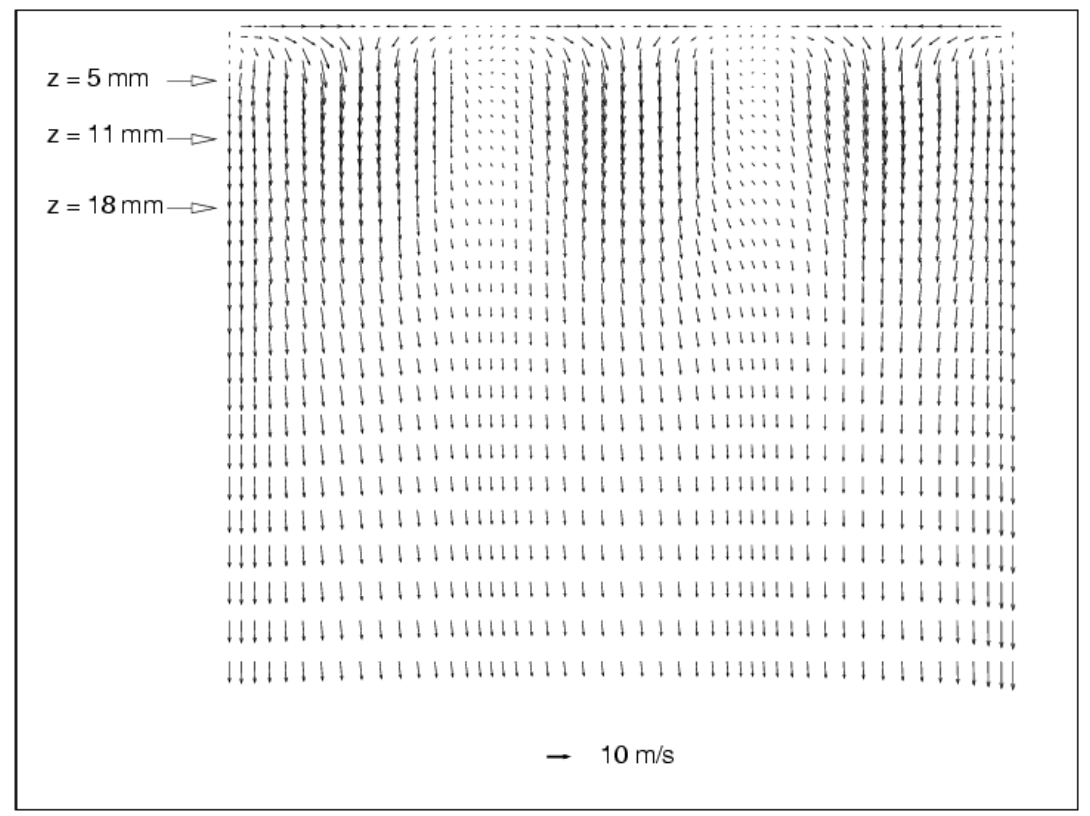

(b) $k-\varepsilon$

Figure 5.1.7. Velocity on $\mathrm{y}_{1}-\mathrm{y}_{1}$ Plane for $60^{\circ}$ Swirler 


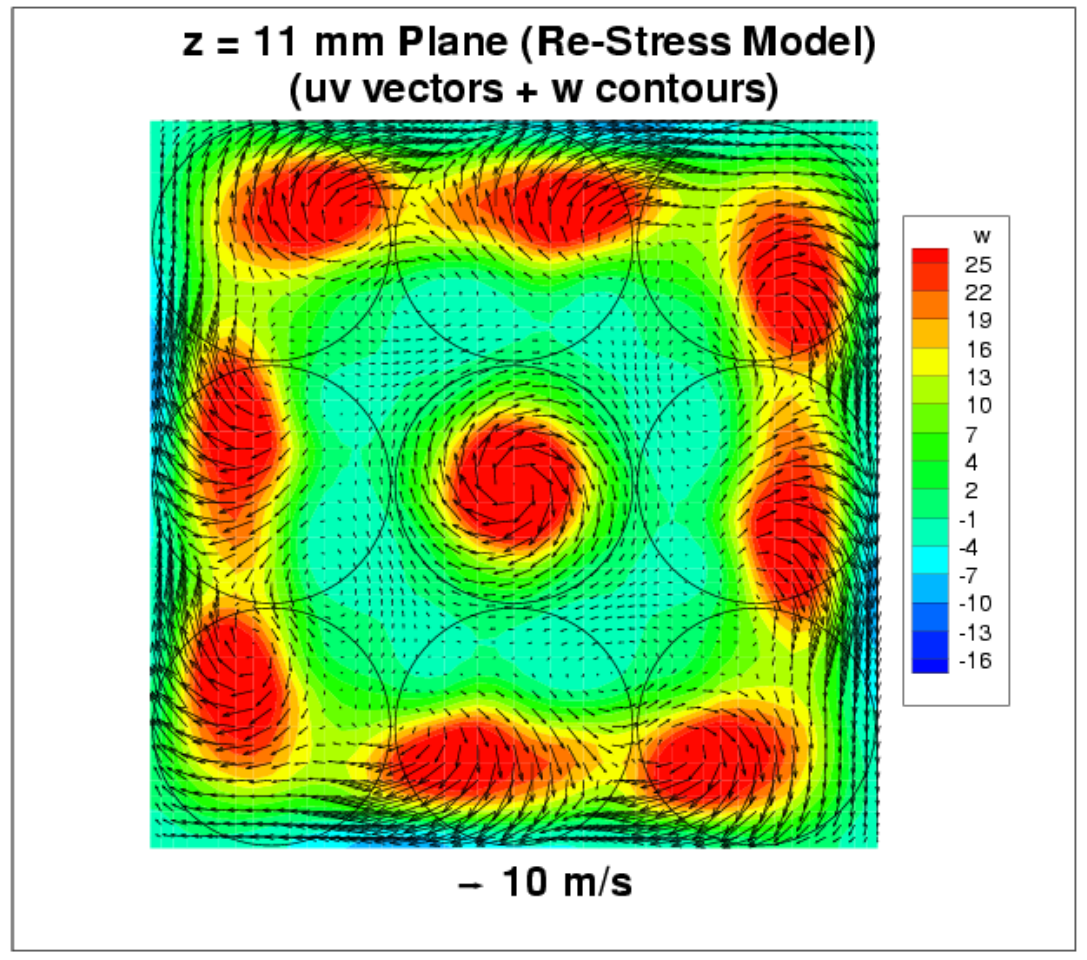

(a) RSTM

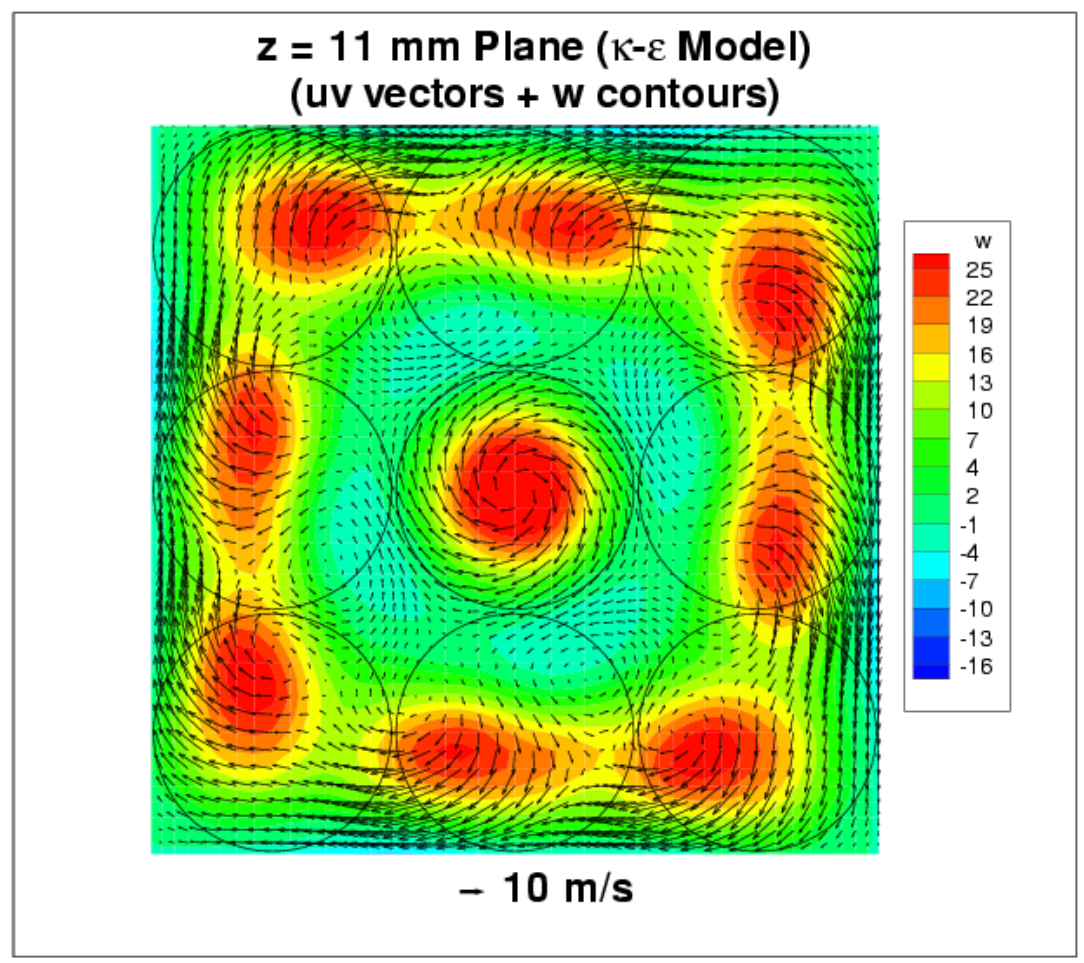

(b) $k-\varepsilon$

Figure 5.1.8. Results for $45^{\circ}$ Swirler at $\mathrm{Z}=11 \mathrm{~mm}$ Plane 


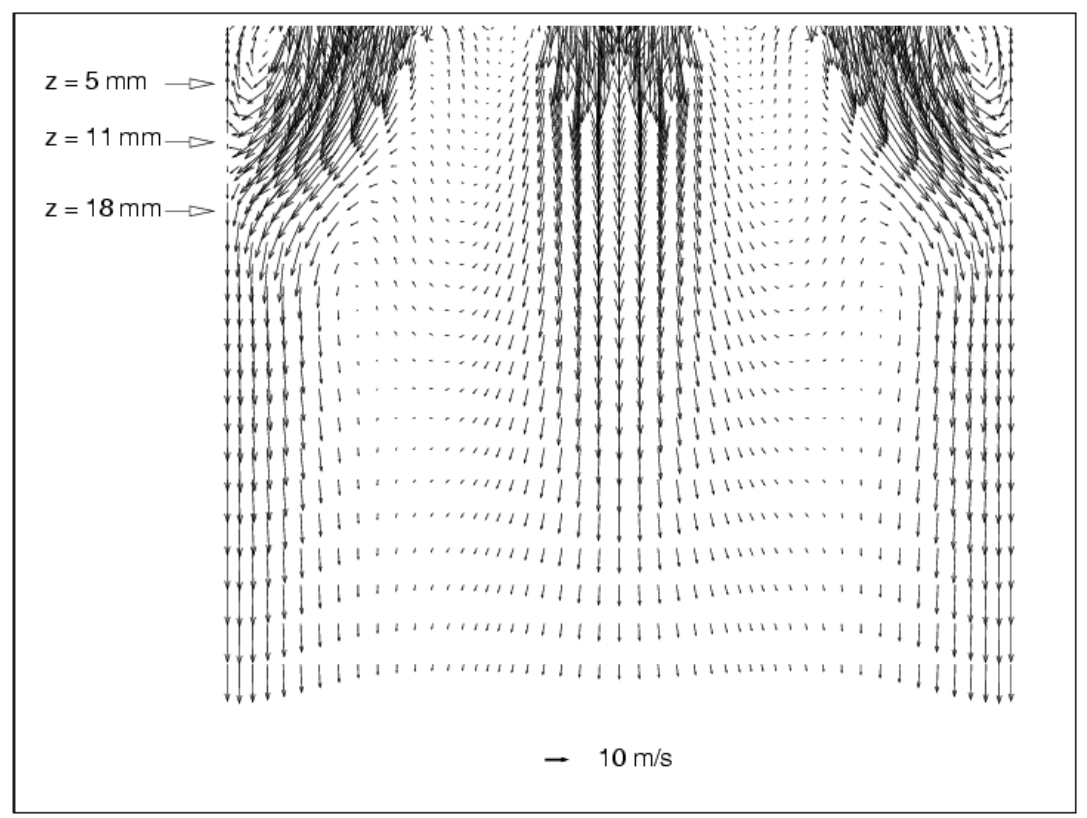

(a) RSTM

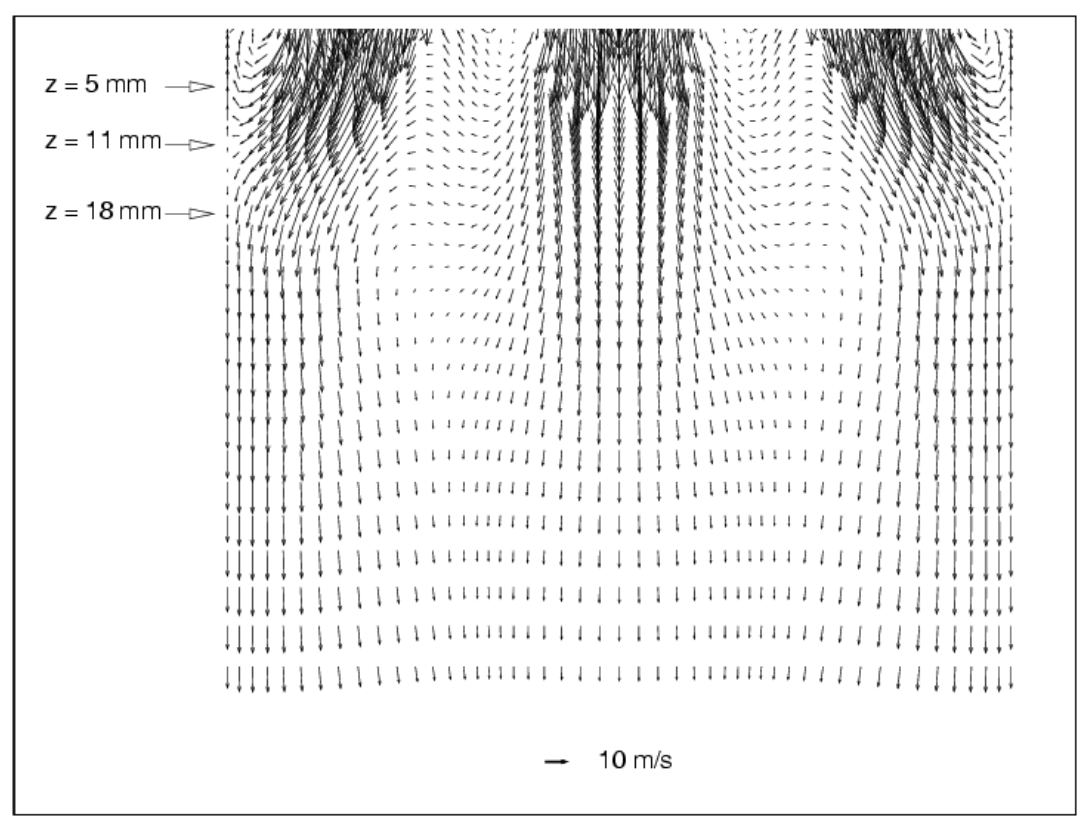

(b) $k-\varepsilon$

Figure 5.1.9. Velocity on $\mathrm{y}_{0}-\mathrm{y}_{0}$ Plane for $45^{\circ}$ Swirler 


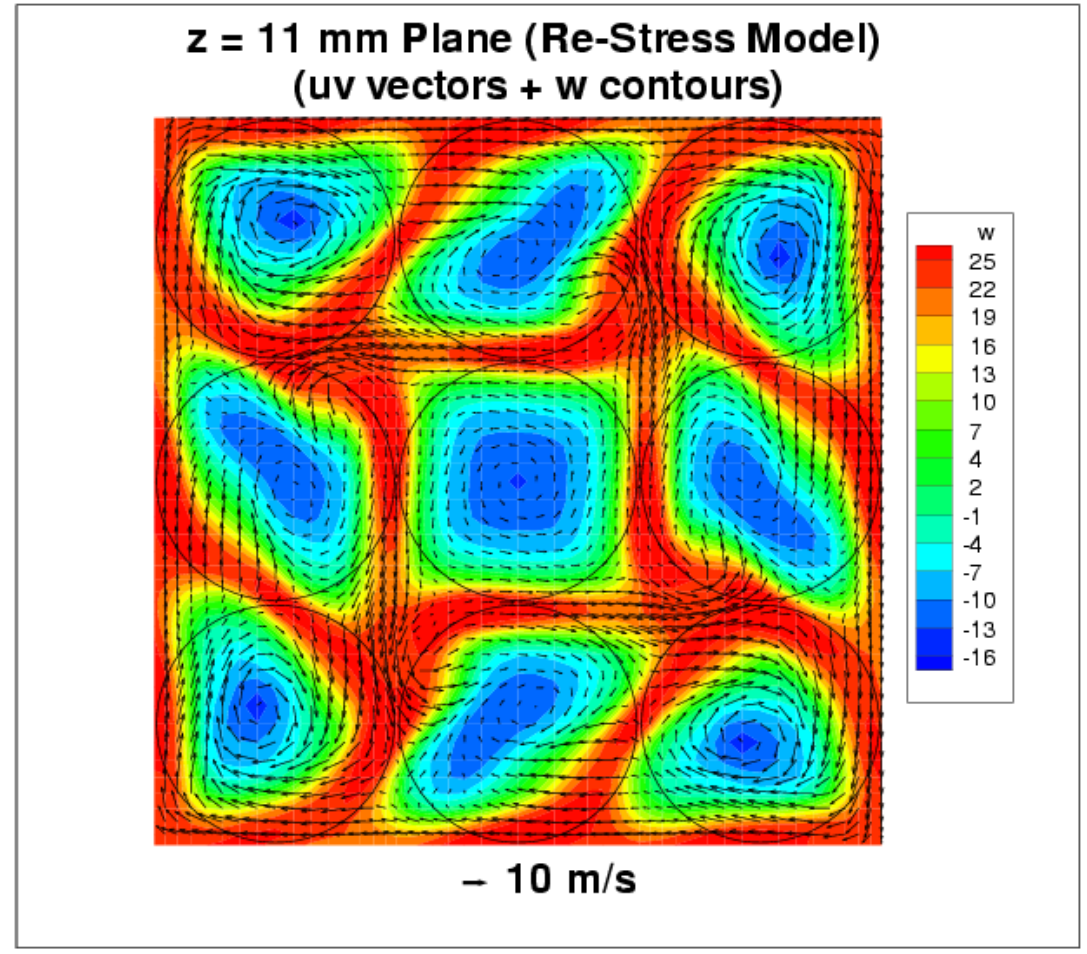

(a) RSTM

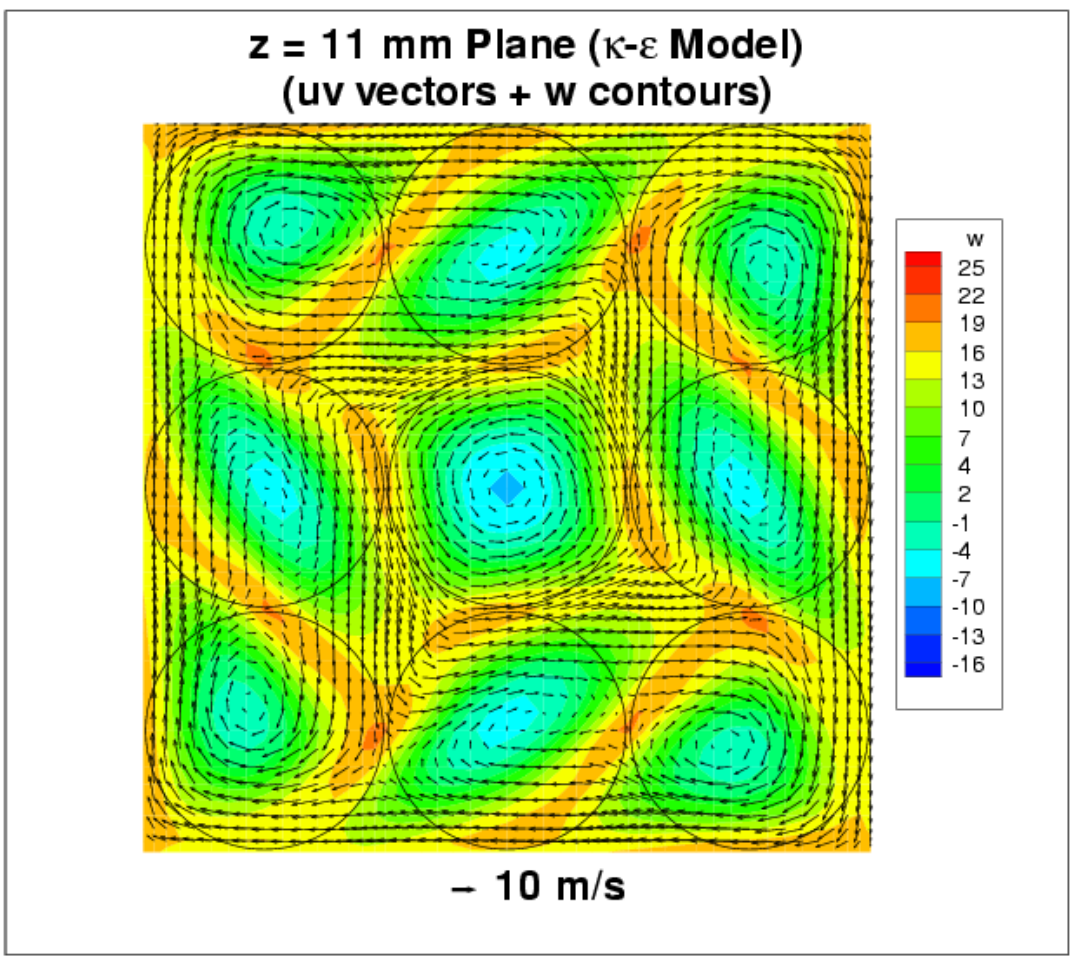

(b) $k-\varepsilon$

Figure 5.1.10. Results for $60^{\circ}$ Clockwise+Counterclockwise Swirler at $Z=11 \mathrm{~mm}$ Plane 


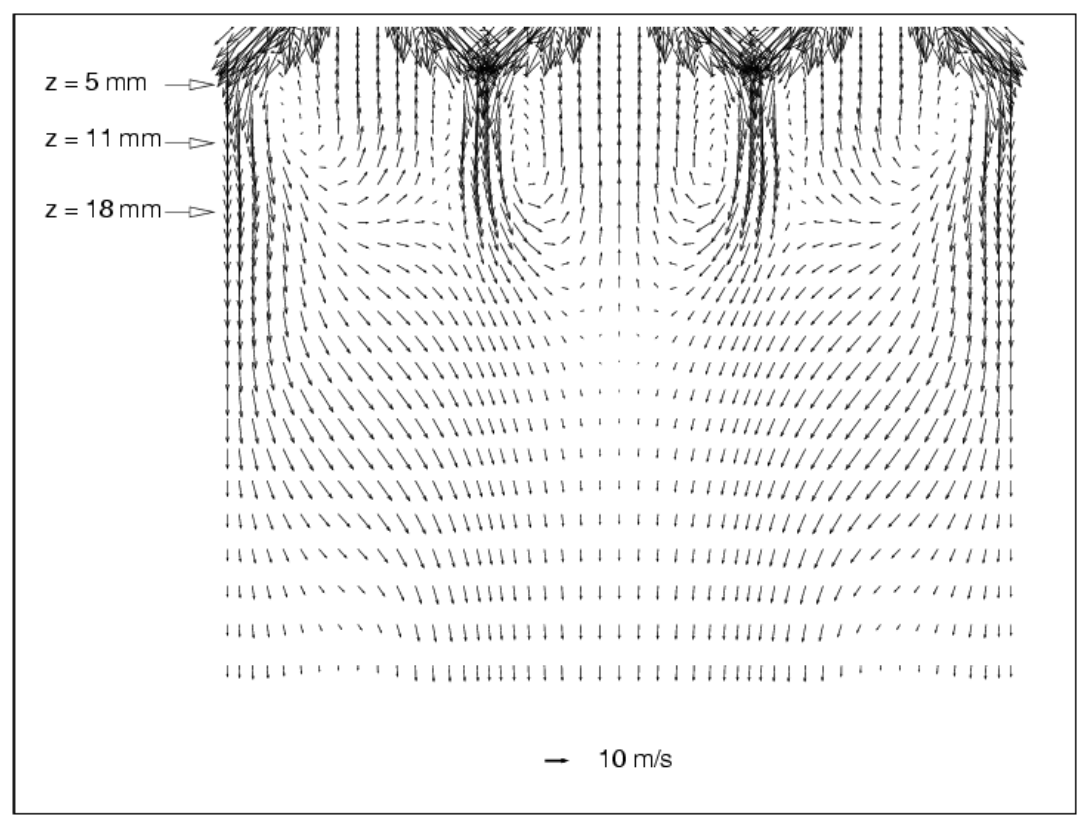

(a) RSTM

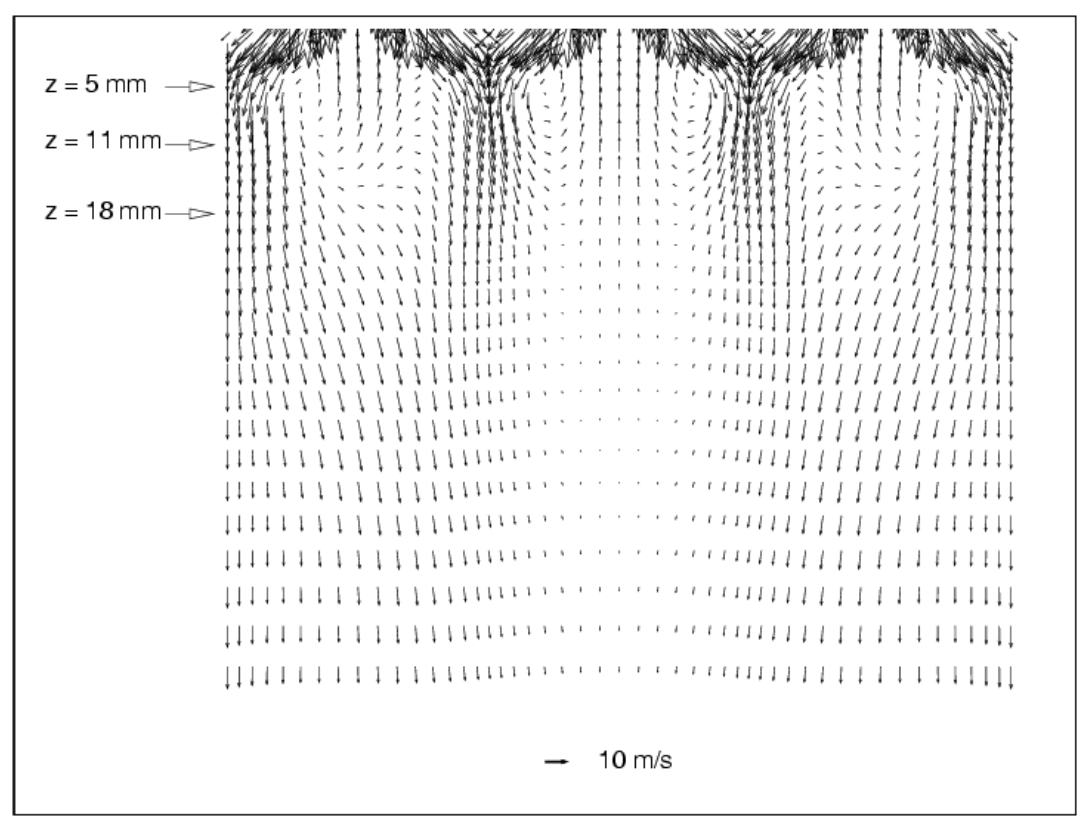

(b) $k-\varepsilon$

Figure 5.1.11. Velocity on $\mathrm{y}_{0}-\mathrm{y}_{0}$ Plane for $60^{\circ} \mathrm{CW}+\mathrm{CCW}$ Swirler 
Table 5.1: Inlet Turbulence Values

\begin{tabular}{ccccccccc}
\hline \hline $\bar{u}_{i n}$ & $k_{i n}$ & $\varepsilon_{i n}$ & $\tilde{u_{1} u_{1}}$ & $\tilde{u_{2} u_{2}}$ & $\tilde{u_{3} u_{3}}$ & $\tilde{u_{1} u_{2}}$ & $\tilde{u_{1} u_{3}}$ & $\tilde{u_{2} u_{3}}$ \\
\hline $0.05 V_{i n}$ & $1.5 \bar{u}_{i n}^{2}$ & $0.25 d$ & $0.25 k_{i n}$ & $0.65 k_{i n}$ & $1.1 k_{i n}$ & 0.0 & 0.0 & 0.0 \\
\hline \hline
\end{tabular}




\subsection{Lean-Premixed-Prevaporized (LPP) Combustor}

An experimental case of lean, pre-mixed, pre-vaporized combustion of Jet-A fuel in a duct is analyzed using a 16-species, 23-step kinetic mechanism in a time-march computational-fluid-dynamics code. The mechanism is primarily intended for modeling nitric oxide (NO) production. Computed concentrations of nitric oxide, carbon monoxide (CO) and carbon dioxide $\left(\mathrm{CO}_{2}\right)$ are compared to the experimental gas-sample measurements taken at discrete locations along the duct axis. Calculated results from a two-dimensional planar numerical simulation are compared with the experimental data for two configurations of the flame holder: an 8-hole and an 18-hole plate, both with $20 \%$ open area. Results are compared for three equivalence ratios, $f=0.45,0.60$ and 0.74 . The computed results for concentrations of $\mathrm{CO}_{2}$ and $\mathrm{NO}$ are consistent with the experimental data over the range of equivalence ratios considered, indicating that the mechanism is useful for modeling NO production in Jet-A combustion. As information on experimental uncertainty is not available for the experimental data, comparisons and assessments are somewhat qualitative.

\subsubsection{Introduction}

Modeling combustion in gas-turbine engines is a formidable task that requires detailed description of the turbulence, the kinetics of the chemical reactions, and the fuel spray if liquid injection is involved. The three are highly interdependent. The nature of the turbulence on both a micro- and macro-scale largely determines how effectively the fuel and air, mix and burn. The turbulence and chemical reactions, in particular, are usually strongly coupled. Modeling this complicated interaction of fluid dynamics and chemical 
kinetics probably requires a stochastic approach. For example, methods utilizing the probability-distribution function (PDF) to describe intermittent species concentrations and fuel-droplet distribution are currently being developed. Another complementary approach is to model, or resolve the flow field, to the scale of the large eddies, a method referred to as large-eddy simulation (LES). At this juncture, these methods have not yet reached the point of routine application, and engineers, therefore, must still resort to standard continuum-flow modeling for practical analysis. The usual approach is to apply a combustion model, be it a single-step reaction or a reaction of several steps, in conjunction with some turbulence model, typically a $k-\varepsilon$ model, to the Reynolds-averaged NavierStokes (RANS) equations. Many variations on this aproach can be found in the literature. Though this method does not capture the effects of the turbulence-combustion interactions on the fine, Kolmogorov scale, it, nonetheless, can yield some overall, practical engineering information.

Along these lines, this study focuses on the application of a reduced, or simplified, kinetic mechanism, designed primarily for concentrations of nitric oxide in Jet-A combustion, that was synthesized from a larger, detailed mechanism for propane (a detailed mechanism does not exist for Jet-A combustion). The mechanism is applied in a computational-fluid-dynamics (CFD) code, to model a pre-mixed, pre-vaporized experimental configuration where the turbulence is expected to be of a fairly fine scale and somewhat uniform, and no liquid spray is involved. The purpose of this exercise is to concentrate on the chemical kinetics as the primary combustion process, by specifically considering an experimental case that minimizes large-scale turbulent mixing and flame propagation, and intermittent mixing and burning. It is presumed that these experimental 
conditions approach that of a well-stirred reactor and therefore, reasonably amenable to a continuum analysis. The object here is to assess the effectiveness of the reduced mechanism in computing concentrations of the major pollutant species, and its usefulness in application to practical CFD in combustion analysis.

In prior studies [Kundu et al., 1998; Penko et al., 1999], two kinetic mechanisms are postulated that are designed to model hydrocarbon combustion and formation of the major pollutant species: nitric oxide, $\mathrm{NO}$, and carbon monoxide, $\mathrm{CO}$, with emphasis on NO. In this study, the larger mechanism consisting of 16-species and 23-steps, with some minor modifications, is used to demonstrate the effectiveness of modeling combustion of Jet-A fuel in a pre-mixed, pre-vaporized experimental configuration. The mechanism is primarily designed to compute the production of $\mathrm{NO}$, and is applied in a code that solves the RANS equations, with a Reynolds-stress turbulence model. Computed results for NO, $\mathrm{CO}$ and $\mathrm{CO}_{2}$ are compared to experimental measurements of the same species taken with a gas-sample probe at locations along the axis of the duct.

\subsubsection{The CFD Code}

The computer code used in this study is a modified version of KIVA-3 [Amsden, 1993] that is generally intended for computing two- and three-dimensional compressible flows, with combustion reactions, for gaseous or liquid-fuel sprays, in piston-engine chambers. It is also well suited for through-flow combustors of the type used in gasturbine engines. Detail of the code and the implementation of a Reynolds-stress turbulence model can be found in Chapter 4 of this dissertation. 


\subsubsection{The Kinetic Mechanism}

Jet-A fuel is actually a mixture of various hydrocarbons that includes, mostly, paraffins and olefins [Coordinating Research Council, 1983]. For example, a GCMS (gas chromatograph-mass spectrometer) analysis of Jet-A gives a mixture that is approximately $49 \%$ dimethylnaphthalene, $36 \%$ hexadecane and $15 \%$ hexadecene. Given the complexity of this mixture, there is no kinetic model available that can describe the complete combustion process and, therefore, no detailed mechanism exists that can be used as a starting point for developing a reduced mechanism. Rather, the basic assumptions in developing the Jet-A mechanism are:

1) Jet-A has the formula $\mathrm{C}_{12} \mathrm{H}_{23}$ based on a simple carbon-hydrogen ratio of the constituent hydrocarbons, and

2) The reactions are the same as for propane once the fuel breaks down into simpler fragments in an initial, global reaction, given by:

$$
\mathrm{C}_{12} \mathrm{H}_{23} \rightarrow 12 \mathrm{CH}+11 \mathrm{H}
$$

This global reaction for the initial fuel fragmentation is the first in a series of global and bi-molecular reactions (the complete mechanism is given in Appendix B), formulated along the lines originally proposed by Edelman and Fortune [1969]. The mechanism includes the formation of nitric oxide by the well-known Zeldovich reactions for thermal NO, reactions for prompt NO by Fenimore [1971] for both lean and rich combustion, and formation of $\mathrm{NO}$ by the $\mathrm{N}_{2} \mathrm{O}$ route. The mechanism presented here is derived from a detailed mechanism using the sensitivity method contained in Bittker's code for chemical kinetics [Bittker, 1996]. The starting, detailed mechanism is first created by adding a global reaction for propane to the extensive methane mechanism of Miller and Bowman 
[1989]. In derivation of the reduced mechanism, several approximations are made to limit the number of reacting species:

1) Only two hydrocarbon fragments, $\mathrm{CH}$ and $\mathrm{C}_{2} \mathrm{H}_{2}$, are included,

2) A single, global reaction simulates a multitude of hydrocarbon-fragmentation reactions for the fuel pyrolysis,

3) Formation of nitrogen oxides through the $\mathrm{N}_{2} \mathrm{O}$ route is simulated by a single, global reaction (reaction 3 of Appendix B).

Although a reduced mechanism cannot possibly describe the numerous and complex pyrolytic reactions for a liquid-petroleum fuel, which consists of many hydrocarbons, an attempt is, nonetheless, made to include some effects of the more important pyrolitic steps in the initial, fuel-breakdown reaction. Lumping the complex pyrolytic steps gives efficiency to the combustion calculations, and makes the burden on computer memory much less than for schemes with kinetic models that include heavier hydrocarbon fragments [Krollrack and Aceto, 1973].

\subsubsection{Experimental Configuration}

The experimental configuration is a duct used for experimental measurements in combustion of pre-mixed, pre-vaporized Jet-A fuel at a pressure of $6.5 \mathrm{~atm}$ [Semerjian and Vranos, 1976]. The configuration is modeled with the KIVA-3 computer code that includes the Jet-A mechanism described in the prior section. The configuration is illustrated in the schematic of Figure 5.2.1. The basic geometry and flow parameters are listed in the following table: 
Table 5.2: Geometry and Flow Parameters

\begin{tabular}{|l|r|}
\hline Height & $3.8 \mathrm{~cm}$ \\
\hline Width & $7.6 \mathrm{~cm}$ \\
\hline Overall Length & $32 \mathrm{~cm}$ \\
\hline To Flame Holder & $5 \mathrm{~cm}$ \\
\hline Flame Holder Thickness & $0.64 \mathrm{~cm}$ \\
\hline Flame Holder 1 & 8-hole, 2 rows of 4 \\
\hline Flame Holder 2 & 18-hole, 3 rows of 6 \\
\hline Flame Holder Open Area & $20 \%$ \\
\hline Inlet Flow Velocity & $20 \mathrm{~m} / \mathrm{s}$ \\
\hline Inlet Pressure & $6.5 \mathrm{~atm}$ \\
\hline Inlet Temperature & $750 \mathrm{~K}$ \\
\hline Equivalence Ratios & $0.45,0.60,0.74$ \\
\hline
\end{tabular}

The experimental data consists primarily of concentrations (ppm by mole fraction) of $\mathrm{NO}, \mathrm{CO}$ and $\mathrm{CO}_{2}$, and gas temperatures in the combustion products, taken with a gassample probe at discrete, axial locations along the flame tube. The residence time for this configuration is about $3 \mathrm{~ms}$ in the volume starting immediately downstream of the flame holder and extending to the last measurement station located $25 \mathrm{~cm}$ from the flame holder. This residence time corresponds to an average gas velocity of about $60 \mathrm{~m} / \mathrm{s}$.

For the computational simulation, two-dimensional grids for both flame-holder plates are used. The grid for the 8-hole plate is illustrated in Figure 5.2.2, and for the 18hole plate in Figure 5.2.3 (Grids for 3-D simulations of these configurations were also created but the numerical solutions were not complete at the time of this writing). For the 
2-D simulations, the multi-holed flame holders are modeled as plates with slots of open area equal to the holed plates. In the CFD simulations, a uniform velocity profile and uniform levels of turbulence kinetic energy are imposed at the inlet, and adiabatic walls are assumed.

\subsubsection{Results}

Some basic results from the CFD analysis are given in the following table.

Table 5.3: 18-Hole Plate, $25 \mathrm{~cm}$ Measurement Station

\begin{tabular}{|c|r|r|r|r|r|r|}
\cline { 3 - 7 } \multicolumn{2}{c|}{} & \multicolumn{2}{c|}{ NO (ppm) } & \multicolumn{2}{c|}{ CO $\left(\times 10^{-3}\right)$} \\
\hline$\phi$ & dp (\%) & T $(\mathrm{K})$ & Calc. & Expt. & Calc. & Expt. \\
\hline \hline 0.74 & 4.1 & 2232 & 218 & 184 & 1.7 & 1.0 \\
\hline 0.60 & 3.9 & 2034 & 53 & 38 & 0.2 & 0.5 \\
\hline 0.45 & 3.8 & 1780 & 5.5 & 5.0 & - & 0.1 \\
\hline
\end{tabular}

Although static pressure drop ( $\mathrm{dP} \%)$ was not specifically listed in the experimental paper, the computed results are well within reason for this configuration and are somewhat typical of combustion testing. Also listed in the table are the experimental values for concentrations of $\mathrm{NO}$ and $\mathrm{CO}$ at the last measurement station in the duct, along with the corresponding calculated values at the same location. The concentrations of $\mathrm{NO}$ and $\mathrm{CO}$ are in mole fraction where the $\mathrm{CO}$ is given in parts per thousand (10-3).

Comparisons of calculated results with the experimentally measured concentrations, at all axial stations, for three equivalence ratios, are given in Figures 5.2.45.2.6 for the 18-hole flame-holder that is simulated in the CFD analysis by the 3-slot, 2-D 
mesh. In these figures, the computed results are the solid lines and the experimental data, the symbols. Also in these figures, the axial distance is referenced to the downstream end of the flame holder. Figure 5.2.7 shows temperatures for the 3-slot flame-holder for $\mathrm{f}=$ 0.60 and 0.45 . Comparisons of $\mathrm{NO}, \mathrm{CO}$ and $\mathrm{CO}_{2}$ concentrations for the 8-hole flameholder, simulated by the 2-slot mesh, for $\mathrm{f}=0.60$, are given in Figure 5.2.8 and the temperature in Figure 5.2.9. The experimental data is presented without error bars as no information was available on experimental uncertainty.

\subsubsection{Discussion of Results}

In general, the computed results for concentrations of $\mathrm{CO}_{2}$ compare quite well with the experimental measurements for all equivalence ratios. The close agreement for $\mathrm{CO}_{2}$ is to be expected, as this is rather basic to any combustion calculation, regardless of the complexity of the mechanism. The NO calculated results also track the experimental measurements reasonably well at all equivalence ratios. At the last measurement station, the percent difference with respect to the measured values are $10 \%$ for $\mathrm{f}=0.45,18 \%$ for $\mathrm{f}=0.74$, and $33 \%$ for $\mathrm{f}=0.60$. This is considered reasonable given the complexity of modeling NO production in combustion processes.

The calculated concentrations of $\mathrm{CO}$ do not track the experimental results as well as the $\mathrm{CO}_{2}$ or NO. Fairly large discrepancies occur at all equivalence ratios. This is not unexpected as the mechanism is not necessarily designed and tuned for $\mathrm{CO}$ production. Furthermore, some of the $\mathrm{CO}$ measurements could be questionable. For one, the measurement system could be at the low-end of its usable range at the lean conditions, especially for $\mathrm{f}=0.45$ where measurement error could be substantial, and two, other 
carbon-containing species may be included in the measurement of $\mathrm{CO}$ that are not present in the kinetics calculation. It is well known that $\mathrm{CO}$ measurements in lean combustion can have large bands of uncertainty, so these comparisons are somewhat tenuous at best and should not be taken at face value. Nonetheless, the $\mathrm{CO}$ production in the mechanism is being addressed with a further modification.

As the primary interest in combustion analysis for gas turbines is usually temperatures at liner surfaces and the turbine-inlet station, and overall emission levels, the general agreement between calculated and experimental data indicates that the combination of the CFD code and kinetics model is quite satisfactory for these purposes. Furthermore, the code and kinetic mechanism seems to do well in calculating concentrations of NO, which the mechanism was specifically intended. The differences between computed and experimental values, however, cannot be adequately addressed, quantitatively, without further information on experimental uncertainty and code accuracy. Therefore, the present discussion is reserved primarily to qualitative comparisons instead of quantitative ones.

It should be noted that the CFD modeling does not account for partial mixing of the burned and unburned gases in the vicinity of the flame, beside what occurs from the fluid dynamics and turbulence model. Nor does it account for any non-uniformity in the mixture of fuel and air upstream of the flame holder. Furthermore, there are no measurements of these parameters in this particular experimental study. Rather, it is assumed that the fuel is fully vaporized, thoroughly mixed with the air, and that the fuelair mixture is completely uniform before reaching the flame holder, and that, because of the multi-holed plate, the burning is fairly uniform. Under these assumptions, the CFD 
model should be a reasonably valid description of the experimental case, and the comparison of experimental and computed results, a credible test of the Jet-A mechanism.

\subsubsection{Computer Execution}

Some general information on the Unix machine used to generate the calculated results is given in Table 5.4.

Table 5.4: Machine Description

\begin{tabular}{|l|l|}
\hline Type & SGI Power Onyx \\
\hline Memory & $4 \mathrm{~GB}$ \\
\hline CPUs & 12 \\
\hline Processors & R10000 @ $195 \mathrm{MHz}$ \\
\hline Main Memory & $4096 \mathrm{MB}$ \\
\hline Cache & $32 \mathrm{kB}$ \\
\hline
\end{tabular}

Although a residual is not usually monitored, temperature and NO concentration at a specified sample plane are checked to insure that the solution converges to a steady-state value. An example of this is given in Figure 5.2.10, where shown are the averaged temperature and NO concentrations in the z-plane, at a distance of $25 \mathrm{~cm}$ from the flame holder. In each numerical case, an initial ignition phase is applied, whereby energy is added to the flow in a specified volume downstream of the flame holder. The ignition is typically applied for a physical time of $5 \mathrm{~ms}$. A run of 25,000 iterations, as shown in Figure 5.2.10, takes about $10 \mathrm{hrs}$ of CPU time. For this case, the average CPU-time/cell/ iteration is approximately $704 \mathrm{~ms}$. 


\subsubsection{Conclusion}

The applicability of a reduced mechanism for calculating combustion of Jet-A fuel is demonstrated by modeling an LPP flame-tube. The simulation is done with a modified version of the KIVA-3 code that accepts the kinetic mechanism detailed in Appendix B. The computational results for the concentrations of nitric oxide, which the mechanism is specifically intended, compare reasonably well with the experimental measurements. From a computational standpoint, the CFD code in combination with the mechanism works quite satisfactorily, at least on the pre-mixed, pre-vaporized combustion problem presented in this study. Convergence of the problem is attained in acceptable CPU times on an SGI Power Onyx system.

As a final comment, it should be noted that this study does not represent a validation, verification or calibration of the code or the kinetics model, as the experimental data is not sufficiently defined with error uncertainty nor the code with numerical accuracy. Rather, it is primarily an indication that the kinetics model may be useful for engineering analysis of Jet-A combustion, given the reasonable agreement with the experimental data used for comparison. Further work needs to be done to actually verify and validate the mechanism as a description of Jet-A combustion. 


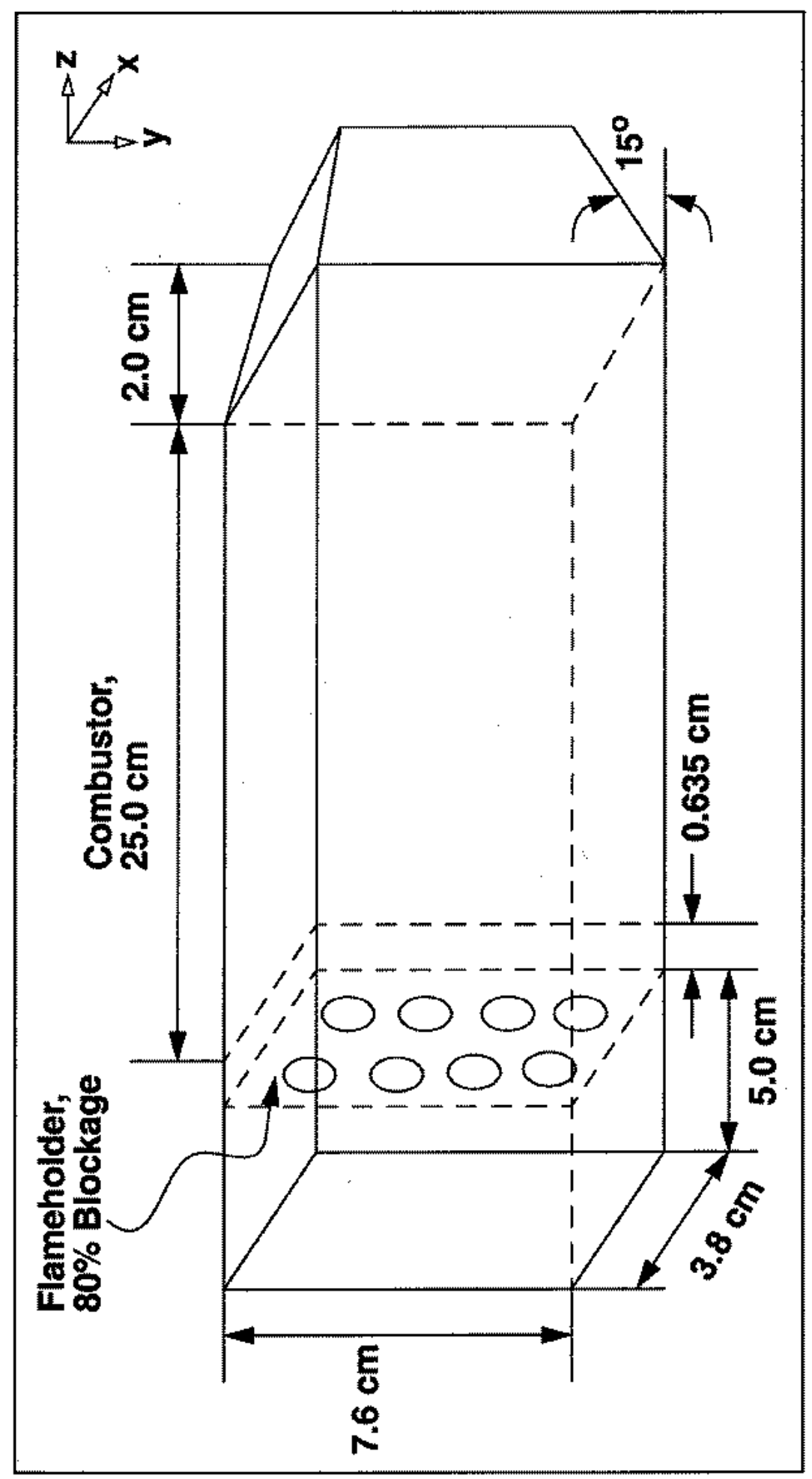

Figure 5.2.1. Schematic of the Test Duct, Combustor 

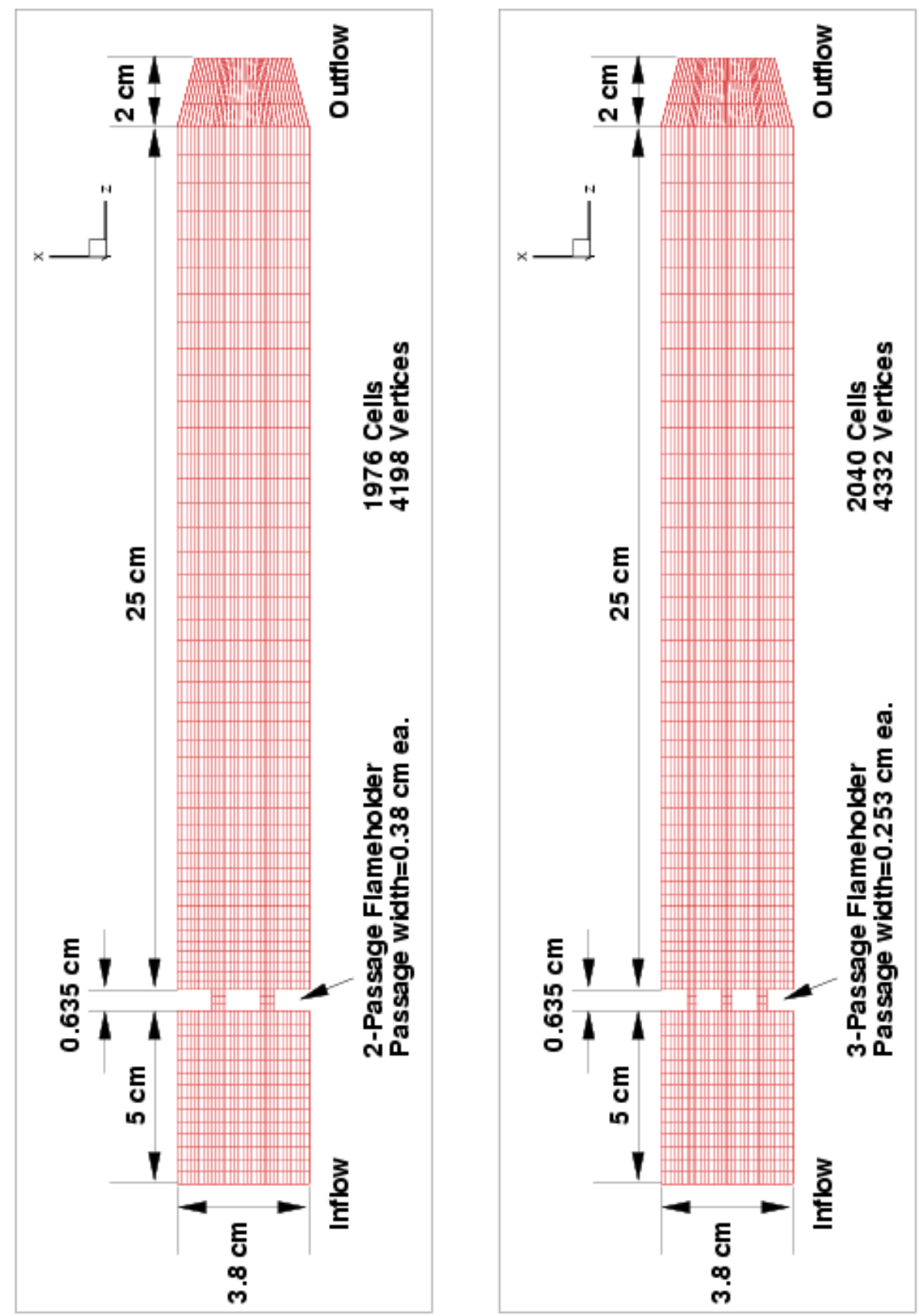

Figures 5.2.2 \& 5.2.3. Computational Mesh (2-D) for the Combustor with 8-Hole \& 18-Hole Flame-Holder 


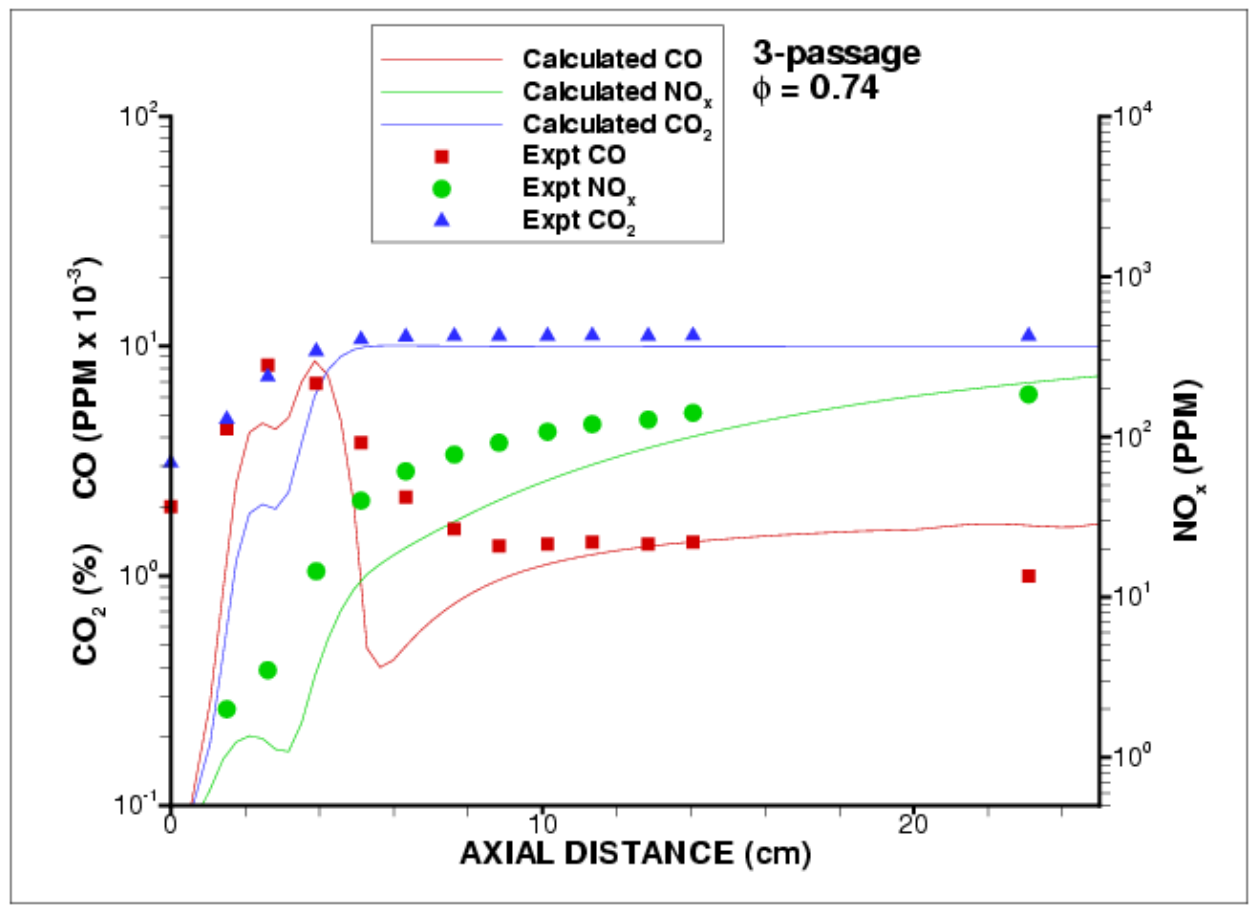

Figure 5.2.4. Results for 18-Hole Flame-Holder, $\phi=0.74$

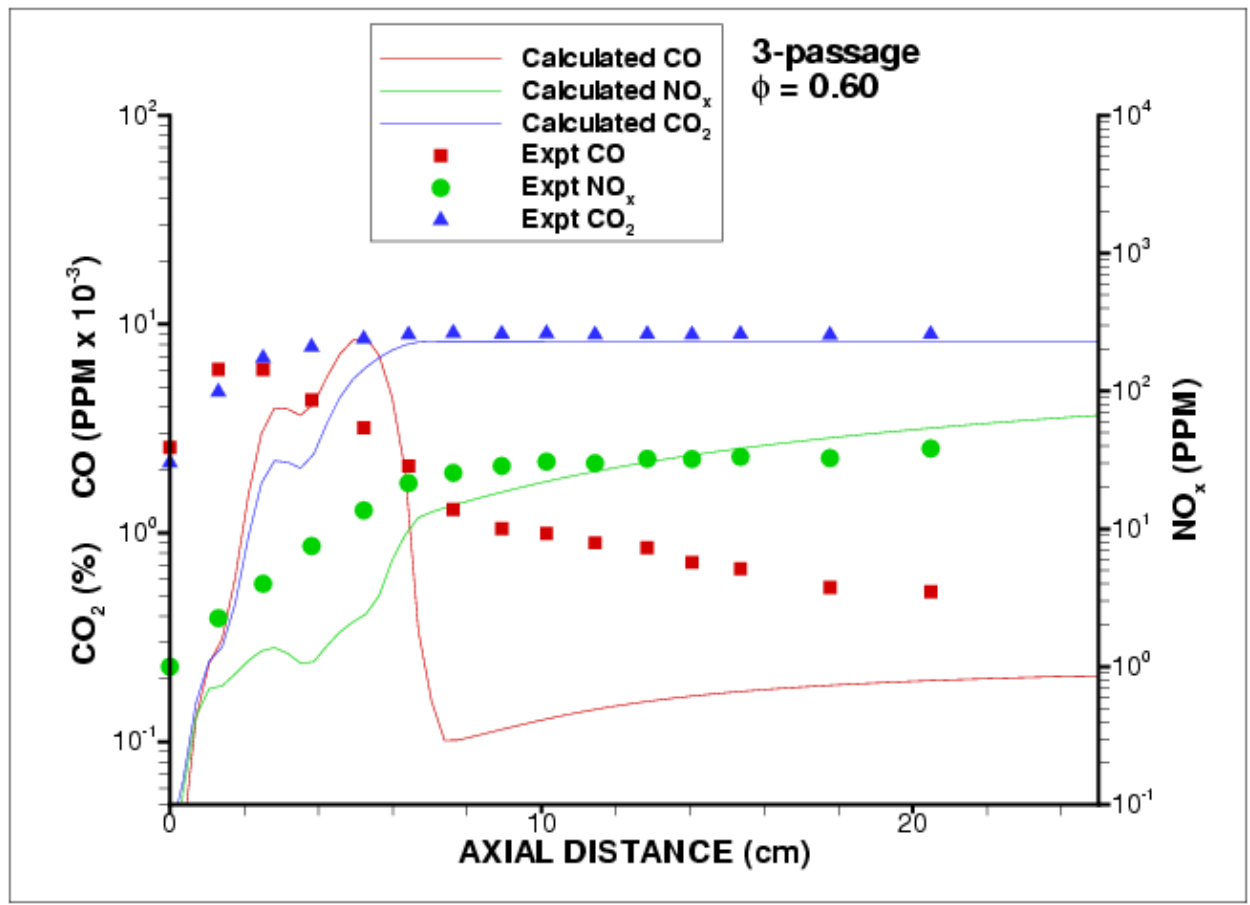

Figure 5. Results for 18-Hole Flame-Holder, $\phi=0.60$ 


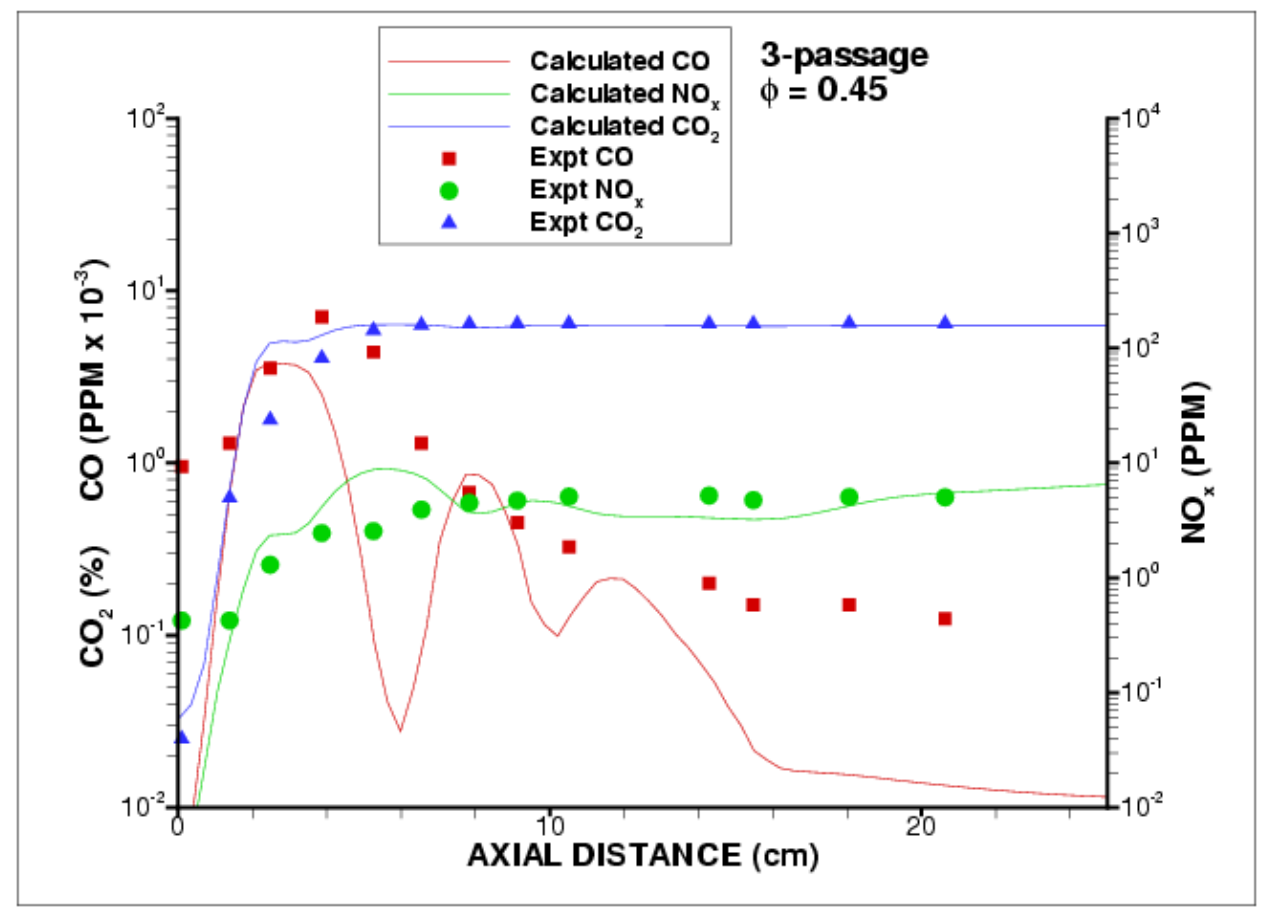

Figure 6. Results for 18-Hole Flame-Holder, $\phi=0.45$

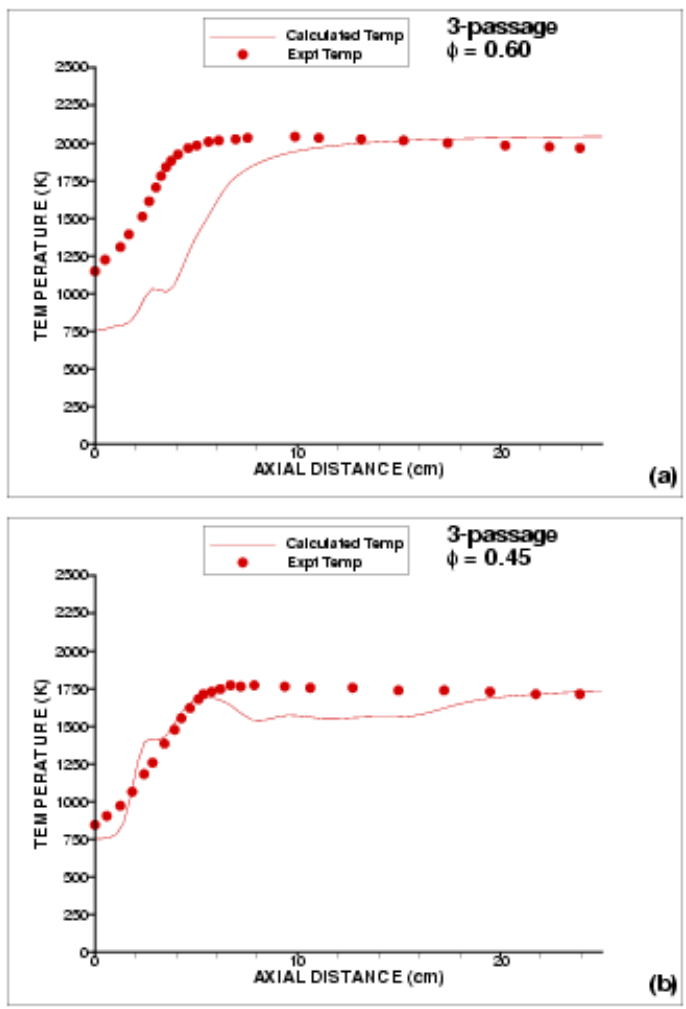

Figure 7. Temperature Profiles for 18-Hole Flame Holder: (a) $\phi=0.60$, (b) $\phi=0.45$ 


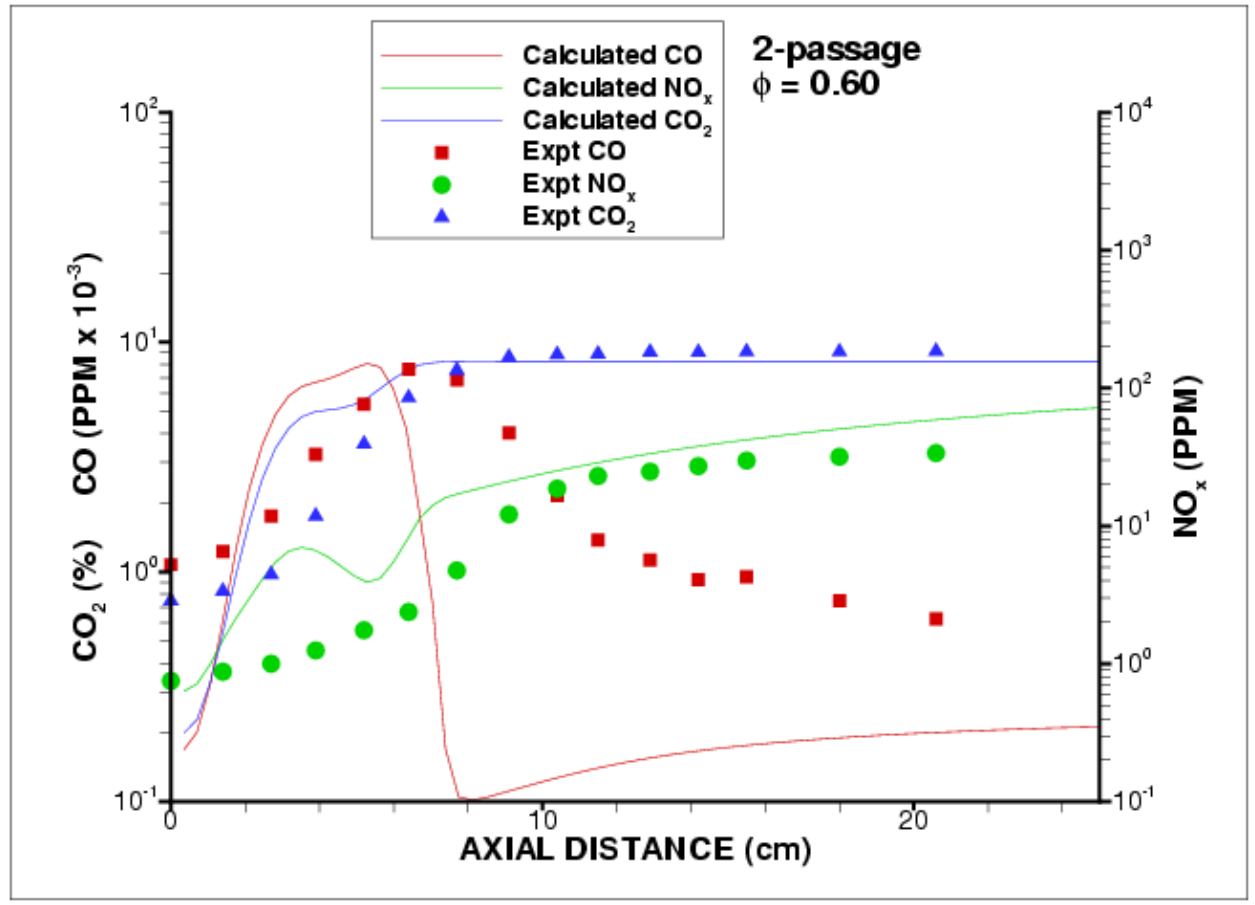

Figure 8. Results for 8-Hole Flame-Holder, $\phi=0.60$

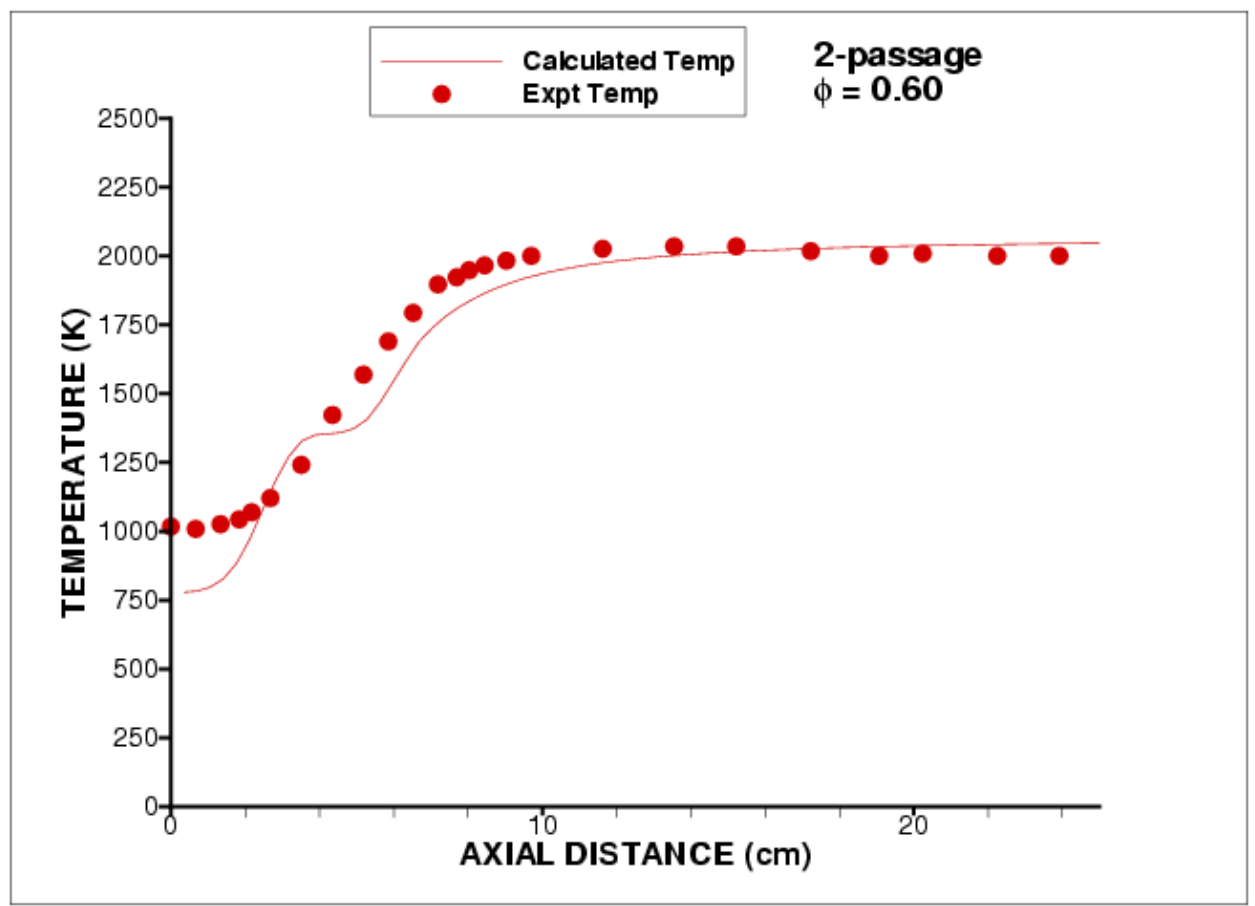

Figure 9. Temperature Profile for 8-Hole Flame Holder, $\phi=0.60$ 


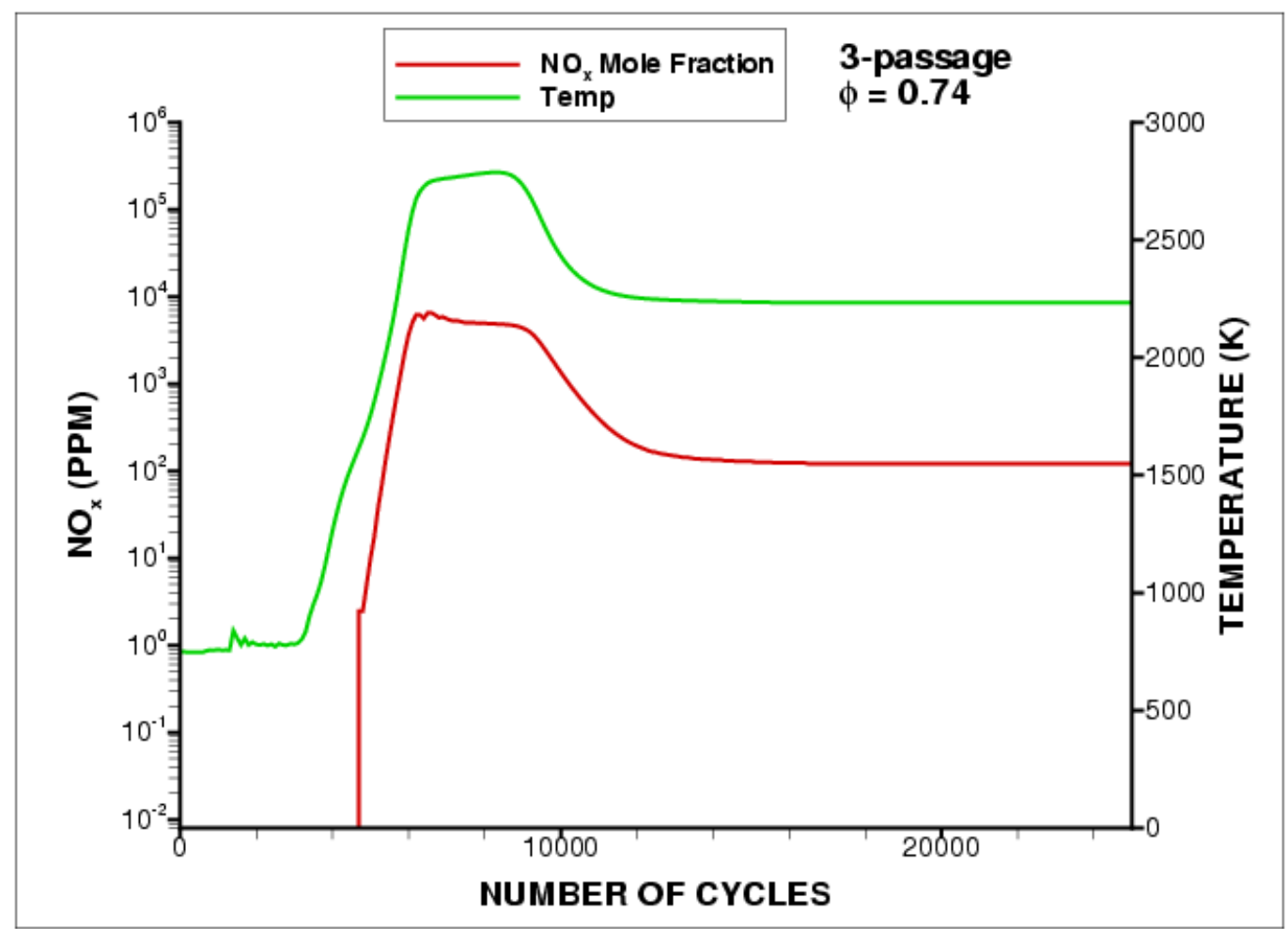

Figure 5.2.10. Temperature and NO Mole-Fraction Convergence History 


\subsection{LDI Combustor with Discrete-Jet Swirlers}

The flow field in a lean-direct injection (LDI) combustor with discrete-jet swirlers is described and analyzed using a computational-fluid-dynamics (CFD) code with a Reynolds-stress turbulence model (RSTM). The results from the RSTM are compared to time-averaged laser-Doppler velocimetry (LDV) data, as well as results from the National Combustion Code (NCC) that has a cubic non-linear $k$ - $\varepsilon$ turbulence model, and from the KIVA code using the standard $k-\varepsilon$ model. The comparisons of results indicate that the RSTM accurately describes the flow details and resolves recirculation zones and high velocity-gradients while the $k-\varepsilon$ models are unable to capture most flow structures. This confirms that, within the Reynolds averaging approach, the higher-order RSTM is preferred for simulating complex flow fields where separations, strong anisotropy, and high swirl are present.

\subsubsection{Introduction}

Flame stability over a wide range of operating conditions is a basic requirement for through-flow combustion devices, such as gas turbine combustors. One common practice used to stabilize combustion is generation of swirling flow that entrains and recirculates a portion of the hot combustion products. For direct-injection engines, recirculation zones also enhance the mixing of the incoming air with fuel. This effect is readily accomplished by the use of swirlers, such as discrete-jet swirlers, surrounding the fuel injector [Lefebvre, 1983].

Air swirlers are often used in many types of aircraft engines. In the lean direct injection (LDI) concept, a key feature is the injection of finely atomized fuel into the high- 
swirling airflow at the combustor dome that provides a homogenous, lean fuel-air mixture. This allows for a better combustion efficiency and the reduction of NOx and other pollutants. Another advantage of the LDI concept is that, with proper design of swirler configuration, a shorter combustor length is possible. Accordingly, to fully realize the potential of the LDI concept, a good understanding of the complex, turbulent swirling flow inside the combustor becomes essential.

To effectively calculate flow fields where swirl, wall effect, flow separation and recirculation are present, a high-level turbulence model should be used. The most widelyused turbulence model in nearly all commercial CFD codes is the $k$ - $\varepsilon$ model. First developed in the 1960s, then popularized in the 1970s, a number of model variations has been published and put into practical use in the CFD community. Examples include the cubic non-linear model [Shih et al., 1998a,b] used in the National Combustion Code (NCC) [Stubbs, 1997; Quealy, 2000], and the standard $k$ - $\varepsilon$ model [Launder and Spalding, 1974] in the KIVA code [Amsden, 1999]. Its popularity is due to the relative ease of implementation and its low CPU and memory overhead. It does, however, have considerable drawbacks for use in resolving flow with strong shear and high gradients of velocity and swirl.

Regardless of the advantages of the $k$ - $\varepsilon$ model, it has a tendency to yield inconsistent and diffusive results for complex flows because of its isotropic nature in modeling eddy viscosity. Without ad-hoc modifications, the $k$ - $\varepsilon$ model often cannot completely describe the large gradients in swirling and recirculating flows. For more accurate calculations and prediction of the complex flow field typical of an engine, or a 
combustor with high degree of swirl, use of a higher-order turbulence model is more appropriate.

Unlike the $k$ - $\varepsilon$ models, the Reynolds-stress turbulence model (RSTM) naturally resolves flow anisotropy, streamline curvature, sudden changes in strain rate, secondary motions, flow in rotation (swirl flow) etc. This is mainly due to the fact that each Reynolds-stress component has its own transport equation. In addition, the production source terms in the RSTM are exact and need not be modeled. This greatly improves the accuracy and robustness of the solver. All in all, the RSTM represents the most comprehensive description of turbulent flows within the framework of the Reynolds averaging approach [Hanjalic, 1999]. Despite the added complexity due to the number of equations in the modeling, the applicability of the RSTM has been made feasible by present-day computer capacity.

In addition to obtaining a better understanding of the complex flow physics inside the combustor, the other purpose of this study is to present cold-flow calculations of the flow field in an LDI combustor with a discrete-jet swirler array using the KIVA-3V code [Amsden, 1999] with an RSTM [Yang et al., 2000]. It also aims to compare the RSTM results with the same calculations by KIVA's built-in standard $k-\varepsilon$ model, and the NCC's cubic non-linear $k-\varepsilon$ model, which has been tuned for swirl flow calculations [Iannetti et al., 2001a,b]. All calculated results are compared to laser-Doppler velocimetry (LDV) data [Jeng et al., 2001] for the same configuration and operating conditions. 


\subsubsection{The National Combustion Code}

The NCC is a research code under development at the NASA Glenn Research Center. It is a 4-stage Runge-Kutta, preconditioned, massively parallel CFD code with super linear scalability [Stubbs, 1997; Quealy, 2000]. The NCC includes various advanced chemistry models, such as the intrinsically low-dimensional manifold and a generalized finite-rate kinetic model. In addition, turbulence-chemistry interaction is modeled via the Magnussen model and a joint-scalar PDF model for turbulent reacting flow. Furthermore, the NCC uses a dynamic wall function for the effect of adverse pressure gradient, a lowReynolds number wall treatment, and unstructured grids for computations. The turbulence models in the code include the standard, quadratic, and cubic $k-\varepsilon$ models.

The cubic non-linear $k-\varepsilon$ turbulence model is a second-order closure-scheme turbulence model, which uses both a non-linear Reynolds stress-strain model [Craft et al., 1993] and a cubic Reynolds stress-strain model [Shih and Lumley, 1993] for mean flow calculations. The model has been tuned and has fewer deficiencies than the standard $k$ - $\varepsilon$ model, particularly in simulating the effects of flow rotation and curvature.

\subsubsection{The KIVA-3V Code with RSTM}

The KIVA-3V computer code has been described in Chapter 4 of this dissertation. To better simulate complex, turbulent engine flow, the Speziale-Sarkar-Gatski (SSG) RSTM [Speziale et al., 1991] has been incorporated into the KIVA code. The SSG RSTM is considered a quasi-linear model, since it contains one quadratic term in the pressurestrain model. One advantage of using the SSG model is that, unlike most other RSTMs available in the literature, no wall-distance parameter is needed to account for wall- 
reflection. This is a desirable feature for engine flow simulations, since the computational domain is often of a complex shape and may also be time dependent. Details of the RSTM formulation, discretization and implementation, numerical issues, and code validation can be found in Chapter 3 and 4, as well as Yang et al. [2000].

\subsubsection{Problem Description and Computational Mesh}

The multi-point LDI combustor has a rectangular inlet section $1.27 \mathrm{~cm}$ long and is upstream of nine groups of swirlers arranged in a 3-by-3 pattern, as shown in Figure 5.3.1. Each group of swirlers comprises eight flow passages, all of which orientated at $35^{\circ}$ in the tangential direction, Figure 5.3.2. Figure 5.3.3 illustrates the 3-by-3 swirler array arrangement and the relative location of the flow passages. A rectangular burner duct, either $7.62 \mathrm{~cm}$ or $17.78 \mathrm{~cm}$ long - depending on the grid density, is downstream of the swirler section. The duct has a cross section of $7.62 \mathrm{~cm} \times 7.62 \mathrm{~cm}$. The inlet surface of the combustor lies in the XY-plane and the inlet velocity is in the positive Z (axial) direction.

The computational grids are constructed by using the Pro-Engineer ${ }^{\circledR}$ software to create a three-dimensional model, which is then exported in IGES format to the Gridgen ${ }^{\circledR}$ [Gridgen] database. A Cartesian multi-blocked structured mesh is then generated using Gridgen. ${ }^{\circledR}$ Next, the grids are exported from Gridgen ${ }^{\circledR}$ into the KIVA code where all blocks are appropriately interconnected. This procedure not only allows for the construction of any complex geometry, but is also efficient in the use of time and resources.

To insure that the calculated results represent a converged solution, a grid dependency study was performed. Three grid densities were chosen for this purpose, 
namely coarse, medium, and dense grids. The grid densities are given in Table 5.5, along with the combustor length used in each computational model. Notice that, for the dense grid case, the rectangular burner duct has a length of $7.62 \mathrm{~cm}$. This length was chosen for CPU time consideration and was based on the LDV measurements. From the LDV data, there is no reversed flow at this location and the measured recirculation bubbles are no more than $5.3 \mathrm{~cm}$ in length. Comparisons of the LDV data with the numerical results among the three computational meshes show that the solution converges with grid density. Therefore, the dense-grid result is chosen for presentation.

Table 5.5: Grid Density

\begin{tabular}{|l|c|c|c|}
\cline { 2 - 4 } \multicolumn{1}{c|}{} & Coarse & Medium & Dense \\
\hline Number of Cells & 357,257 & 527,687 & 965,905 \\
\hline Number of Vertices & 360,450 & 528,618 & 967,312 \\
\hline Burner Length Modeled & $17.78 \mathrm{~cm}$ & $17.78 \mathrm{~cm}$ & $7.62 \mathrm{~cm}$ \\
\hline
\end{tabular}

\subsubsection{Discrete-Jet Swirler Array Flow Physics}

Swirl flow is created when air is forced through the small flow passages, inclined at an angle with respect to the cross-flow plane. As air enters and contracts through the small passages, air velocity increases, producing the so-called discrete jets. As each jet enters the combustor, it spreads, expands, and interacts with the neighboring jets and the bounding walls. Due to the orientation of the flow passages, azimuthal and radial velocity components, which are responsible for the creation of swirl, are developed. When the swirl reaches a certain strength, reversed or recirculating flow regions will form near the top of the combustor. 
Based on the arrangement of the swirler array shown in Figure 5.3.3, the nine groups of swirlers can be classified, according to their geometric locations, into three categories, namely "center," “corner," and "side" [Jeng et al., 2001]. Since the "center" swirler is not bounded by any solid walls but is instead surrounded by other swirlers, it is expected that it will receive the highest level of interactions with other swirlers. Two solid walls and two neighboring swirlers, on the other hand, bound the four "corner" swirlers. The remaining four "side" swirlers have three sides bounded by other swirlers and one side by a solid wall. On the basis of flow physics, the same swirler type is expected to behave similarly. Further discussion of the complex flow pattern inside the combustor will be given in the RESULTS \& DISCUSSION section through the aid of both measured and computed results.

\subsubsection{Flow Conditions and LDV Measurements}

For comparison, the operating conditions in this study are identical to the LDV measurements by Jeng et al. [2001] and an NCC study reported by Iannetti et al. [2001a,b]. The inlet axial velocity is $6.6 \mathrm{~m} / \mathrm{s}$. As in the KIVA code, wall functions are used for all solid walls. At the inlet, the turbulence intensity and length scale are assumed 10\% of the inlet mean flow kinetic energy and $25 \%$ of the inlet opening, respectively. The outlet pressure is at the ambient condition.

The experimental method and assumptions reported in Jeng et al. [2001] are now briefly described. The measurements were made with a two-component LDV system, at twelve axial stations downstream of the discrete-jet swirlers. Due to geometric symmetry of the swirler array, only 4/9 of each cross-section (Figure 5.3.3) was covered during the 
experiment. In other words, the LDV system measured the axial (W) and a cross-flow velocity component $(\mathrm{U})$ within the volume downstream of four neighboring swirlers. The third velocity component, V, was derived by assuming that the flowfield was antisymmetrical about the diagonal.

\subsubsection{Results and Discussion}

The primary objective of this study is to investigate the flow field of an LDI combustor with multipoint swirler array in a square duct, and to compare results among the SSG RSTM, the standard $k-\varepsilon$ model in the KIVA code, the cubic non-linear $k-\varepsilon$ model in the NCC, and the LDV measurements. As the measurements were made in a nonreacting flow, only cold-flow calculations are presented. In the NCC study [Iannetti et al., $2001 \mathrm{~b}$ ], tetrahedral grids were used with a total of 2.26 million cells and 2.28 million vertices. Comparisons of results are presented in several XY-planes, i.e., at $Z=0.3 \mathrm{~cm}$, $1.3 \mathrm{~cm}, 2.3 \mathrm{~cm}$, and $5.3 \mathrm{~cm}$. These cutting-plane locations are shown in Figure 5.3.1. In addition to contour plots, line plots along two axial lines downstream of the swirlers are also presented for quantitative comparison. The first line is located at $\mathrm{X}=2.54 \mathrm{~cm}$, $\mathrm{Y}=2.54 \mathrm{~cm}$ (at the center of the bottom, right corner-swirler), and the second at $\mathrm{X}=1.27 \mathrm{~cm}, \mathrm{Y}=1.27 \mathrm{~cm}$ (between four swirlers at the bottom, right corner).

The results in the XY-planes show that the RSTM yields a significantly better and more consistent agreement with the experiment than do both the $k$ - $\varepsilon$ models. At $\mathrm{Z}=0.3 \mathrm{~cm}$, Figure 5.3.4, although the RSTM (Figure 5.3.4c) calculated a slightly stronger forward velocity (red spots) at the jets, the size, shape and strength of the reversedvelocity contours compare considerably well with the LDV data. The $k-\varepsilon$ models, 
Figures 5.3.4a-b, despite showing acceptable contours of forward velocity, had difficulty computing the reversed flow. In particular, the NCC $k-\varepsilon$ result displays a strong presence of recirculation zone (the blue regions) surrounding the center swirler. This suggests a substantial amount of over-compensation for the diffusiveness inherited in the standard $k$ - $\varepsilon$ formulation.

Further downstream at $Z=1.3 \mathrm{~cm}$, Figure 5.3 .5 contains interesting and discerning differences among the turbulence models. The experimental measurements, Figure 5.3.5d, show kidney-shaped reversed-flow regions around the bounding walls, and a circular region at the center. This is caused by the high shear induced by the interactions among the co-rotating swirlers. The RSTM result, Figure 5.3.5c, agrees very well with the LDV data. The locations and magnitude of the recirculation zones are essentially resolved by the RSTM. The $k-\varepsilon$ models, on the other hand, each computed an inaccurate yet distinct profile. While the standard $k-\varepsilon$ model result, Figure $5.3 .5 \mathrm{~b}$, may seem reasonable, the locations of the reversed-flow regions have shifted. More importantly, the size of these regions is much smaller compared to the LDV plot. This is an indication of the wellknown diffusive nature of the standard $k-\varepsilon$ model. In contrast, the NCC's $k-\varepsilon$ model, Figure 5.3.5a, failed to resolve the recirculation structures. It instead calculated rather well behaved and circular reversed flow regions, without much swirler-swirler interactions. In the NCC studies [Iannetti et al., 2001a,b], it was assumed that the combustor geometry was rotationally periodic every ninety degrees. While this is true in geometric terms, the LDV data in both Figures 5.3.4 and 5.3.5 show that the flow field is not symmetrical about both the X-and Y-axes. Therefore, a periodic boundary condition may not be appropriate for this particular geometry and swirler array configuration. Nevertheless, since the corner 
kidney-shaped recirculation is located away from the symmetry boundaries, the flow structure should be resolved regardless of the symmetry assumption. In addition, some of the NCC-computed recirculation zones appear larger than the measured ones. This again suggests that the cubic non-linear $k$ - $\varepsilon$ model may have been over-adjusted to counteract the diffusiveness found in the standard $k-\varepsilon$ model. Together, the results from both $k-\varepsilon$ models strongly indicate the downside of ad hoc modifications in turbulence modeling. Modifying the two-equation formulation on a case-by-case basis is problematic and therefore should be used with caution, particularly when large gradients, strong shear, flow separation, and high swirl are expected.

While Figure 5.3.5 characterizes the complex flow physics associated with the discrete-jet swirlers, Figure 5.3.6 shows with velocity vectors how the swirling jets interact with each other and with the solid walls along the axial direction $(Z=1.3 \mathrm{~cm}$, $2.3 \mathrm{~cm}$, and $5.3 \mathrm{~cm}$ ). In Figure 5.3.6b, all the "corner" and "side" swirler vortex-centers have shifted relative to each swirler group's center due to wall effect and swirling jet interactions. Smaller, secondary induced vortices due to these interactions are also visible. The center swirler, on the other hand, is able to preserve its characteristics while gaining strength from its neighbors, hence maintaining a nearly circular reversed-flow structure. Further downstream at $Z=2.3 \mathrm{~cm}$, Figure 5.3.6b, due to jet spreading, expanding, and interacting processes and wall effect, those swirlers neighboring the wall gradually merge and eventually form a single, albeit weaker, swirl, Figure 5.3.6c.

The line plots in Figures 5.3.7-10 quantitatively show, in further detail, how the RSTM computations give better agreement with the measurements than do the $k$ - $\varepsilon$ calculations. Since in the LDV experiment two velocity components (U, W) were 
measured, it is appropriate to present comparisons in terms of these components. Figure 5.3.7 contains two line plots of U-velocity comparison among the measured and computed results. The first plot, Figure 5.3.7a, presents the U-velocity along the axial locations downstream of the bottom, right swirler. Immediately below the swirler, the LDV data shows small, negative U-velocity, which quickly becomes positive further downstream, reaches a peak, and gradually tapers off. This implies an axial shift of momentum from the presence of neighboring swirlers. Comparing the LDV measurements with the calculated results provides an obvious distinction among the different turbulence models. The RSTM agrees exceptionally well with the LDV data, while the two $k$ - $\varepsilon$ models give contrasting profiles, particularly before $Z=3.0 \mathrm{~cm}$ where recirculation is strong. At $Z=1.4 \mathrm{~cm}$, the KIVA's standard $k-\varepsilon$ model calculated a peak value approximately $175 \%$ higher than the measurements, while the NCC, unable to resolve the peak, computed much lower values.

Figure 5.3.7b contains a line-plot comparison along axial locations between the four bottom-right swirlers. The RSTM shows a close agreement with the LDV data, with a maximum offset near $Z=1.0 \mathrm{~cm}$. The $k-\varepsilon$ results exhibit similar behavior as in Figure 5.3.7a, except the standard $k-\varepsilon$ model calculated much lower values and the cubic non-linear model, much higher. Both plots in Figure 5.3.7 illustrate the inconsistency of $k-\varepsilon$ models when the flow is complex.

The line plots in Figure 5.3.8 quantitatively present the axial velocity component (W). In Figure 5.3.8a, from the LDV data zero axial-velocity occurs at approximately $\mathrm{Z}=2.2 \mathrm{~cm}$ downstream of the swirler, which may be assumed to be the size of the recirculation bubble. The RSTM profile closely follows the LDV data, although a 
maximum offset of $25 \%$ occurs below the recirculation region. The NCC's $k$ - $\varepsilon$ model computed a bubble size nearly twice the measured one, while KIVA's $k$ - $\varepsilon$ model calculated only half, clearly displaying its diffusive nature. Figure $5.3 .8 \mathrm{~b}$, on the other hand, does not show as distinct a comparison as does Figure 5.3.8a. The RSTM result exhibits the general trend of the LDV profile for $Z<1.0 \mathrm{~cm}$ as well as $Z>3.0 \mathrm{~cm}$. The NCC calculates a substantially stronger reversed flow in the $\mathrm{Z}<1.0 \mathrm{~cm}$ region.

Although the V-velocity component was not directly measured in the LDV experiment, it would nevertheless be useful to show the line plots of swirl velocity and turbulent kinetic energy, $k$. The plots that follow, therefore, serve as comparisons of trend rather than accuracy. The swirl velocity in Figure 5.3.9a carries a characteristic similar to the U-velocity plot in Figure 5.3.7a. The swirl quickly peaks at about $Z=1.4 \mathrm{~cm}$, then gradually settles. The RSTM basically follows the trend and stays within $20 \%$ of the measured values. The highest swirl computed by the standard $k$ - $\varepsilon$ model occurs at a $Z$ value close to the measured one, and the cubic non-linear $k$ - $\varepsilon$ model was unable to resolve any swirl structure within the recirculation zone.

Figure $5.3 .9 \mathrm{~b}$ shows a more complex profile, particularly for $\mathrm{Z}<3.0 \mathrm{~cm}$. The measured swirl values are generally lower than those in Figure 5.3.9a, and fluctuate considerably inside the bubble. Both $k-\varepsilon$ models generally yield excessive swirl up to $\mathrm{Z}=2.5 \mathrm{~cm}$. Halfway downstream and onward, however, all computed results agree quite well with the LDV measurements.

The last set of figures contains the $k$ levels at the same locations as in the preceding line plots. Figure 5.3.10a shows that the NCC's $k-\varepsilon$ model fails to capture the steep profile of the measurements, near $Z=1.5 \mathrm{~cm}$. Instead, the model gives a generally flat line that 
falls below the LDV data. Since eddy viscosity, $\mu_{\mathrm{T}}$, is directly proportional to the square of $k$, a small $k$ value results in an even smaller $\mu_{\mathrm{T}}$. By the same token, the standard $k$ - $\varepsilon$ model, having $k$ values much higher than the LDV data, has excessively large $\mu_{\mathrm{T}}$ throughout the entire combustor length. In Figure 5.3.10b, all calculated results fall below the LDV data, albeit exhibiting similar trend.

\subsubsection{Conclusion}

An LDI combustor with a discrete-jet swirler array was modeled for cold-flow investigation. The complex flow physics associated with the swirler array was described with the aid of CFD results and experimental data. The SSG RSTM was used to describe the turbulence inside the combustor. The RSTM-computed results were compared to LDV measurements, as well as results from the cubic non-linear $k-\varepsilon$ turbulence model in the NCC, and the standard $k-\varepsilon$ model in the KIVA code. The result comparisons show that the RSTM accurately captured most of the flow characteristics. Both $k-\varepsilon$ models exhibited contrasting behaviors, either over- or under-predicting eddy viscosity in regions of high swirl and strong recirculation. Similar conclusion was also reached in a previous study of an LDI combustor with an array of axial swirlers [Yang et al., 2003a].

Accurate calculation of swirl and recirculation zones is important in deciding fuel spray locations as well as in the design of combustors. Within the Reynolds averaging approach, the RSTM has proven to be a proper choice for accurately predicting complex flow characteristics, given the general capacity and availability of current-generation computers. The RSTM computations required 178.4 msec per cycle per grid point on an SGI Origin 3400 platform, while the computation with KIVA's $k$ - $\varepsilon$ model took $87.6 \mathrm{msec}$. 
The extra computational overhead from using the RSTM is justified by its accuracy and robustness. No ad hoc modifications are necessary for complex geometry and flow conditions. Future work may include study of similar combustor geometry with different swirl angles as well as with combustion and fuel spray. 


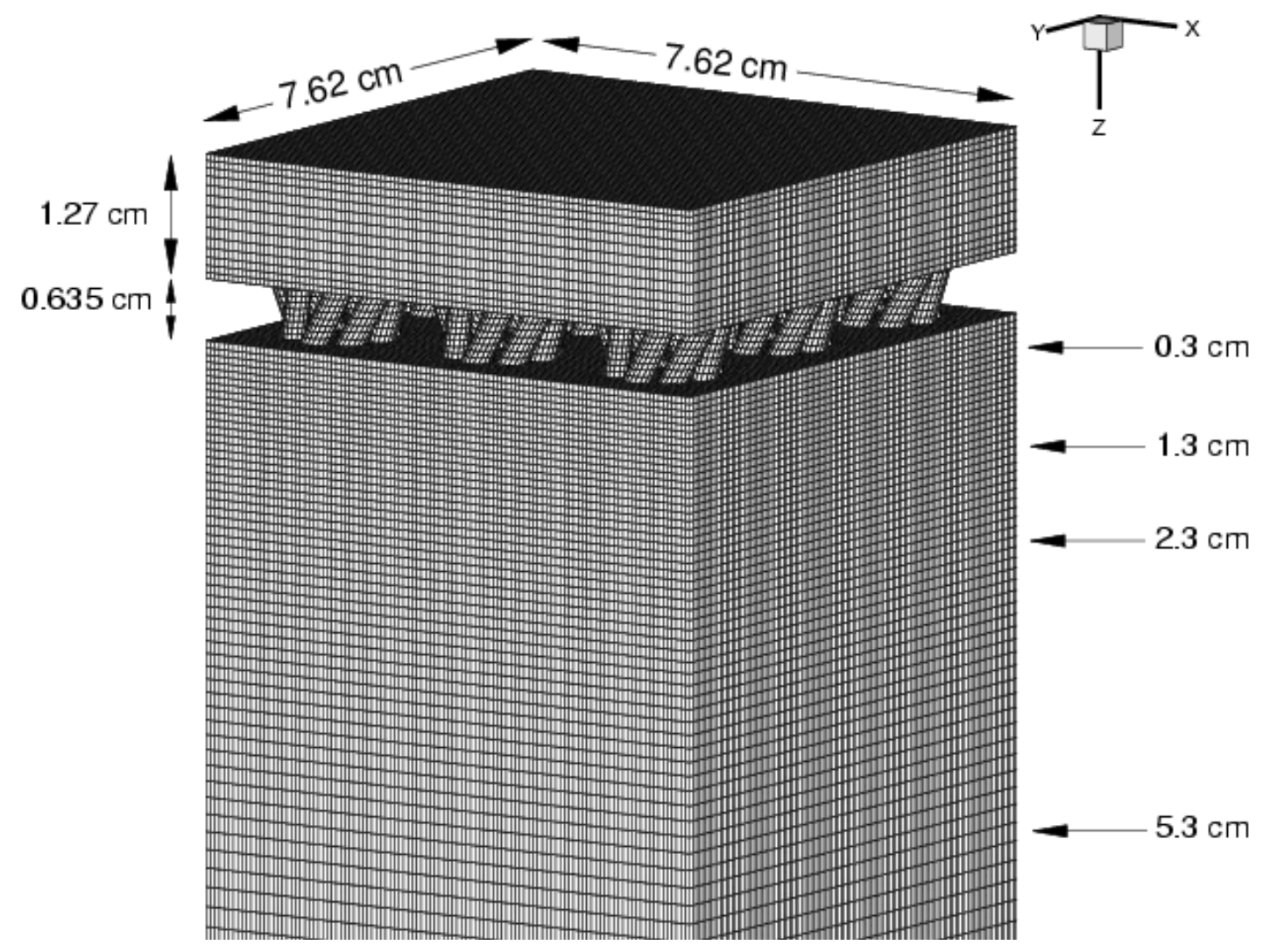

Figure 5.3.1. Combustor Mesh and Dimensions

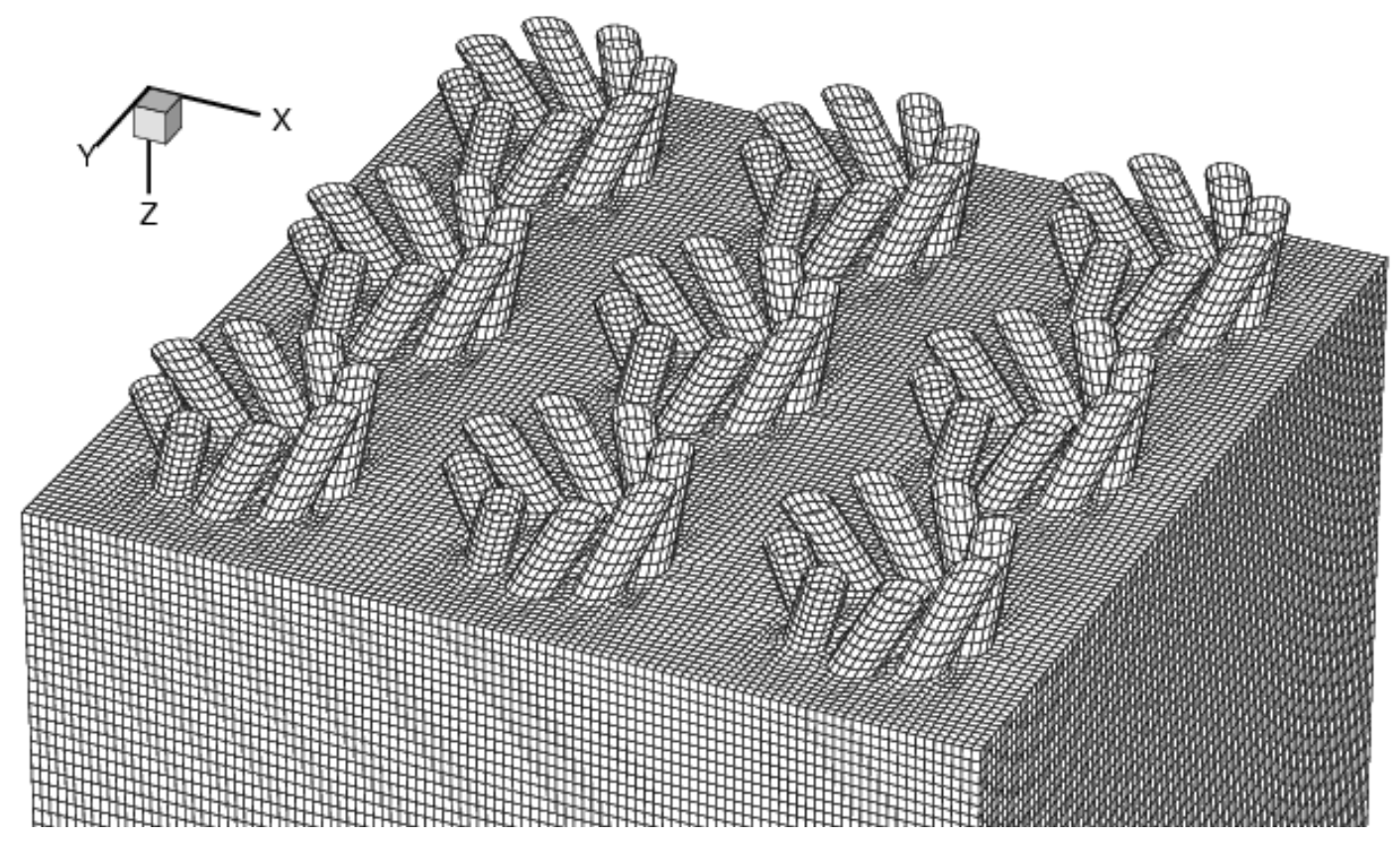

Figure 5.3.2. Discrete-Jet Closeup 

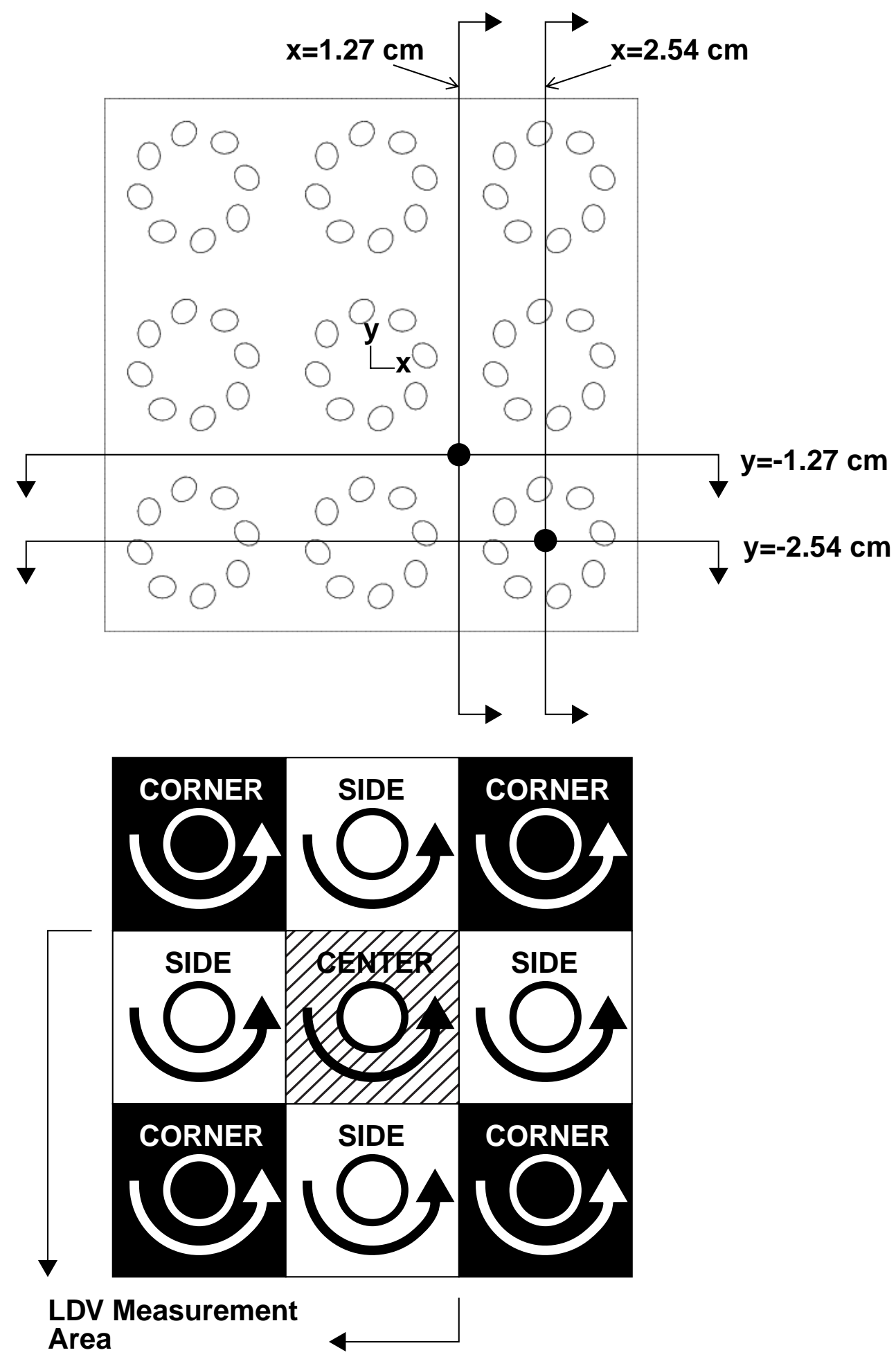

Figure 5.3.3. Line-plot Data Locations; Swirler Array Configuration and Classification 


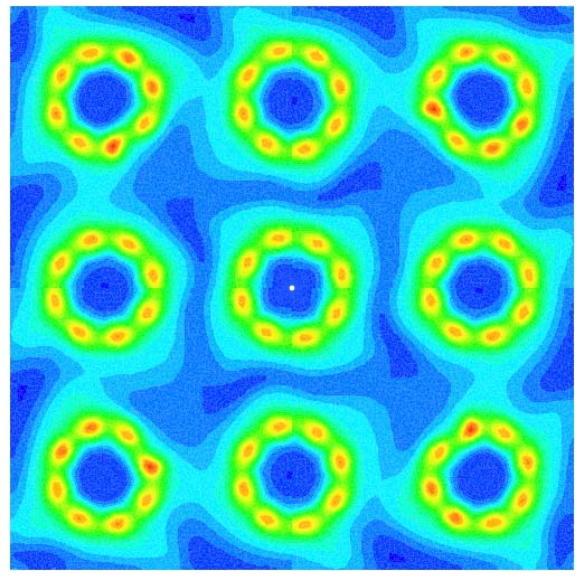

(a) NCC $k-\varepsilon$ Model

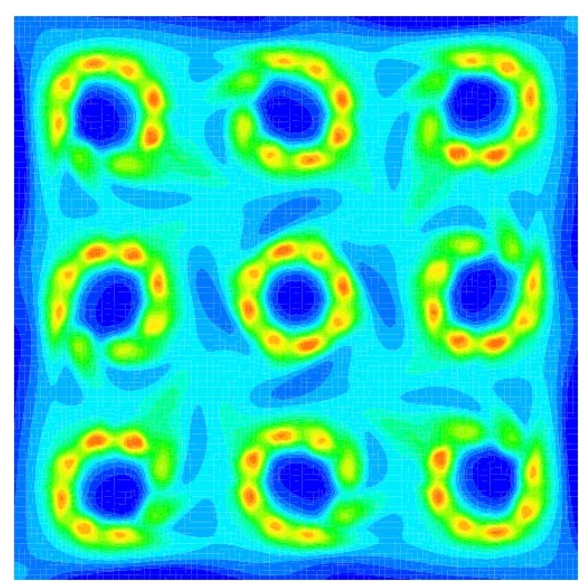

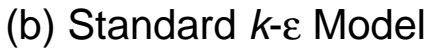

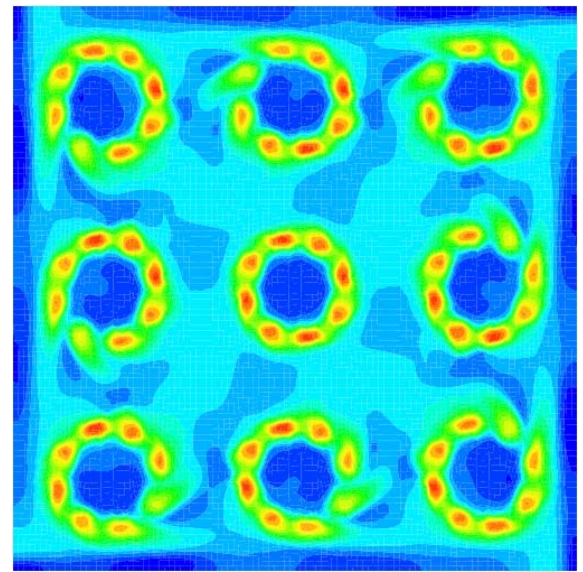

(c) Reynolds-Stress Model

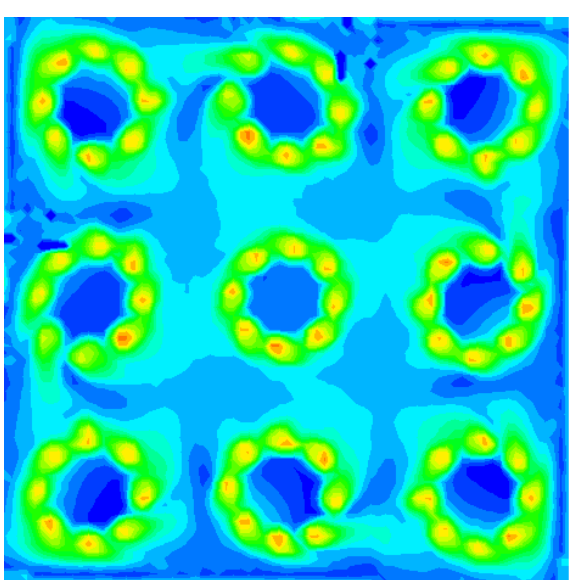

(d) LDV Measurements

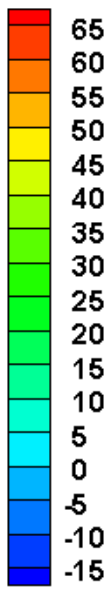

15

Figure 5.3.4. Axial Velocity (W) Contours at $Z=0.3 \mathrm{~cm}$ 


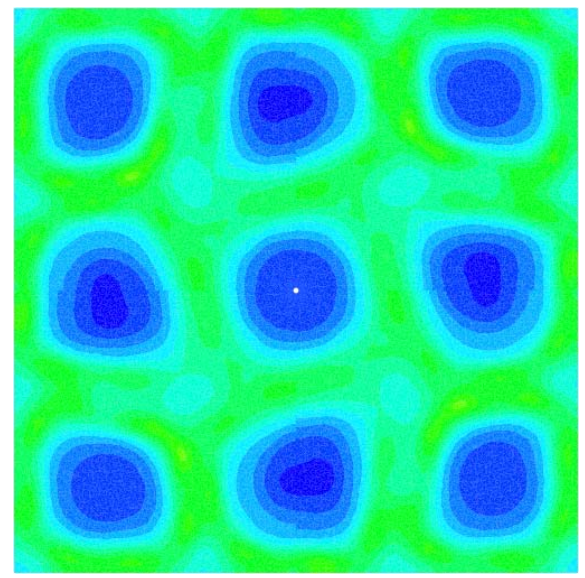

(a) NCC $k-\varepsilon$ Model

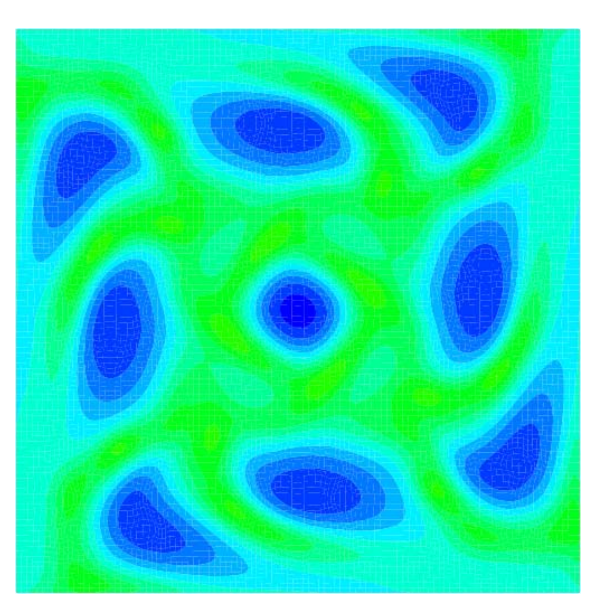

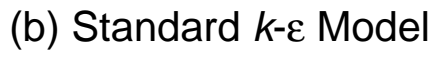

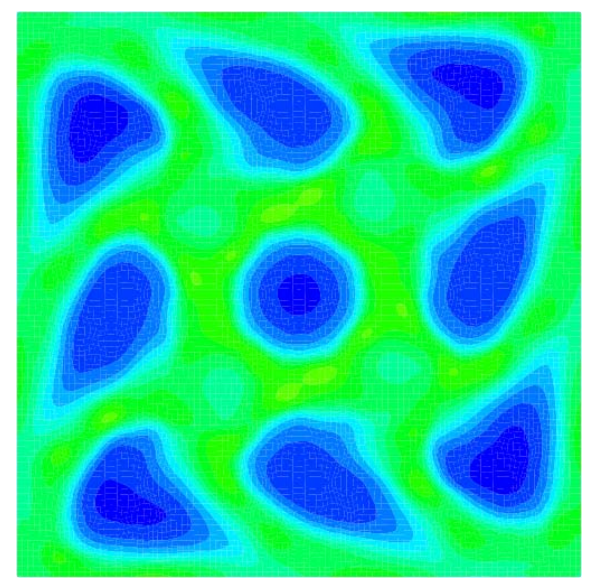

(c) Reynolds-Stress Model

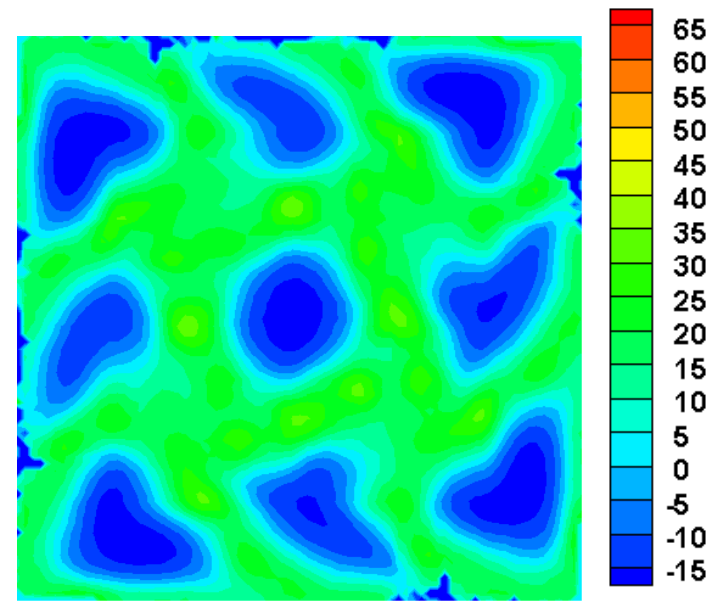

(d) LDV Measurements

Figure 5.3.5. Axial Velocity (W) Contours at $Z=1.3 \mathrm{~cm}$ 


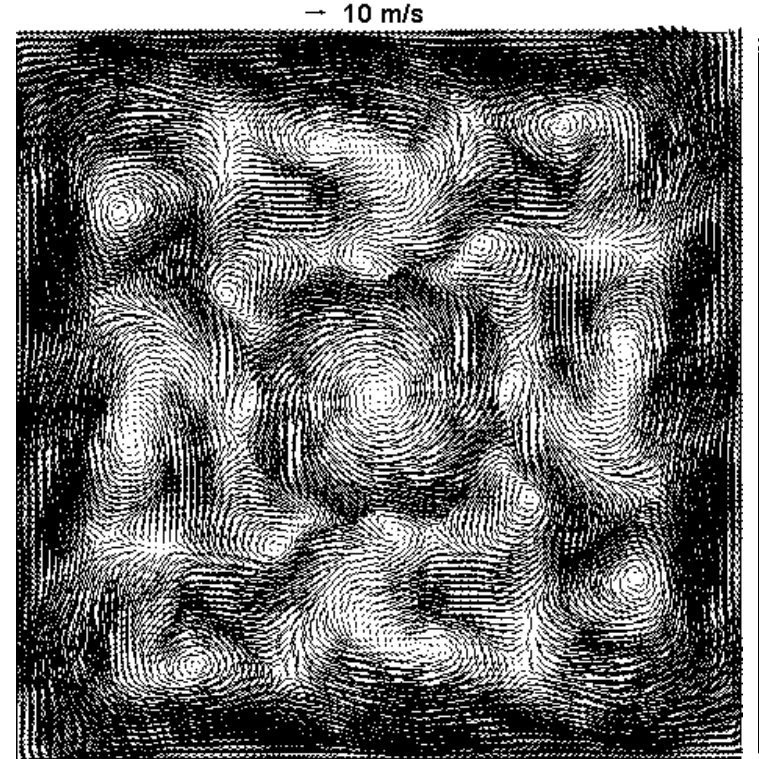

(a) $Z=1.3 \mathrm{~cm}$ $\rightarrow 10 \mathrm{~m} / \mathrm{s}$

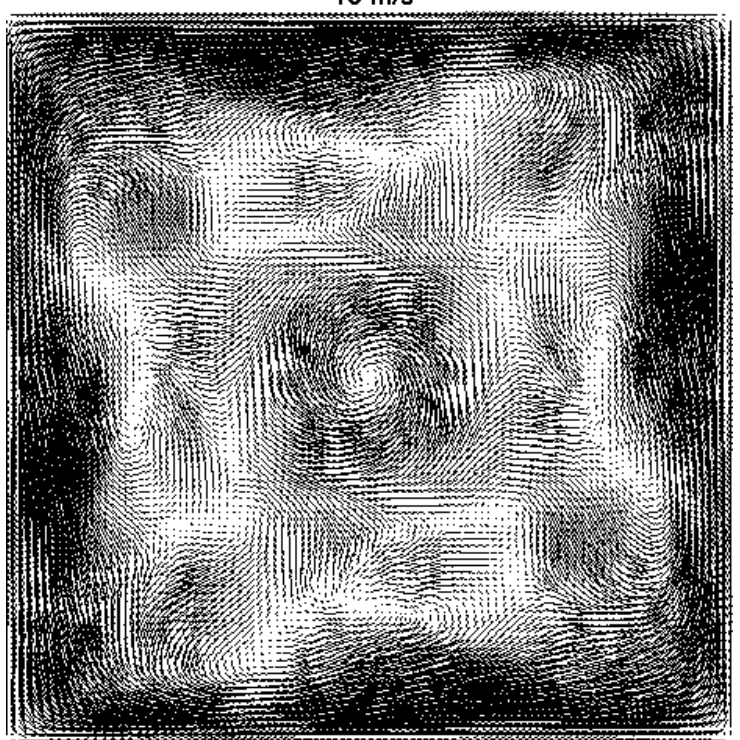

(b) $Z=2.3 \mathrm{~cm}$

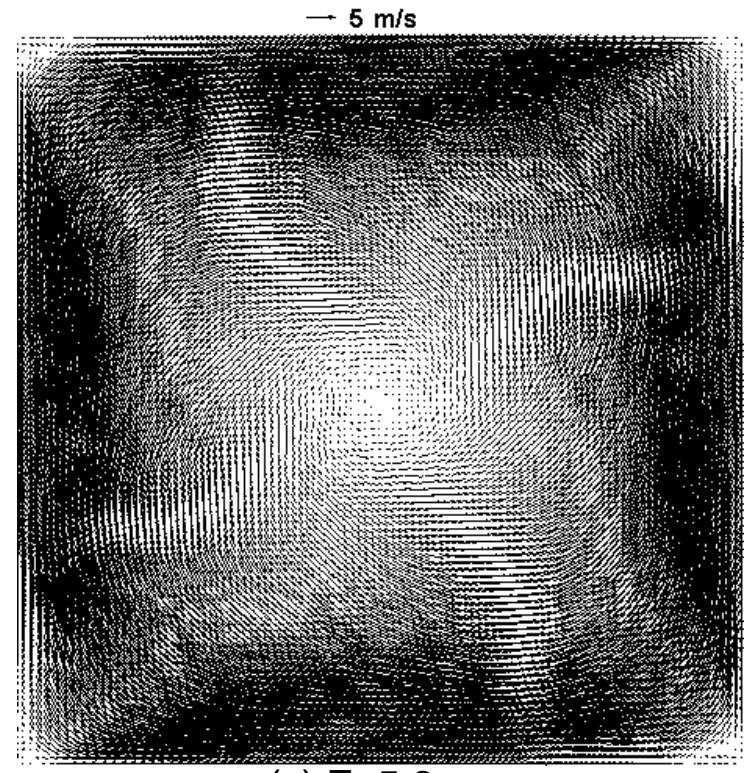

(c) $Z=5.3 \mathrm{~cm}$

Figure 5.3.6. UV-Velocity Vectors at Various XY-Planes, RSTM Results 


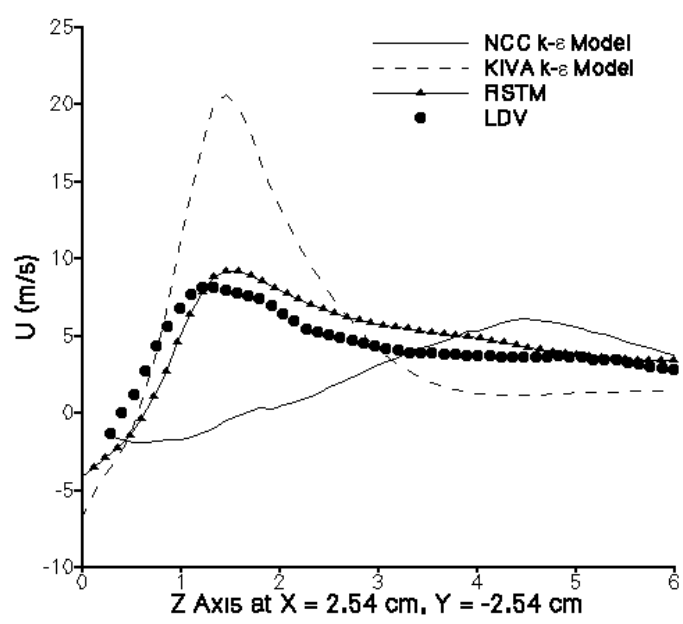

(a)

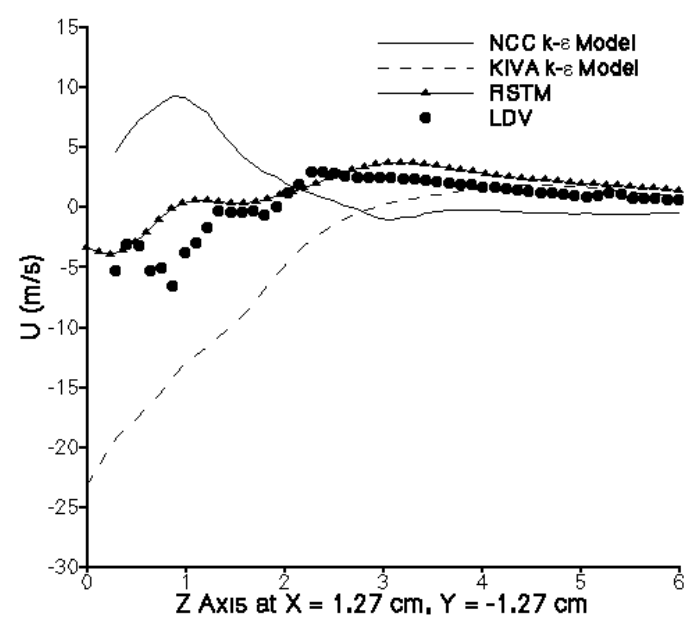

(b)

Figure 5.3.7. Line-Plot: U-Velocity Component

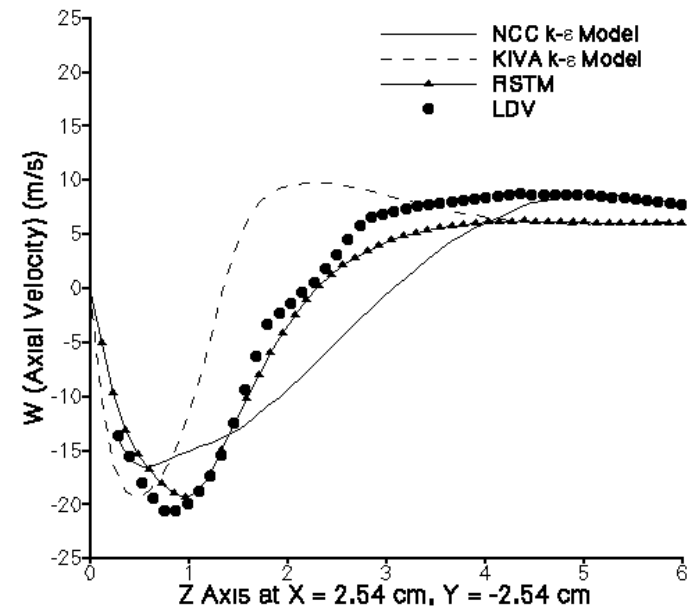

(a)

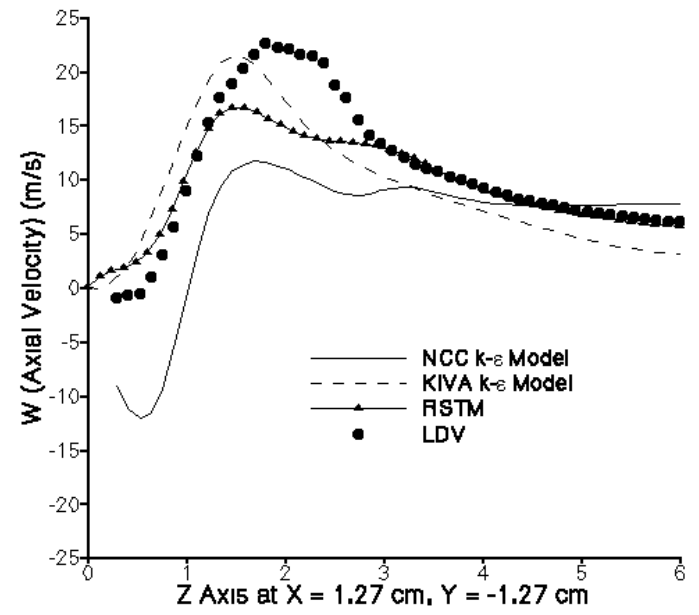

(b)

Figure 5.3.8. Line-Plot: W-Velocity (Axial) Component 


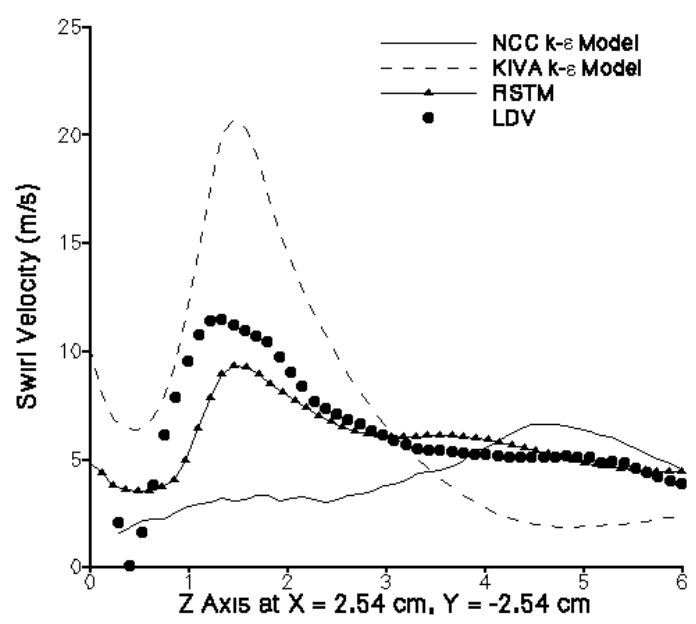

(a)

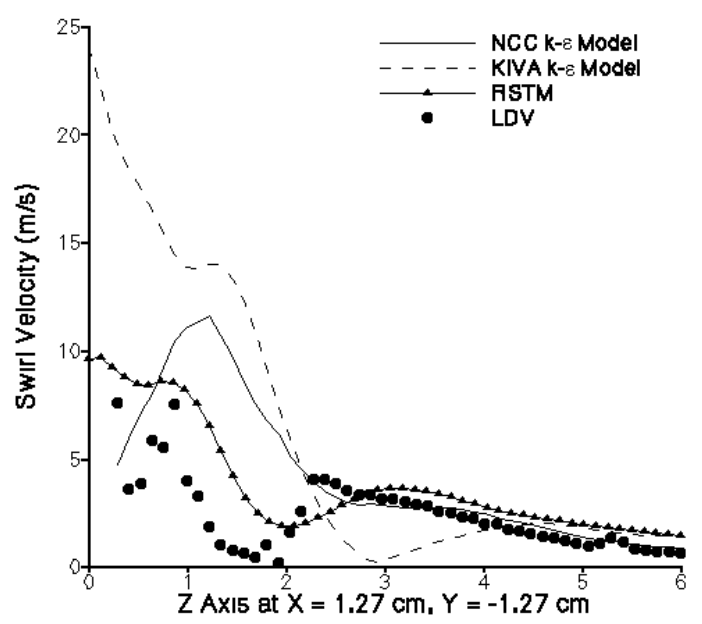

(b)

Figure 5.3.9. Line-Plot: Swirl Velocity

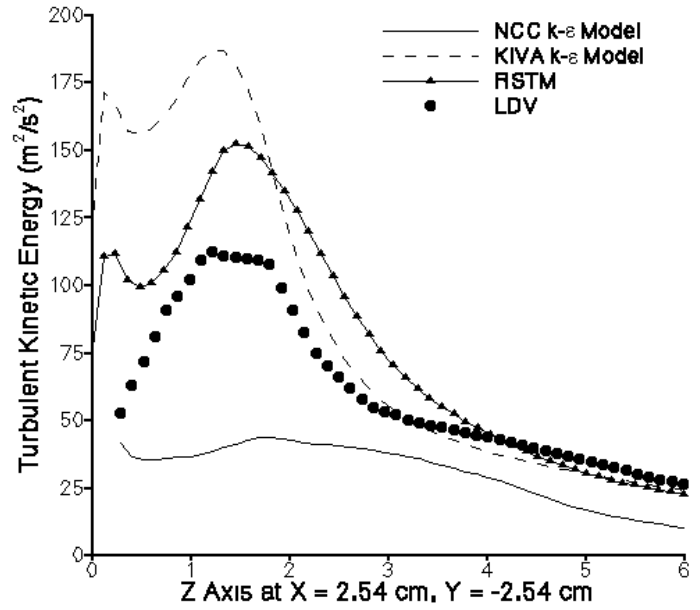

(a)

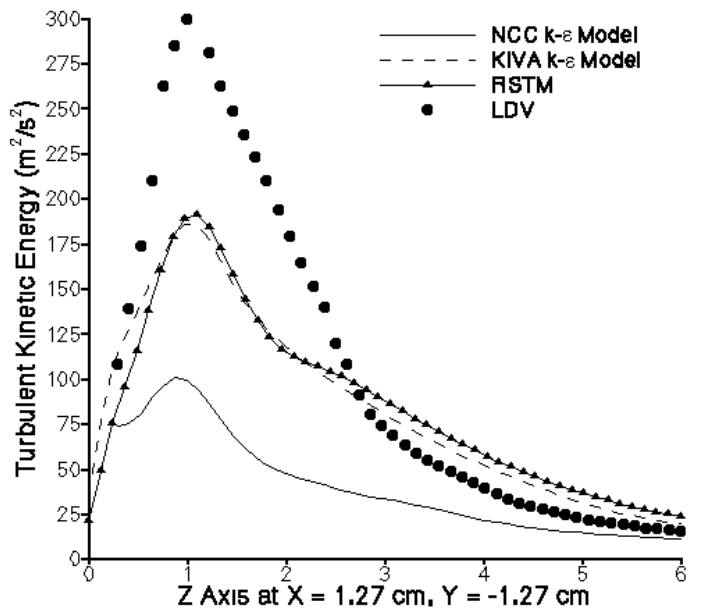

(b)

Figure 5.3.10. Line-Plot: Turbulent Kinetic Energy 


\subsection{Engine Flow Simulation}

To properly simulate the highly anisotropic turbulent engine flows, higher order turbulence model should be used to correctly reproduce flow physics inside the engine. The popular KIVA computer code has been modified to include the Reynolds-stress turbulence model (RSTM) for this purpose. The objective of this study is to present our recent research on the use of RSTM and the KIVA code for engine flow simulation, which include gas turbine combustors and IC engines.

\subsubsection{Introduction}

Fluid flows in engines are always turbulent and are highly anisotropic due to complex geometry, wall effects, flow rotation (swirl), internal separation, etc. For internal combustion (IC) engine flow, additional complications are involved because of the rapid compression and expansion strokes produced by the piston motion. Among these flow phenomena, swirl is an essential and desirable engine design feature. Modern gas turbine combustors use strong swirl to improve combustor performance by aiding in the fuel-air mixing process and by producing recirculation regions which can act as flame holders for flame stability [Lefebvre, 1983]. For direct-injection IC engines, swirl is used to enhance the air fuel mixing for emission control and better efficiency. Therefore, to properly simulate flow field inside various types of engines, the swirl characteristic needs to be resolved by a robust and versatile turbulence model, without having to perform ad hoc modifications for each engine geometry. 
Most industrial computations of engine flows are performed using two-equation models, such as the $k-\varepsilon$ or the $k$ - $\omega$ model, in which the Boussinesq approximation is used to relate the Reynolds stress tensor $\left(u_{i} u_{j}\right)$ to the mean flow strain rate $\left(S_{i j}\right)$, i.e.,

$$
u_{i} u_{j} \propto S_{i j}
$$

The proportionality coefficient, i.e., the eddy viscosity $\left(\mu_{T}\right)$, in the $k$ - $\varepsilon$ model is expressed in terms of fluid mean density ( $\rho$ ), turbulence kinetic energy $(k)$, and its dissipation rate

$$
\mu_{T} \propto \frac{\rho k^{2}}{\varepsilon}
$$

Since $\rho, k$, and $\varepsilon$ are all scalars, accordingly, the eddy viscosity is isotropic in contrast to real situation in complex flows. The Reynolds-stress (second-moment) turbulence model (RSTM), on the other hand, does not suffer from this shortcoming.

Within the framework of the Reynolds averaging approach, the RSTM represents the most comprehensive description of turbulent flows, and can be employed for practical computations with the present-generation workstations [Hanjalic, 1999]. One major advantage of RSTM is that the source terms are exact and need not be modeled. This becomes especially important in flows with complex strain field where in addition to simple shear, dilation and compression strain are significant [Hanjalic, 1999]. Since each Reynolds-stress component is described by a transport equation, the RSTM naturally includes effects of anisotropy, streamline curvature, sudden changes in strain rate, secondary motions, flow rotation.

Two RSTMs, namely the LRR [Launder et al., 1975] and the SSG [Speziale et al., 1991] models, were implemented into the popular KIVA [Amsden, 1999] family computer 
codes. The LRR model, especially its IP version, is often referred to as the standard RSTM, characterized by the simple linear models of the pressure-strain redistribution process, but for the near-wall flows it requires the inclusion of a wall-reflection term (not accounted for here). The SSG model is considered a quasi-linear model since it contains one quadratic term in the pressure-strain model. One advantage of using the SSG model is that, unlike most other RSTMs available in the literature, no wall-distance parameter for accounting for wall-reflection term is needed. This is a desirable feature for reciprocating engine flow simulations, since the computational domain is of a complex shape and changes constantly in time due to piston motion.

The objective of this study is to present recent studies on the use of the RSTM and KIVA code for engine flow simulation. The results to be presented include (1) multi-point lean direct injection (LDI) gas turbine combustor with discrete-jet swirlers, (2) multi-point LDI gas turbine combustor with helical axial swirlers, (3) a simplified direct injection stratified charged (DISC) IC engine, (4) a lean-premixed prevaporized (LPP) combustor, and (5) a four-valve DOHC (Double Overhead Camshaft) IC engine. Due to space limitation, the Reynolds-stress modeling equations, details of the KIVA code implementation, and code validation will not be presented here, but can be found in Chapter 3 and 4 of this dissertation, as well as Yang et al. [2000].

\subsubsection{Results and Discussion}

\section{LDI Combustor with Discrete-Jet Swirlers}

The multi-point LDI combustor has a rectangular inlet section $12.7 \mathrm{~mm}$ long (Figure 5.4.1), and is upstream of nine groups of swirlers arranged in a $3 \times 3$ pattern 
(Figure 5.4.2). Each group of swirlers comprises eight passages, all co-rotated at $35^{\circ}$ in the tangential direction (Figure 5.4.3). A 177.8 or $76.2 \mathrm{~mm}$-long rectangular burner duct is downstream of the swirler section. The combustor has a cross section of $76.2 \mathrm{~mm}{ }^{\prime} 76.2$ mm. The inlet surface of the combustor lies in the $\mathrm{X} Y$ plane and the inlet velocity is specified to be in the axial or positive Z-direction. The final Cartesian computational mesh shown in Figure 5.4.1 has a total of 965,905 cells and 967,312 vertices.

For comparison, the primary flow conditions were identical to the parameters in a report by Iannetti et al. [2001b] in which the NASA NCC (National Combustor Code) [Liu and Quealy, 1998] was used along with a cubic nonlinear $k-\varepsilon$ model tuned for swirl flow calculation [Shih et al., 1998b]. The inlet axial velocity was $6.6 \mathrm{~m} / \mathrm{s}$. Law-of-wall was applied on all solid walls. In addition, the turbulence intensity and length scale were assumed $10 \%$ and $3 \%$, respectively, at the inlet. The exit temperature and pressure were at the ambient condition.

The primary goal of this study is to investigate the non-reacting flow field of a multi-point injector in a square duct, and to compare results of the SSG model with the cubic nonlinear $k-\varepsilon$ model in NCC and the LDV measurements from an experimental study. As the measurements were made in a non-reacting flow, only cold-flow calculations are presented. The LDV data are taken from a report by Jeng et al. [2001] and the NCC results from Iannetti et al. [2001b]. In the report, tetrahedral grids were used with 2.26 million cells and 2.28 million vertices for a quarter domain of the combustor. Comparisons of results are presented in color contours, in both $\mathrm{X}-\mathrm{Y}$ and $\mathrm{Y}-\mathrm{Z}$ planes, i.e., at $\mathrm{Z}=3 \mathrm{~mm}$, $13 \mathrm{~mm}$ and $23 \mathrm{~mm}$, as well as $\mathrm{X}=19 \mathrm{~mm}$ (between swirlers). These cutting-plane locations are shown in Figure 5.4.1. 
In general, the results in the X-Y planes show that the RSTM yields a better agreement with the experiment than the NCC $k-\varepsilon k-\varepsilon$ calculations. At $Z=3 \mathrm{~mm}$ (Figure 5.4.4), although the magnitude of the forward velocity contours compares fairly well between the measured and both computed results, the NCC-calculated reverse-flow structures differ substantially. In Figure 5.4.4a, the dark blue contours surrounding the center swirlers represent large areas of strong recirculation, which are not present in both Figures 5.4.4b (RSTM) and 5.4.4c (LDV). The RSTM result, Figure 5.4.4b, agrees quite well with the measurement, particularly the forward-flow regions directly below each swirler.

Further downstream, at $Z=13 \mathrm{~mm}$ (Figure 5.4.5), the NCC computation shows circular recirculation zones below the center of the swirlers, where both the RSTM and the experimental measurements show kidney-shaped reverse-flow regions. In Iannetti et al. [2001b], it was assumed that the combustor geometry was rotationally periodic every ninety degrees. While this is true in geometric terms, the LDV data in both Figures 5.4.4 and 5.4.5 show that the flow field is not symmetrical about both the $\mathrm{X}$ - and Y-axes. This is caused by the flow-field structure induced by the interactions among the co-rotating swirlers. Therefore, a periodic boundary condition may not be appropriate for this geometry and swirler configuration.

The line plots in Figures 5.4.6 and 5.4.7 show quantitative comparisons between the measurements and computations. Since in the LDV experiment two velocity components, $\mathrm{U}$ (cross-flow) and $\mathrm{W}$ (axial), were measured, it is appropriate to present comparisons in terms of these components. Figure 5.4.6a contains line plots of U-velocity 
comparison among the measured and computed results, while Figure 5.4.6b shows the Wvelocity comparison.

Figure 5.4.6a-I presents the U-velocity at axial locations downstream of the bottom, right swirler (Location I in Figure 5.4.1). Observing the LDV data, its trend shows an axial shift of momentum from the presence of neighboring swirlers. Such behavior is successfully captured by the RSTM, while the NCC $k-\varepsilon$ model, unable to resolve the peak, computed much lower values, particularly before $\mathrm{Z}=3.0 \mathrm{~cm}$ where recirculation is strong. Figure 5.4.6a-II contains a line-plot comparison of the U-velocity along axial locations between the four bottom-right swirlers (Location II in Figure 5.4.1). The RSTM shows a close agreement with the LDV data, with a maximum offset near $Z=1.0 \mathrm{~cm}$.

Figures 5.4.6b-I and II quantitatively present the axial velocity component (W), at the two locations as in Figure 5.4.6a. In Figure 5.4.6b I, from the LDV data, zero axialvelocity occurs at approximately $Z=2.2 \mathrm{~cm}$ downstream of the swirler, which may be assumed to be the size of the recirculation bubble. The RSTM profile closely follows the LDV data, although a maximum offset of $25 \%$ occurs below the recirculation region. The NCC's $k$ - $\varepsilon$ model computed a bubble size nearly twice the measured one. Figure 5.4.6b-II, on the other hand, does not show as distinct a comparison as does Figure 5.4.6b-I. The RSTM result exhibits the general trend of the LDV profile for $\mathrm{Z}<1.0 \mathrm{~cm}$ as well as $\mathrm{Z}>3.0 \mathrm{~cm}$. The NCC calculates a substantially stronger reversed flow in the $\mathrm{Z}<1.0 \mathrm{~cm}$ region.

Although the V-velocity component was not directly measured in the LDV experiment, it would still be useful to show the line plots of swirl velocity and turbulence kinetic energy, $\mathrm{k}$. The plots that follow, therefore, serve as comparisons of trend rather 
than accuracy. The swirl velocity in Figure 5.4.7a-I carries a characteristic similar to the U-velocity plot in Figure 5.4.6a-I. The swirl quickly peaks at about $\mathrm{Z}=1.4 \mathrm{~cm}$, then gradually settles. The RSTM basically follows the trend and stays within $20 \%$ of the measured values. The cubic non-linear $k$ - $\varepsilon$ model was unable to resolve any swirl structure within the recirculation zone. Figure 5.4.7a-II shows a more complex profile, particularly for $Z<3.0 \mathrm{~cm}$. The measured swirl values are generally lower than those in Figure 5.4.7a-I, and fluctuate considerably inside the bubble. The $k$ - $\varepsilon$ model generally yields excessive swirl up to $Z=2.5 \mathrm{~cm}$. Halfway downstream and onward, however, all computed results agree quite well with the LDV measurements.

Figure 5.4.7b-I shows that the NCC's $k$ - $\varepsilon$ model fails to capture the steep profile of the measurements, near $Z=1.5 \mathrm{~cm}$. Instead, the model gives a generally flat line that falls below the LDV data. Since eddy viscosity, $\mu_{\mathrm{T}}$, is directly proportional to the square of $k$, a small $k$ value results in an even smaller $\mu_{\mathrm{T}}$. In Figure 5.4.7b-II, all calculated results fall below the LDV data while exhibiting similar trend.

Details of this study can be found in Teo et al. [2001].

\section{$\underline{\text { LDI Combustor with Helical Axial Swirlers }}$}

Configuration of this case is similar to the previous one, except that helical axial swirler is used. The modeled combustor includes nine axial swirlers with venturi, which are attached to a single primary combustion zone, Figure 5.4.8. The helical axial swirlers are arranged in a $3 \times 3$ square and lies in the $\mathrm{X}-\mathrm{Y}$ plane. The rotation imparted to the air by each swirler is directed in the axial or Z-direction. Two different swirlers are used based on blade angle inclination from the direction of airflow. One swirler has a $45^{\circ}$ angle, and the other $60^{\circ}$. These angles refer to the outermost vane angle of the helical swirler. A plot 
of a $60^{\circ}$ swirler attached to a convergent-divergent venturi is shown in Figure 5.4.9. The central hollow region is reserved for the fuel injector while the five helical hollow slots represent the blades that guide the airflow.

The computational domain begins with the inlets placed at the top of nine $45^{\circ}$ or $60^{\circ}$ swirlers. These swirlers are co-rotating clockwise. After air exits a swirler it passes through a venturi. A short divergent cone, acting like a diffuser, follows and ends at the primary zone inlet plane. One constant-Y plane and two constant-Z planes are defined for postprocessing, where $\mathrm{Z}$ represents the axial direction. These cutting plane locations are also shown in Figure 5.4.8.

Notable boundary conditions include the wall and inlet boundaries. Since the turbulence equations presented are valid only for high-Re number flows, wall functions were used for wall boundaries. Diffusion fluxes were set to zero at the walls. The specified inlet velocity value depends on the chosen operating conditions of the combustors. These conditions include a pressure drop, $\Delta \mathrm{p}$, of $3 \%$ across the height of the combustor, an outlet pressure of $1.0 \mathrm{~atm}$, and an inlet temperature of $27^{\circ} \mathrm{C}$. These quantities were then used to determine the inlet air velocity.

Calculations were performed with the SSG RSTM and the standard $k$ - $\varepsilon$ model using the quasi-second order upwind scheme in the KIVA code. The computed results were compared with the experimental data for the $60^{\circ}$ co rotating case. In addition, a $45^{\circ}$ co-rotating was also investigated.

The experiments were conducted at the NASA Glenn Research Center. A square duct of quartz walls was used for the primary combustion zone for optical access. The sides of the square were $76.2 \mathrm{~mm}$ wide. A phase/Doppler particle analyzer was used for all 
velocity measurements. A two-component system using green $(514.5 \mathrm{~nm})$ and blue $(488.0$ $\mathrm{nm}$ ) beams from an argon-ion laser operating at $1.5 \mathrm{~W}$ power output was used for the measurements. The transmitting optics utilized a $500 \mathrm{~mm}$ focal length lens combined with a $300 \mathrm{~mm}$ focal length collimating lens to yield a focused beam waist of $131 \mathrm{~mm}$ for the green and $124 \mathrm{~mm}$ for the blue lines. The fringe spacing was $6.788 \mathrm{~mm}$ for the green and $6.667 \mathrm{~mm}$ for the blue lines. The receiving optics were located 30 degrees off-axis in the forward-scatter direction. Light was collected using a $500 \mathrm{~mm}$ focal-length $\mathrm{f} 5.4$ lens and then focused onto a $100 \mathrm{~mm}$ by $1 \mathrm{~mm}$ long slit. Further details of the instrument can be found in a report by Bachalo and Houser [1984], and details of the experimental procedure can be found in Bulzan [1995].

(a). $60^{\circ}$ Co-rotating Swirlers Case

Observations of the results on the $\mathrm{X}-\mathrm{Y}$ planes in Figures 5.4.10 and 5.4.11 show that the RSTM compares more closely to the experiments than does the $k-\varepsilon$ model. These plots display computed results at $\mathrm{Z}=5 \mathrm{~mm}$ and $18 \mathrm{~mm}$, while the experimental plots show the data measurements at the upper right corner of the same X-Y planes. A coordinate system is displayed on the figures to indicate the $\mathrm{X}$ and $\mathrm{Y}$ directions. The positive $\mathrm{Z}$ direction is directed from the swirler inlet to combustor outlet and is implied to be directed into the page.

Figure 5.4.10 paints a distinct picture that demonstrates the accuracy of the RSTM when comparing its result with the $k-\varepsilon$ and experimental plots. Between the two turbulence models, the magnitude of the velocities differ greatly, although the axial velocity (w) contour structures and the vector orientations display similarities. Comparison with the experimental data shows that the reversed flow at the center of the 
circles is better captured by the RSTM. The contour legend shows that the RSTM velocity levels vary over a greater range as seen in the experimental data plot.

At $Z=18 \mathrm{~mm}$ surface in Figure 5.4.11, the RSTM result shows that the forward flow between swirlers and reversed velocity magnitude at the swirler centers are in good agreement with experiment. This shows that the RSTM can better predict the reversed flow associated with recirculations. It also shows its ability to resolve the effects of strong swirl as verified by the experimental data.

Additional observations can be made from Figures 5.4.10 and 5.4.11. The four sub-regions enveloped by any four neighboring circles contain negative axial velocities (i.e., reversed flow) in the RSTM results, whereas the $k$ - $\varepsilon$ plots present little (Figure 5.4.10b) and no (Figure 5.4.11b) reversed flow in these regions. Furthermore, these four sub-areas contain swirls in the counter-clockwise (ccw) direction, opposite to the primary flow direction from the nine swirlers. This is due to the opposing velocities surrounding the central circle, where the shear force in one direction impedes and steers away the shear force in the other, creating the four counter-clockwise swirls.

The contrast between the two models can be further displayed by viewing the velocity on the $\mathrm{X}-\mathrm{Z}$ planes, such as along the $\mathrm{Y}=0.0 \mathrm{~mm}$ cutting plane shown in Figure 5.4.12. Reversed flow is much stronger below the swirlers with the RSTM. For example, the RSTM reversed flow reaches up to the venturi while the $k$ - $\varepsilon$ result falls far short. Concurrently, the forward flow regions predicted by the RSTM are also stronger. Since much of the cross section of the combustor has a reversed mass flux, the forward mass flux regions must be stronger to satisfy overall mass conservation. 
The $k$ - $\varepsilon$ model results appear more diffusive than those of the RSTM. This is due to the overestimated eddy viscosity which is a known characteristic of the standard $k$ - $\varepsilon$ model. This set of results also indicates that without ad hoc modification, the $k-\varepsilon$ model is simply unable to capture the essence of flow recirculation from locally large axial pressure gradients created by the high degree of swirl [Gupta et al., 1984]. As air exits from a swirler into the combustor, its swirling motion creates strong pressure gradients in the radial direction, a phenomenon similar to a free swirling jet being ejected from a nozzle. As the flow carries itself further downstream, an adverse pressure gradient builds up within the boundary of the air stream, causing the flow to reverse its axial direction and hence, a recirculation zone is created downstream of each swirler.

In short, with the RSTM whose Re-stress components are each computed with its own transport equation, the swirling and complex flow field can be resolved to an extent agreeable to the experiment. It can therefore be concluded that it is a valid model for the additional result comparisons discussed below.

\section{(b). $45^{\circ}$ Swirlers Case Study}

The results obtained with the $45^{\circ}$ swirler primarily display the significant differences between swirlers. The magnitude of the inlet velocity is the same as the $60^{\circ}$ swirler case. As Figures 5.4.13 and 5.4.14 indicate, there is no reversed flow at the center of the circles. Since the swirler diverts the upstream air at a smaller angle, a strong and solid forward-flow jet enters the combustor and does not permit recirculation as did the more conical jet from the $60^{\circ}$ swirlers. Due to the lower degree of swirl, both turbulence models produce results almost identical to each other; however, weak recirculation regions 
are captured by the RSTM. Since the $45^{\circ}$ swirler produces such weak swirl flow, these results show that both RSTM and $k-\varepsilon$ models can predict the flow field equally well.

The velocity profiles in Figure 5.4.14 suggests that the atomization, vaporization, and mixing processes would occur less effectively with the $45^{\circ}$ swirler. Fuel and air are injected directly downward into the primary zone, reducing the time for the three processes. Fuel-air jets around the perimeter are directed toward the wall and could lead to fuel droplet impingement on the walls. The lack of recirculation could reduce the amount of shear that aids in fuel atomization, and it could also reduce the degree of mixing that aids in uniform lean combustion.

\section{DISC Engine}

The DISC engine chamber whose $2 \mathrm{D}$ computational domain at $-60^{\circ}$ ATDC is displayed in Figure 5.4.15. The chamber has a bore of $9.843 \mathrm{~cm}$ and a stroke of $9.55 \mathrm{~cm}$. A chamfered bowl is located in the piston, and its cavity depth is $3.3 \mathrm{~cm}$. The piston is located at $-90^{\circ}$ ATDC when the calculation starts. At this time, the fluid inside the cylinder has the composition of pure stagnant air at $400 \mathrm{~K}$ with a Bessel function swirl profile.

During the compression stroke, $11.6 \mathrm{mg}$ of liquid gasoline in the form of a hollow cone spray is injected into the cylinder from an injector with a single half sine wave pulse, located close to the cylinder head axis. The injection begins at $-52^{\circ}$ ATDC and has a duration of $12.672^{\circ}$. Figure 5.4.16 shows the $k-\varepsilon$ and SSG RSTM flow and temperature field results at $-30^{\circ}$ ATDC, shortly before ignition.

The fuel-air mixture is subsequently spark-ignited at $27^{\circ}$ ATDC. Drastic differences between RSTM and $k-\varepsilon$ model results can be observed from the figures. The magnitude of the eddy viscosity is overpredicted by the ? e model, which is expected since 
the model typically overpredicts t. This can be seen in Figure 5.4.17 for the effective viscosity. Because of the smaller eddy viscosity, the SSG RSTM results tend to show more distinct and localized profiles of temperature and effective viscosity. For example, the cooler region corresponding to the fuel spray at $-30^{\circ}$ ATDC is smaller and less diffuse for the RSTM model. In addition, the RSTM is able to perceive the spray in the effective viscosity contour plot. Distinctive profiles also exist at later angles as in Figure 5.4.18, which focuses only on the bowl region. Here the high temperature combustion gases are confined near the center of the bowl while several squish-induced circulation regions dominate the RSTM velocity profile. Highly diffusive variations are seen from the $k$ - $\varepsilon$ results while only a defined structure appears from the RSTM. As shown, the anisotropic nature of the flowfield is resolved only by the RSTM.

\section{$\underline{\text { LPP Combustor }}$}

The SSG RSTM was also applied to propane-air combustion in a lean, premixed, prevaporized (LPP) flametube [Anderson, 1975]. A study has been recently performed by Kundu et al. [1998] with the $k-\varepsilon$ model and with a reduced reaction mechanism (23 kinetic reactions with 16 species) to simulate the combustion and to evaluate NOx emissions. The computational region displayed in Figure 5.4 .19 is $43 \mathrm{~cm}$ long and $10.2 \mathrm{~cm}$ in diameter. It includes the combustion zone, the flame holder, and a $10 \mathrm{~cm}$ length of the prevaporization/ premixing zone. To reduce the domain into an axisymmetric model, the holes of the actual flame holder were approximated by several concentric rings. Therefore, only 4434 cells were required for a 2D radial mesh. An inlet velocity of $2500 \mathrm{~cm} / \mathrm{s}$ and temperature of $800 \mathrm{~K}$ were specified in addition to an equivalence ratio of 0.8 . 
The results show differences between the RSTM and $k-\varepsilon$ models in terms of the velocity profile and $\mathrm{NO}_{\mathrm{x}}$ emission index. Figure 5.4.20 illustrates the computed velocity vectors for both models. It is apparent that the solution with the SSG model produces a large circulation zone on the wall-side of the flame tube that is not produced by the $k-\varepsilon$ model. In fact, the $k-\varepsilon$ model produces a velocity field that does not show any significant variation over the diameter of the flame tube, irrespective of the varying slot area and the much larger slot adjacent to the wall. The RSTM produces a velocity field that appears realistically descriptive of the fluid dynamics. This cannot be explicitly verified as there is no detailed experimental data of the velocity field for this particular case.

There is, however, experimental data of NO concentrations for this csae. This data is given in the contour plots at the bottom of Figure 5.4.21 and represents the measured EI (g of Nox $/ \mathrm{kg}$ of fuel) along the centerline of the flame tube. In general, the levels of NO produced with the RSTM are closer to experimental values than those with the twoequation model.

\section{4-Valve DOHC Engine}

The last example shows the calculation of a 4-valve DOHC engine using the standard $k$ - $\varepsilon$ model in the KIVA code and the LRR RSTM without the wall-reflection term. The engine under consideration is 1200 c.c. with four cylinders and group port injection. Each cylinder has two intake and two exhaust valves and are canted at 100 . The engine has a compression ratio of 9.5, a $7.45 \mathrm{~cm}$ cylinder diameter (bore); $6.87 \mathrm{~cm}$ stroke, $11.27 \mathrm{~cm}$ connecting rod length, and operates at 1,500 rpm. Figure 5.4.22 shows the geometry and computational mesh of the DOHC engine. The valve lift history is given in Figure 5.4.23. 
Figure 5.4.24 shows the velocity vectors at different crank angles during intake and exhaust strokes. At the beginning of the intake, high-velocity air is drawn in through the intake valve and produces vortices below it, Figure 5.4.24a. Both models show similar flow patterns at this crank angle $(\mathrm{CA})$. At $\mathrm{CA}=1200$, just past the maximum lift (see Figure 23), visible flow differences can be seen in Fig. 24b. Two vortices, one underneath the intake valve and the other near the bottom of the piston surface, are clearly shown in the LRR model prediction. Toward the end of the intake process, at CA $=1800$ (Figure 24c), the vortex structures predicted by these two models are quite different, although the general flow patterns are somewhat similar. Hascher et al. [Hascher et al., 2000] have also made similar observation when the experimental results were compared with the $k-\varepsilon$ model predictions using the Star-CD code. To gain a better understanding of the flow structure at this particular crank angle, Figure 5.4.25 shows the three-dimensional color-coded velocity vectors for both models. It can be seen that, due to the canted valves and the piston motion, the LRR model predicts stronger swirl flow. Near the peak of the exhaust stroke at $\mathrm{CA}=6000$, Figure $5.4 .24 \mathrm{~d}$, both models predict similar flow pattern, since relatively low level of turbulence exists in the flow field.

\subsubsection{Conclusion}

Two RSTMs were implemented within the KIVA code. Five application cases were studied to ascertain the performance of the RSTM by comparing the results with the $k$ - $\varepsilon$ model predictions and the LDV measurements. Incorporated into the structure of the KIVA code, the RSTM was shown to successfully reproduce the mean flow features and second moments for a range of standard turbulent flow test cases, which include planar 
and axisymmetric flows5. This provides confidence in applying the RSTM to engine type configurations. In these cases, the RSTM produces velocity and eddy viscosity fields that differ from the $k-\varepsilon$ model. Comparisons with the available, though limited, experiments, show that the RSTM results are more realistic. Unlike the $k$ - $\varepsilon$ model, the RSTM is able to capture most recirculation structures, including those arising from the turbulent-stress anisotropy. In the studies of two LDI combustor cases, it further reveals that, for high degree swirl flows, the RSTM outperforms the $k-\varepsilon$ model. Further validation of the RSTM will be obtained in the future when compared with additional experimental measurements. 

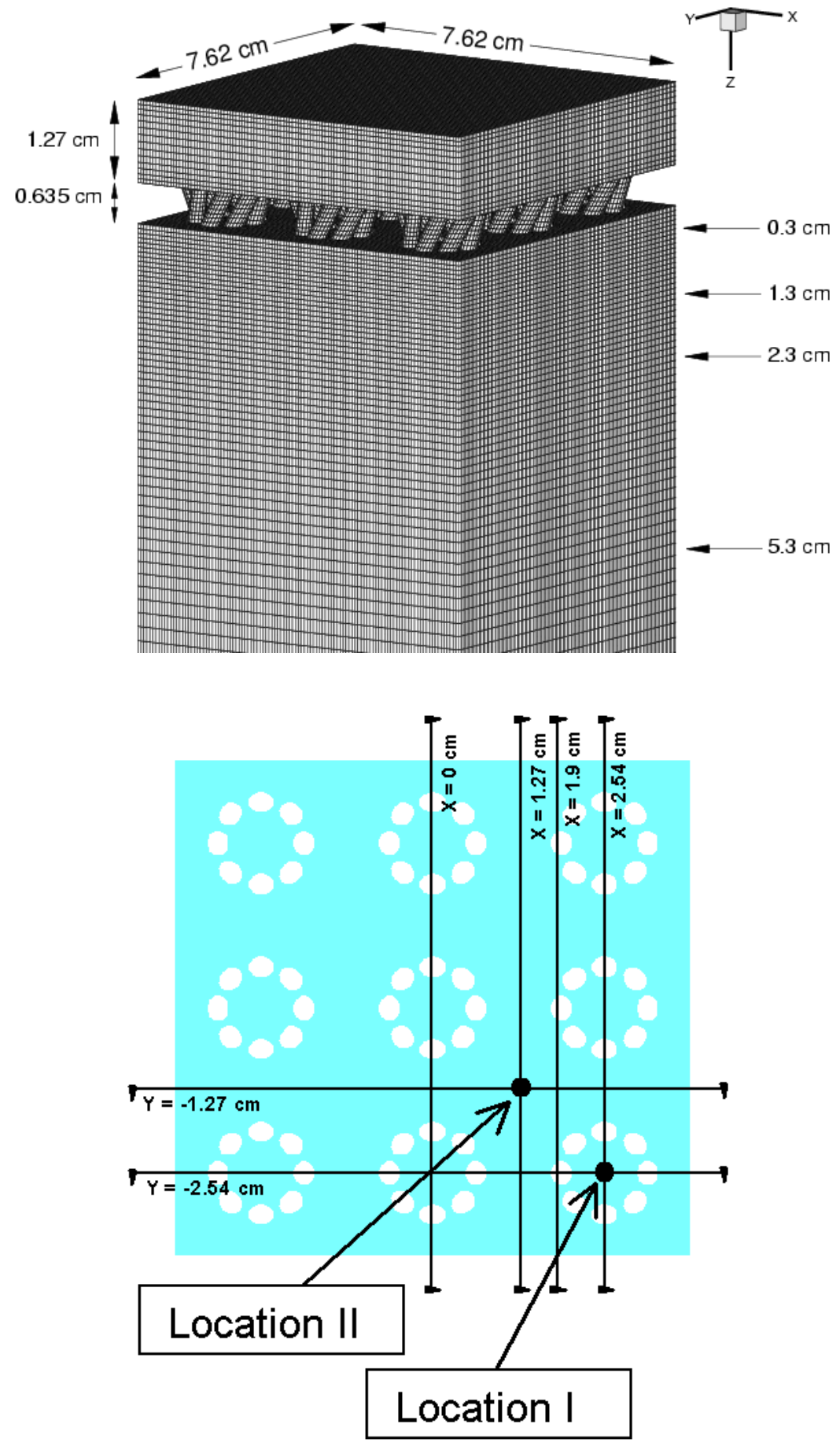

Figure 5.4.1. Computational Grid of the Entier Domain, Dimensions, and Cutting Planes 


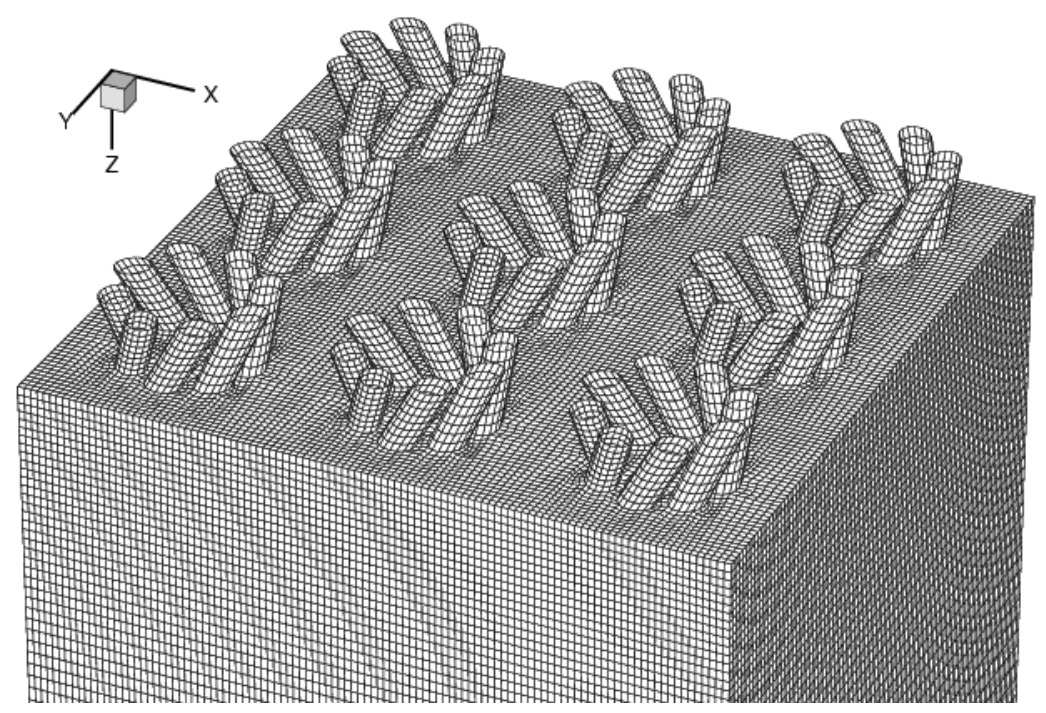

Figure 5.4.2. Discrete-Jet Swirlers in 3x3 Pattern Atop a Burner Duct

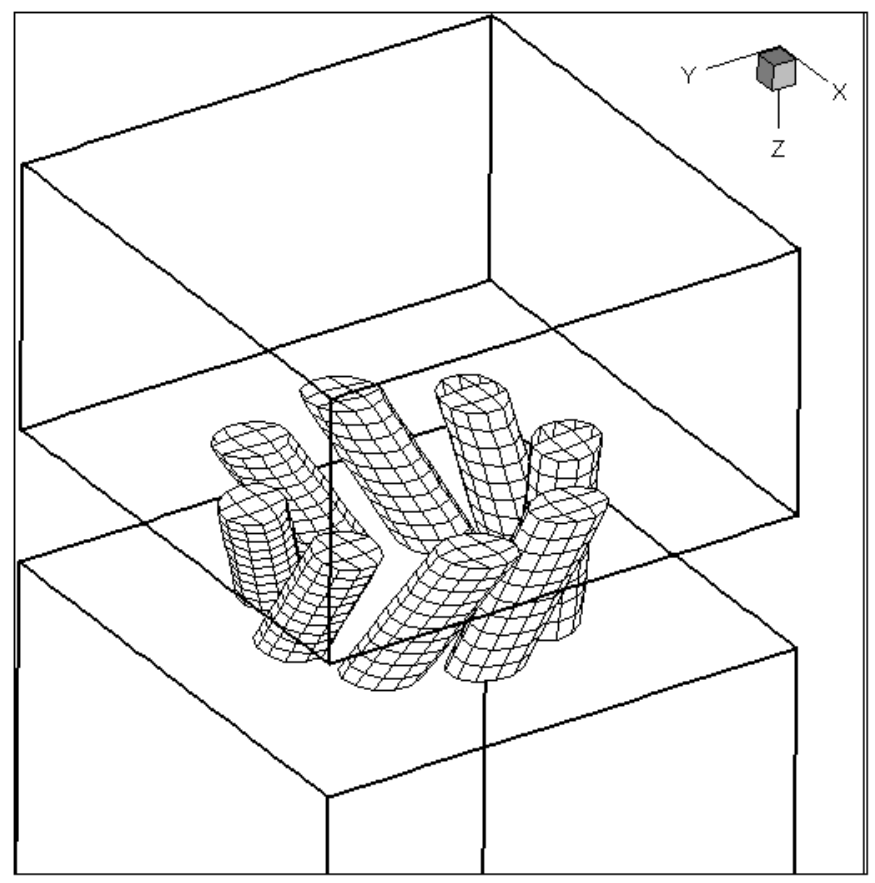

Figure 5.4.3. Computational Grid of a Single Discrete-Jet Swirler 


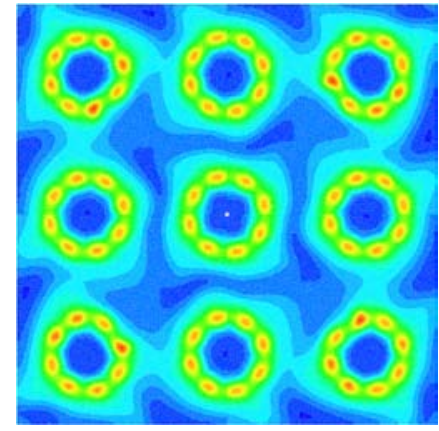

(a) NCC $k-\varepsilon$

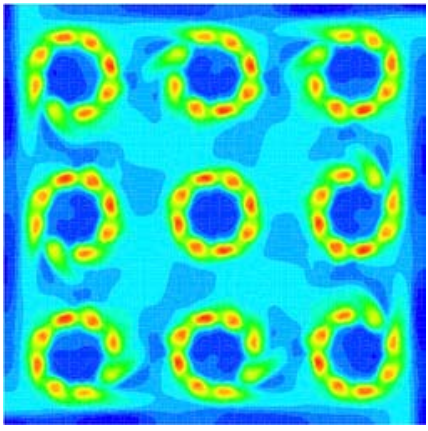

(b) RSTM

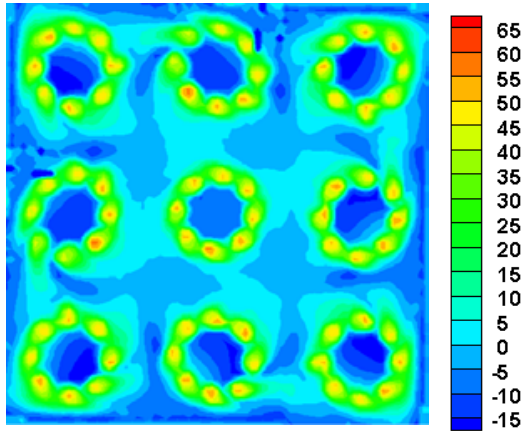

(c) LDV Measurements

Figure 5.4.4. Comparison of Axial Velocity at $Z=3 \mathrm{~mm}$

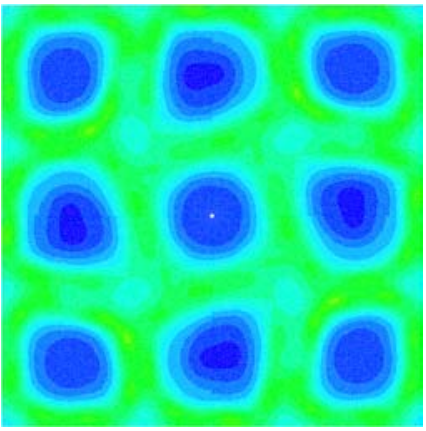

(a) NCC $k-\varepsilon$

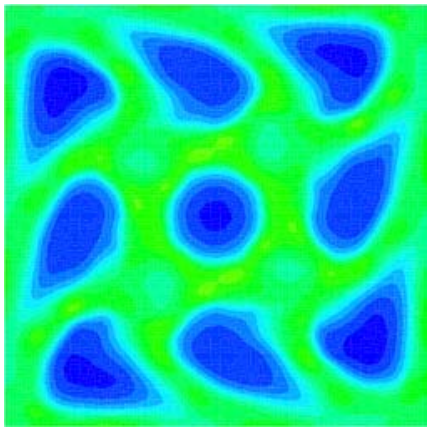

(b) RSTM

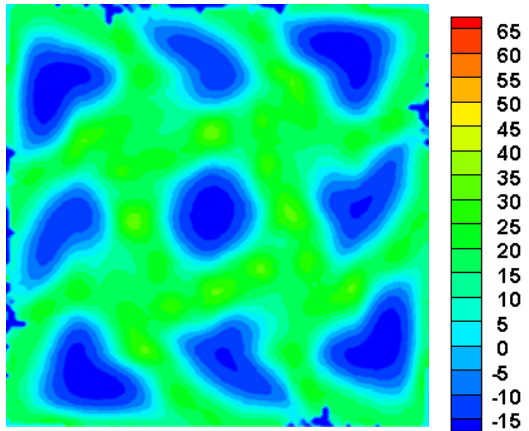

(c) LDV Measurements

Figure 5.4.5. Comparison of Axial Velocity at $Z=13 \mathrm{~mm}$ 


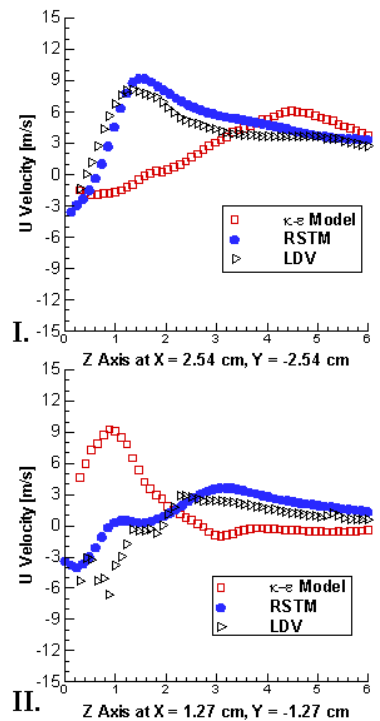

(a) U velocity

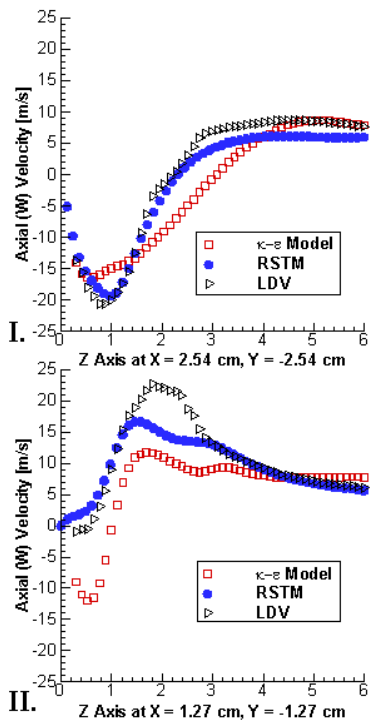

(b) $\mathrm{W}$ (axial) velocity

Figure 5.4.6. Line Plots of Velocity Components

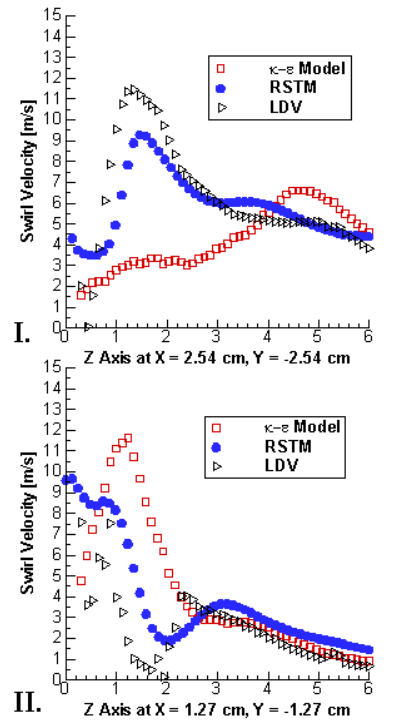

(a) Swirl velocity

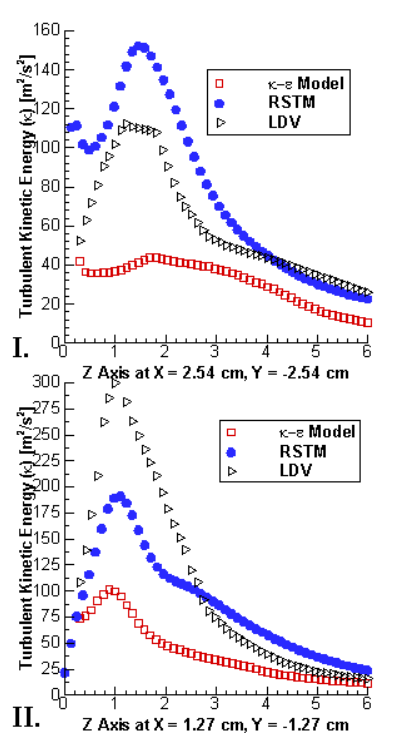

(b) Turbulent Kinetic Energy

Figure 5.4.7. Line Plots of Swirl Velocity and Turbulent Kinetic Energy 


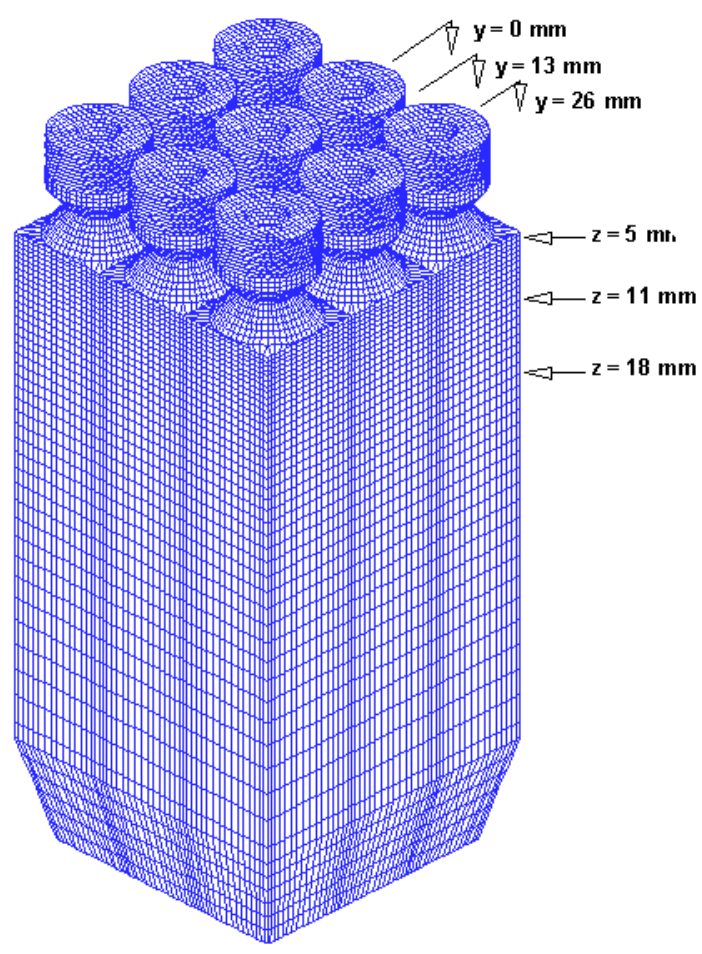

Figure 5.4.8. Computational Model and Cutting Planes

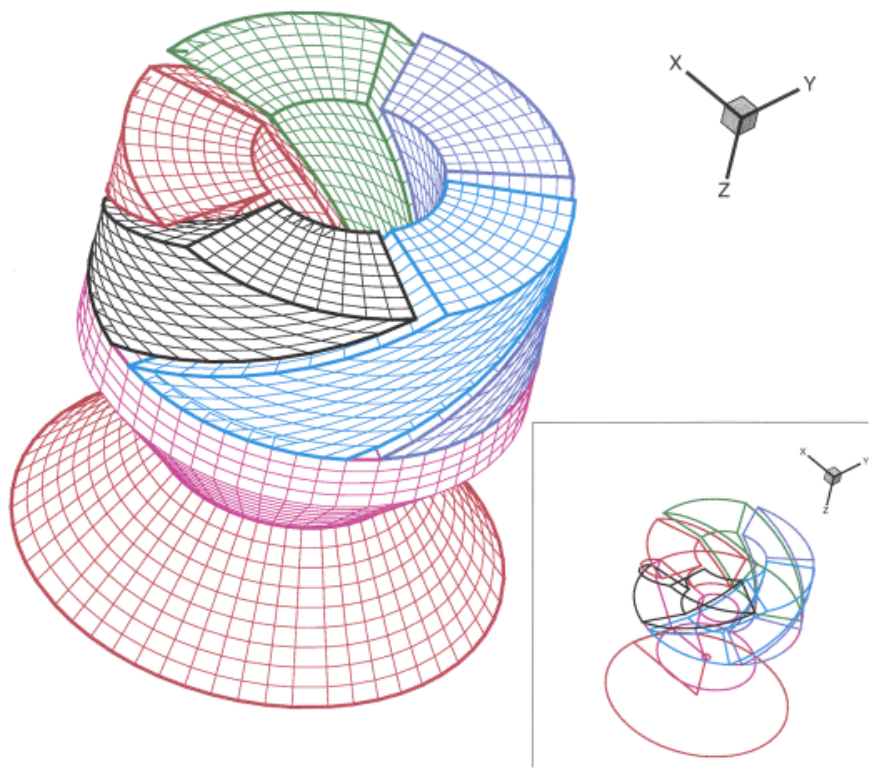

Figure 5.4.9. A Sketch of the Axial Swirler 

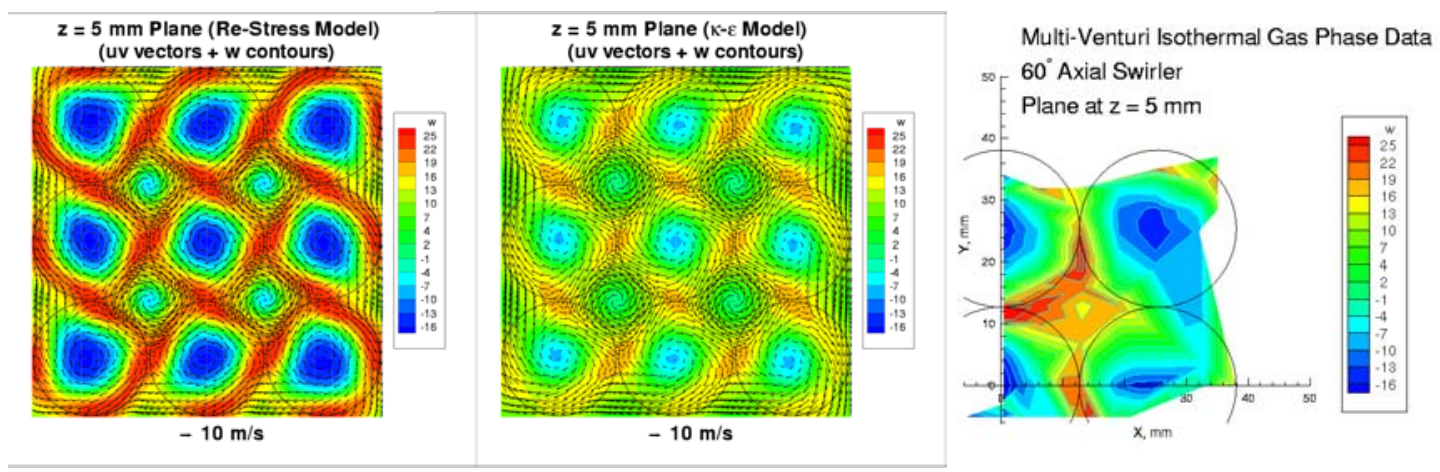

Figure 5.4.10. Results for $60^{\circ}$ Swirler at $Z=5 \mathrm{~mm}$ Cutting Plane
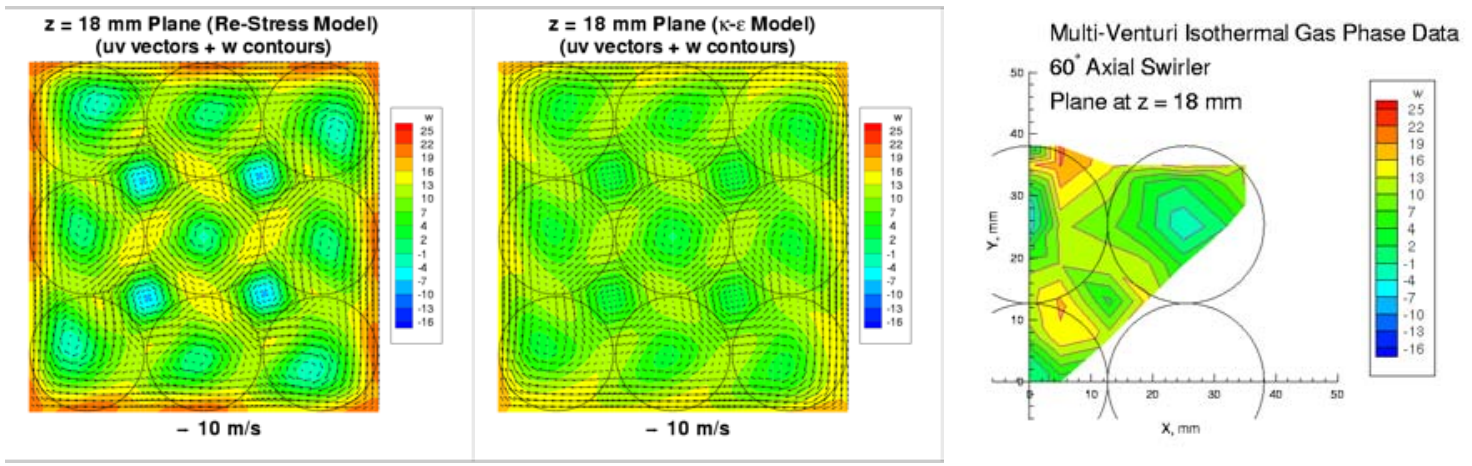

Figure 5.4.11. Results for $60^{\circ}$ Swirler at $Z=18 \mathrm{~mm}$ Cutting Plane

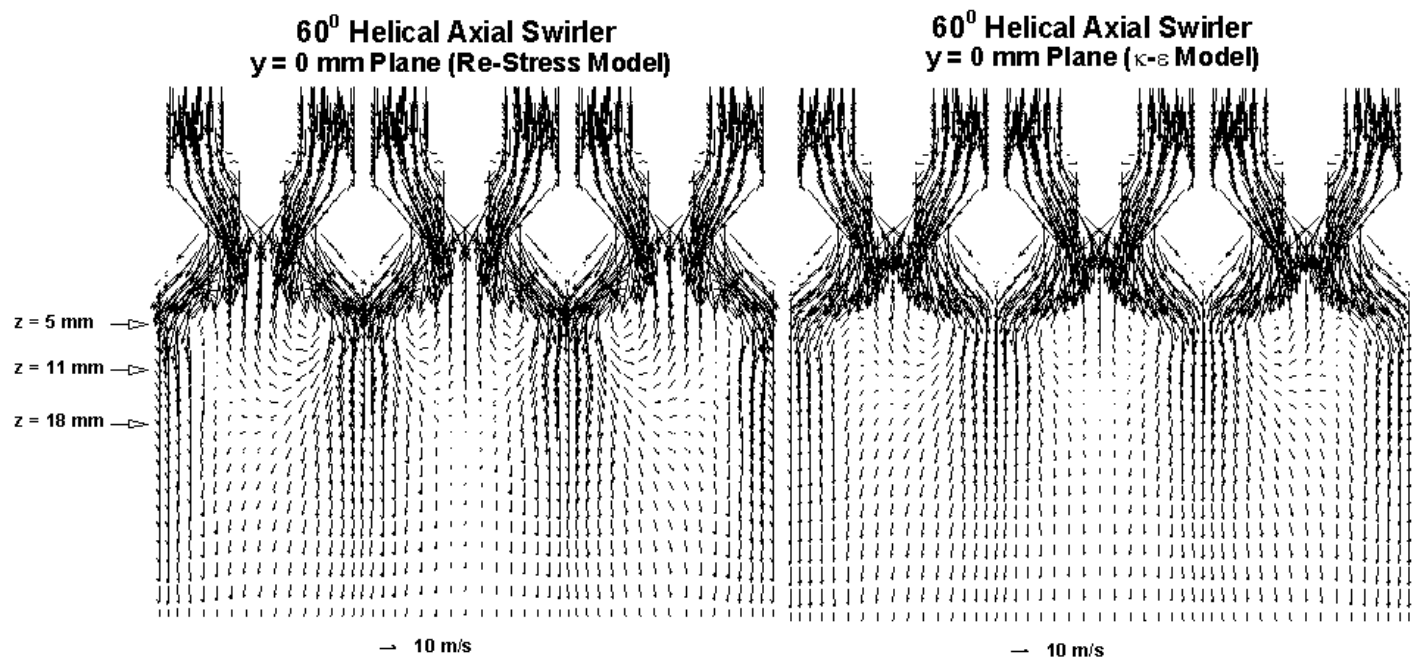

Figure 5.4.12. Velocity Vectors on $\mathrm{Y}=0.0 \mathrm{~mm}$ Cutting Plane for $60^{\circ}$ Swirler 

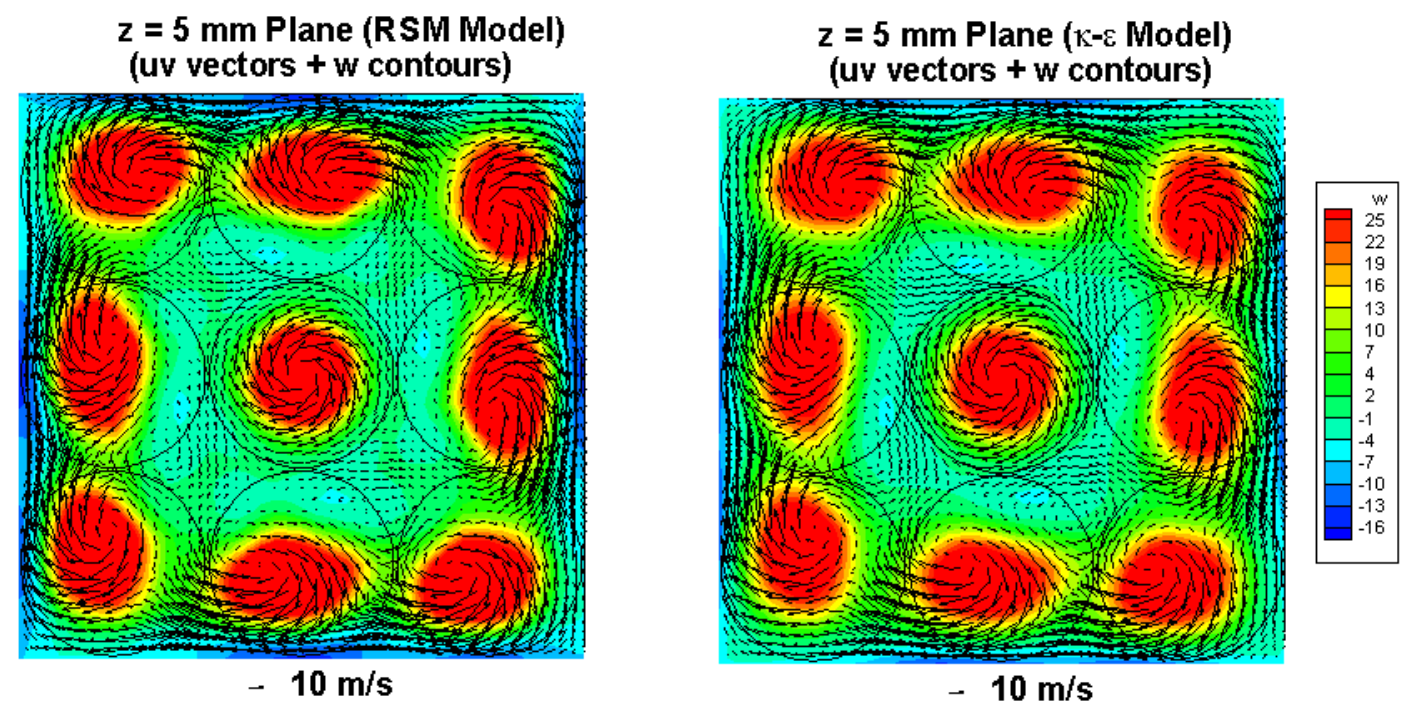

Figure 5.4.13. Results for $45^{\circ}$ Swirler at $Z=5 \mathrm{~mm}$ Cutting Plane

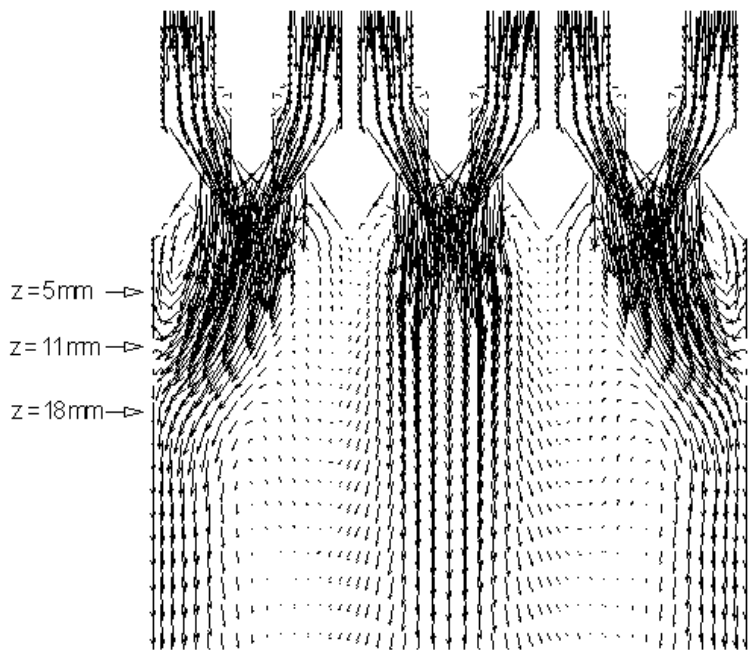

- $10 \mathrm{~m}$ 's

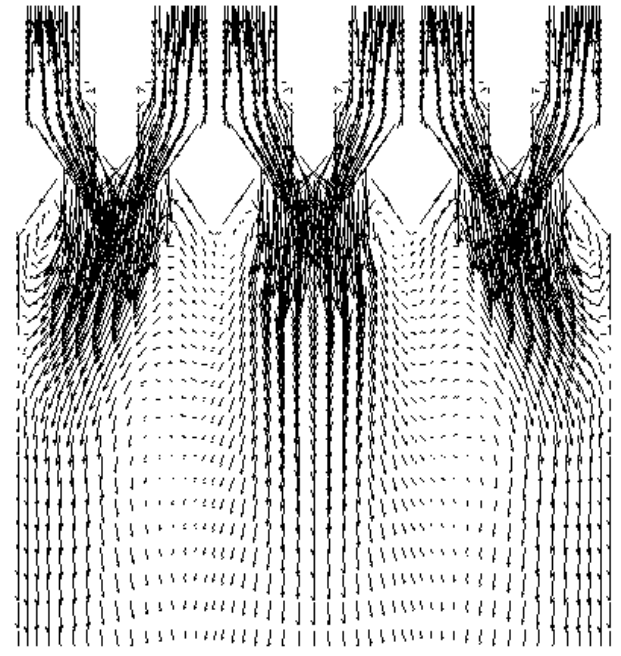

$\rightarrow 10 \mathrm{~m} / \mathrm{s}$

Figure 5.4.14. Velocity Vectors on $\mathrm{Y}=0.0 \mathrm{~mm}$ Cutting Plane for $45^{\circ}$ Swirler 


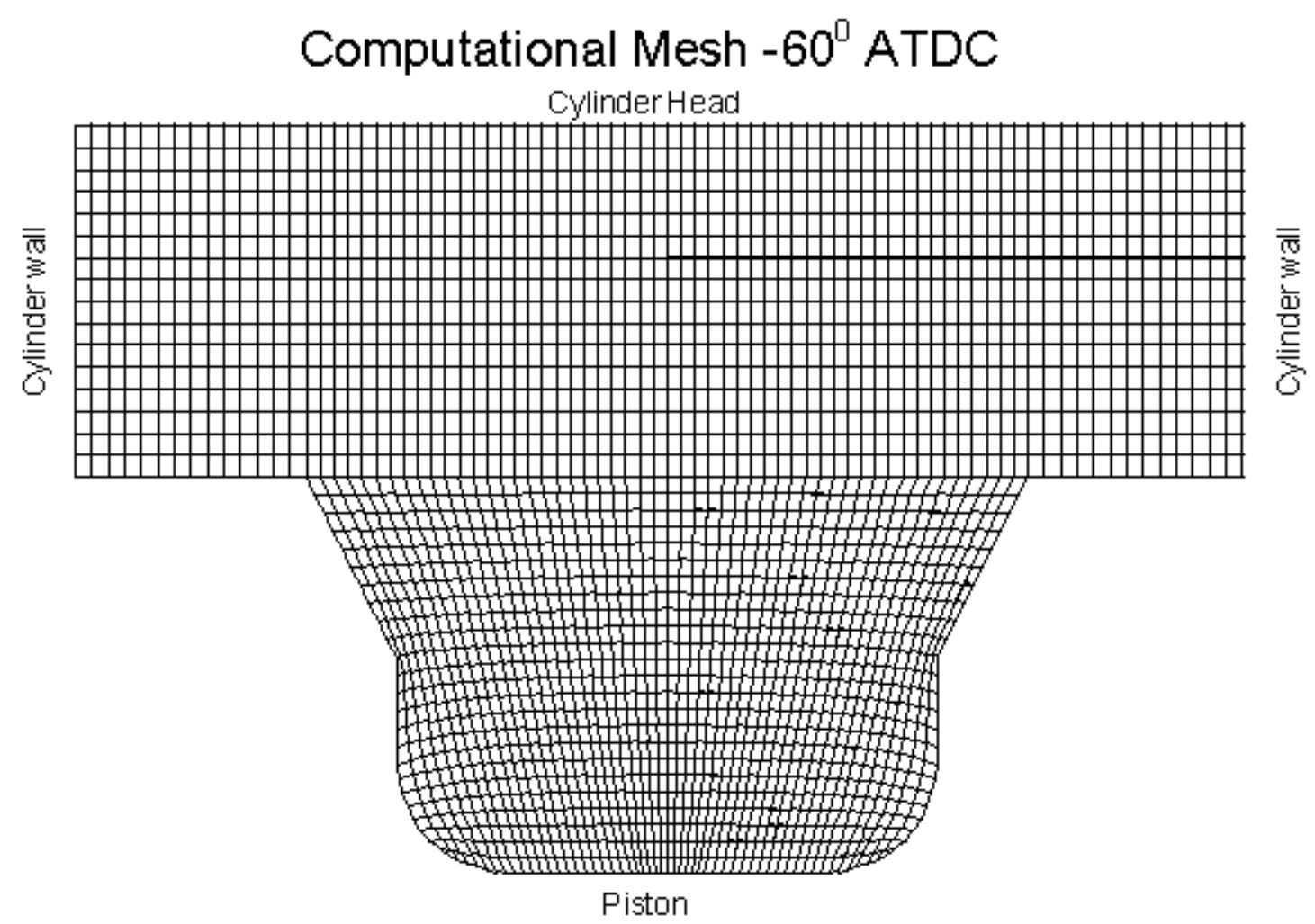

Figure 5.4.15. DISC Computational Mesh

\section{Velocity and Temperature at $-30^{\circ}$ ATDC}

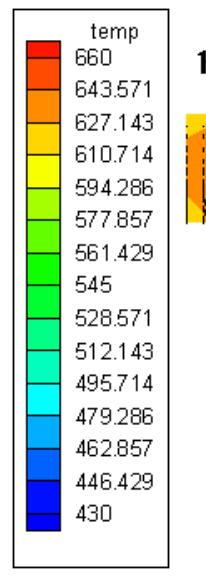

\section{א- $\varepsilon$ Model Re-Stress Model}

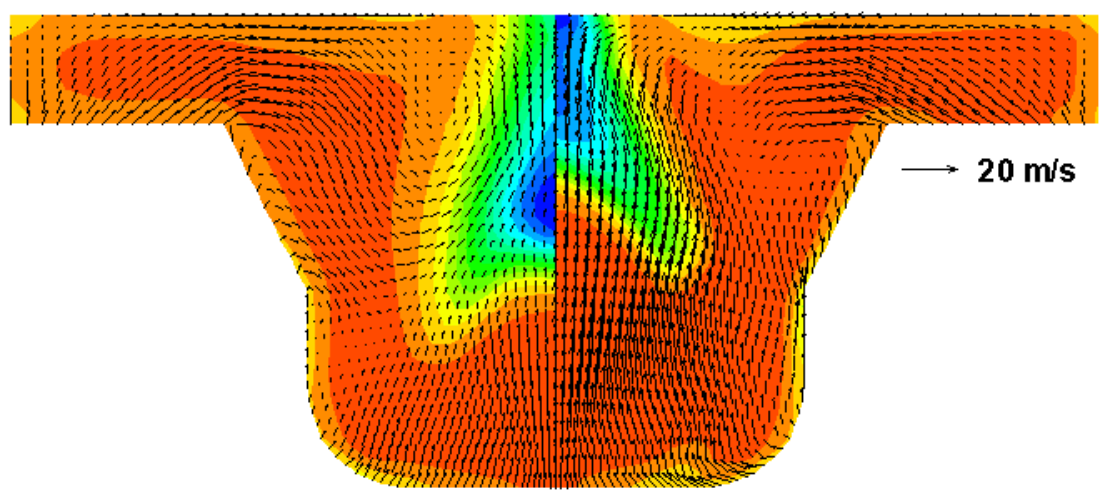

Figure 5.4.16. Velocity Vector and Temperature at $-30^{\circ}$ ATDC 


\section{Effective Viscosity $\mu_{\text {eff }}$ Contours at $-30^{\circ}$ ATDC}

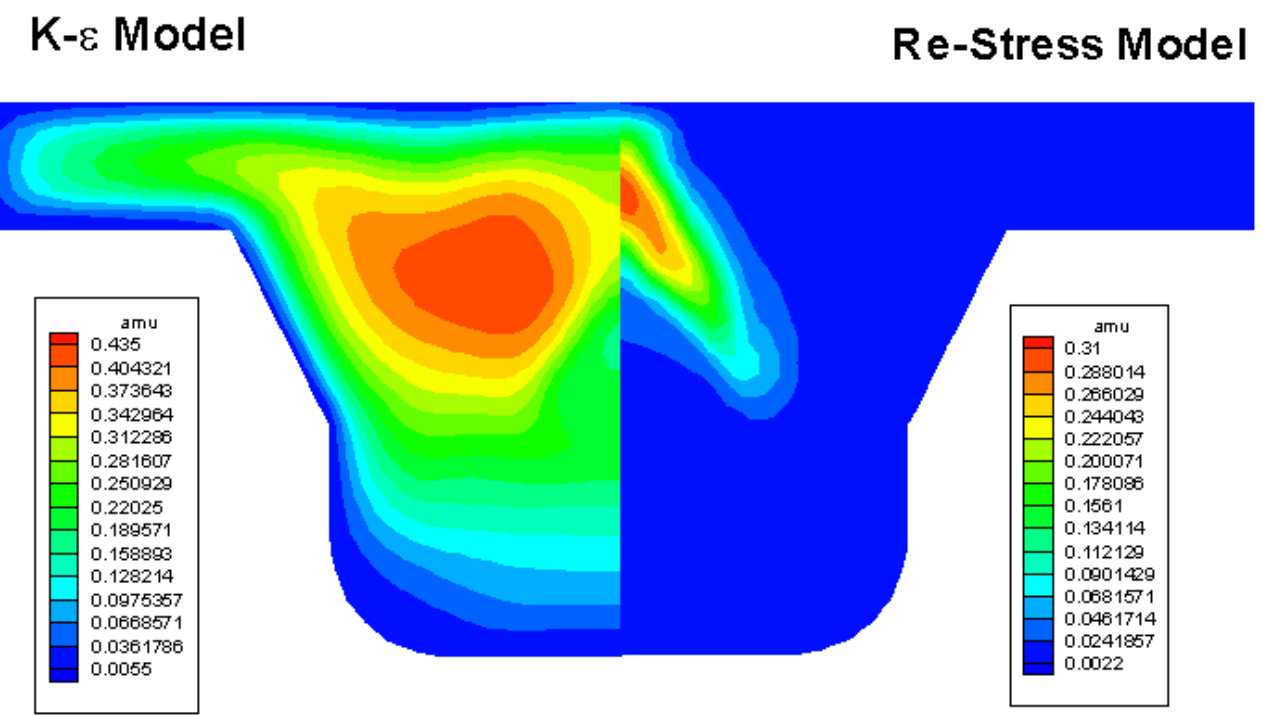

Figure 5.4.17. Effective Viscosity at $-30^{\circ}$ ATDC

\section{Velocity and Temperature at $0^{\circ}$ ATDC}

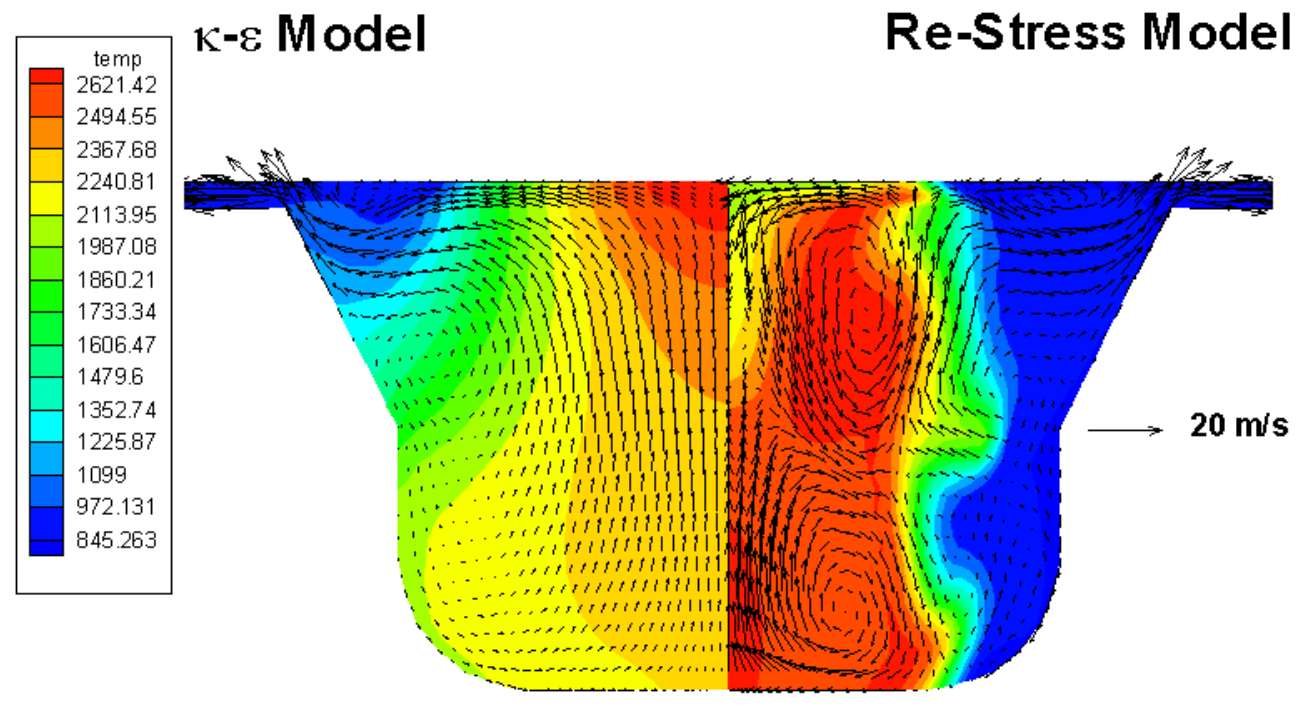

Figure 5.4.18. Velocity Vector and Temperature at $0^{\circ}$ ATDC 


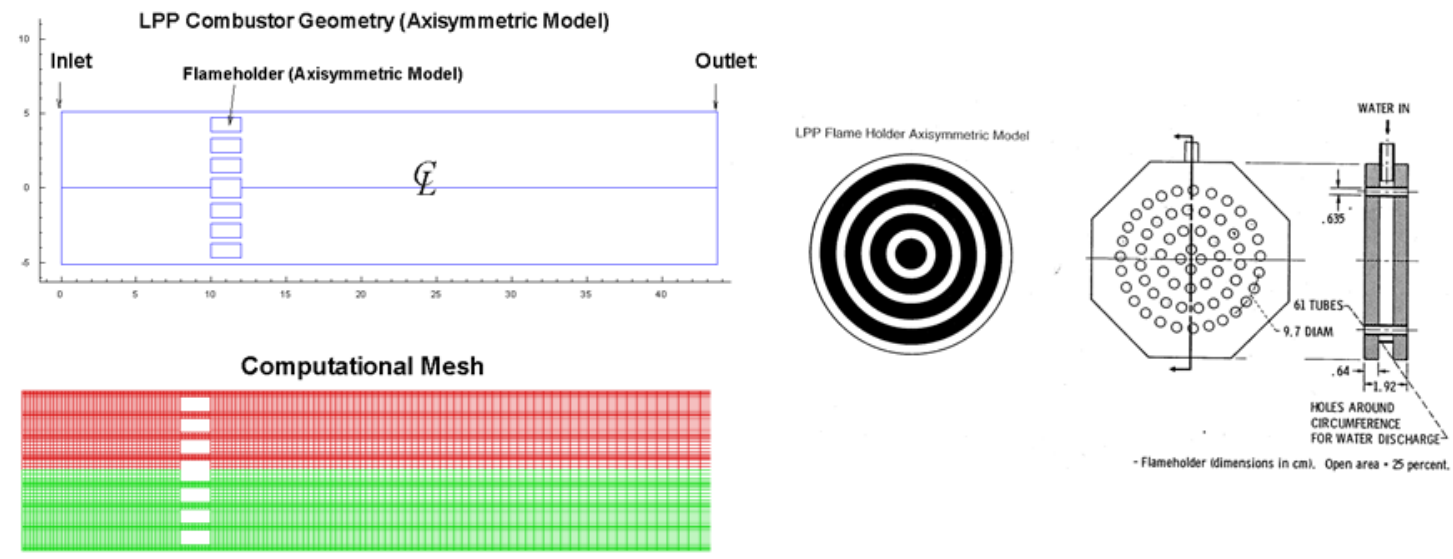

Figure 5.4.19. LPP Combustor Computational Model, Mesh, and Flame-Holder

$\longrightarrow 300 \mathrm{~m} / \mathrm{s}$
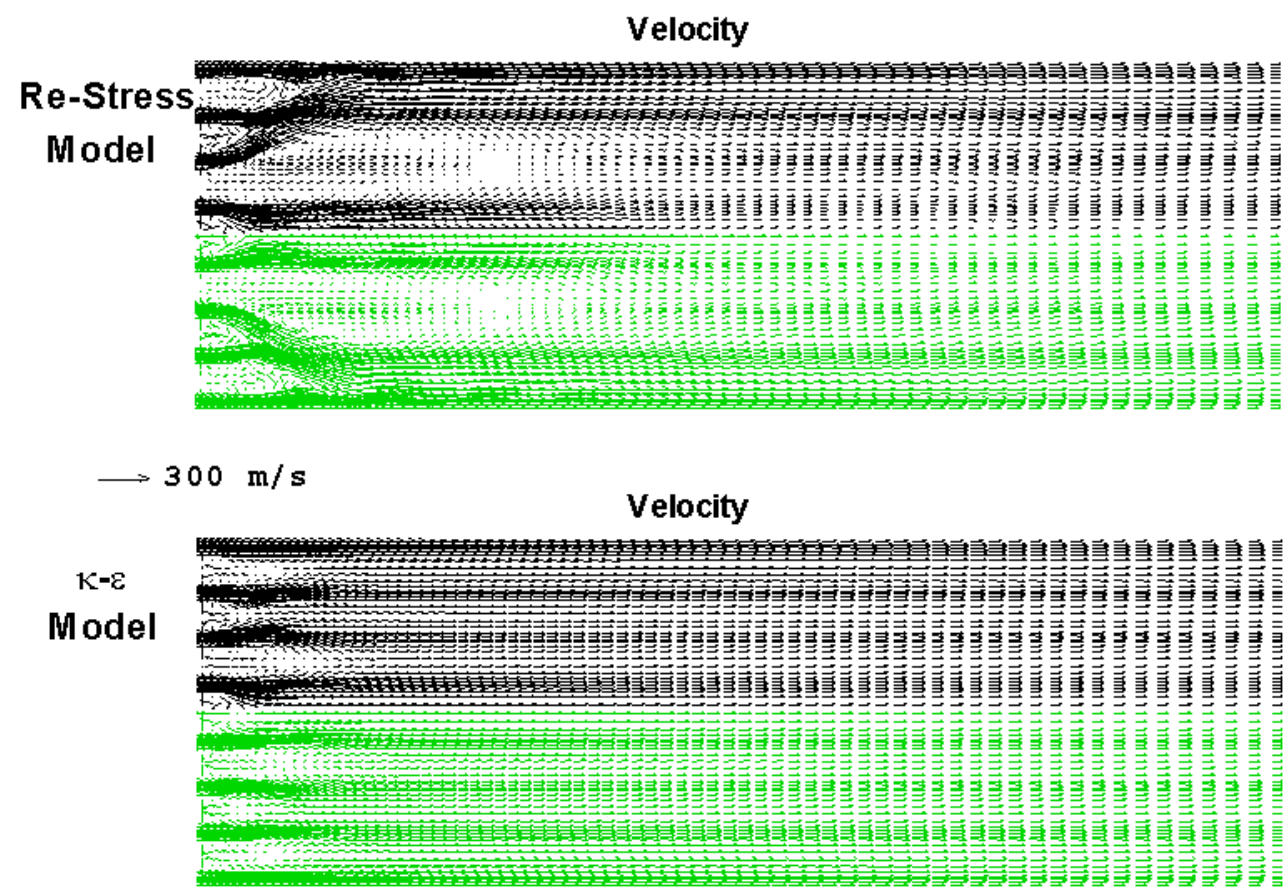

Figure 5.4.20. Velocity Vector 

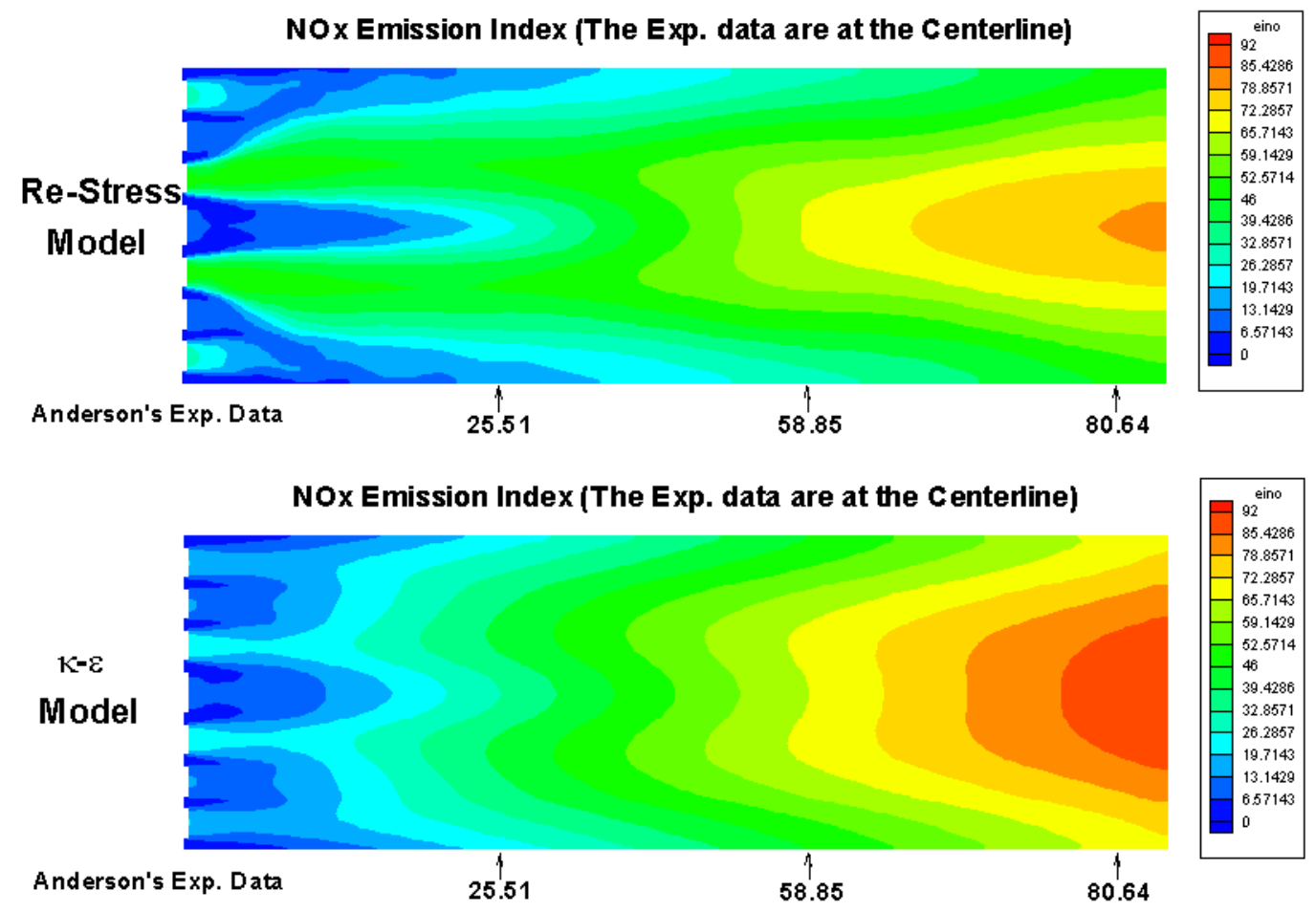

Figure 5.4. 21. $\mathrm{NO}_{\mathrm{x}}$ Emission Index

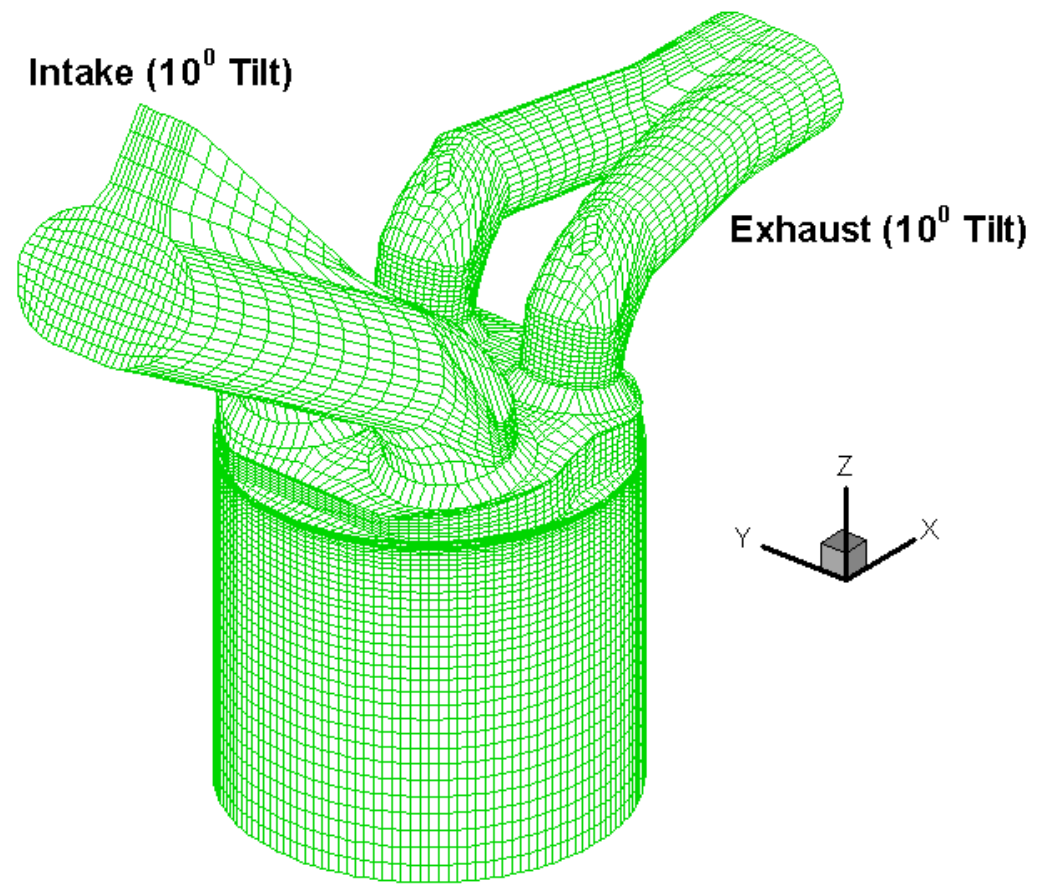

Figure 5.4.22. 4-Valve DOHC Computational Mesh 


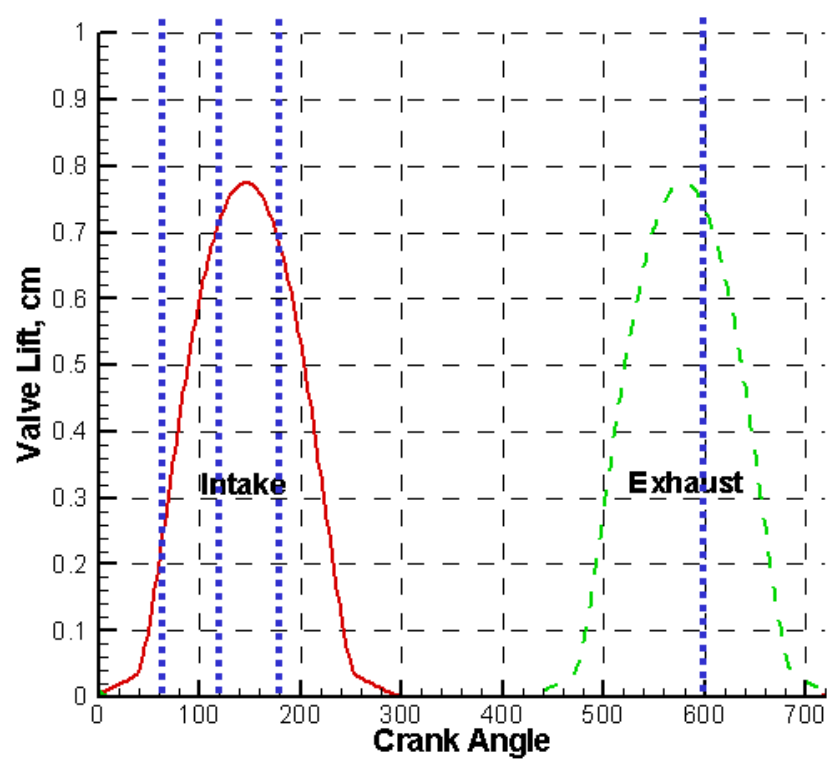

Figure 5.4.23. Valve Lift History

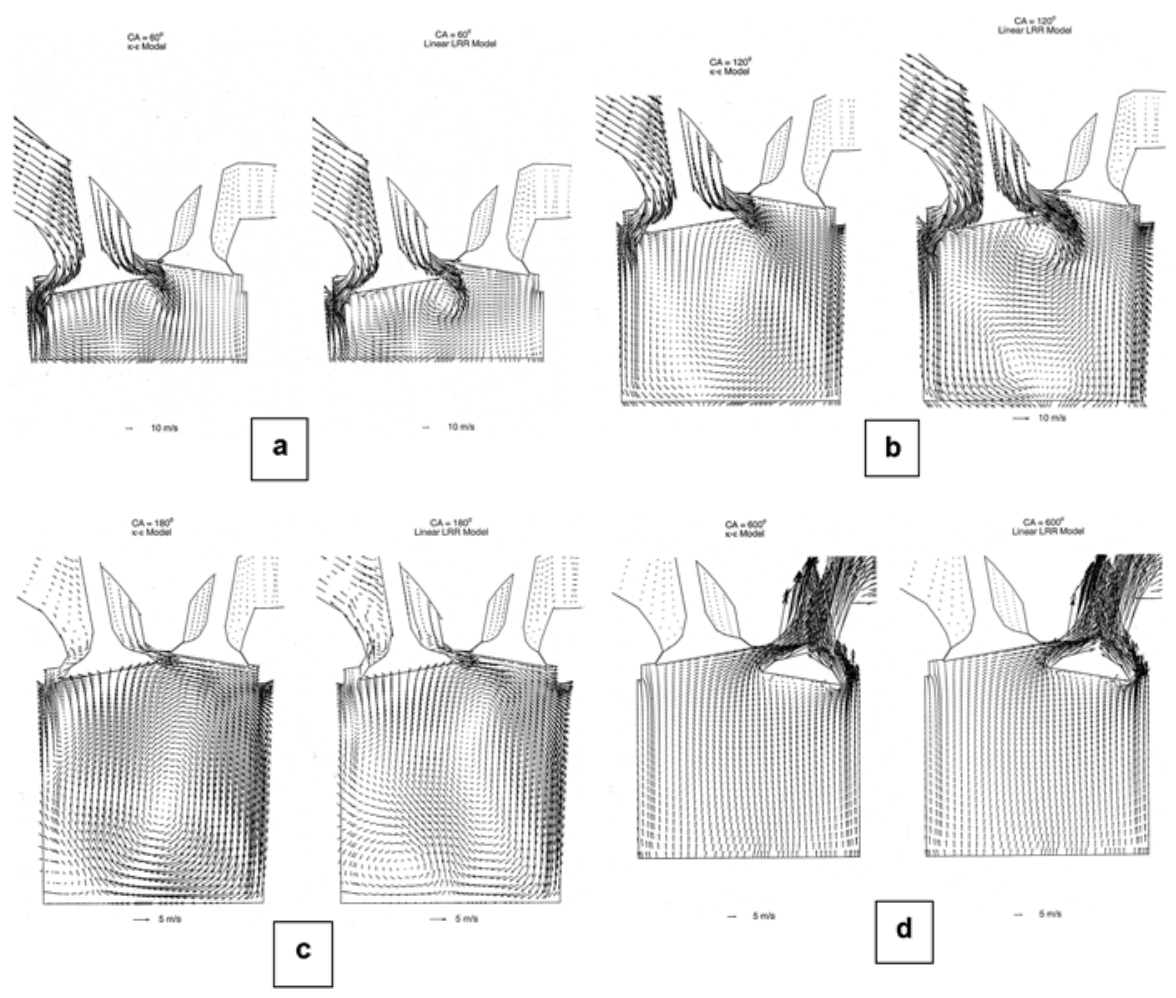

Figure 5.4.24. Velocity Vector at $C A=60^{\circ}, 120^{\circ}, 180^{\circ}$, and $600^{\circ}$ 


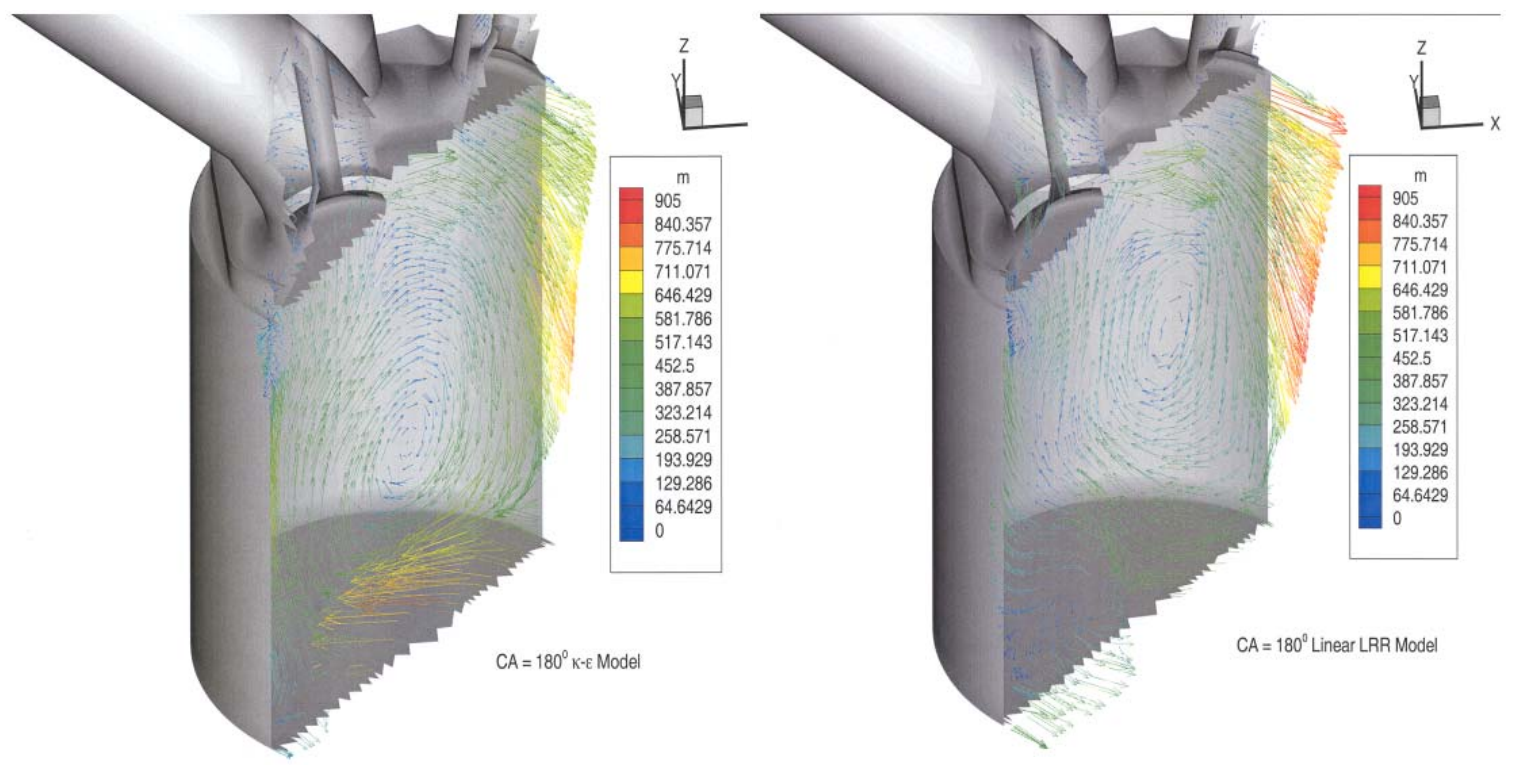

Figure 5.4.25. 3-D Velocity Vector at $\mathrm{CA}=180^{\circ}$ 


\subsection{LDI Combustor with Radial Swirlers}

This study is an extension of the application of RSTM in combustor design described in Section 5.1. The primary differences are the design and total number of the swirlers. Here, sixteen swirlers with radial inlet (instead of axial) are used. These swirlers are arranged in a four-by-four pattern on top of a lean-direct-injection (LDI) combustor, as shown in Figure 5.5.1. The combustion chamber has the same geometry, dimension, and orientation as in the previous section. The sixteen swirlers, also equally spaced, therefore, now has smaller diameter compared to the axial-swirler case.

Each swirler has a central hollow slot to allow for the fuel injector (Figure 5.5.2). The current study, however, is a cold-flow analysis of the fluid flow field. Therefore, these hollow space is merely a place-holder. Compressed air enters the swirlers through openings in the radial direction as Figure 5.5.3 indicates. Each inlet opening has an imaginary plane that guides the airflow at a vane angle of $60^{\circ}$ as Figure 5.5.4 shows, with the aid of velocity vectors. All sixteen swirlers have the same vane angle in the same counter-clockwise (ccw) direction as viewed upstream from the swirlers.

The operating condition is similar to that in the 3-by-3 axial-swirler study.

\subsubsection{Results and Discussion}

No experimental data is available at the present time. However, qualitative evaluation of Reynolds-stress turbulence model can nevertheless be accomplished, by comparing simulation results from the Launder-Reece-Rodi (LRR) RSTM, standard $k-\varepsilon$

model, and renormalization-group (RNG) $k$ - $\varepsilon$ model. Results are presented for various cutting planes - cross-flow and axial, showing velocity vectors and magnitude. The three 
cutting planes normal to the axial $(z)$ direction, namely $z=5 \mathrm{~mm}, 11 \mathrm{~mm}$, and $18 \mathrm{~mm}$ downstream of the radial swirlers, are indicated in Figure 5.5.1. In addition, velocity data from four cutting planes along the streamwise $(z-)$ direction are to be presented subsequently.

Figures 5.5.5 through 5.5.7 show, in three constant-z (or $x y$-) cutting planes, the axial velocity contours superimposed with $u v$-velocity vectors. In general, the standard and RNG $k$ - $\varepsilon$ models predicted similar profiles, including the magnitude of $u v$-velocity and the size and location of the forward and reversed flow $( \pm z)$ regions. However, both $k-\varepsilon$ models computed values noticeably lower when compared with the Reynolds-stress result. Differences in the forward axial velocity, areas flooded in red, and reversed velocity, blue, are especially pronounced at $z=11 \mathrm{~mm}$ and $18 \mathrm{~mm}$. Such distinction is consistent with the 3-by-3 axial swirler study presented previously, and clearly shows the diffusive nature of the two-equation turbulence models.

Figures 5.5.8 through 5.5.11 present visualization of the fluid structure via colorcoded velocity vectors along four streamwise cutting planes. These planes slice through either between two rows of swirlers or the mid-plane of one row of swirlers themselves. Other than comparing the velocity magnitude, these plots also effectively illustrate the depth of the recirculation bubbles downstream of each swirler. 


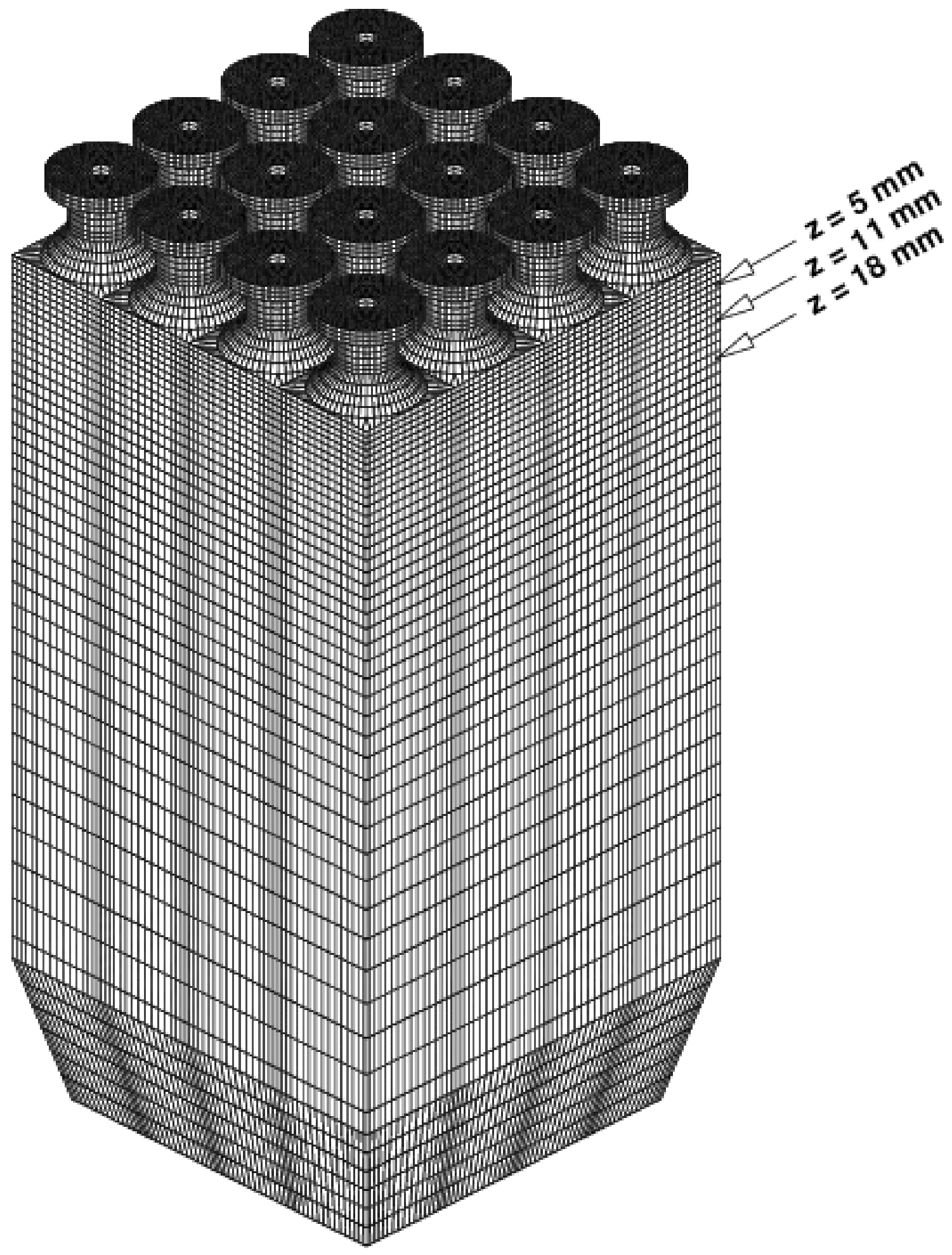

Figure 5.5.1. Computational Mesh of LDI Combustor with Radial Swirlers 


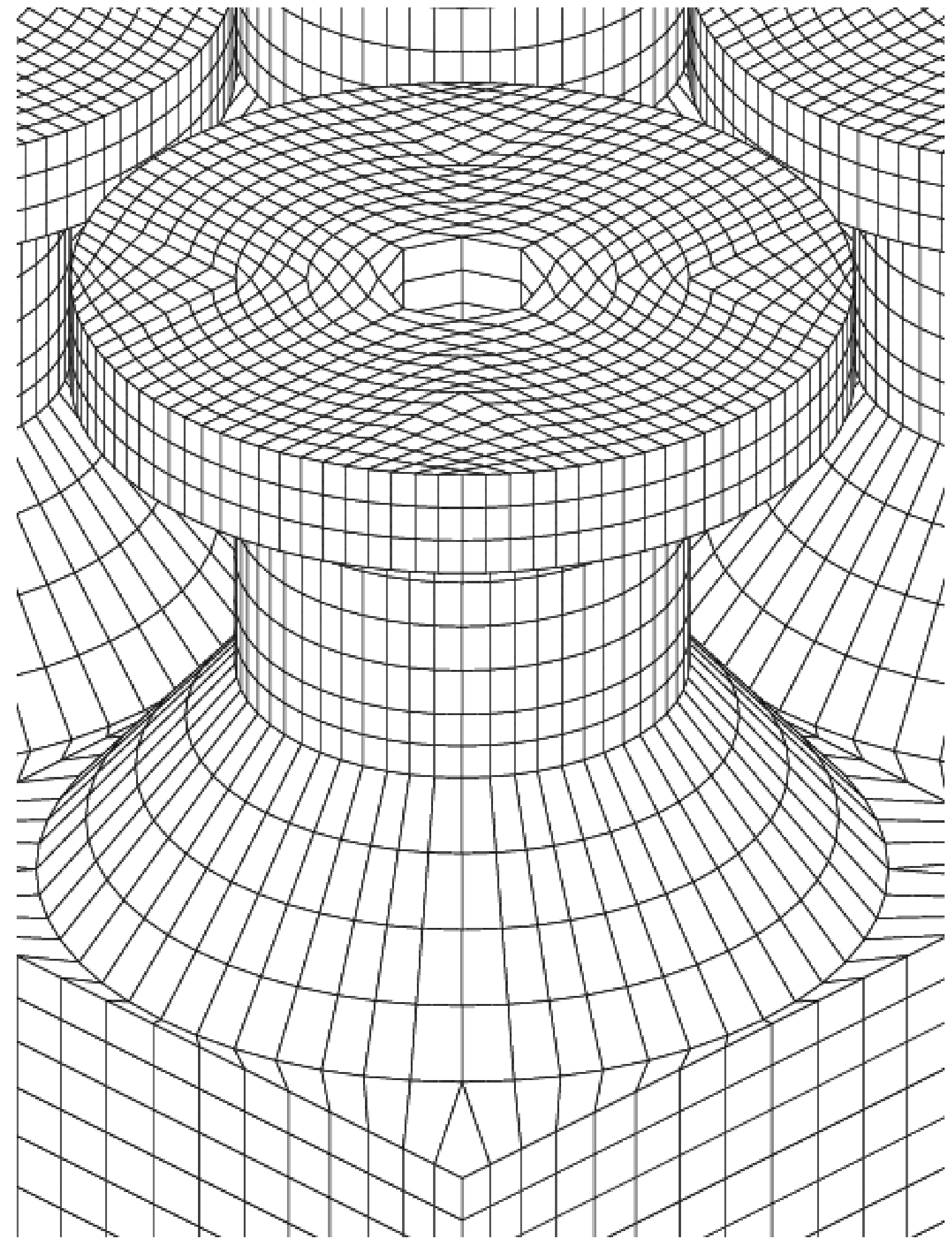

Figure 5.5.2. Close-up View of a Radial Swirler 


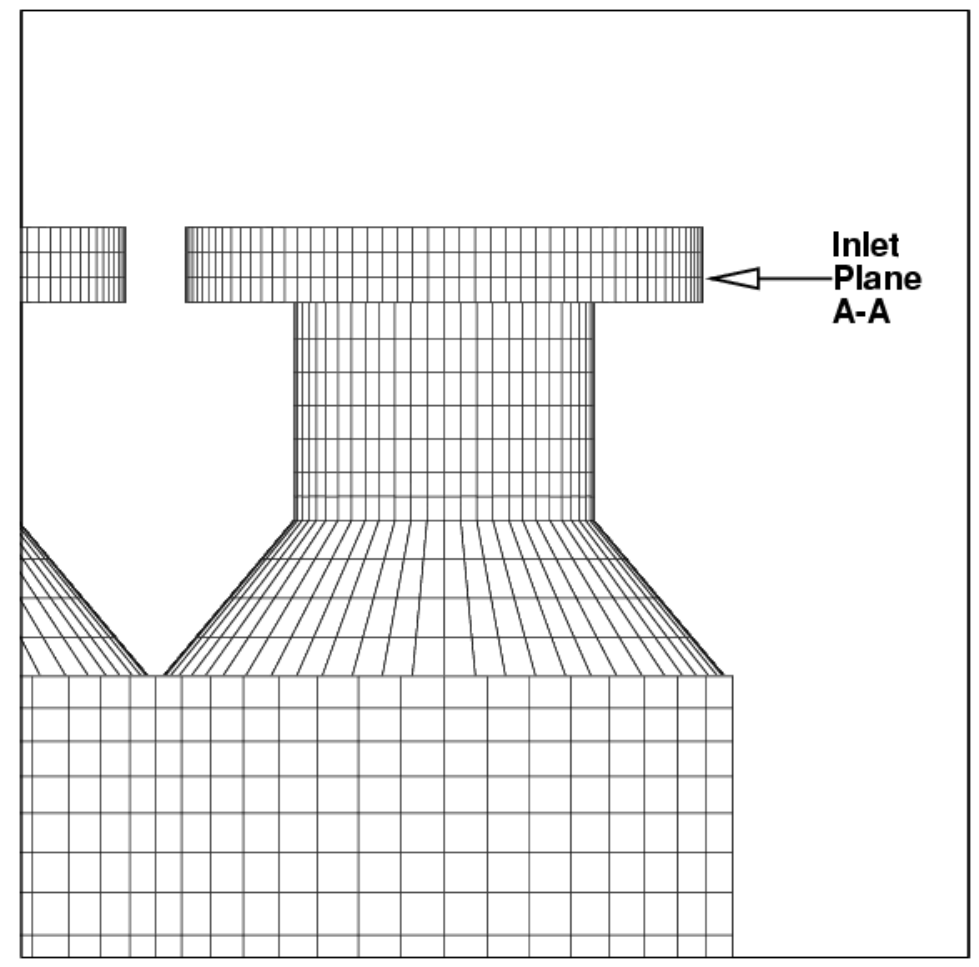

Figure 5.5.3. Orientation of Inlet Air Flow

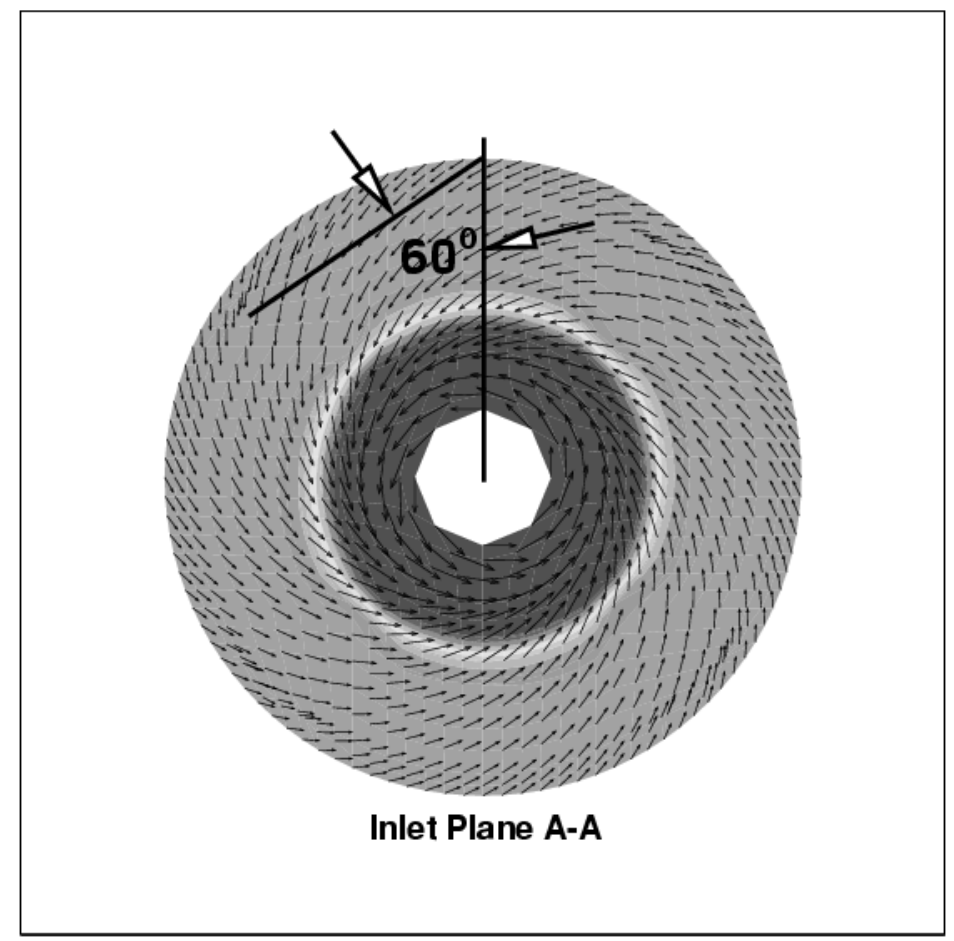

Figure 5.5.4. Swirler Inlet Plane and Vane Angle (CCW) 


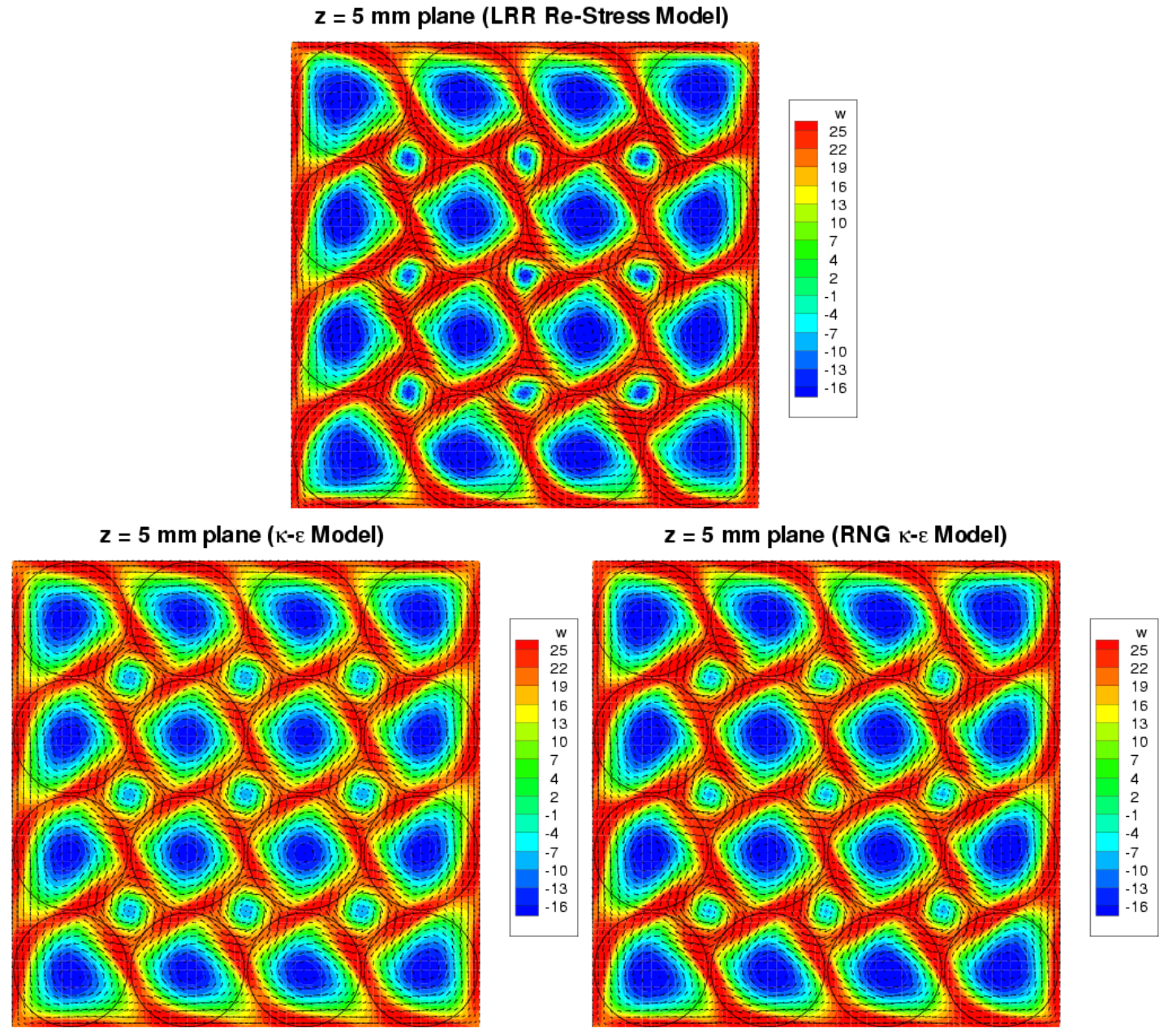

Figure 5.5.5. Axial Velocity Contours with Cross-Flow Vectors at $z=5 \mathrm{~mm}$ 

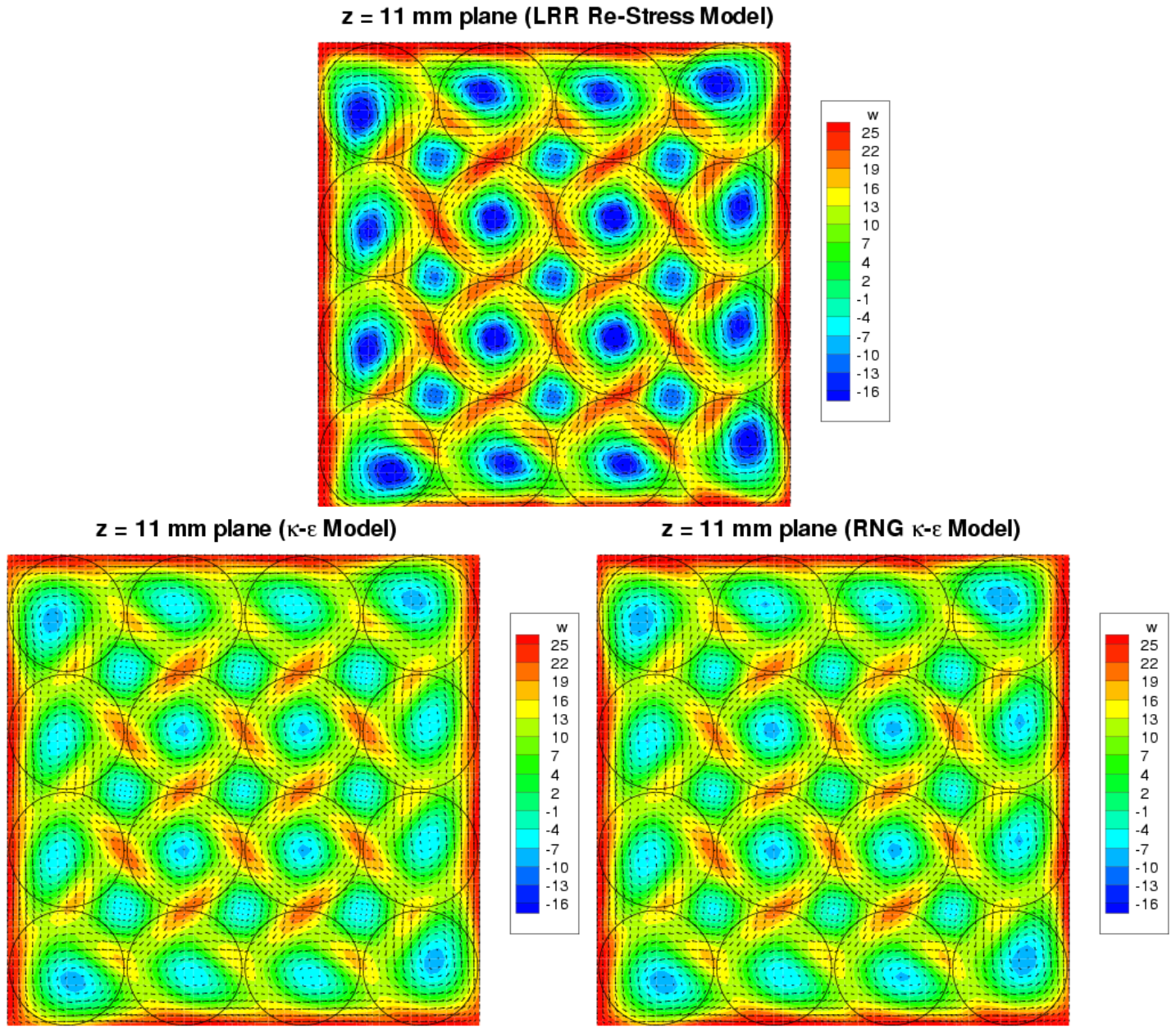

Figure 5.5.6. Axial Velocity Contours with Cross-Flow Vectors at $z=11 \mathrm{~mm}$ 


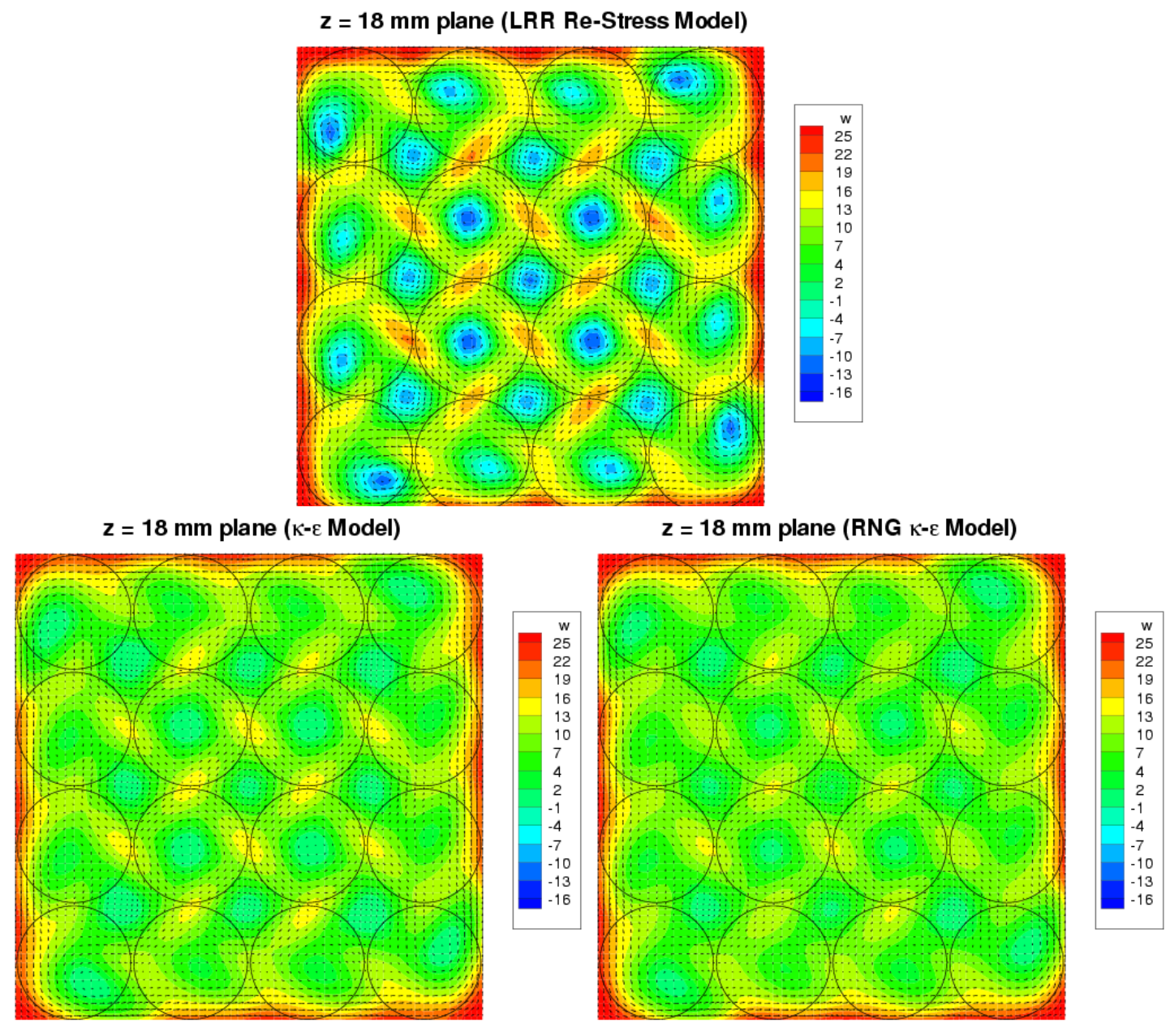

Figure 5.5.7. Axial Velocity Contours with Cross-Flow Vectors at $z=18 \mathrm{~mm}$ 

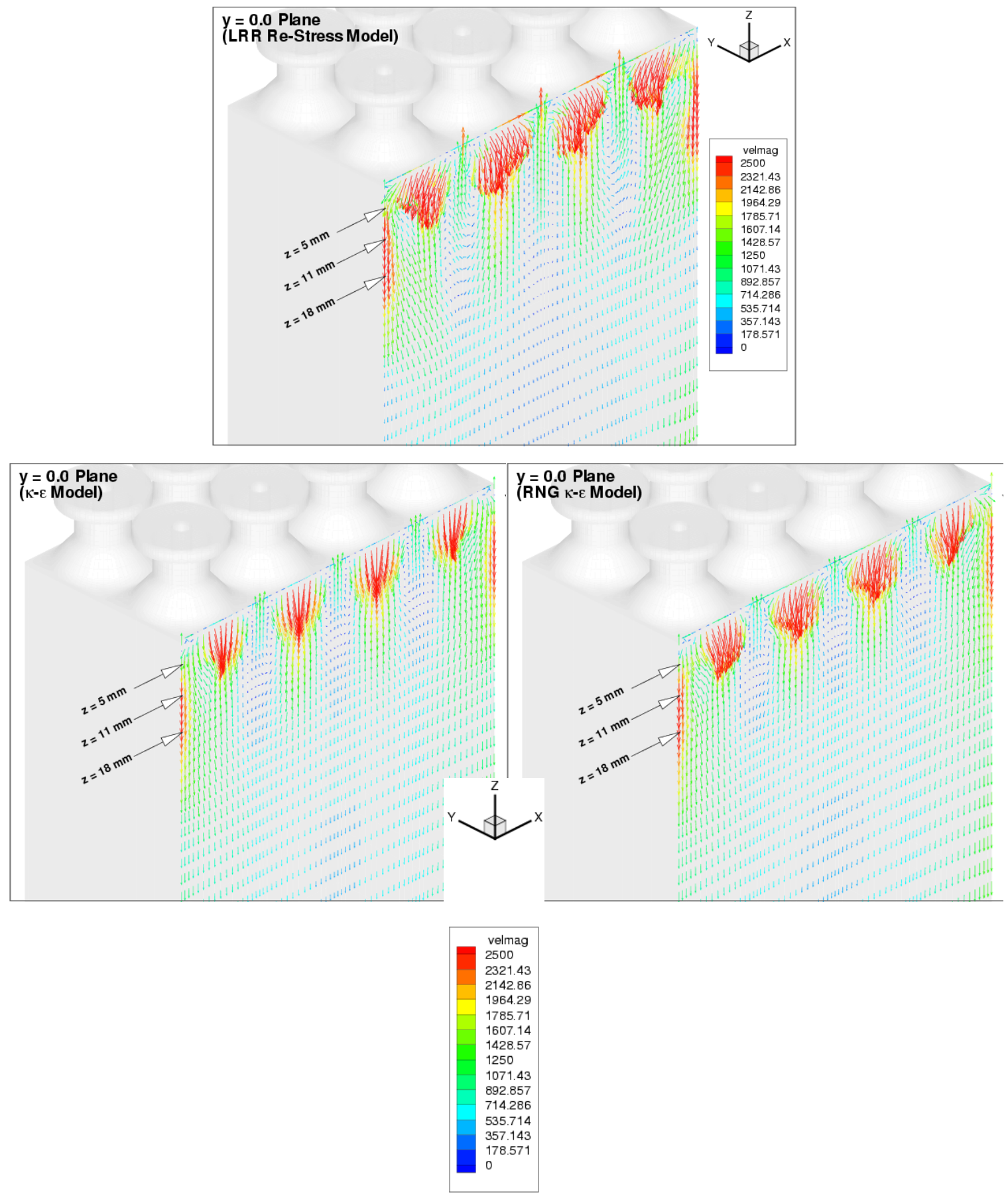

Figure 5.5.8. Velocity Vectors Color-Coded with Magnitude at $y=0 \mathrm{~cm}$ 

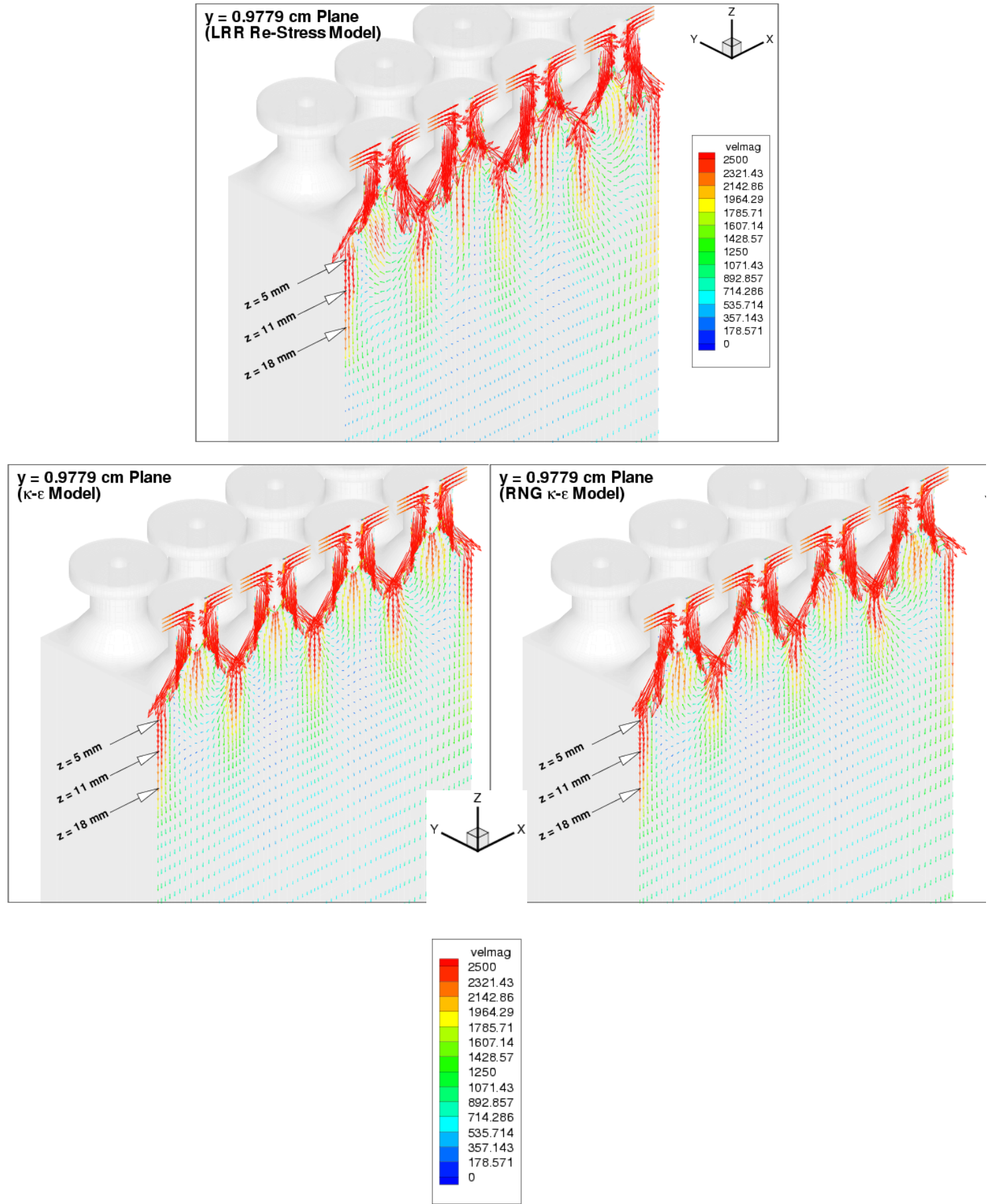

Figure 5.5.9. Velocity Vectors Color-Coded with Magnitude at $y=0.9779 \mathrm{~cm}$ 


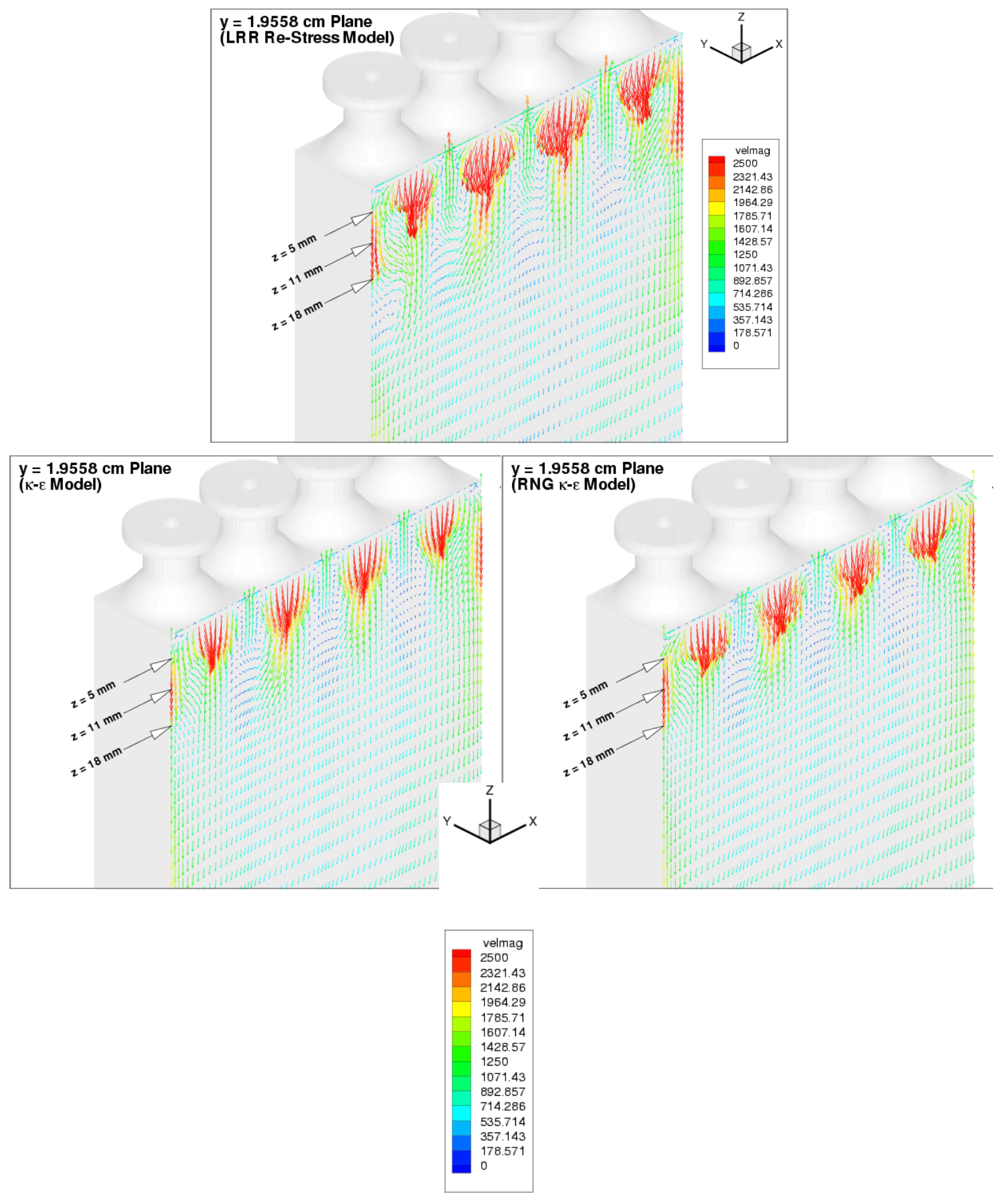

Figure 5.5.10. Velocity Vectors Color-Coded with Magnitude at $y=1.9558 \mathrm{~cm}$ 


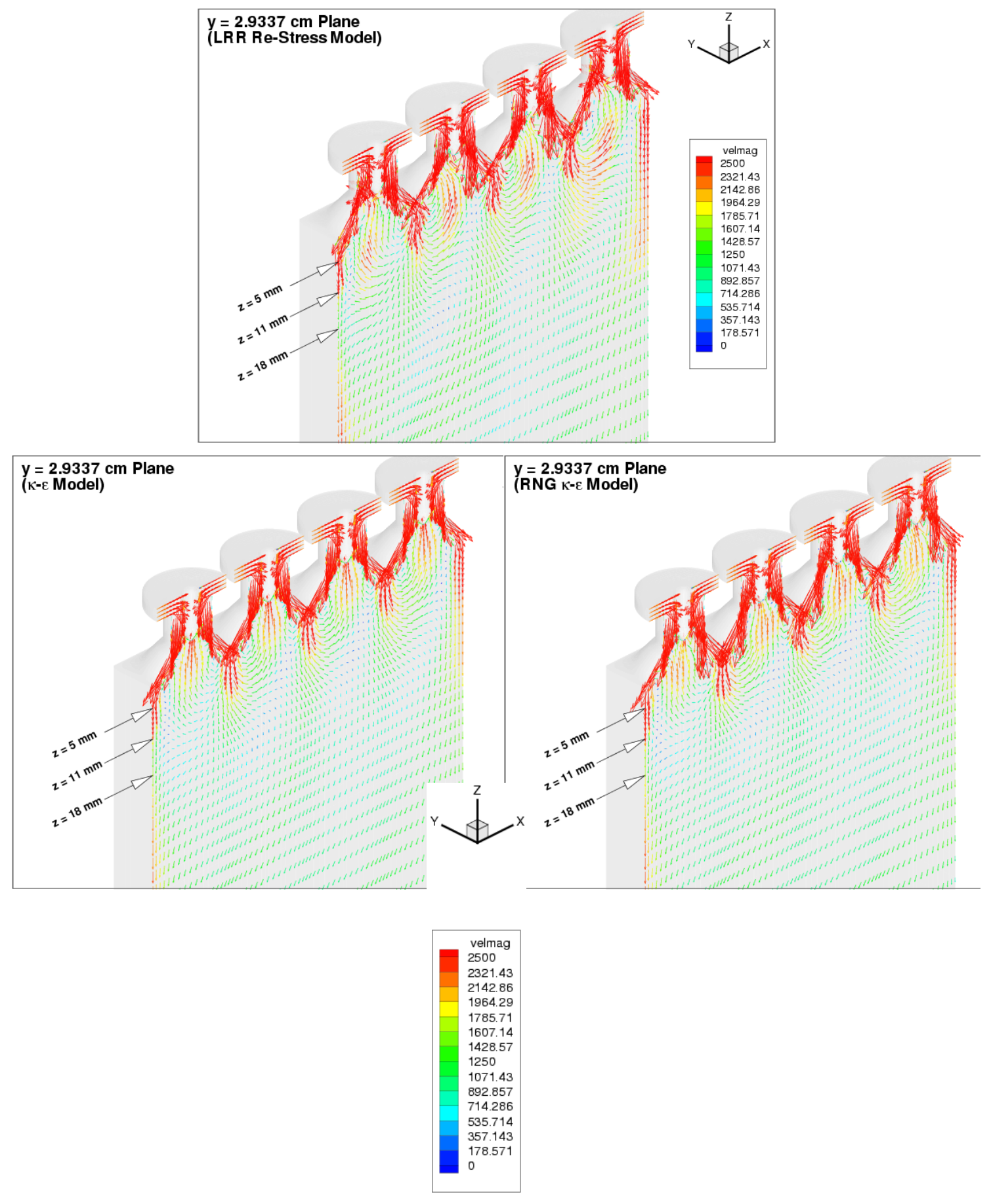

Figure 5.5.11. Velocity Vectors Color-Coded with Magnitude at $y=2.9337 \mathrm{~cm}$ 


\subsection{Design of Radial Air Swirler Blade Lengths}

The effect of blade length on the swirl strength inside a combustor with a radial air swirler is analyzed using computational fluid dynamics (CFD). The objective is to determine the blade length that produces a flow field with the strongest swirl and recirculation for a given swirler blade angle. The air swirler analyzed in this study has fifteen blades, each inclined at $60^{\circ}$ from the radius. The swirler is attached atop a cylindrical combustor. Swirl numbers for various blade lengths are calculated, and the size of the recirculation zones are compared. Both the size of the recirculation zone and swirl number have maximum values at $\mathrm{L} / \mathrm{W}=1.8$, where $\mathrm{L}$ is blade length and $\mathrm{W}$ blade spacing.

\subsubsection{Introduction}

For modern gas-turbine engines, particularly those with lean-direct-injection (LDI) combustors, the design and use of swirlers has a significant impact on engine performance. The strength of swirl, typically characterized by swirl number, is an essential factor in determining fuel-air mixing and flame stability.

A number of studies have pursued the experimental investigation of swirl characteristics inside various combustor/cylinder types with either axial or radial swirlers. For example, Ma et al. [2000] made measurements inside a cylinder attached to a radial swirler with a venturi. In most cases, the tangential velocity retains its magnitude downstream of the swirler and persists to the last measurement station. In some cases, the tangential velocity increases downstream. Similar results were also observed by Rhode et al. [1983] In another study with a similar combustor configuration, Altgeld et al. [1983] found that the tangential velocity approached solid-body rotation near the exit. Crnojevic 
et al. [1999] also reported a similar trend of the swirl velocity. Furthermore, unlike most other studies where swirl number is presented as a scalar quantity for a given flow condition, they showed the dependence of swirl number as a function of axial position as well as the volume flow rate.

Throughout this study, swirl number is used as a parameter that measures the swirl strength for each blade length. Flow with relatively weak swirl, despite having pressure gradient developed in the radial direction, has little, if any, longitudinal (streamwise) pressure gradient. Flow with relatively strong swirl, on the other hand, has a very high degree of radial pressure gradient, creating an adverse pressure gradient in the longitudinal direction. Consequently, the flow reverses its direction locally somewhere downstream, and eventually forms a recirculation region which acts as a flame holder [Gupta et al., 1984]. Therefore, swirl strength is a vital factor in combustor design.

To produce the desired swirl for a given blade angle, the blade must be long enough to deflect the incoming flow. Determination of the blade length is primarily based on a non-dimensional quantity, L/W, where $\mathrm{L}$ is the blade length and $\mathrm{W}$ the spacing between blades. A critical $\mathrm{L} / \mathrm{W}$ value, $(\mathrm{L} / \mathrm{W})_{\text {crit }}$, is defined as the value where every streamline deflects according to the vane angle, and no "see-through" exists as air enters the swirler. As illustrated in Figure 5.6.1, if L, for a given W, is so small that part (or all) of the incoming airflow normal to the swirler circumference can "pass through" without any deflection (Figure 5.6.1a), then weak swirl, if any, will occur downstream. On the contrary, if $\mathrm{L}$ is large enough so that the entire air stream changes its path as it enters the opening (Figure 5.6.1b), then a stronger amount of swirl and stronger recirculation zone will occur 
downstream. From a design point of view, it is desired to have a blade length that produces the optimum amount of swirl and recirculation.

The objective of this study is to use CFD to determine the blade length that produces a flow field with the highest degree of swirl and recirculation corresponding to a given swirler vane angle. The simulation is performed with the CFD code, KIVA-3V [Amsden, 1997], that solves the Reynolds-averaged Navier-Stokes equations with a standard $k$ - $\varepsilon$ turbulence model.

\subsubsection{Swirl Number}

To obtain both qualitative and quantitative comparisons of the results, swirl number, as well as the tangential and axial mean-velocity components, are used to study and identify any significant changes in the flow field brought about by the various blade lengths. Swirl number, defined as the ratio of the tangential to the axial component of the moment of momentum, is expressed in cylindrical coordinates as [Crnojevic et al., 1999]

$$
S=\frac{\int_{A} \rho \vec{r} \times\left(u_{z} u_{\theta}\right) \hat{e}_{\theta} d A}{\int_{A} \rho \vec{r} \times u_{z}^{2} \hat{e}_{z} d A}
$$

where $\rho$ is the fluid density, $r$ the radial distance of a fluid particle from the axis, $u$ the velocity, $A$ the cross-sectional area, and $\hat{e}$ the unit vector. Here, the subscript $z$ represents the streamwise direction, and $\theta$ refers to the azimuthal component. The swirl is then evaluated as a function of the streamwise direction $z$ so that a plot of $S$ vs. $z$ clearly shows the changes in swirl strength across the entire combustor length. 


\subsubsection{Computational Domain and Boundary Conditions}

The geometry begins with a radial air swirler, followed by a straight cylindrical combustor, and ends with a short convergent section. The swirler has a total of fifteen blades along its perimeter. Each blade has a $60^{\circ}$ vane angle, measured from the radius, as illustrated in Figure 5.6.2. The swirler has a diameter of $2 \mathrm{~cm}$, which results in a single air opening, $\mathrm{W}$, of $0.25 \mathrm{~cm}$, based on a diameter-to-W ratio of 8 . The blade length, $\mathrm{L}$, ranges from $0.25 \mathrm{~cm}\left(0.1^{\prime \prime}\right)$ to $0.46 \mathrm{~cm}\left(0.18^{\prime \prime}\right)$. The blade lengths employed in this study are listed in Table 5.6. A $24^{\circ}$ sector mesh containing a single blade, with periodic azimuthal boundaries, is used for the simulation to minimize the computational effort. A fullcylinder mesh is shown in Figure 5.6.3.

A mass flow rate of $9.3 \mathrm{~g} / \mathrm{s}$ is imposed normal to the radial opening of the swirler. The velocity for each case is then adjusted to conform to this mass flow rate. The ambient temperature and pressure are set at the standard atmospheric condition. The pressure drop, therefore, varies slightly from one case to the other. It should be noted that, in reality, experiments are usually carried out such that the pressure drop is held constant, while the inflow area is altered to achieve a constant mass flow rate.

Table 5.6: Blade Length Cases

\begin{tabular}{|c|c|c|c|c|}
\hline Case & $1^{\mathrm{a}}$ & 2 & 3 & 4 \\
\hline $\begin{array}{c}\text { Length, cm } \\
\text { (inch) }\end{array}$ & $\begin{array}{c}0.254 \\
(0.1)\end{array}$ & $\begin{array}{c}0.381 \\
(0.15)\end{array}$ & $\begin{array}{c}0.406 \\
(0.16)\end{array}$ & $\begin{array}{c}0.419 \\
(0.165)\end{array}$ \\
\hline \hline Case & 5 & 6 & 7 & 8 \\
\hline $\begin{array}{c}\text { Length, cm } \\
\text { (inch) }\end{array}$ & $\begin{array}{c}0.432 \\
(0.17)\end{array}$ & $\begin{array}{c}0.445 \\
(0.175)\end{array}$ & $\begin{array}{c}0.450 \\
(0.177)\end{array}$ & $\begin{array}{c}0.457 \\
(0.18)\end{array}$ \\
\hline
\end{tabular}

a. This case corresponds to $(\mathrm{L} / \mathrm{W})_{\text {crit }}$ 


\subsubsection{Results and Discussion}

To show how varying the blade length affects the flow, the size of the central toroidal recirculation zones, or CTRZ, are compared for all blade lengths. Table 5.7 summarizes the CTRZ depths, measured from the swirler exit to the downstream-edge of the CTRZ where the flow begins to recirculate.

Table 5.7: CTRZ Sizes

\begin{tabular}{|c|c|c|c|c|}
\hline Case & 1 & 2 & 3 & 4 \\
\hline CTRZ $(\mathrm{cm})$ & 2.22 & 2.37 & 2.41 & 2.43 \\
\hline \hline Case & 5 & 6 & 7 & 8 \\
\hline CTRZ $(\mathrm{cm})$ & 2.46 & 2.48 & 2.47 & 2.47 \\
\hline
\end{tabular}

It is obvious from Table 5.7 that the size of the recirculation zone reaches a maximum value just short of $2.5 \mathrm{~cm}$ at the blade length corresponding to Case $6(\mathrm{~L} / \mathrm{W}=1.8)$.

Figure 5.6.4 shows the computed swirl numbers versus combustor axial locations. To aid in the explanation, a close-up view of the plot has been inserted. A streamline plot has also been included in the same figure for ease of visualization, particularly for the CTRZ. A general observation from Figure 5.6.4 is that for all blade lengths, the swirl numbers exhibit a similar trend as the flow passes through the combustor. The swirl reduces in strength near the swirler exit, then gradually increases further downstream and continues so to the straight burner outlet, and rapidly decreases inside the convergent section through the outflow boundary.

The trend can be interpreted based on the combustor geometry and flow physics. As air exits the swirler and enters the primary combustion zone, its azimuthal velocity 
components diminish relative to the axial velocity. This is due to the sudden expansion the flow encounters, and the emergence of a CTRZ. This CTRZ is in turn induced by the adverse pressure gradient brought about by the strong swirl. The decrease in swirl strength immediately after the swirler occurs at the expense of weakening tangential momentum as the flow creates a reversed axial pressure gradient and momentum flux. It is noted that, by examining both the $\mathrm{S}$ vs. $\mathrm{z}$ and streamline plots in Figure 5.6.4, the lowest $\mathrm{S}$ values lie in the vicinity of $1 \mathrm{~cm}$ downstream of the swirler, approximately at the center of the CTRZ.

Farther downstream, the swirl strength increases, slowly at first, then rapidly near the burner exit. This increase of swirl number may be from the highly confined burner region. As the diameter of the burner $(2.54 \mathrm{~cm})$ is only slightly larger than that of the swirler $(2 \mathrm{~cm})$, the flow has little space to expand. Instead, it is carried downstream in a relatively narrow passage where the tangential-momentum flux quickly builds up. Finally, the swirl number sharply decreases inside the convergent section through the outflow boundary, as the contracting passage effectively accelerates the flow in the axial direction.

Figure 5.6.5 shows the swirl numbers for all cases at $\mathrm{z}=2.5 \mathrm{~cm}$. An important observation from the plot is the rate at which the swirl number increases as the blade becomes longer. As blade length increases, the corresponding swirl number increases with a smaller increment, reaching a maximum value. In fact, the swirl levels for Cases 7 and 8 , the two longest blades, are lower than that for Case 6. This may be caused by the "pinching" effect between the neighboring blade tips, where the blades extend so deep into the radius that the flow path becomes obstructed, thus reducing the swirl strength.

The velocity plots in Figure 5.6.6 show that the flow exhibits a behavior characteristic of a forced vortex. In particular, the tangential velocity plot in Figure 5.6.6b 
indicates that the swirling flow develops quickly after the swirler and maintains a consistent radial profile throughout the entire combustor. The flow approaches that of solid-body rotation near the axis, and the vortex core extends substantially toward the combustor outer radius.

\subsubsection{Conclusion}

A numerical study was conducted to investigate the behavior of swirl inside a combustor with a radial swirler. In particular, the sensitivity of the swirl strength and the length of the recirculation zone to the swirler blade length were examined. The goal was to determine the blade length that induces the maximum amount of swirl and recirculation for the given geometry and flow conditions. Eight blade-length cases were analyzed for this study. The size of the central toroidal recirculation zone for all blade lengths were compared. The result shows that the recirculation zone reaches a maximum at a value of $\mathrm{L} / \mathrm{W}=1.8$. Furthermore, a dimensionless parameter, swirl number, $\mathrm{S}$, written as a function of the streamwise coordinate, was used to characterize the swirling flow. The results show that, for any given axial location, the swirl generally increases in strength with increasing blade length, but at a decreasing rate until a maximum is reached, with a further increase in blade length giving lower swirl. For example, the swirl numbers of the two longest blades, Case 7 and Case 8, are lower than that of the shorter blade for Case 6. Hence, the blade length of Case $6(\mathrm{~L} / \mathrm{W}=1.8)$ appears to be the optimum length that induces the maximum amount of swirl and recirculation for the given geometry and inlet conditions. 


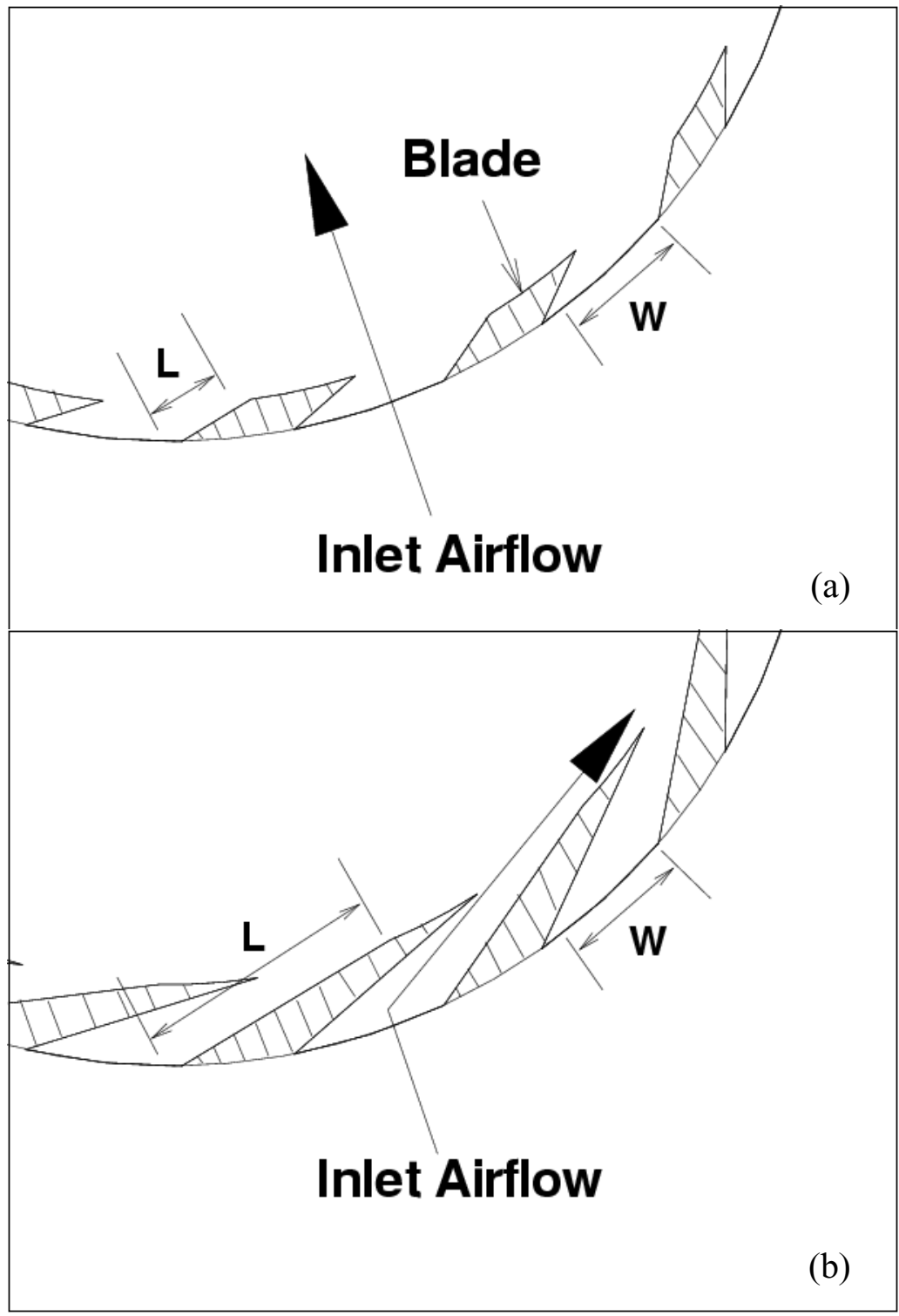

Figure 5.6.1. Illustration of Blade Length Effect on Air Flow:

(a) $\mathrm{L} / \mathrm{W}<(\mathrm{L} / \mathrm{W})_{\text {crit }}$, (b) $\mathrm{L} / \mathrm{W}>(\mathrm{L} / \mathrm{W})_{\text {crit }}$ 


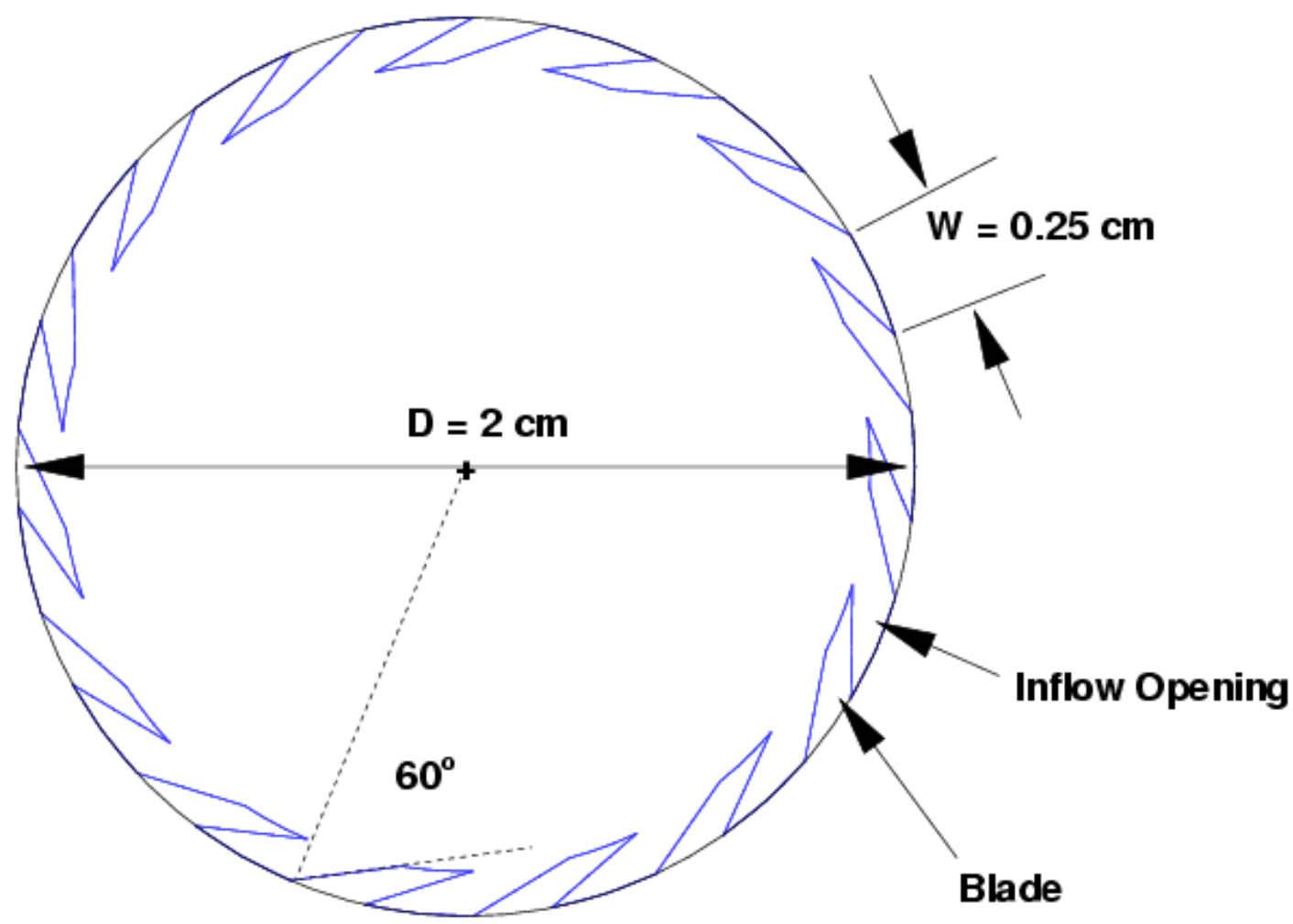

Figure 5.6.2. Swirler Geometry (Case 1)

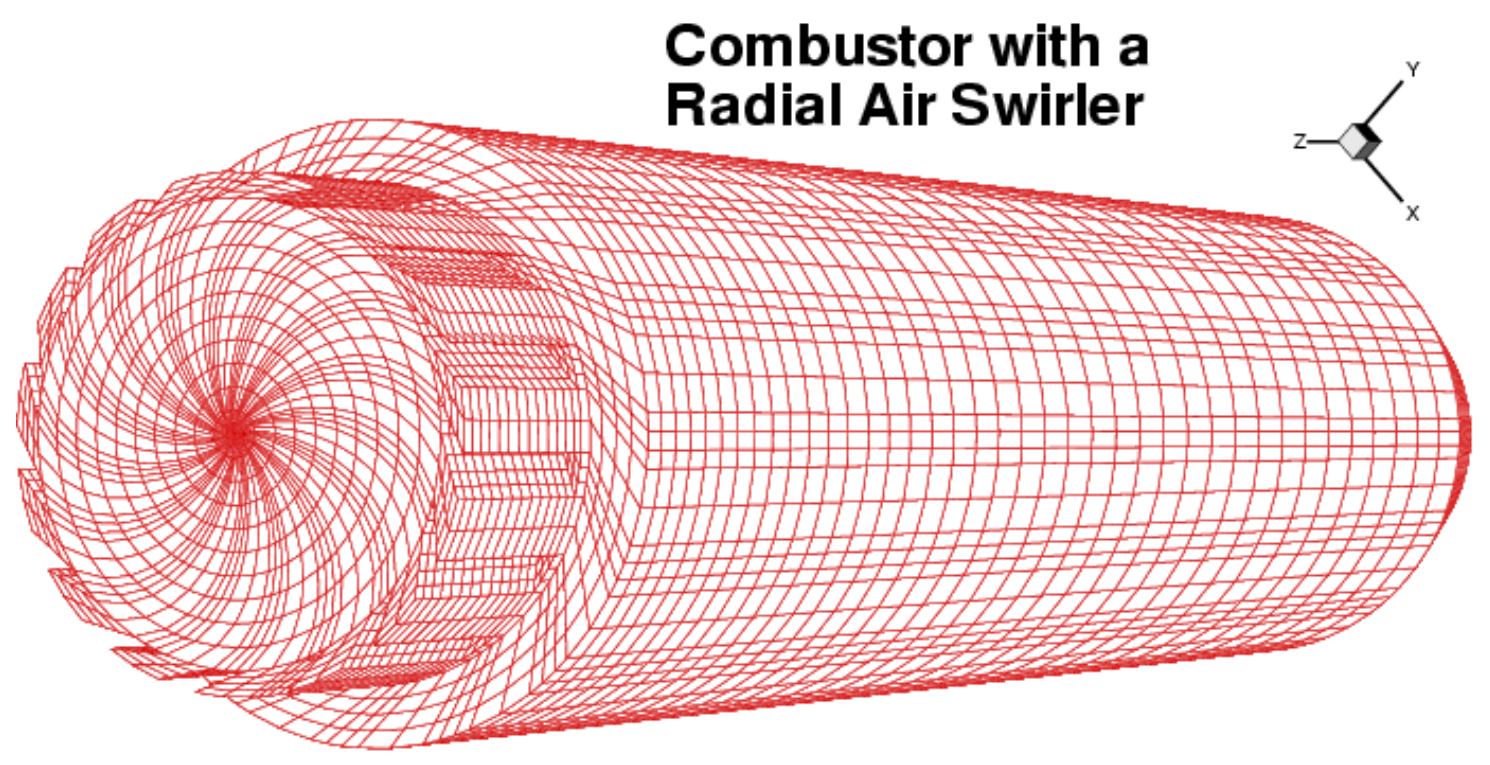

Figure 5.6.3. Computational Mesh of the Combustor with a Radial Air Swirler 

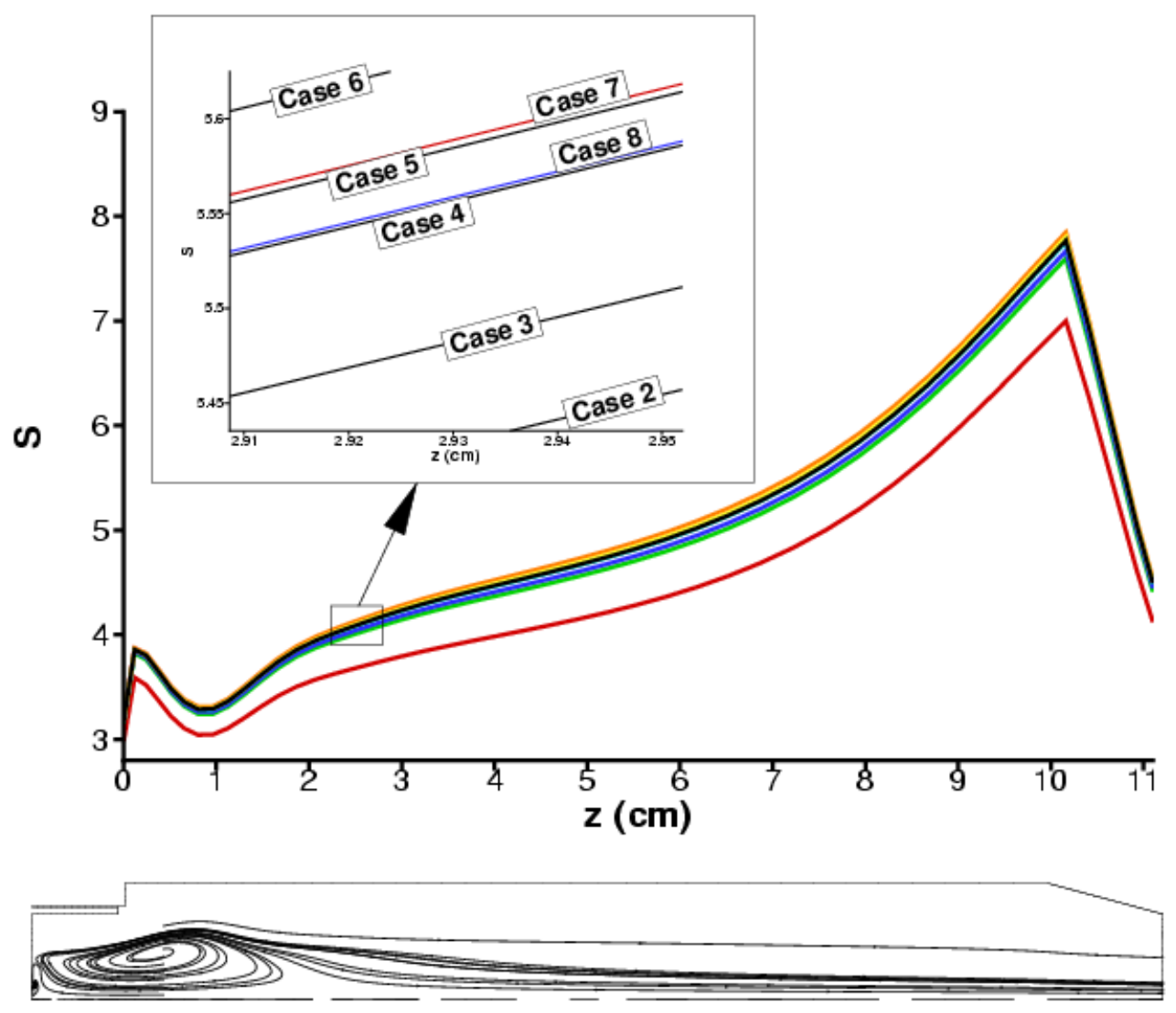

Figure 5.6.4. 4 Swirl Number vs. Axial Locations

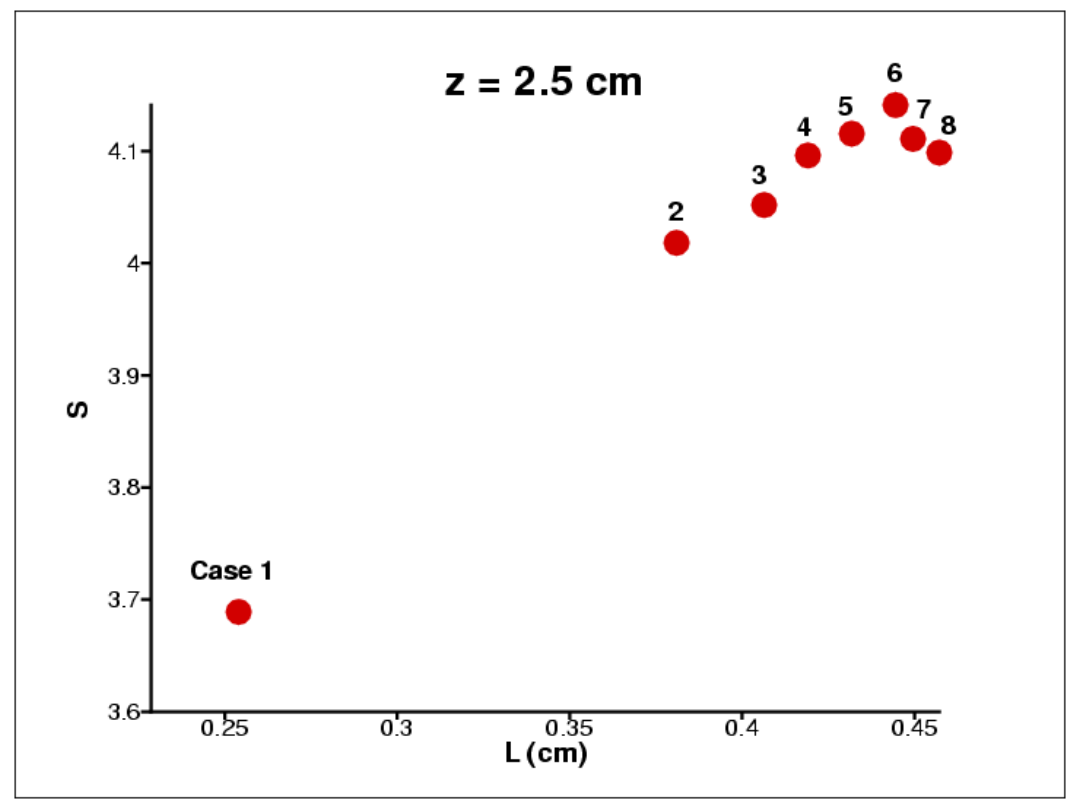

Figure 5.6.5. Swirl Number for All Blade Lengths at $\mathrm{z}=2.5 \mathrm{~cm}$ 
Axial Velocity $U_{z}$

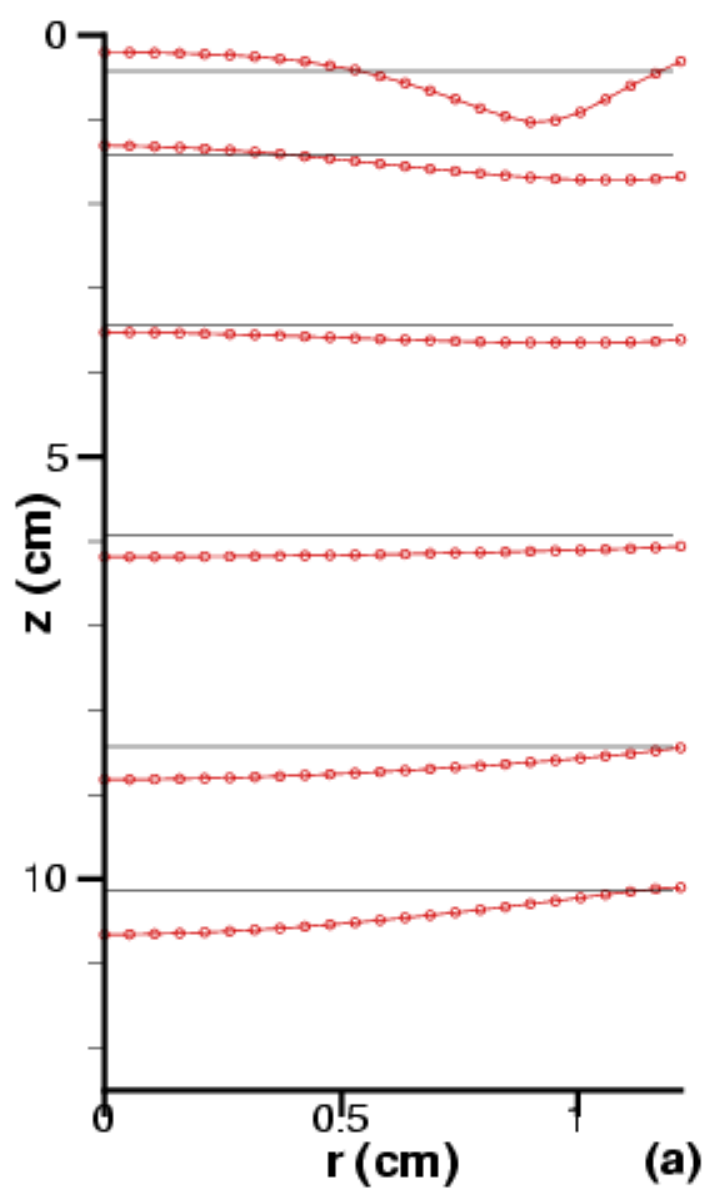

Tangential Velocity $U_{\theta}$

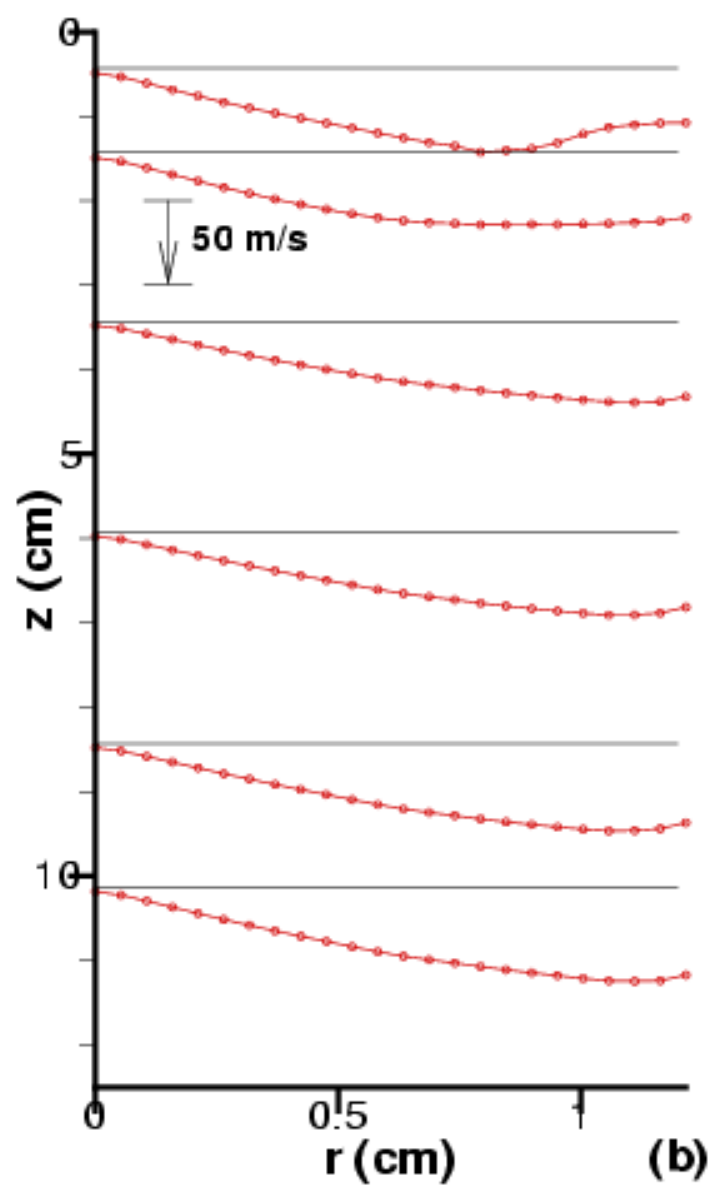

Figure 5.6.6. Radial Profiles of Mean-Flow Velocity for Case $6(\mathrm{~L}=0.445 \mathrm{~cm})$ :

(a) Axial, (b) Tangential 


\section{Chapter 6}

\section{Other Contributions}

\subsection{Total-Variation Diminishing Scheme for the Convective Transport}

Due to the presence of walls and complex geometry, and hence large gradients in the flow field, a high-resolution, spatially second-order, symmetric Total Variation Diminishing (TVD) scheme has been incorporated into KIVA, as an attempt to improve the solution of the momentum convection. A particular form of the scheme, to be discussed below, was implemented as an added feature to KIVA's original schemes. The user can choose among the methods according to the need of accuracy and computational overhead.

High-resolution and higher-order TVD schemes are known to possess the ability to capture and resolve high velocity gradients as well as discontinuities in the flow field. 
Under the constraint of the scheme, the total variation of the solution does not increase with time. This reinforces a physically reasonable solution [Anderson, 1995]. The total variation is defined as

$$
T V(u)=\int_{-\infty}^{\infty}\left|\frac{\partial u}{\partial x}\right| d x
$$

In discrete form of the expression, the total variation for a scalar conservation form of equation can be written as

$$
T V\left(u^{n}\right)=\sum_{-\infty}^{\infty}\left|u_{i+1}^{n}-u_{i}^{n}\right|
$$

Thus, all TVD schemes must satisfy the condition

$$
T V\left(u^{n+1}\right)<T V\left(u^{n}\right)
$$

There are a variety of TVD schemes developed by a number of researchers such as Yee [1987], Davis [1984], Roe [1984], and Osher and Chakravarthy [1986]. Previous studies,

e.g., Yang et al. [1994], Siow [1999] and Siow et al. [2000] have shown improvements achieved by the use of TVD.

The implementation of TVD in the KIVA code will now be discussed.

\subsubsection{Implementing TVD in KIVA}

Two differencing schemes are originally available in the KIVA code for the convection calculations in Phase C, namely, partial donor cell (PDC) differencing and quasi-second-order upwind (QSOU) differencing methods. The partial donor cell method is monotonic, but it is only first-order accurate in space. The quasi-second-order scheme was devised to achieve reasonable accuracy while maintaining monotonicity. The method 
is second-order accurate in space only if the gradient of a scalar function $f$ is a constant in the entire flow domain, and nearly second-order accurate when the gradient varies slowly [Amsden et al., 1989].

The TVD scheme that has been implemented in KIVA is a symmetric, spatially second-order formulation. It is based on a scheme proposed by Yee [1987], modified to be used in consistency with KIVA's physical and computational structures. In KIVA, the computation of convective transport is performed in Phase C. The flow field is frozen in time, and the computational mesh is remapped to the new coordinates - be it a new location in the case of a moving mesh (e.g., internal combustion engines), or the same location as the previous time-step in the case of a stationary grid (e.g., gas turbine combustors). The rezone/remap phase (i.e., Phase C) procedure in the KIVA code is summarized in Appendix C.

The variables to be fluxed, $f$, include the species mass $m_{\text {species }}$, species density $\rho_{\text {species }}$, specific internal energy $I$, turbulence kinetic energy $k$, Reynolds-stresses $u_{i} u_{j}$, turbulence length scale $l_{T}$, and each of the velocity components $u, v, w$; i.e.,

$$
f=m_{\text {species }}, \rho_{\text {species }}, I, k, u_{i} u_{j}, l_{T}, u, v, w
$$

A simple comparison of one-dimensional convection among several schemes is presented in Figure 6.1, c.f. Amsden et al. [1989]. The dots represent the cell-centered density for each cell. The density values have large variation from one cell to the next, so as to demonstrate the characteristics of each convection scheme. 


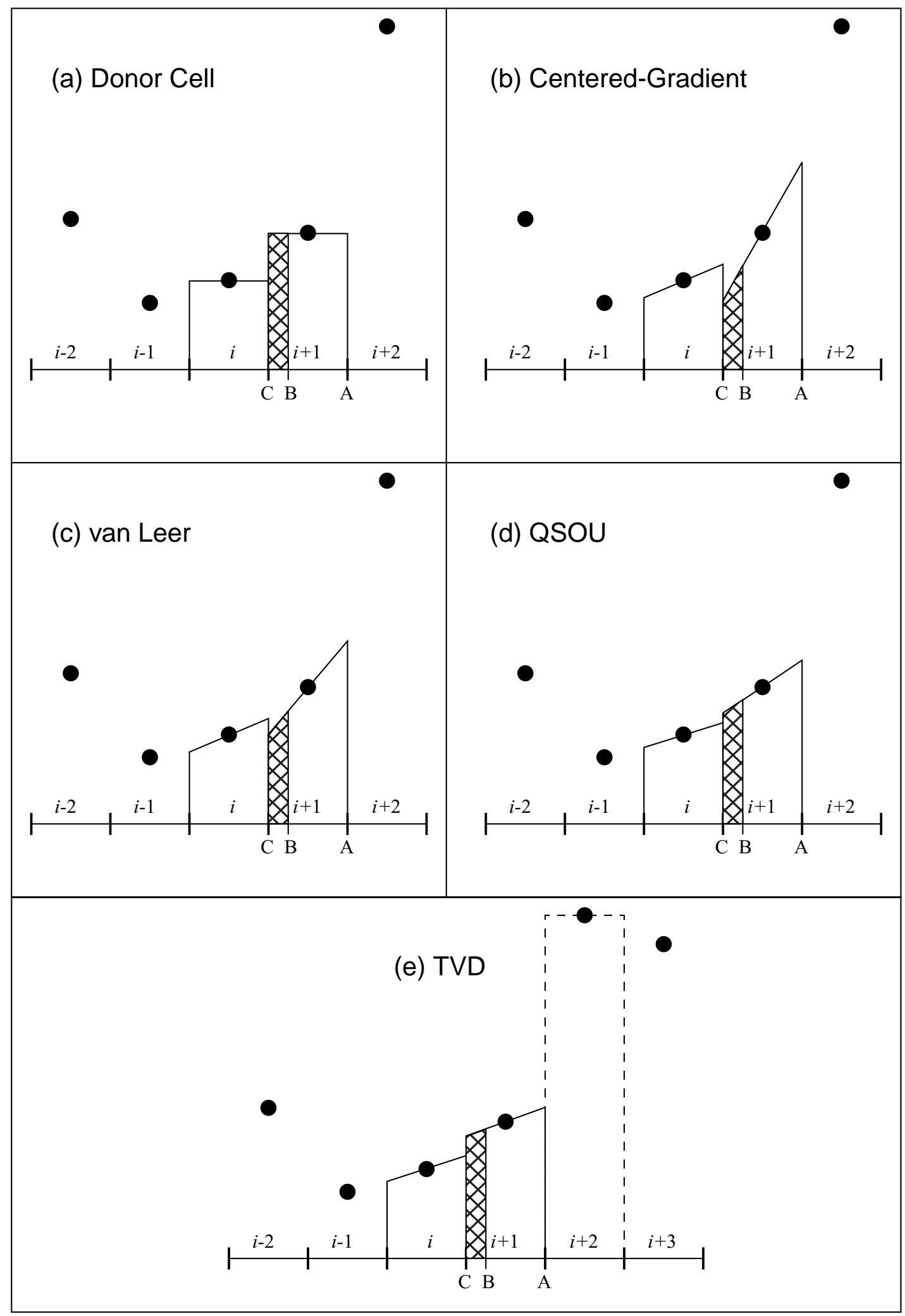

Fig. 6.1. Density Profiles For Five Convection Schemes 
The density profile is assumed to be linear within the cell. In this example, the right boundary of cell $i+1$ moves from the old-time position A to the new-time position $\mathrm{B}$, while its left boundary (i.e., point C) remains stationary. The amount of mass convected due to this A-B boundary movement is equal to the area under the slope between A and B. Accordingly, the new-time total mass in a cell is the area under the same (old-time) slope but between the new boundaries, B and C, as represented by the crosshatched area.

The donor cell method is strongly monotone but suffers from large numerical diffusion, hence compromising accuracy. The centered-gradient scheme is second-order accurate and less diffusive, but can have large overshoots or undershoots in the neighborhood where large gradients exist. As a result, the centered-gradient method is not a monotone scheme. The van Leer scheme improves on the monotonicity, although it is only weakly monotone at best. The QSOU scheme is strongly monotone, although its accuracy is highly sensitive to the variation of the density gradient on the mesh.

The TVD scheme can be shown to satisfy the monotone condition [Yee, 1987]. Its accuracy is maintained at second-order except when local extrema are encountered (e.g., across a shock wave), where the accuracy reduces to first-order. The particular TVD scheme adopted for this dissertation is based on the five-point Yee-Roe-Davis symmetric scheme, of which further detail can be found in Yee [1987] and references therein.

The flux-limiting terms, which delineate the slope of the convected flux, is now described for cell-centered quantities. The function of the flux limiters in a TVD scheme is to insert control over the gradients of density (or any quantity being convected).

The Yee-Roe-Davis scheme is a five-point technique, i.e., it uses a total of five cells to calculate the value of the fluxes. In KIVA, however, fluxes are to be sought across a cell 
face. Therefore, modifications have been made such that six points are involved in the procedure, although five points are used for the actual calculations. The density used for fluxing across a cell face $a$, during each subcycle $v$, is

$$
\rho_{a}^{v}=\left\{\begin{array}{c}
\rho_{i}+\frac{1}{2}\left(Q_{a+\frac{1}{2}}^{\prime}-Q_{a-\frac{1}{2}}^{\prime}\right)\left|x_{a}-x_{i}^{c}\right|\left(1-\frac{\delta V_{a}}{V_{i}^{v}}\right), \delta V_{a}>0 \\
\rho_{i+1}-\frac{1}{2}\left(Q_{a+\frac{1}{2}}^{\prime \prime}-Q_{a-\frac{1}{2}}^{\prime \prime}\right)\left|x_{a}-x_{i+1}^{c}\right|\left(1+\frac{\delta V_{a}}{V_{i+1}^{v}}\right), \delta V_{a}<0
\end{array}\right.
$$

In Eq.(6.5), $x_{a}$ is the new-time location of the cell face $a$, and $x_{i}^{c}$ is the new-time cellcentered coordinates of cell $i$. The quantity $V_{i}^{v}$ is the cell volume after $v$ subcycles, for which the equation can be found in Appendix C. $\delta V_{a}$ is the flux volume due to the movement of cell face $a$.

The quantities $Q^{\prime}$ and $Q^{\prime \prime}$ are the limiter terms. Several choices are available, all of which employ the "minmod" function, defined as

$$
\begin{aligned}
\operatorname{minmod}(x, y) & =\operatorname{sgn}(x) \cdot \max \{0, \min [|x|, y \cdot \operatorname{sgn}(x)]\} \\
& =\left\{\begin{array}{l}
x, \text { if }|x|<|y| \text { and } x y>0 \\
y, \text { if }|x|>|y| \text { and } x y>0 \\
0, \text { if } x y<0
\end{array}\right.
\end{aligned}
$$

The function $\operatorname{minmod}(x, y, z)$ is similarly defined. For this dissertation, the limiters $Q$ are chosen to be

$$
\begin{aligned}
& Q_{a+\frac{1}{2}}^{\prime}=\operatorname{minmod}\left(\left.\frac{\Delta \rho}{\Delta x}\right|_{i-\frac{1}{2}},\left.\frac{\Delta \rho}{\Delta x}\right|_{i+\frac{1}{2}},\left.\frac{\Delta \rho}{\Delta x}\right|_{i+\frac{3}{2}}\right) \\
& Q_{a-\frac{1}{2}}^{\prime}=\operatorname{minmod}\left(\left.\frac{\Delta \rho}{\Delta x}\right|_{i-\frac{3}{2}},\left.\frac{\Delta \rho}{\Delta x}\right|_{i-\frac{1}{2}},\left.\frac{\Delta \rho}{\Delta x}\right|_{i+\frac{1}{2}}\right)
\end{aligned}
$$




$$
Q_{a+\frac{1}{2}}^{\prime \prime}=\operatorname{minmod}\left(\left.\frac{\Delta \rho}{\Delta x}\right|_{i+\frac{1}{2}},\left.\frac{\Delta \rho}{\Delta x}\right|_{i+\frac{3}{2}},\left.\frac{\Delta \rho}{\Delta x}\right|_{i+\frac{5}{2}}\right)
$$

and

$$
Q_{a-\frac{1}{2}}^{\prime \prime}=\operatorname{minmod}\left(\left.\frac{\Delta \rho}{\Delta x}\right|_{i-\frac{1}{2}},\left.\frac{\Delta \rho}{\Delta x}\right|_{i+\frac{1}{2}},\left.\frac{\Delta \rho}{\Delta x}\right|_{i+\frac{3}{2}}\right)
$$

The subscript accompanying each density gradient term represents the mid-point between two cells, e.g.,

$$
\left.\frac{\Delta \rho}{\Delta x}\right|_{i+\frac{1}{2}}=\frac{\rho_{i+1}-\rho_{i}}{x_{i+1}^{c}-x_{i}^{c}}
$$

Figure 6.2 shows a schematic of one-dimensional cells and cell-centered points needed during the convection calculations.

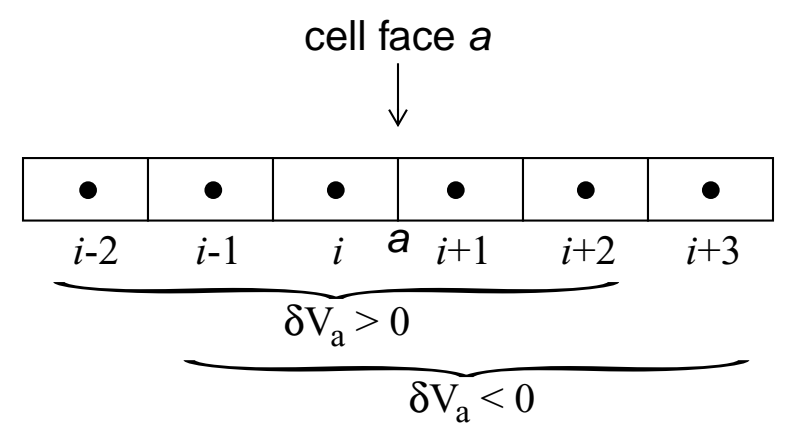

Fig. 6.2. Cells Used in TVD Calculations of Convective Flux

Similar to the QSOU formulation in KIVA, when a boundary is encountered, the gradient in the direction normal to the wall is automatically set to zero. For vertexcentered quantities, i.e., the velocity components $u, v$, and $w$, similar procedure compatible with KIVA's structure is carried out.

All coding modifications were performed in subroutines $\mathrm{ccflux}$, momflx, as well as rsmcflx of the Reynolds-stress turbulence model. 


\subsection{Reduced Kinetic Mechanism}

A 16-species, 23-step chemical kinetic mechanism [Kundu et al., 1998] has been incorporated into the KIVA code, significantly improving the ease of inputting species and reaction steps, as well as the accuracy in predicting the production of $\mathrm{NO}_{\mathrm{x}}, \mathrm{CO}, \mathrm{CO}_{2}$ and hydrocarbons.

A description of the mechanism has been included in Chapter 5.2, and detail of the reaction steps and coefficients can be found in Appendix B.

\subsubsection{Implementation in KIVA}

The implementation of the kinetic mechanism in KIVA is relatively straightforward. The most significant improvement over the original KIVA code is the input interface. Instead of having to input all necessary thermodynamic data in the input file itape 5, the species properties, reaction steps, and coefficients are now grouped into a separate file called mechanism. Each reaction step is expressed and formatted in a symbolic fashion. This results in much simpler and more legible and descriptive input procedure.

The contents of the file mechanism can be found in Appendix B.

The major subroutine modified for the new mechanism is rinput. A new subroutine has been created to accommodate the calculations of species densities and internal energy using the data in mechanism. The original subroutine chem is therefore bypassed.

In addition to facilitating ease of input, the modification also uses polynomial curve-fitting for the enthalpy data, instead of searching through the limited 
thermodynamic tables supplied in KIVA. This allows for a more robust and efficient method to retrieve species properties. The user may also replace the existing curve-fit equations or include additional property data with relative ease, and without the need to manually include individual property values at discrete intervals. 


\section{Chapter 7}

\section{Conclusions}

The Reynolds-stress turbulence model has been successfully incorporated into the KIVA code for engine simulations. Validation is accomplished by comparisons of computed results with experimental and DNS data for plane channel flow and axisymmetric pipe flow. The RSTM has been applied to numerous engine flow calculations, including various gas turbine combustors and internal combustion engines. In all applications where experimental data are available, the RSTM results agree favorably to the measurements, and it outperforms the two-equation turbulence models

including the standard $k-\varepsilon$, RNG-variant $k-\varepsilon$, as well as the cubic nonlinear $k-\varepsilon$ in the National Combustion Code. 
Within the framework of the Reynolds-averaged Navier-Stokes approach, the RSTM represents the highest level and most accurate modeling technique. Unlike the popular eddy-viscosity models such as the standard $k-\varepsilon$ and $k-\omega$, the RSTM directly solves for the Reynolds-stress tensors, of which each component is prescribed and solved for by an individual partial differential equation. The advantages are obvious as well as important. Unlike eddy-viscosity turbulence models, no ad hoc modifications are necessary when RSTM is used. Solving for each Reynolds stress also allows for better resolution of flow details, separation, swirl, secondary motion, etc.

The additional equations inevitably result in longer turnaround time. However, the increase is only marginal. In the study of the LDI combustor with axial swirlers, for example, $12 \%$ difference in CPU time is observed between the RSTM and the standard $k$ - $\varepsilon$ model under identical flow conditions and with the same computational mesh.

Other more sophisticated methods, such as Direct Numerical Simulation and Large Eddy Simulation, are also being developed. These techniques are conceptually the most accurate approach to study turbulent flows, since modeling is not required (in DNS) or is necessary only in regions of the smallest scales (in LES). However, these techniques have not yet been able to reach a wide applicability, particularly in high-Reynolds-number flows.

As a contribution to the literature, the research work conducted for this dissertation has resulted in a number of journal publications and conference proceedings, of which several select cases have been presented in Chapter 5 . 
In conclusion, the Reynolds-stress turbulence model has been proven to be an appropriate choice for engine simulations, for its robustness, consistency, applicability, and relatively low computational efforts for present-day machines. 


\section{REFERENCES}

Altgeld, H., Jones, W. P., and Wilhelmi, J., "Velocity Measurements in a Confined Swirl Driven Recirculating Flow,” Experiments in Fluids, Vol. 1, pp. 73-78, 1983.

Amsden, A. A., O’Rourke, P. J., and Butler, T. D., “KIVA-II: A Computer Program for Chemically Reactive Flows with Sprays," Loas Alamos National Laboratory, LA11560-MS, 1989.

Amsden, A.A., “KIVA-3: A KIVA Program with Block-Structured Mesh for Complex Geometries," Los Alamos National Laboratory, LA-12503-MS, 1993.

Amsden, A. A., "KIVA-3V: A Block-Structured KIVA Program for Engines With Vertical or Canted Valves," Los Alamos National Laboratory, LA-13313-MS, Los Alamos, NM, 1997.

Amsden, A. A., “KIVA-3V, Release 2, Improvements to KIVA-3V,” Los Alamos National Laboratory, LA-13608-MS, 1999.

Anderson, D. A., "Effects of Equivalence Ratio and Dwell Time on Exhaust Emissions from an Experimental Premixing Prevaporizing Burner," Report No. NASA TMX71592, 1975.

Anderson, J. D., "Computational Fluid Dynamics: The Basics with Applications," 1st ed., McGraw-Hill, New York, 1995, pp. 499-510.

Bachalo, W. D. and Houser, M. J., "Phase/Doppler Spray Analyzer for Simultaneous Measurements of Drop Size and Velocity Distributions," Optical Engineering, Vol. 23, No. 5, pp. 583-590, 1984. 
Basara, B., "A Numerical Study into the Effects of Turbulent Flows Around Full-Scale Buildings,” Ph.D. thesis, City University, London, U.K., 1993.

Bittker, D.A., “GLSENS, A Generalized Extension of LSENS Including Global reactions and Added Sensitivity Analysis for the Perfectly Stirred Reactor," NASA Reference Publication 1362, 1996.

Bulzan, D. L., "Structure of a Swirl-Stabilized Combusting Spray," Journal of Propulsion and Power, Vol. 11, No. 6, pp. 1093-1102, 1995.

Cline, M. C., Micklow, G. J., Yang, S. L., and Nguyen, H. L., "Numerical Analysis of the Flow-Fields in a Staged Gas Turbine Combustor,' J. of Propulsion and Power, Vol. 111, No. 5, pp. 894-898, 1995.

Cline and Yang, Private Conversation, 1996.

Coordinating Research Council, "Handbook of Aviation Fuel Properties," CRC Report No. 530, 1983.

Cowell, L. H. and Smith, K. O., "Development of a Liquid-Fueled, Lean-Premixed Gas Turbine Combustor," Journal of Engineering for Gas Turbines and Power, Vol. 115, No. 3, pp. 554- 562, 1993.

Craft, T. J., Launder, B. E., and Suga, K., "Extending the Applicability of Eddy Viscosity Models through the Use of Deformation Invariations and Non-Linear Elements," $5^{\text {th }}$ IAHR Conference on Refined-Flow Modeling and Turbulence Measurement, Paris, September 7-10, 1993.

Crandall, M. G. and Majda, A., "Monotone Difference Approximations for Scalar Conservation Laws," Mathematics of Computation, Vol. 34, No. 149, pp. 1-21, 1980. 
Crnojevic, C., Decool, F., and Florent, P., "Swirl Measurements in a Motor Cylinder," Experiments in Fluids, Vol. 26, pp. 542-548, 1999.

Daly, B. J. and Harlow, F. H., "Transport Equations in Turbulence,” Physics of Fluids, Vol. 13, No. 11, pp. 2634-2649, 1970.

Davis, S. F., "TVD Finite Difference Schemes and Artificial Viscosity," ICASE Report No. 84-20, 1984.

Edelman, R.B., and Fortune, O.F., "A Quasi-Global Chemical Kinetics Model for Finite rate Combustion of Hydrocarbon Fuels with Application to Turbulent Burning and Mixing in Hypersonic Engines and Nozzles," AIAA 69-86, 1969.

Fenimore, C.P., "Formation of Nitric Oxide in Premixed Hydrocarbon Flames," Thirteenth Symposium (International) of Combustion, The Combustion Institute, pp. 373$379,1971$.

Galperin, B. and Orszag, S. A., "Large Eddy Simulation of Complex Engineering and Geophysical Flows," Cambridge University Press, Cambridge, U. K., 1993.

Gibson, M. M. and Launder, B. E., "Ground Effects on Pressure Fluctuations in the Atmospheric Boundary Layers," Journal of Fluid Mechanics, Vol. 86, pp. 491-551, 1978.

Gran, I. R., Ertesvag, I. S., Magnussen, B. F., "Influence of Turbulence Modeling of Predictions of Turbulent Combustion," AIAA Journal, Vol. 35, No. 1, pp. 106-110, 1997.

Gridgen CFD and FEA Pre-Processing, V.13.3, Pointwise Inc.

Gupta, A. K., Lilley, D. G., and Syred, N., "Swirl Flows," Abacus Press, Tunbridge Wells, England, 1984. 
Hadzic, I., "Second-Moment Closure Modelling of Transitional and Unsteady Turbulent Flows," Ph.D Dissertation, Delft University of Technology, The Netherlands, 1999.

Han, Z. and Reitz, R. D., “Turbulence Modeling of Internal Combustion Engines Using RNG k-ع Models,” Combust. Sci. and Tech., Vol. 106, pp. 267-295, 1995.

Hanjalic, K., "CFD and Advanced Turbulence Models: Reconciling the Unreconcilable," Invited Lecture, Third International FIRE User Meeting, Graz, Austria, June 16$17,1997$.

Hanjalic, K., "Second-moment Turbulence Closures for CFD: Needs and Prospects," International Journal of Computational Fluid Dynamics, Vol. 12, pp. 67-97, 1999.

Hanjalic, K. and Launder, B. E., "A Reynolds Stress Model of Turbulence and Its Application to Thin Shear Flows," J. Fluid Mechanics, Vol. 52, Part 4, pp. 609638, 1972.

Hanjalic, K. and Launder, B. E., “Contribution Towards a Reynolds-Stress Closure for Low-Reynolds-Number Turbulence," J. Fluid Mechanics, Vol. 74, Part 4, pp. 593$610,1976$.

Harten, A., Hyman J. M., and Lax, P. D., “On Finite-Difference Approximations and Entropy Conditions for Shocks," Communications on Pure and Applied Mathematics., Vol. 29, pp. 297-322.

Hascher, H. G., Schock, H. J., Avanessian, O., and Novak, J., “A Comparision of Modeled and Measured 3-D In-Cylinder Charge Motion Throughout the Displacement of a Four-Valve SI Engine,’ SAE Journal, SAE-2000-01-2799, 2000. 
Hirt, C. W., Amsden, A. A., and Cook, J. L., "An Arbitrary Lagrangian-Eulerian Computing Method for All Flow Speeds" J. Computational Physics, Vol. 14, pp. 227-253, 1974.

Hoffmann, K. A. and Chiang, S. T., “Computational Fluid Dynamics for Engineers Volume 1," Engineering Education System, Wichita, Kansas, 1993.

Iannetti, A, Tacina, R. R., Cai, J., and Jeng, S.-M., "Multi-Swirler Aerodynamics: CFD Predictions," AIAA 2001-3575, 37th AIAA/ASME/SAE/ASEE Joint Propulsion Conference and Exhibit, Salt Palace, Salt Lake City, UT, USA, July 8-11, 2001a.

Iannetti, A., Tacina, R., Jeng, S. -M., and Cai, J., "Toward Accurate Prediction of Turbulence, Three-Dimensional, Recirculation Flows with the NCC," AIAA-20010809, 39th AIAA Aerospace Sciences Meeting and Exhibit, Reno, NV, USA, January 8-11, $2001 b$.

Im, K.-S., Lai, M.-C., and Tacina, R. R., “A Parametric Spray Study of the Swirler/Venturi Injectors," AIAA Paper 98-3269, 1998.

Jeng, S.-M., Cai, J., and Tacina, R.R., "Multi-Swirler Aerodynamics: Experimental Measurements," AIAA 2001-3574, 37th AIAA/ASME/SAE/ASEE Joint Propulsion Conference and Exhibit, Salt Palace, Salt Lake City, UT, USA, July 8-11, 2001.

Koff, B. L., “Aircraft Gas Turbine Emissions Challenge,” Journal of Engineering for Gas Turbines and Power, Vol. 116, No. 3, pp. 474-477, 1994.

Krollrack, R. and Aceto, L.D., "Nitric Oxide Formation in Gas Turbine Combustors," AIAA Journal, Vol. 11, No. 5, pp. 664-669, 1973. 
Kumakura, M. H., Sasaki, M., and Suzuki, D., "Low NOx Combustor for Automotive Ceramic Gas Turbine-Reliability Assurance," Journal of Engineering for Gas Turbines and Power, Vol. 115, No. 3, pp. 547-553, 1993.

Kundu, K.P., Penko, P.F., and Yang, S.L., "Reduced Reaction Mechanisms for Numerical Calculations in Combustion of Hydrocarbon Fuels," AIAA 98-0803, 36th AIAA Aerospace Sciences Meeting and Exhibit, Reno, Nevada, USA, January 12-15, 1998.

Laufer, J., "The Structure of Turbulence in Fully Developed Pipe Flow," NACA 1174, 1952.

Launder, B. E. and Sharma, B. I., "Application of the Energy-Dissipation Model of Turbulence to the Calculation of Flow Near a Spinning Disc," Letters in Heat and Mass Transfer, Vol. 1, pp. 131-138, 1974.

Launder, B. E. and Spalding, D. B., "Mathematical Models of Turbulence," Academic Press, New York, 1972.

Launder, B. E. and Spalding, D. B., “The Numerical Computation of Turbulent Flows," Computer Methods in Applied Mechanics and Engineering, Vol. 3, pp. 269-289, 1974.

Launder, B. E., "Second-moment Closure and Its Use in Modelling Turbulent Industrial Flows," International Journal for Numerical Methods in Fluids, Vol. 9, pp. 963985, 1989.

Launder, B. E., Reece, G. J., and Rodi, W., "Progress in the Development of a Re-stress Turbulence Closure,’ Journal of Fluid Mechanics, Vol. 68, pp. 537-566, 1975. 
Lefebvre, Arthur H., "Gas Turbine Combustion," Hemisphere Publishing Corporation, USA, 1983.

Leonard, G. and Stegmaier, J., "Development of an Aeroderivative Gas Turbine Dry Low Emissions Combustion System,” J. of Eng. for Gas Turbines and Power, Vol. 116, No. 3, pp. 542-546, 1994.

Lien, F. S. and Leschziner, M. A., "Assessment of Turbulece-Transport Models Including Non-linear RNG Eddy-Viscosity Formulation and Second-Moment Closure for Flow Over a Backward-Facing Step,” Computers and Fluids, Vol. 23, pp. 9831004, 1994.

Lin, C. A. and Lu, C. M., "Modeling Three-Dimensional Gas-Turbine Combustor Model Flow using a Second Moment Closure,’ AIAA Journal, Vol. 32, No. 7, pp. 1416$1422,1994$.

Liu, N.-S. and Quealy, A, "NCC - a Multi-Disciplinary Design/Analysis Tool for Combustion Systems," NASA 1998 Computational Aerosciences Workshop, August 1998.

Lumley, J. L., “Computational Modeling of Turbulent Flows,” Adv. Appl. Mech., Vol. 18, pp. 123-176, 1978.

Lumley, J. L., "Pressure-Strain Correlation,” Physics of Fluids, Vol. 18, No. 6, pp. 750$751,1975$.

Ma, Z., Wang, D., Jeng, S.-M., and Benjamin, M., "Flow Structures of a Radial-Inlet Swirler,' International Gas Turbine \& Aeroengine Congress \& Exhibition, Munich, Germany, May 8-11, 2000. 
Mansour, N. N., Kim, J., and Moin, P., "Reynolds-Stress and Dissipation-Rate Budgets in a Turbulent Channel Flow,” J. Fluid Mechanics, Vol. 194, pp. 15-44, 1988.

McGuirk, J. J. and Palma, J. M. L. M., “The Flow Inside a Model Gas Turbine Combustor: Calculations," J. of Eng. for Gas Turbines and Power, Vol. 115, No. 3, pp. 594-602, 1993.

Micklow, G. J., Roychoudhury, S., Nguyen, H. L., and Cline, M. C., "Emissions Reduction by Varying the Swirler Air Flow Split in Advanced Gas Turbine Combustors,” J. of Eng. for Gas Turbines and Power, Vol. 115, No. 3, pp 563-569, 1993.

Miller, J.A. and Bowman, C.T., "Mechanism and Modeling of Nitrogen Chemistry in Combustion," Progress in Energy and Combustion Science, Vol. 15, pp. 287-338, 1989.

O’Rourke, P. J. and Amsden, A. A., "Implementation of a Conjugate Residual Iteration in the KIVA Computer Program,” Los Alamos National Laboratory, LA-10849-MS, 1986.

Osher, S. and Chakravarthy, S., "Very High Order Accurate TVD Schemes," the IMA Volumes in Mathematics and its Applications, Vol. 2, Springer-Verlag, 1986, pp. 229-274.

Patankar, S.V., "Numerical Heat Transfer and Fluid Flow," Hemisphere Publishing Corporation, ISBN 0-07-048740-5, Washington D.C., 1980.

Penko, P.F., Kundu, K.P., and VanOverbeke, T.J., "A Practical Kinetic Mechanism for Computing Combustion in Gas Turbine Engines," AIAA 99-2218, 1999.

Pope, S. B., “Turbulent Flows,” Cambridge University Press, Cambridge, U. K., 2000. 
Pracht, W. E., "Calculating Three-Dimensional Fluid Flows at All Speeds With an Eulerian-Lagrangian Computing Mesh," Journal of Computational Physics, Vol. 17, pp. 132-159, 1975.

Quealy, A., Ryder, R., Norris, A., and Liu, N.-S., "National Combustion Code: Parallel Implementation and Performance," NASA TM-2000-209801, 2000.

Relation, H. L, Battaglioli, J. L., and Ng, W. F., "Numerical Simulations of Nonreacting Flows for Industrial Gas Turbine Combustor Geometries," Journal of Engineering for Gas Turbines and Power, Vol. 120, No. 1, pp. 460-467, 1998.

Reynolds, W. C., "Modeling of Fluid Motions in Engines - An Introductory Overview," in Combustion Modeling in Reciprocating Engines, Ed. J. N. Mattavi and C. A. Amann Plenum Press, New York, 1980.

Rhode, D. L., Lilley, D. G., and McLaughlin, D. K., "Mean Flowfields in Axisymmetric Combustor Geometries with Swirl," AIAA Journal, Vol. 21, No. 4, pp. 593-600, 1983.

Richardson, L. F., "Weather Prediction by Numerical Process," Cambridge University Press, Cambridge, U. K., 1922.

Rizk, N. K. and Mongia, H. C., "Three Dimensional Gas Turbine Combustor Emissions Modeling," Journal of Engineering for Gas Turbines and Power, Vol. 115, No. 3, pp. 603-611, 1993.

Roache, P. J., “Computational Fluid Dynamics,” Hermosa Publishers, Albuquerque, New Mexico, 1982.

Roe, P. L., "Generalized Formulation of TVD Lax-Wendroff Schemes," ICASE Report No. 84-53, 1984. 
Semerjian, H. and Vranos, A., "NOx Formation in Premixed Turbulent Flames," Sixteenth Symposium (International) of Combustion, The Combustion Institute, pp. 169$179,1976$.

Shih, T.-H., Chen, K.-H., and Liu, N.-S., “A Non-Linear $k$-epsilon Model for Turbulent Shear Flows," AIAA Paper 98-3983, 1998a.

Shih, T.-H., Chen, K.-H., Liu, N.-S., and Lumley, J. L., "Modeling of Turbulent Swirling Flows," NASA-TM - 113112, 1998 b.

Shih, T.-H. and Lumley, J. L., "Remarks on Turbulent Constitutive Relations," Mathematical and Computational Modeling, Vol. 18, pp. 1377-1387, 1993.

Shir, C. C., "A Preliminary Numerical Study of Atmospheric Turbulent Flows in the Idealized Planetary Boundary Layer," J. Atmos. Sci., Vol. 30, pp. 1327-1339, 1973.

Siow, Y. K., "A CFD Investigation of Cutting Fluid Mist Formation via Atomization," Ph.D Dissertation, Michigan Technological University, USA, 1999

Siow, Y. K., Yang, S. L., and Sutherland, J. W., "Study of Cutting Fluid Atomization Using Computational Fluid Dynamics," International Journal for Manufacturing Science and Production, Vol. 2, No. 2, pp. 144-152, 2000.

Sloan, D. G., Smith, P. J., and Smooth, L. D., "Modeling of Swirl in Turbulent Flow Systems," Progress in Energy and Combustion Science, Vol. 12, pp. 103-250, 1986.

Speziale, C. G., Sarkar, S., and Gatski, T. B., "Modelling the Pressure-Strain Correlation of Turbulence: An Invariant Dynamical System Approach,” J. Fluid Mechanics, Vol. 227, pp. 245-272, 1991. 
Stubbs, R.M. and Liu, N.-S., "Preview of the National Combustion Code," AIAA 973314, 33rd AIAA/ASME/SAE/ASEE Joint Propulsion Conference and Exhibit, July 6-9, Seattle, WA, USA, 1997.

Tacina, R. R., “Low NOx Potential of Gas Turbine Engines,” AIAA Paper 90-0550, 28th Aerospace Sciences Meeting and Exhibit, Reno, NV, 1990.

Talpallikar, M. V., Smith, C. E., Lai, M. C., and Holdeman, J. D., “CFD Analysis of Jet Mixing in Low NOx Flametube Combustors," Journal of Engineering for Gas Turbines and Power, Vol. 114, No. 2, pp. 416-424, 1992.

Teo, C. Y., Siow, Y. K., and Yang, S. L., "Flowfield Study of LDI Combustor with Discrete- Jet Swirler Using Re-Stress Model," AIAA Paper 2001-3424, 37th AIAA/ASME/SAE/ ASEE Joint Propulsion Conference and Exhibit, Salt Lake City, UT, USA, July 8-11, 2001.

Tsao, J. M. and Lin, C. A., "Reynolds Stress Modeling of Jet and Swirl Interaction Inside a Gas Turbine Combustor,” International Journal for Numerical Methods in Fluids, Vol. 29, No. 4, pp. 451- 464, 1999.

Wilcox, D. C., “Turbulence Modeling for CFD,” La Canada, CA, DCW Industries, 1993. Wilson, K. G., "Renormalization Group and Strong Interactions," Physical Review D, Vol. 3, pp. 1818-1846, 1971.

Yakhot, V. and Orszag, S. A., "Renormalization Group Analysis of Turbulence. I. Basic Theory," Journal of Scientific Computing, Vol. 1, pp. 3-51, 1986.

Yang, S. L., Chang, Y. L., Arici, O., "Incompressible Navier-Stokes Computation of the NREL Airfoils Using a Symmetric Total Variational Diminishing Scheme," Transactions of the ASME, Vol. 116, pp. 174-182, November 1994. 
Yang, S. L., Chen, R., Cline, M. C., and Nguyen, H. L., "Numerical Simulation of a LowEmission Gas Turbine Combustor Using KIVA-II," International Journal for Numerical Methods in Fluids, Vol. 15, pp. 865-881, 1992.

Yang, S. L., Peschke, B. D., and Hanjalic, K., "Second-Moment Closure Model for IC Engine Flow Simulation Using Kiva Code," Journal of Engineering for Gas Turbines and Power, Vol. 122, pp. 355-363, 2000.

Yang, S. L., Siow, Y. K., Peschke, B. D., and Tacina, R. R., "Numerical Study of Nonreacting Gas Turbine Combustor Swirl Flow Using Reynolds Stress Model,” to appear in Journal of Engineering for Gas Turbines and Power, 2003a.

Yang, S. L., Teo, C. Y., Siow, Y. K., Tacina, R. R., and Penko, P. F., "Numerical Study of LDI Combustor with Discrete-Jet Swirlers Using Re-Stress Model," to appear in Journal of Engineering for Gas Turbines and Power, $2003 \mathrm{~b}$.

Yee, H. C., "Upwind and Symmetric Shock-Capturing Schemes," NASA TM-89464, 1987. 
Appendix A

In-House Coding of Reynolds-Stress Turbulence Model in KIVA 
This appendix describes the major coding modifications to the original KIVA-3V (Release 2) hydrocode. Twenty subroutines and approximately 7,000 lines have been added to KIVA. An addition of 130 major arrays (with vector length equal to at least the total number of vertices) have been included in the common blocks, in the file include.i. These added arrays are equivalenced wherever possible, to minimize the required memory. For a typical run with 3-D geometry and reasonably fine grid, the added arrays result in (approx.) 20-30\% higher run-time memory with the RSTM than with the standard $k-\varepsilon$ model

The input file itape 5 is also affected as a result of the incorporation of RSTM. The existing parameter that now takes on new values is:

$$
\begin{array}{ll}
\text { turbsw: } & =\text { no turbulence model } \\
1 & =\text { standard } k-\varepsilon \text { model } \\
2 & =\text { RNG } k-\varepsilon \text { model } \\
11 & =\text { SSG RSTM } \\
12 & =\text { LRR RSTM }
\end{array}
$$

The added RSTM-related parameter is:

$$
\begin{aligned}
& \text { tke1st: } \quad 1=\text { call subroutine } r \text { smget } \\
& 0=\text { otherwise } \\
& \text { where rsmget initializes Reynolds-stresses using pre-calculated k-e }
\end{aligned}
$$

Several non-RSTM-related parameters have also been included in the input file. These parameters provide extra features described below: 
idilate: a switch to zero all dilatation terms in the turbulence equations, suitable for stationary grids - i.e., grids without moving boundaries

ny : $\quad$ number of cells in the $\mathrm{j}$-direction, for identifying $2-\mathrm{D}$ or 3 -D planar grids

dyperd: thickness of cells in the j-direction for 2-D or 3-D planar grids, used when updating periodic boundaries

tapein: a switch to use user-specified inflow data (e.g., fully-developed pipe flow)

from a file named “itape.inflow," bypassing KIVA's original inlet boundary data read

tapout: a switch to write outflow data to a file called "otape.outflow," to be renamed "itape. inflow" when used in conjunction with tapein

subcin: a switch to call subroutine bccbin, a modified version of KIVA's bcccin; used in conjunction with tapein

subcout: similar to subcin, except it is for outflow boundary where pressure is extrapolated

tbcycle: the cycle interval at which to call subroutine period, to set inflow conditions equal to outflow, for periodic in/out boundaries (e.g., study of fully developed pipe flow)

bdrytab: a switch to call subroutine bctable where all boundary data are written to a file named "bctable.dat," for debugging purposes

wallpts: a switch to call subroutine wallchk where turbulence quantities near solid walls are written to the file "wallchk . dat"

cnvrgck: a switch to call subroutine convrg to check for convergence in steadystate simulations 
Approximately 7,000 lines and 20 subroutines have been added as a results of the inclusion of Reynolds-stress turbulence model.

The following flow-chart illustrates the progress of the Reynolds-stress computation. All participating subroutines are shown, including the routines called by these subroutines. The RSTM-related subroutine names are in bold font. A short description, in italic font, of the purpose of each subroutine follows immediately below the subroutine name. The vertical arrows represent the advancement in time for a given cycle. The horizontal arrows represent the call sequence. 


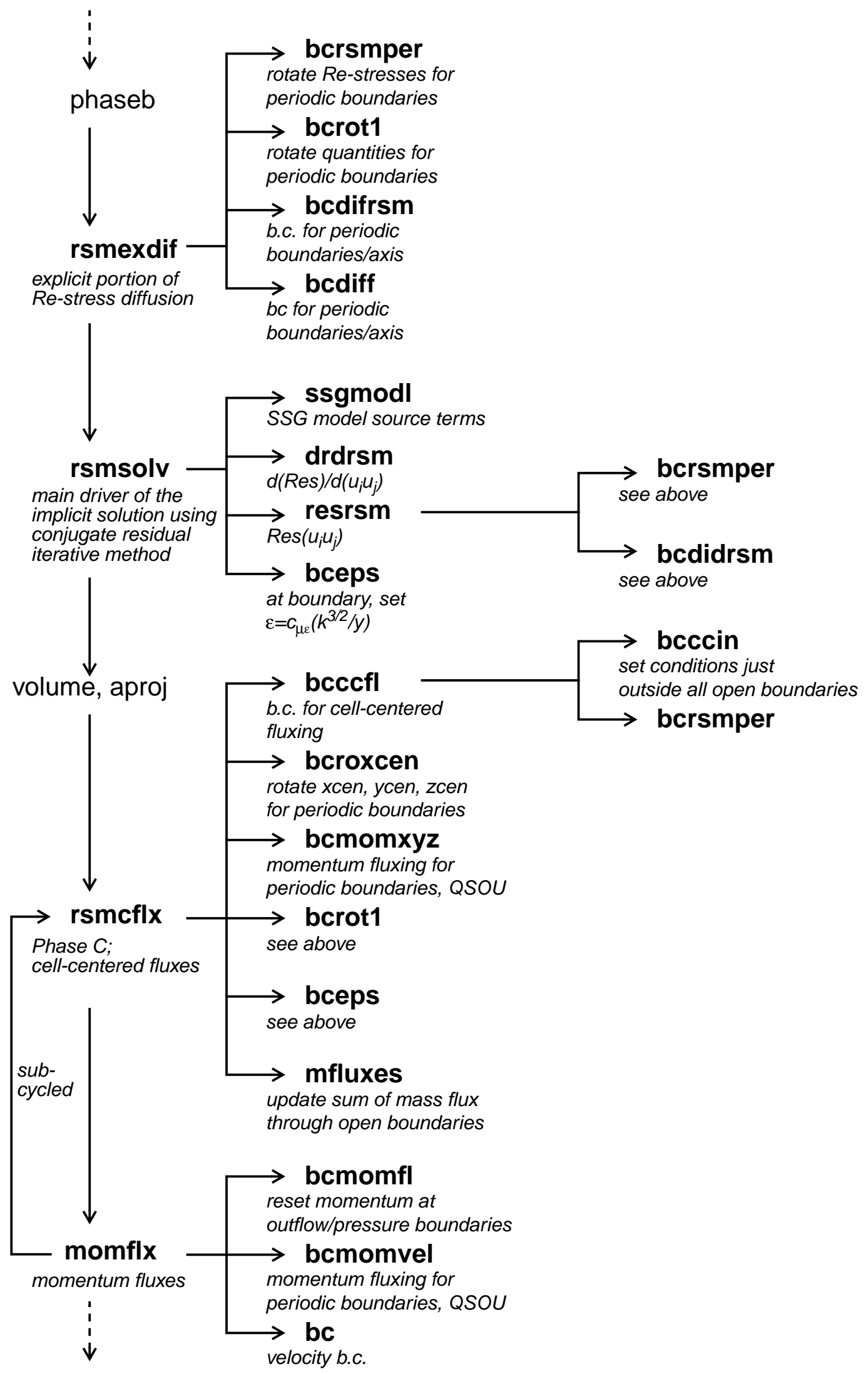




\section{Appendix B}

16-Species, 23-Step Mechanism for Jet-A Fuel 


\begin{tabular}{|c|c|c|c|c|}
\hline & Chemical Equation & $\mathrm{A}^{\mathrm{i}}$ & $\beta$ & $\mathrm{E}^{\mathrm{ii}}$ \\
\hline \multicolumn{5}{|c|}{ Global Reactions: } \\
\hline 1. & $\begin{array}{l}\left\{N_{2}\right\}+C_{12} H_{23} \rightarrow 12 C H+11 H+\left\{N_{2}\right\} \\
\mathrm{a}=0.8 \quad \mathrm{~b}=0.8\end{array}$ & $1.00 \mathrm{E}+11$ & 0.0 & 30000 \\
\hline $2 \mathrm{f}$. & $\begin{array}{l}\{\mathrm{CH}\}+\mathrm{H}_{2}+\mathrm{N}_{2} \rightarrow 2 \mathrm{NH}+\{\mathrm{CH}\} \\
\mathrm{a}=1.0 \quad \mathrm{~b}=0.1 \quad \mathrm{c}=1.0\end{array}$ & $1.00 \mathrm{E}+16$ & 0.0 & 78000 \\
\hline $2 \mathrm{~b}$. & $\begin{array}{l}\{\mathrm{CH}\}+2 \mathrm{NH} \rightarrow \mathrm{N}_{2}+\mathrm{H}_{2}+\{\mathrm{CH}\} \\
\mathrm{a}=1.0 \quad \mathrm{~b}=2.0\end{array}$ & $1.95 \mathrm{E}+15$ & 0.0 & 0 \\
\hline $3 \mathrm{f}$. & $\begin{array}{l}\{O\}+N_{2}+H_{2} \rightarrow 2 N O+H+\{O\} \\
a=0.1 \quad b=0.5 \quad c=1.0\end{array}$ & $1.25 \mathrm{E}+08$ & 0.5 & 45898 \\
\hline $3 b$. & $\begin{array}{l}2 \mathrm{NO}+\mathrm{H} \rightarrow \mathrm{N}_{2}+\mathrm{HO}_{2} \\
\mathrm{a}=1.1 \quad \mathrm{~b}=1.0\end{array}$ & $2.50 \mathrm{E}+10$ & 0.0 & 8000 \\
\hline $4 f$. & $\begin{array}{l}N_{2}+O \rightarrow N+N O \\
\mathrm{a}=0.5 \mathrm{~b}=1.0\end{array}$ & $3.75 \mathrm{E}+10$ & 0.27 & 75000 \\
\hline $4 \mathrm{~b}$. & $\mathrm{N}+\mathrm{NO} \rightarrow \mathrm{N}_{2}+\mathrm{O}$ & $3.00 \mathrm{E}+12$ & 0.2 & 0 \\
\hline \multicolumn{5}{|c|}{ Molecular Reactions: } \\
\hline 5. & $\left\{\mathrm{~N}_{2}\right\}+2 \mathrm{CH} \leftrightarrow \mathrm{C}_{2} \mathrm{H}_{2}+\left\{\mathrm{N}_{2}\right\}$ & $1.00 \mathrm{E}+15$ & 0.0 & 0 \\
\hline 6. & $\mathrm{C}_{2} \mathrm{H}_{2}+\mathrm{O}_{2} \leftrightarrow 2 \mathrm{CO}+\mathrm{H}_{2}$ & $3.00 \mathrm{E}+15$ & 0.0 & 19000 \\
\hline 7. & $\mathrm{H}_{2}+\mathrm{OH} \leftrightarrow \mathrm{H}_{2} \mathrm{O}+\mathrm{H}$ & $5.00 \mathrm{E}+06$ & 1.0 & 3626 \\
\hline 8. & $\mathrm{H}_{2}+\mathrm{O} \leftrightarrow \mathrm{H}+\mathrm{OH}$ & $2.50 \mathrm{E}+15$ & 0.0 & 6000 \\
\hline 9. & $\mathrm{H}+\mathrm{O}_{2} \leftrightarrow \mathrm{O}+\mathrm{OH}$ & $4.00 \mathrm{E}+14$ & 0.0 & 18000 \\
\hline $10 \mathrm{f}$ & $\mathrm{N}_{2}+\mathrm{O}_{2} \leftrightarrow 2 \mathrm{O}+\mathrm{N}_{2}$ & $1.00 \mathrm{E}+18$ & 0.0 & 122240 \\
\hline $10 \mathrm{~b}$. & $2 \mathrm{O}+\mathrm{H}_{2} \leftrightarrow \mathrm{H}_{2}+\mathrm{O}_{2}$ & $1.00 \mathrm{E}+18$ & 0.0 & 0 \\
\hline 11. & $\mathrm{CO}+\mathrm{OH} \leftrightarrow \mathrm{CO}_{2}+\mathrm{H}$ & $1.51 \mathrm{E}+07$ & 1.28 & -758 \\
\hline 12. & $\mathrm{H}_{2}+2 \mathrm{H} \leftrightarrow 2 \mathrm{H}_{2}$ & $4.00 \mathrm{E}+20$ & 0.6 & 0 \\
\hline 13. & $\mathrm{CH}+\mathrm{O} \leftrightarrow \mathrm{CO}+\mathrm{H}$ & $1.00 \mathrm{E}+12$ & 0.5 & 0 \\
\hline 14. & $\mathrm{H}+\mathrm{O}_{2} \leftrightarrow \mathrm{HO}_{2}$ & $1.00 \mathrm{E}+15$ & -1.2 & 0 \\
\hline 15. & $\mathrm{CO}+\mathrm{HO}_{2} \leftrightarrow \mathrm{CO}_{2}+\mathrm{OH}$ & $1.8 \mathrm{E}+12$ & 0.0 & 22932 \\
\hline 16. & $\mathrm{O}+\mathrm{HO}_{2} \leftrightarrow \mathrm{OH}+\mathrm{O}_{2}$ & $1.5 \mathrm{E}+13$ & 0.0 & 0 \\
\hline 17. & $\mathrm{H}+\mathrm{HO}_{2} \leftrightarrow \mathrm{H}_{2}+\mathrm{O}_{2}$ & $1.5 \mathrm{E}+14$ & 0.0 & 0 \\
\hline 18. & $N_{2}+2 N \leftrightarrow 2 N_{2}$ & $1.00 \mathrm{E}+15$ & 0.0 & 0 \\
\hline 19. & $\mathrm{~N}+\mathrm{O}_{2} \leftrightarrow \mathrm{NO}+\mathrm{O}$ & $6.30 \mathrm{E}+09$ & 1.0 & 6300 \\
\hline 20. & $N+O H \leftrightarrow N O+H$ & $3.00 \mathrm{E}+13$ & 0.0 & 0 \\
\hline 21. & $N H+O \leftrightarrow N O+H$ & $1.50 \mathrm{E}+13$ & 0.0 & 0 \\
\hline 22. & $\mathrm{NH}+\mathrm{NO} \leftrightarrow \mathrm{N}_{2}+\mathrm{OH}$ & $2.00 \mathrm{E}+15$ & -0.8 & 0 \\
\hline & $\mathrm{CH}+\mathrm{NO} \leftrightarrow \mathrm{NH}+\mathrm{CO}$ & $1.00 \mathrm{E}+11$ & 0.0 & 0 \\
\hline
\end{tabular}

i. A is for reaction pressure of $5 \mathrm{~atm}$. Units are $\mathrm{cm}-\mathrm{sec}-$ mole-cal-Kelvins

ii. $\mathrm{E}$ is in $\mathrm{cal} / \mathrm{mole}$

Definition of Terms:

$k_{f}=A T^{\beta} e^{-E /(R T)}[X]^{a}[Y]^{b}[Z]^{c}$

Unless otherwise specified, $a, b$, and $c$ are the forward reaction, stoichiometric coefficients:

$a X+b Y+c Z \rightarrow$ products

The letters $f$ and $b$ refer to specified forward and backward reactions, respectively. Backward reactions are otherwise calculated by the code if not specified. 


\section{The file mechanism includes the following lines:}

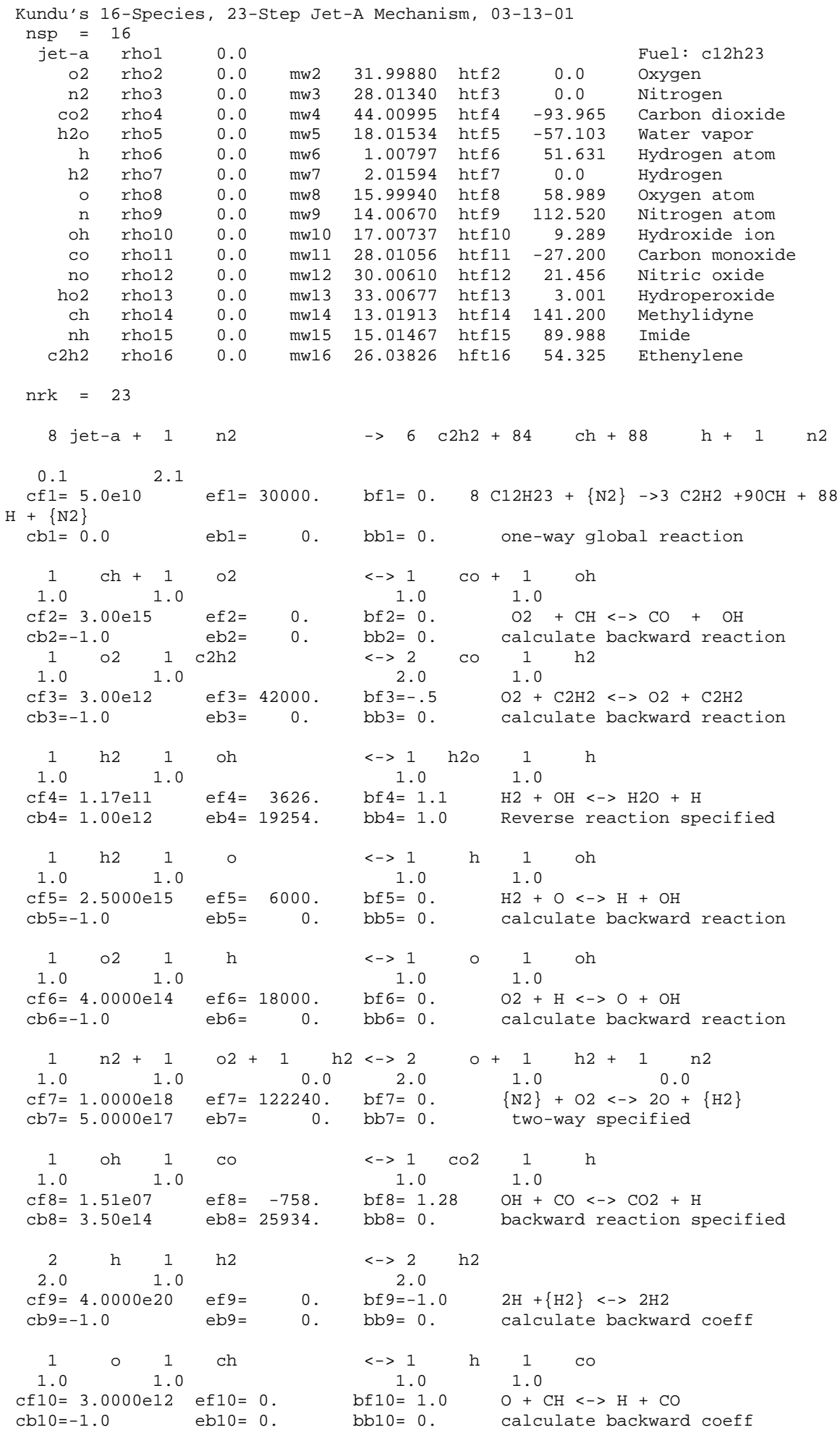




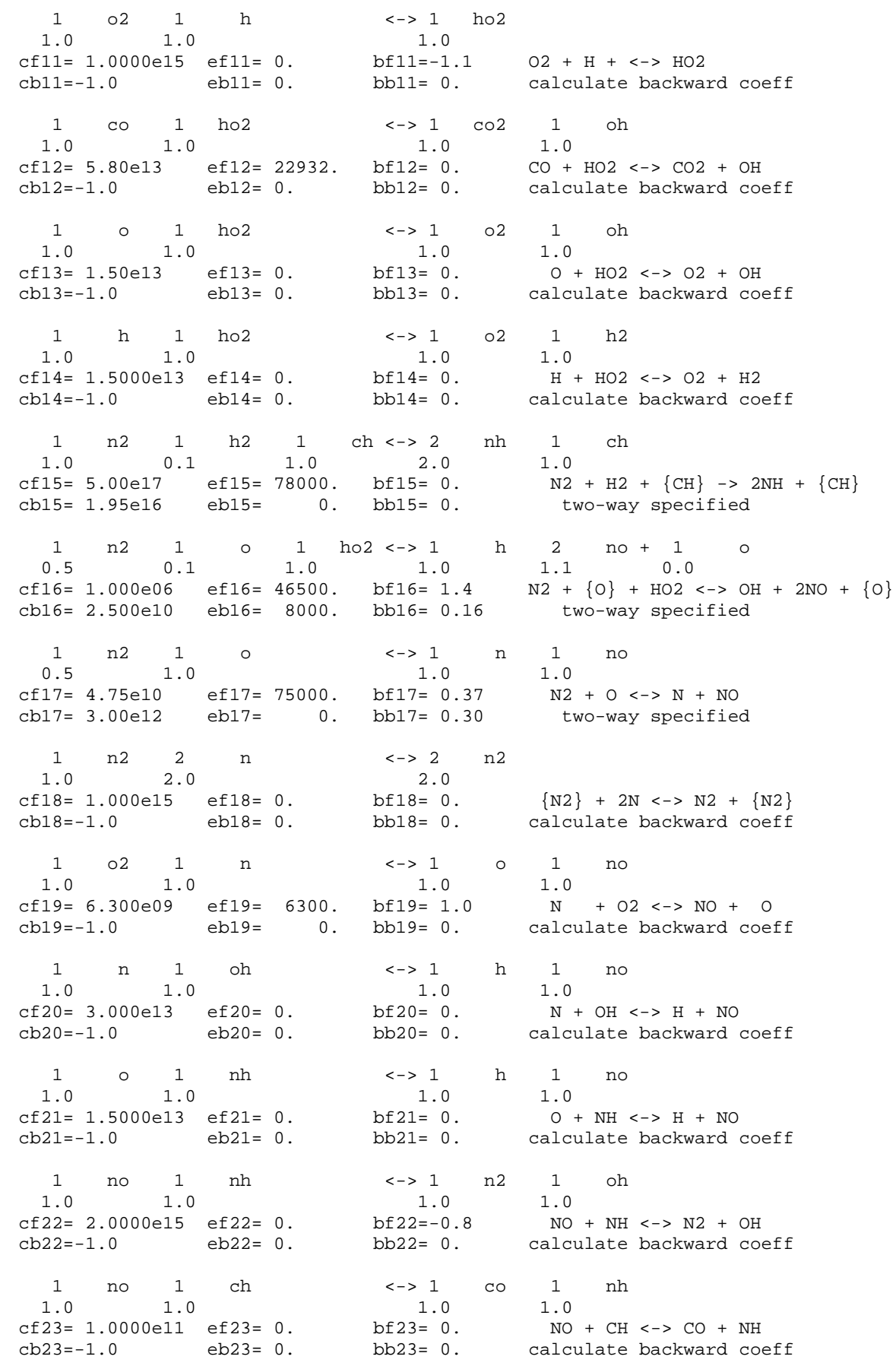


Appendix C

Rezone Phase (Phase C) Solution Procedure in KIVA 


\section{C.1. General Description}

After the Lagrangian phases (Phases A and B) in KIVA, all quantities will have been updated with newly computed values due to the effects of diffusion, pressurevelocity coupling, and source terms including chemistry and spray. The remaining convective transport is now taken care of in Phase C, where the flow field is frozen in time while each grid point in the computational mesh is relocated to its new coordinates. The convective fluxes due to the grid motion relative to the flow field are calculated, using some differencing technique. Available in KIVA are the partial donor cell (PDC) and quasi-second-order upwind (QSOU) schemes, in additional to the newly implemented total-variation diminishing (TVD) scheme.

Phase $\mathrm{C}$ is accomplish in a subcycled, explicit manner using a time step $\Delta t_{c} . \Delta t_{c}$ must satisfy the Courant-Friedrichs-Levy (CFL) stability condition, i.e., $\mathrm{CFL}<1$, since both PDC and QSOU schemes in KIVA are both forward-time upwind-space finitedifference methods.

Accordingly, the number of subcycle $(N S)$ is

$$
N S=\frac{\Delta t}{\Delta t_{c}}
$$

The rezone calculation takes only about $10 \%$ of the time taken by the Lagrangian phase calculation.

Monotonicity is an important property for any finite-difference approximation method. It has been shown that all monotone schemes converge to solutions that are physically correct [Harten et al., 1976; Crandall and Majda, 1980], particularly near discontinuities. Strongly-monotone schemes also do not suffer from spurious oscillations 
as found in other methods such as central-difference and some higher-order schemes [Roache, 1982].

The two convection schemes available in KIVA, quasi-second-order upwind (QSOU) and partial donor cell (PDC), carry different monotone properties and accuracy. QSOU is strongly monotone, and second-order accurate in space only when $\Delta f / \Delta x$ is a constant everywhere in the domain, where $f$ is the quantity to be convected, and $\Delta x$ is the grid spacing. It otherwise reduces to partial donor cell or first-order upwind (pure donor cell), depending on how $f$ varies in space. First-order upwind scheme is strongly monotone but highly diffusive.

The partial donor cell method, on the other hand, is not monotone, although it is less diffusive than the first-order upwind scheme. The user can control the amount of upwinding (pure, interpolated, or central) by adjusting two parameters in the PDC formulation. Although it is less accurate than the QSOU method, the PDC scheme is computationally inexpensive by comparison.

Features of the TVD scheme have been described in Chapter 6 of this dissertation.

\section{C.2. Phase C Numerical Methods}

The numerical methods employed in Phase $\mathrm{C}$ is described next.

\section{C.2.1. Cell-Centered Quantities}

The transport of cell-centered quantities are computed by using a volume $\delta V_{a}$ that is swept out by regular cell face $a$ in each convective subcycle, as it moves from $\vec{x}_{i j k}^{B}$ to 
$\vec{x}_{i j k}^{n+1}$. Figure 6.1 shows such deformation of a cell in two dimensions. The solid circles represent the points $\left(x_{i j}^{B}, y_{i j}^{B}\right)$ and the open circles, $\left(x_{i j}^{n+1}, y_{i j}^{n+1}\right)$. Positive (negative) volume changes are indicated by positive (negative) sloped shadings. The volume changes cancel in doubly shaded areas.

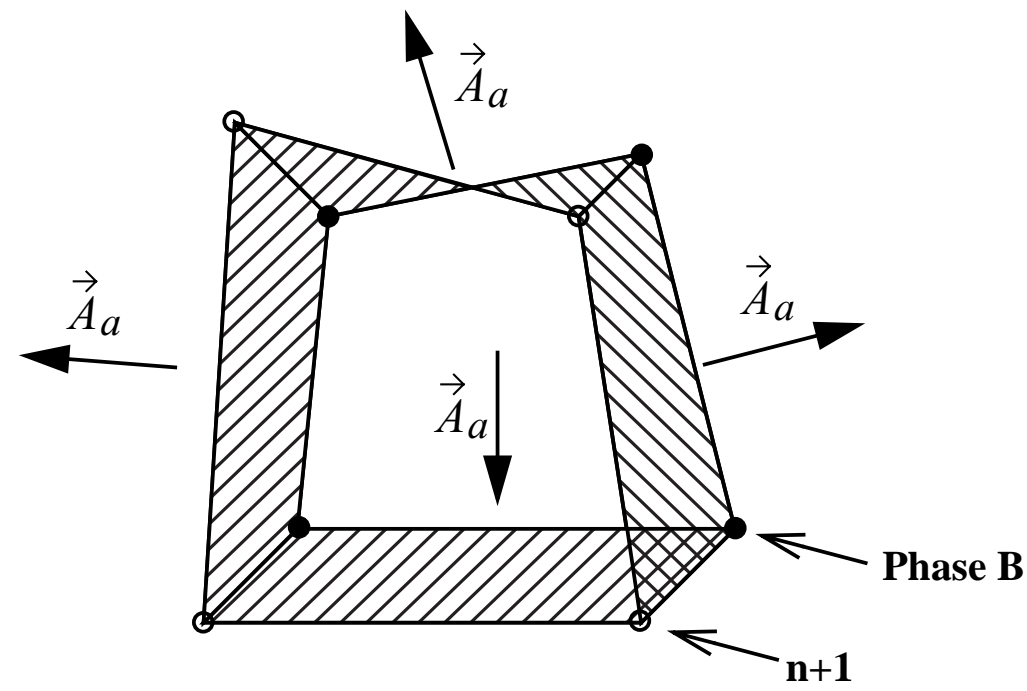

Figure C.1. Illustration of cell volume changes in Phase C

(1) Calculation of $\delta V_{a}$

$\delta V_{a}$, the volume swept out by a cell face in each subcycle, is evaluated in terms of the cell-face velocity and the old-time (n) and new-time $(n+1)$ grid positions, i.e.,

$$
\delta V_{a}=\delta V_{a}^{G} \frac{\Delta t_{c}}{\Delta t}-(u A)_{a}^{B} \Delta t_{c}
$$

where

$$
\frac{\Delta t_{c}}{\Delta t}=\frac{1}{N S}
$$


In Eq. (C.1), $\delta V_{a}^{G}$ is the volume swept out by cell face $a$ when the four vertices defining the face move from their old-time positions, $\vec{x}_{i j k}$, to their new-time coordinates, $\vec{x}_{i j k}^{n+1}$.

From the volume-change equation, i.e., $V_{i j k}^{B}=V_{i j k}^{n}+\Delta t \sum_{a}(u A)_{a}^{B}$, it can be shown that $\delta V_{a}$ satisfies

$$
V_{i j k}^{n+1}=V_{i j k}^{B}+N S \sum_{a} \delta V_{a}
$$

i.e.,

$$
\sum_{a} \delta V_{a}=\frac{V_{i j k}^{n+1}-V_{i j k}^{B}}{N S}
$$

which is the total volume change for each subcycle in Phase C.

(2) Calculation of species densities $\left(\rho_{m}\right)_{i j k}^{v}$

After the change of volume, $\delta V_{a}$, has been calculated, other cell-centered thermodynamic quantities can be evaluated in Phase C. Species density, after $v$ convective subcycles, is calculated according to:

$$
\left(\rho_{m}\right)_{i j k}^{v} V_{i j k}^{v}=\left(\rho_{m}\right)_{i j k}^{v-1} V_{i j k}^{v-1}+\sum_{a}\left(\rho_{m}\right)_{a}^{v-1} \delta V_{a}
$$

where

$$
\left(\rho_{m}\right)_{i j k}^{0}=\left(\rho_{m}\right)_{i j k}^{B}
$$

The cell volume at each subcycle is

$$
V_{i j k}^{v}=\left[v V_{i j k}^{n+1}+(N S-v) V_{i j k}^{B}\right] / N S
$$

In Eq. (C.5), $\left(\rho_{m}\right)_{a}^{v}$ can be obtained either by the total-variation diminishing (TVD), quasi-second-order upwind (QSOU) or partial donor cell (PDC) schemes. 
The total density after $v$ convective subcycles is thus given by

$$
\rho_{i j k}^{v}=\sum_{m}\left(\rho_{m}\right)_{i j k}^{v}, m=1,2, \ldots, n s p
$$

where $n s p$ is the total number of species.

The cell mass after $v$ convective subcycles can now be found from Eq. (C.7) and Eq. (C.8):

$$
M_{i j k}^{v}=\rho_{i j k}^{v} V_{i j k}^{v}
$$

(3) Calculation of specific internal energy $I_{i j k}^{v}$

Similar to species density, the specific internal energy is evaluated with the following relation:

$$
\rho_{i j k}^{v} V_{i j k}^{v} I_{i j k}^{v}=\rho_{i j k}^{v-1} V_{i j k}^{v-1} I_{i j k}^{v-1}+\sum_{a}(\rho I)_{a}^{v-1} \delta V_{a}
$$

where the term $(\rho I)_{a}$ is evaluated via either TVD, QSOU or PDC methods.

(4) The turbulence quantities $q_{i j k}^{v}$

All turbulence quantities, e.g., turbulent kinetic energy $k$, are cell-centered and therefore handled similarly as above:

$$
\rho_{i j k}^{v} V_{i j k}^{v} q_{i j k}^{v}=\rho_{i j k}^{v-1} V_{i j k}^{v-1} q_{i j k}^{v-1}+\sum_{a}(\rho q)_{a}^{v-1} \delta V_{a}
$$

where either TVD, QSOU or PDC can be used to calculate $(\rho q)_{a}$.

The turbulence quantities convected include the Reynolds-stresses, turbulent kinetic energy, and turbulence length scale. This length scale is related to turbulent kinetic energy and its dissipation rate, given by

$$
L=\frac{k^{3 / 2}}{\varepsilon}
$$


$L$ is used in convection because $\varepsilon$ generally has steeper gradients, and therefore higher numerical errors could arise when convecting $\varepsilon$.

(5) Other cell-centered quantities

Temperature is obtained by inverting

$$
I(T)=\sum_{m}\left(\frac{\rho_{m}}{\rho}\right) I_{m}(T)
$$

using values of internal energy $I$ and densities.

The pressure is given by the state relation:

$$
P_{i j k}=R_{0} T_{i j k} \sum_{m} \frac{\left(\rho_{m}\right)_{i j k}}{W_{m}}, m=1,2, \ldots, n s p
$$

where the summation is carried out for the total number of species (nsp). The quantity $R_{0}$ is the universal gas constant, and $W$ is the molecular weight of the species.

\section{C.2.2. Vertex Quantities (i.e., Momentum or Velocity $\vec{u}$ )}

Convective transport of momentum on subcycle $v$ is calculated in terms of the mass increments across momentum cell faces, which are related to the mass increments across regular cell faces.

(1) Mass increment across momentum cell face $a$

The mass increment for each convective subcycle for a given cell face is:

$$
\left(\delta M_{a}^{\prime}\right)^{v-1}=\frac{1}{8}\left(\rho_{o}^{v-1} \delta V_{o}-\rho_{i}^{v-1} \delta V_{i}\right)
$$

where the subscripts $o$ and $i$ refer to the regular cell faces on either side of the momentum cell face $a$, as shown by the schematic in Figure C.2 below. 


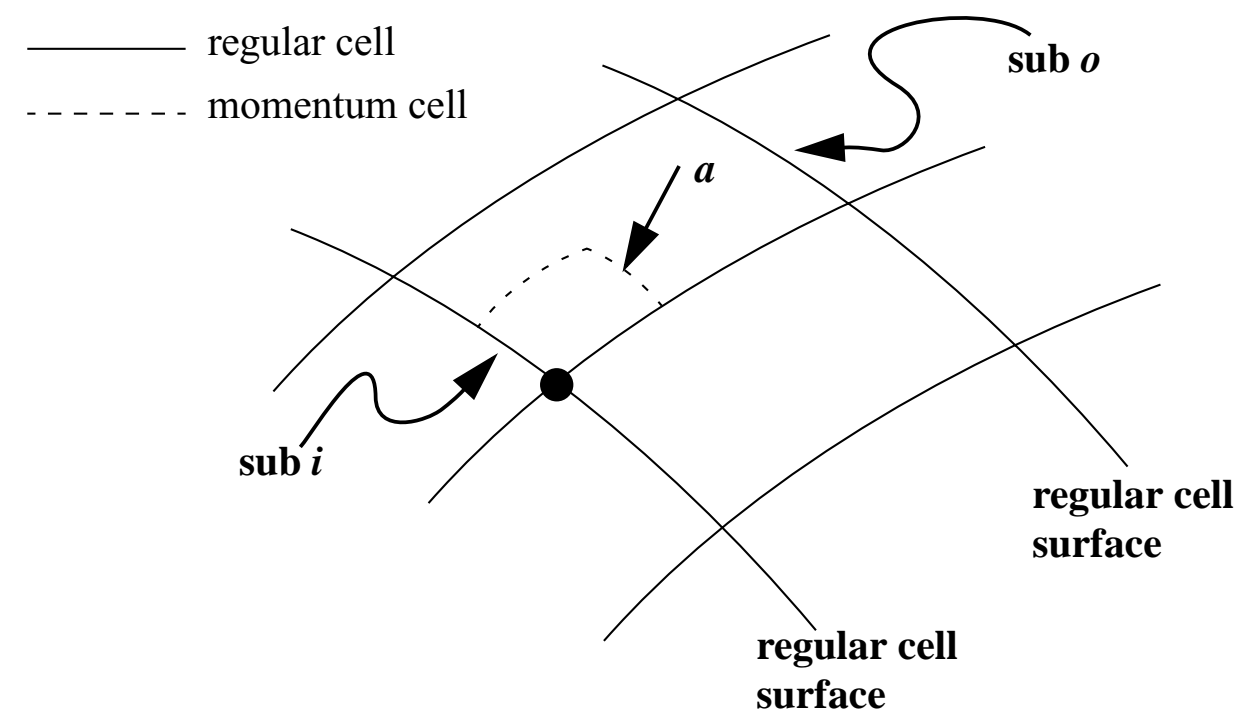

Figure C.2. Momentum and Regular Cell Faces

(2) Mass flux through a composite face $\beta$

A composite face, as illustrated by the 2-D schematic drawing in Figure C.3, comprises the four momentum cell faces $a$ intersecting a common regular cell edge $E$ (pointing into and outward from the paper).
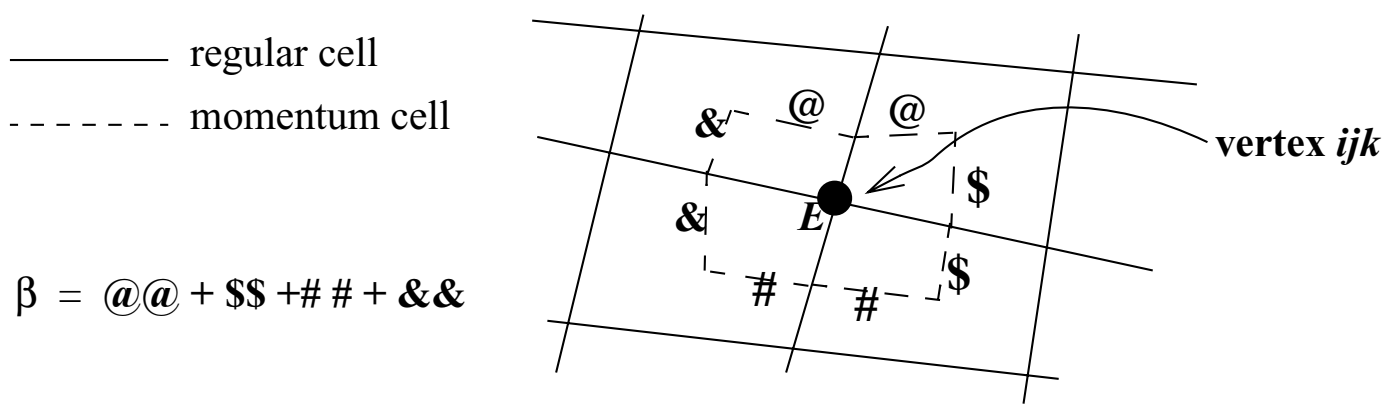

Figure C.3. Composite Faces 
For the 3-D case, there are a total of six composite faces for a momentum cell.

The mass increments, $\delta M^{\prime}{ }_{a}$, from (1) above, is then summed in groups (e.g., $\$ \$$ etc., c.f. Figure C.3) to obtain mass flux through a composite face $\beta$, i.e., $\delta M_{\beta}^{C}$.

(3) Momentum Flux

Having calculated $\delta M_{\beta}^{C}$, the momentum fluxes can now be determined:

$$
\left(M^{\prime}\right)_{i j k}^{v} \vec{u}_{i j k}^{v}=\left(M^{\prime}\right)_{i j k}^{v-1} \vec{u}_{i j k}^{v-1}+\sum_{\beta}\left(\delta M_{\beta}^{C}\right)^{v-1} \vec{u}_{\beta}^{v-1}
$$

where the velocity $\vec{u}_{\beta}$ is evaluated by TVD, QSOU or PDC schemes. 